Ulrich Derenthal

Geometry of universal torsors

Dissertation 



\title{
Geometry of universal torsors
}

\author{
Dissertation \\ zur Erlangung des Doktorgrades \\ der Mathematisch-Naturwissenschaftlichen Fakultäten \\ der Georg-August-Universität zu Göttingen
}

vorgelegt von

Ulrich Derenthal

aus

Brakel

Göttingen, 2006 
Referent: Prof. Dr. Yuri Tschinkel

Korreferent: Prof. Dr. Victor Batyrev

Tag der mündlichen Prüfung: 13. Oktober 2006 


\section{Preface}

Many thanks are due to my advisor, Prof. Dr. Yuri Tschinkel, for introducing me to the problems considered here and for his guidance.

I have benefitted from many discussions, especially with V. Batyrev, H.-C. Graf v. Bothmer, R. de la Bretèche, T. D. Browning, B. Hassett, J. Hausen, D. R. Heath-Brown, J. Heinloth, M. Joyce, E. Peyre, and P. Salberger. I am grateful for their suggestions and advice.

Parts of this work were done while visiting the University of Oxford (March 2004), Rice University (February 2005), the CRM at Université de Montréal (July 2005) during the special year on Analysis in Number Theory, the Courant Institute of Mathematical Sciences at New York University (December 2005), and the MSRI in Berkeley (March-May 2006) during the program Rational and Integral Points on Higher-Dimensional Varieties. I thank these institutions for their support and ideal working conditions.

Calculations leading to some of my results were carried out on computers of the Gauss-Labor (Universität Göttingen).

Financial support was provided by Studienstiftung des deutschen Volkes and DFG (Graduiertenkolleg Gruppen und Geometrie).

For proofreading, thanks go to Kristin Stroth and Robert Strich.

I am grateful to my parents Anne-Marie and Josef Derenthal and my sisters Birgitta and Kirstin for their support and encouragement. Finally, I thank Inga Kenter for the wonderful time during the last years.

Ulrich Derenthal 



\section{Contents}

Preface $\quad$ v

$\begin{array}{ll}\text { Introduction } & 1\end{array}$

Part 1. Universal torsors of Del Pezzo surfaces

$\begin{array}{ll}\text { Chapter 1. Del Pezzo surfaces } & 7\end{array}$

$\begin{array}{ll}\text { 1.1. Introduction } & 7\end{array}$

$\begin{array}{lll}1.2 . & \text { Blow-ups of smooth surfaces } & 8\end{array}$

1.3. Smooth Del Pezzo surfaces 11

\begin{tabular}{lll}
\hline 1.4. & Weyl groups and root systems & 12
\end{tabular}

$\begin{array}{lll}1.5 . & \text { Singular Del Pezzo surfaces } & 15\end{array}$

\begin{tabular}{lll}
\hline 1.6. & Classification of singular Del Pezzo surfaces & 17
\end{tabular}

1.7. Contracting $(-1)$-curves 20

1.8. Toric Del Pezzo surfaces 22

Chapter 2. Universal torsors and Cox rings 25

2.1. Introduction 25

2.2 . Universal torsors 25

$\begin{array}{lll}2.3 . & \text { Cox rings } & 27\end{array}$

$\begin{array}{lll}2.4 . & \text { Generators and relations } & 27\end{array}$

$\begin{array}{lll}\text { Chapter 3. } & \text { Cox rings of smooth Del Pezzo surfaces } & 31\end{array}$

3.1. Introduction 31

$3.2 . \quad$ Relations in the Cox ring 31

\begin{tabular}{ll|l}
3.3. & Degree 3 & 34
\end{tabular}

\begin{tabular}{lll}
\hline 3.4. & Degree 2 & 38
\end{tabular}

$\begin{array}{lll}3.5 . \quad \text { Degree } 1 & 40\end{array}$

Chapter 4. Universal torsors and homogeneous spaces 45

4.1. Introduction 45

\begin{tabular}{lll}
\hline 4.2. & Homogeneous spaces & 47
\end{tabular}

\begin{tabular}{lll}
\hline 4.3. & Rescalings & 50
\end{tabular}

\begin{tabular}{lll}
\hline 4.4. & Degree 3 & 53
\end{tabular}

\begin{tabular}{lll}
\hline 4.5. & Degree 2 & 55
\end{tabular}

Chapter 5. Universal torsors which are hypersurfaces 57

$\begin{array}{lll}5.1 . & \text { Introduction } & 57\end{array}$

$\begin{array}{lll}5.2 . & \text { Strategy of the proofs } & 58\end{array}$

$\begin{array}{lll}5.3 . & \text { Degree } \geq 6 & 61\end{array}$

\begin{tabular}{lll}
\hline 5.4. & Degree 5 & 63
\end{tabular}

$\begin{array}{lll}5.5 . \quad \text { Degree } 4 & 66\end{array}$ 
\begin{tabular}{lll}
\hline 5.6. & Degree 3 & 73
\end{tabular}

\begin{tabular}{lll}
\hline Chapter 6. Cox rings of generalized Del Pezzo surfaces & 81
\end{tabular}

6.1. Introduction 81

$\begin{array}{ll}\text { 6.2. Generators } & 82\end{array}$

6.3. Relations 86

$\begin{array}{lll}6.4 . & \text { Degree 4 } & 89\end{array}$

\begin{tabular}{lll}
\hline 6.5. & Degree 3 & 93
\end{tabular}

6.6. Families of Del Pezzo surfaces 96

\begin{tabular}{|ll|}
\hline Part 2. & Rational points on Del Pezzo surfaces \\
\hline
\end{tabular}

$\begin{array}{lll}\text { Chapter 7. Manin's conjecture } & 101\end{array}$

$\begin{array}{ll}\text { 7.1. Introduction } & 101\end{array}$

$\begin{array}{lll}77.2 . & \text { Peyre's constant } & 105\end{array}$

\begin{tabular}{lll}
\hline 7.3. & Height zeta functions & 106
\end{tabular}

Chapter 8. On a constant arising in Manin's conjecture 109

8.1. Introduction 109

8.2. Smooth Del Pezzo surfaces 111

8.3. Singular Del Pezzo surfaces 113

$\begin{array}{lll}\text { Chapter 9. Manin's conjecture for a singular cubic surface } & 117\end{array}$

$\begin{array}{ll}9.1 . & \text { Introduction } \\ & 117\end{array}$

9.2. Manin's conjecture 118

$\begin{array}{lll}9.3 . & \text { The universal torsor } & 119\end{array}$

9.4. Congruences 123

9.5. Summations 124

$\begin{array}{lll}9.6 . & \text { Completion of the proof } & 129\end{array}$

Chapter 10. Manin's conjecture for a singular quartic surface 133

10.1. Introduction 133

$\begin{array}{lll}10.2 . & \text { Geometric background } & 134\end{array}$

10.3. Manin's conjecture 136

$\begin{array}{lll}10.4 . & \text { The universal torsor } & 136\end{array}$

10.5. Summations 140

10.6. Completion of the proof 144

\begin{tabular}{ll}
\hline Bibliography & 145
\end{tabular}

\begin{tabular}{ll}
\hline Index & 149
\end{tabular}

\begin{tabular}{ll}
\hline Lebenslauf & 151
\end{tabular} 


\section{Introduction}

The topic of this thesis is the geometry and arithmetic of Del Pezzo surfaces. Prime examples are cubic surfaces, which were already studied by Cayley, Schläfli, Steiner, Clebsch, and Cremona in the 19th century. Many books and articles followed, for example by Segre [Seg42 and Manin Man86.

On the geometric side, our main goal is to understand the geometry of universal torsors over Del Pezzo surfaces. On the arithmetic side, we apply universal torsors to questions about rational points on Del Pezzo surfaces over number fields. We are concerned with the number of rational points of bounded height in the context of Manin's conjecture [FMT89.

More precisely, a smooth cubic surface $S$ in three-dimensional projective space $\mathbb{P}^{3}$ over the field $\mathbb{Q}$ of rational numbers is defined by the vanishing of a non-singular cubic form $f \in \mathbb{Z}\left[x_{0}, x_{1}, x_{2}, x_{3}\right]$. Its rational points are

$$
S(\mathbb{Q}):=\left\{\mathbf{x}=\left(x_{0}: x_{1}: x_{2}: x_{3}\right) \in \mathbb{P}^{3}(\mathbb{Q}) \mid f(\mathbf{x})=0\right\} .
$$

If $S(\mathbb{Q}) \neq \emptyset$, then $S(\mathbb{Q})$ is dense with respect to the Zariski topology, i.e., there is no finite set of curves on $S$ containing all rational points.

One natural approach to understand $S(\mathbb{Q})$ is to ask how many rational points of bounded height there are on $S$. Here, the height of a point $\mathbf{x} \in S(\mathbb{Q})$, represented by coprime integral coordinates $x_{0}, x_{1}, x_{2}, x_{3}$, is

$$
H(\mathbf{x}):=\max \left\{\left|x_{0}\right|,\left|x_{1}\right|,\left|x_{2}\right|,\left|x_{3}\right|\right\} .
$$

The number of rational points on $S$ whose height is bounded by a positive number $B$ is

$$
N_{S, H}(B):=\#\{\mathbf{x} \in S(\mathbb{Q}) \mid H(\mathbf{x}) \leq B\} .
$$

As it is difficult to determine the exact number $N_{S, H}(B)$, our question is: How does $N_{S, H}(B)$ behave asymptotically as $B$ tends to infinity?

It is classically known that $S$ contains 27 lines defined over the algebraic closure of $\mathbb{Q}$. If a line $\ell \subset S$ is rational, i.e., defined over $\mathbb{Q}$, the number of rational points on $\ell$ bounded by $B$ behaves asymptotically as a non-zero constant multiple of $B^{2}$. The behavior of $N_{S, H}(B)$ is dominated by the rational points lying on rational lines, so we modify our question as follows:

QuESTION. Let $U$ be the complement of the lines on a smooth cubic surface $S$. How does

$$
N_{U, H}(B):=\#\{\mathbf{x} \in U(\mathbb{Q}) \mid H(\mathbf{x}) \leq B\}
$$

behave asymptotically, as $B \rightarrow \infty$ ? 
The answer to this question depends on the geometry of $S$. Suppose that $S$ is split, i.e., all 27 lines on $S$ are defined over $\mathbb{Q}$. Then Manin's conjectural answer is:

Conjecture. There is a positive constant c such that

$$
N_{U, H}(B) \sim c \cdot B \cdot(\log B)^{6},
$$

as $B \rightarrow \infty$.

This conjecture is open for smooth cubic surfaces. Analogous statements have been proved for some smooth Del Pezzo surfaces of degrees $\geq 5$ ([BT98, Bre02]).

An approach which is expected to lead to a proof of Manin's conjecture for Del Pezzo surfaces is the use of universal torsors. For the projective plane, this method is used as follows: In order to estimate the number of rational points $\mathbf{x} \in \mathbb{P}^{2}(\mathbb{Q})$ whose height $H(\mathbf{x})$ is bounded by $B$, we observe that these points are in bijection to the integral points

$$
\mathbf{y}=\left(y_{0}, y_{1}, y_{2}\right) \in \mathbb{A}^{3}(\mathbb{Z}) \backslash\{(0,0,0)\}
$$

which satisfy the coprimality condition

$$
\operatorname{gcd}\left(y_{0}, y_{1}, y_{2}\right)=1
$$

and the height condition

$$
\max \left\{\left|y_{0}\right|,\left|y_{1}\right|,\left|y_{2}\right|\right\} \leq B,
$$

up to identification of $\mathbf{y}$ and $\mathbf{- y}$. The number of these points $\mathbf{y}$ can be estimated using standard methods of analytic number theory.

There is a similar bijection between rational points of bounded height on a cubic surface $S$ and integral points on a certain affine variety $\mathcal{T}_{S}$, which is called the universal torsor (see Chapter 2 for the definition), subject to certain coprimality and height conditions.

We can establish such a bijection in two ways. On the one hand, this can be done by elementary transformations of the form $f$ defining $S$ such as introducing new variables which are common divisors of previous coordinates (see Chapter 9 for an example). This way of passing to the universal torsor has been used in the proof of Manin's conjecture for some Del Pezzo surfaces of other degrees, e.g., BB04. We will see that these transformations are motivated by the geometric structure of $S$.

On the other hand, we can compute universal torsors via Cox rings. The Picard group $\operatorname{Pic}(S)$ of isomorphy classes of line bundles on $S$ is an abelian group which is free of rank 7 if $S$ is a split cubic surface. Effective line bundles have global sections, and the global sections of all isomorphy classes of line bundles on $S$ can be given the structure of a ring, resulting in the Cox ring $\operatorname{Cox}(S)$ (see Chapter 2). Its generators and relations correspond to the coordinates and equations defining an affine variety $\mathbb{A}(S)$ which contains the universal torsor $\mathcal{T}_{S}$ as an open subset.

Batyrev and Popov BP04 have determined the Cox ring of smooth Del Pezzo surfaces of degree $\geq 3$. In the case of smooth cubic surfaces, this realizes the universal torsor as an open subset of a 9-dimensional variety, defined by 81 equations in 27-dimensional affine space (see Section 3.3). 
However, estimating the number of points on the universal torsor seems to be very hard in this case.

Schläfli [Sch63] and Cayley Cay69 classified singular cubic surfaces. For the cubic surface

$$
S_{1}: x_{1} x_{2}^{2}+x_{2} x_{0}^{2}+x_{3}^{3}=0
$$

with a singularity whose type is denoted as $\mathbf{E}_{6}$, Hassett and Tschinkel HT04 have calculated the Cox ring. It is a polynomial ring in 10 variables with one relation, resulting in a universal torsor which is a hypersurface in 10-dimensional affine space. One of our main results is (see Theorem 9.1):

TheOREM. Manin's conjecture holds for the $\mathbf{E}_{6}$ cubic surface $S_{1}$.

Joint work with de la Bretèche and Browning resulting in a more precise asymptotic formula for this surface will appear in [BBD05].

This thesis is organized in two parts. Part 1 is concerned with Cox rings and universal torsors of smooth Del Pezzo surfaces and of generalized Del Pezzo surfaces, i.e., minimal desingularizations of singular ones. We work over algebraically closed fields of characteristic 0 . In Chapter 1, we give an exposition of the structure and classification of smooth, singular, and generalized Del Pezzo surfaces. In Chapter 2, we recall the definition of universal torsors and Cox rings and collect some preliminary results on the generators and relations in the Cox ring of Del Pezzo surfaces.

Our main results concerning universal torsors and Cox rings of generalized Del Pezzo surfaces are:

(1) We calculate the Cox ring of smooth cubic Del Pezzo surfaces explicitly, using results of Batyrev and Popov [BP04 on the relations up to radical in $\operatorname{Cox}(S)$ for smooth Del Pezzo surfaces of degree $\geq 3$. We extend these results to surfaces of degree 2 and 1 (Theorem 3.2 ).

(2) We find all generalized Del Pezzo surfaces of degree $\geq 3$ whose universal torsor can be realized as a hypersurface in affine space, or equivalently, where the ideal of relations defining the Cox ring has only one generator (Theorem 5.1). We determine the Cox ring in these cases.

(3) We give a method to determine generators and the ideal of relations (up to radical) of the Cox ring of any generalized Del Pezzo surface of degree $\geq 2$ (Theorem 6.2 and Section 6.3).

Skorobogatov [Sko93] and Salberger observed that the universal torsor of a quintic Del Pezzo surface is an open subset of a Grassmannian.

Furthermore, for a smooth cubic surface $S$, the 27 coordinates of the affine space containing the universal torsor correspond to the lines on $S$. Their classes in the Picard group can be identified with the weights of a 27dimensional representation of the linear algebraic group associated to the root system $\mathbf{E}_{6}$.

Batyrev conjectured that this is reflected geometrically by an embedding of the universal torsor of $S$ in a certain homogeneous space associated to this representation, similar to the Grassmannian in the quintic case. A corresponding result was proved by Popov Pop01 in degree 4 . 
(4) We prove Batyrev's conjecture that universal torsors of smooth Del Pezzo surfaces of degree 3 or 2 can be embedded naturally in the affine cone over a homogeneous space associated to certain linear algebraic groups (Theorem 4.1).

In Part 2, we apply our results of Part 1 to Manin's conjecture for certain Del Pezzo surfaces. In Chapter 7, we give a detailed introduction to the usage of torsors towards Manin's conjecture for Del Pezzo surfaces.

Our main results concerning Manin's conjecture are:

(5) We give a formula for a certain factor of the leading constant, as proposed by Peyre Pey95, in Manin's conjecture for smooth and singular Del Pezzo surfaces $S$. It is the volume of a polyhedron related to the cone of effective divisor classes on $S$. Our formula allows to compute this constant directly from the degree and the types of singularities on $S$ (Theorem 8.3 and Theorem 8.5).

(6) We prove Manin's conjecture for a cubic surface with a singularity of type $\mathbf{E}_{6}$ (Theorem 9.1).

(7) We prove Manin's conjecture for a split quartic surface with a singularity of type $\mathbf{D}_{4}$ (Theorem 10.1). 
Part 1

\section{Universal torsors of Del Pezzo} surfaces 



\section{CHAPTER 1}

\section{Del Pezzo surfaces}

\subsection{Introduction}

This chapter gives an exposition of the structure and classification of smooth and singular Del Pezzo surfaces. For smooth Del Pezzo surfaces, a standard reference is [Man86, while [DP80] and [AN04 are modern accounts of the structure and classification of singular Del Pezzo surfaces.

Schläfli Sch63 and Cayley Cay69 classified singular cubic surfaces in the 1860's. Timms [Tim28 and Du Val [DV34] analyzed them more systematically.

The basic objects of our studies are surfaces. For our purposes, these are projective varieties of dimension 2 over a field $\mathbb{K}$. In this chapter, we assume that the ground field $\mathbb{K}$ has characteristic zero and is algebraically closed. For basic notions of algebraic geometry, we refer to Hartshorne's book [Har77].

We are mainly interested in the following geometric invariants of a smooth surface $S$ :

Picard group: A prime divisor is an irreducible curve on $S$. The free abelian group generated by the prime divisors is the divisor group $\operatorname{Div}(S)$. Its elements are called divisors; non-negative linear combinations of prime divisors are effective divisors. Considering divisors up to linear equivalence (cf. [Har77, Section II.6]) leads to the Picard group $\operatorname{Pic}(S)$ of divisor classes as a quotient of the divisor group.

Divisor classes correspond to line bundles, or invertible sheafs, on $S$; see [Har77, Section II.6] for details. We will freely go back and forth between these points of view; we will not distinguish between divisors and their classes whenever this cannot cause confusion.

Intersection form: For smooth prime divisors which intersect transversally, the intersection number is simply the number of intersection points. This can be extended to a bilinear form on $\operatorname{Div}(S)$ and induces the intersection form $(\cdot, \cdot)$ on $\operatorname{Pic}(S)$ (cf. Har77, Section V.1]). Let $(D, D)$ be the self intersetion number of a divisor (class) $D$.

Canonical class: The canonical class $K_{S}$ of a smooth surface $S$, as defined in [Har77, Section II.8], is the second exterior power of the sheaf of differentials on $S$. Its negative $-K_{S}$ is the anticanonical class. If $S$ is a singular normal (see [Har77, Exercise 3.17]) surface with rational double points (see [Art66]), we define its 
anticanonical class $-K_{S}$ such that its pull-back under a minimal desingularization $f: \widetilde{S} \rightarrow S$ is $-K_{\widetilde{S}}$.

Ampleness: A divisor class $L \in \operatorname{Pic}(S)$ is very ample if it defines an embedding of $S$ in projective space (see [Har77, Section II.5]), while $L^{\prime} \in \operatorname{Pic}(S)$ is ample if a positive multiple of $L^{\prime}$ is very ample.

Blow-ups: The blow-up of a point on a surface replaces this point in a particular way by a divisor which has self intersection number -1 and which is isomorphic to the projective line $\mathbb{P}^{1}$ (cf. Har77, Section I.4]).

In this chapter, we study the following classes of surfaces:

- A smooth Del Pezzo surface is a smooth surface whose anticanonical class is ample.

- A singular Del Pezzo surface is a singular normal surface whose singularities are rational double points and whose anticanonical class is ample.

- A generalized Del Pezzo surface is either a smooth Del Pezzo surface or the minimal desingularization of a singular Del Pezzo surface.

The degree of a generalized Del Pezzo surface $S$ is the self intersection number of its anticanonical class. Generalized Del Pezzo surfaces of degree $9-r \leq 7$ can be obtained by a sequence of $r$ blow-ups of $\mathbb{P}^{2}$.

This chapter is structured as follows: In Section 1.2, we study how blow-ups affect basic invariants of surfaces such as the Picard group with its intersection form and the anticanonical class. Section 1.3 is concerned with smooth Del Pezzo surfaces and their prime divisors with self intersection number -1 , which we call (-1)-curves. In Section 1.4, we describe how certain Weyl groups and root systems are connected to the configuration of $(-1)$-curves on smooth Del Pezzo surfaces.

Section 1.5 is concerned with singular and generalized Del Pezzo surfaces. The desingularization of singularities of singular Del Pezzo surfaces gives rise to prime divisors with self intersection number -2 , which we call $(-2)$ curves. In Section 1.6, we show how the $(-2)$-curves of a generalized Del Pezzo surface can be interpreted as the roots of root systems in the Picard group of a smooth Del Pezzo surface of the same degree, and how the (-1)curves of these Del Pezzo surfaces are related. This allows the classification of singular Del Pezzo surfaces. In Section 1.7, we explain how to recover configurations of blown-up points from the configuration of $(-1)$ - and (-2)curves on generalized Del Pezzo surfaces. In Section 1.8, we determine which generalized Del Pezzo surfaces are toric varieties [Ful93].

\subsection{Blow-ups of smooth surfaces}

A classical construction in algebraic geometry is the blow-up of a point on a surface.

Lemma 1.1. Suppose $S$ is a smooth surface. Let $\pi: S^{\prime} \rightarrow S$ be the blow-up of $p \in S$.

- The preimage $E:=\pi^{-1}(p)$ is isomorphic to $\mathbb{P}^{1}$.

- The map $\pi$ is a birational morphism which is an isomorphism between $S^{\prime} \backslash E$ and $S \backslash\{p\}$. 
- The blow-up increases the rank of the Picard group by one:

$$
\operatorname{Pic}\left(S^{\prime}\right) \cong \operatorname{Pic}(S) \oplus \mathbb{Z} .
$$

Here, $E=(0,1)$.

- Let $-K_{S}$ be the anticanonical class on $S$. Then $-K_{S^{\prime}}=\left(-K_{S},-1\right)$ is the anticanonical class on $S^{\prime}$.

In this context, we have the following terminology.

- The curve $E \subset S^{\prime}$ is called the exceptional divisor of the blow-up $\pi: S^{\prime} \rightarrow S$.

- For a prime divisor $D$ on $S$, the strict (or proper) transform of $D$ $\widehat{D}$ is the prime divisor which is the closure of $\pi^{-1}(D \backslash\{p\})$.

- Let $\alpha$ be the multiplicity of $p \in S$ on the prime divisor $D$. The total transform $D^{\prime}$ of $D$ is $D^{\prime}:=\widehat{D}+\alpha E$.

The canonical map $\pi^{*}: \operatorname{Pic}(S) \hookrightarrow \operatorname{Pic}\left(S^{\prime}\right)$ maps $L \in \operatorname{Pic}(S)$ to $L^{\prime}=(L, 0)$. If $L$ is the class of a divisor $D$, then $L^{\prime}$ is the class of the total transform $D^{\prime}$ of $D$. The class of the strict transform $\widehat{D}$ of $D$ is $(L,-\alpha)$.

Whenever we use the same symbol for a divisor on a surface $S$ and its blow-up $S^{\prime}$, it shall denote on $S^{\prime}$ the strict transform of the divisor on $S$, unless specified differently.

Lemma 1.2. Let $\pi: S^{\prime} \rightarrow S$ be the blow-up of $p \in S$. Using the isomorphism $\operatorname{Pic}\left(S^{\prime}\right) \cong \operatorname{Pic}(S) \oplus \mathbb{Z}$ as above, we have:

- The self intersection number of the exceptional divisor $E$ is -1 , and $E$ does not intersect the total transforms of divisors on $S$.

- Let $L_{1}=\left(M_{1}, \alpha_{1}\right)$ and $L_{2}=\left(M_{2}, \alpha_{2}\right)$, where $L_{i} \in \operatorname{Pic}\left(S^{\prime}\right), M_{i} \in$ $\operatorname{Pic}(S), \alpha_{i} \in \mathbb{Z}$. Then the intersection form on $\operatorname{Pic}\left(S^{\prime}\right)$ is

$$
\left(L_{1}, L_{2}\right)=\left(M_{1}, M_{2}\right)-\alpha_{1} \cdot \alpha_{2} .
$$

- In particular, if the multiplicity of $p$ on $D$ is $\alpha$, then $(\widehat{D}, \widehat{D})=$ $(D, D)-\alpha^{2}$, where $\widehat{D}$ is the strict transform of $D$.

A prime divisor $D$ with self intersection number $(D, D)=n$ is called $(n)$-curve. A negative curve is an $(n)$-curve with $n<0$. By Lemma 1.2 , blowing up points creates negative curves. They have the following property.

LEMma 1.3. Let $E$ be an effective divisor with negative self intersection number. Then the space of global sections of the corresponding line bundle $\mathcal{O}(E)$ has dimension $\operatorname{dim} H^{0}(S, \mathcal{O}(E))=1$.

Proof. Since $E$ is effective, $\mathcal{O}(E)$ has global sections.

Suppose $\operatorname{dim} H^{0}(S, \mathcal{O}(E)) \geq 2$. Then we can find two linearly independent sections $s_{1}, s_{2}$. As $(E, E)$ is the number of intersection points of the effective divisors corresponding to $s_{1}$ and $s_{2}$, it must be non-negative.

Therefore, the effective divisor corresponding to a divisor class with negative self intersection number is unique.

Remark 1.4. As explained in [HT04, Section 3], the Picard group is partially ordered: For $L, L^{\prime} \in \operatorname{Pic}(S)$, we have $L \preceq L^{\prime}$ if $L^{\prime}-L$ is an effective divisor class. If furthermore $L \neq L^{\prime}$, we write $L \prec L^{\prime}$. 
EXAmPLE 1.5. Consider the projective plane $\mathbb{P}^{2}$ over $\mathbb{K}$. Its Picard group $\operatorname{Pic}\left(\mathbb{P}^{2}\right)$ is isomorphic to $\mathbb{Z}$, where we identify the class of a line in $\mathbb{P}^{2}$ with $1 \in \mathbb{Z}$. We write $\mathcal{O}_{\mathbb{P}^{2}}(k)$ for the element of $\operatorname{Pic}\left(\mathbb{P}^{2}\right)$ corresponding to $k \in \mathbb{Z}$. The anticanonical class $-K_{\mathbb{P}^{2}} \in \operatorname{Pic}\left(\mathbb{P}^{2}\right)$ is $\mathcal{O}_{\mathbb{P}^{2}}(3)$. The intersection number of $\mathcal{O}_{\mathbb{P}^{2}}(a)$ and $\mathcal{O}_{\mathbb{P}^{2}}(b)$ is $a \cdot b$.

We are mainly interested in blow-ups of $\mathbb{P}^{2}$ in $r$ points.

Definition 1.6. For $1 \leq r \leq 8$, the blow-up $\pi: \widetilde{S}_{r} \rightarrow \mathbb{P}^{2}$ of $\mathbb{P}^{2}$ in $p_{1}, \ldots, p_{r}$ with exceptional divisors $E_{1}, \ldots, E_{r}$ is given as follows:

Let $\widetilde{S}_{0}:=\mathbb{P}^{2}$, and for $1 \leq i \leq r$, let

$$
\pi_{i}: \widetilde{S}_{i} \rightarrow \widetilde{S}_{i-1}
$$

be the blow-up of a point $p_{i} \in \widetilde{S}_{i-1}$, with exceptional divisor $E_{i}^{\prime}$ on $\widetilde{S}_{i}$. Then

$$
\pi:=\pi_{1} \circ \cdots \circ \pi_{r}: \widetilde{S}_{r} \rightarrow \mathbb{P}^{2},
$$

and $E_{i}$ is the strict transform of $E_{i}^{\prime}$ under $\pi_{i+1}, \ldots, \pi_{r}$.

LEMma 1.7. A standard basis of $\operatorname{Pic}\left(\widetilde{S}_{r}\right) \cong \mathbb{Z}^{r+1}$ is given by $l_{0}, \ldots, l_{r}$, where $l_{0}:=\pi^{*}\left(\mathcal{O}_{\mathbb{P}^{2}}(1)\right)$ and, for $i \in\{1, \ldots, r\}$,

$$
l_{i}:=\left(\pi_{i+1} \circ \cdots \circ \pi_{r}\right)^{*}\left(E_{i}^{\prime}\right)
$$

is the total transform of the exceptional divisor $E_{i}^{\prime}$ on $\widetilde{S}_{i}$. The intersection form is given by

$$
\left(l_{0}, l_{0}\right)=1, \quad\left(l_{1}, l_{1}\right)=\cdots=\left(l_{r}, l_{r}\right)=-1, \quad\left(l_{i}, l_{j}\right)=0 \text { if } i \neq j .
$$

It is non-degenerate. In terms of this basis,

$$
-K_{\widetilde{S}_{r}}=3 l_{0}-\left(l_{1}+\cdots+l_{r}\right)
$$

is the anticanonical class.

For an effective divisor $D$ on $\widetilde{S}_{j}$, we say that $D$ passes through $p_{k}$ (with $k>j$ ) if the strict transform of $D$ on $\widetilde{S}_{k-1}$ (respectively $D$ itself if $k=j+1$ ) contains $p_{k}$.

Note that $E_{r}=l_{r}$, but for $i<r$, the classes $E_{i}$ and $l_{i}$ (i.e., the strict and the total transform of the $i$-th exceptional divisor $E_{i}^{\prime}$ on $\widetilde{S}_{i}$ ) differ if $E_{i}^{\prime}$ passes through one of $p_{i+1}, \ldots, p_{r}$.

Consider the following convex cones Zie95 in

$$
\operatorname{Pic}\left(\widetilde{S}_{r}\right)_{\mathbb{R}}:=\operatorname{Pic}\left(\widetilde{S}_{r}\right) \otimes_{\mathbb{Z}} \mathbb{R} \cong \mathbb{R}^{r+1} .
$$

Definition 1.8. The effective cone $\Lambda_{\text {eff }}\left(\widetilde{S}_{r}\right)$ is the closed convex cone in $\operatorname{Pic}\left(\widetilde{S}_{r}\right)_{\mathbb{R}}$ generated by the classes of effective divisors.

The nef cone $\Lambda_{\text {eff }}^{\vee}\left(\widetilde{S}_{r}\right)$ is the closed convex cone generated by numerically effective, or nef divisor classes, i.e., divisor classes which have non-negative intersection number with all classes of effective divisors.

The nef cone is the dual of the effective cone with respect to the intersection form. By Kleiman's criterion [Kle66], a divisor class is ample if and only if it is in the interior of the nef cone. 


\subsection{Smooth Del Pezzo surfaces}

In this section, we describe basic properties of smooth Del Pezzo surfaces.

Definition 1.9. A smooth Del Pezzo surface is a smooth surface whose anticanonical class is ample.

For $1 \leq r \leq 8$, distinct points $p_{1}, \ldots, p_{r}$ in $\mathbb{P}^{2}$ are in general position if

- there is no line in $\mathbb{P}^{2}$ containing three of them,

- if $r \geq 6$, no conic in $\mathbb{P}^{2}$ contains six of them,

- if $r=8$, no cubic curve in $\mathbb{P}^{2}$ containing all eight points has a singularity in one of them.

Lemma 1.10. In the situation of Definition 1.6, the points $p_{1}, \ldots, p_{r}$ are in general position if and only if every prime divisor $D$ on $\widetilde{S}_{r}$ has self intersection number $(D, D) \geq-1$.

Proof. Consider how the self intersection numbers of prime divisors in $\mathbb{P}^{2}$ behave under blow-ups (Lemma 1.2). See also [DP80, Section II.3].

Theorem 1.11. A surface $S$ is a smooth Del Pezzo surface if and only if $S=\mathbb{P}^{2}$, or $S=\mathbb{P}^{1} \times \mathbb{P}^{1}$, or $S$ is the blow-up $S_{r}$ of $\mathbb{P}^{2}$ in $r \leq 8$ points in general position. The degree is nine for $\mathbb{P}^{2}$, eight for $\mathbb{P}^{1} \times \mathbb{P}^{1}$, and $9-r$ for $S_{r}$.

Proof. See [Man86, Theorem 24.4].

REMARK 1.12. In the notation of Definition 1.6 and Lemma 1.7, the strict and total transforms $E_{i}$ and $l_{i}$ of $E_{i}^{\prime}$ coincide if $p_{1}, \ldots, p_{r}$ are in general position. In this case, we usually use the notation $S_{i}:=\widetilde{S}_{i}$ and $H:=l_{0}$, so the standard basis is given by $H, E_{1}, \ldots, E_{r}$.

If $D \in \operatorname{Pic}(S)$ is an effective divisor on a Del Pezzo surface $S$, then $\left(-K_{S}, D\right)>0$. By Lemma 1.10 , the only negative curves on $S$ are $(-1)$ curves. By Man86. Theorem 24.3], we have $\left(D,-K_{S}\right)=1$ and $D \cong \mathbb{P}^{1}$ exactly when $D$ is a $(-1)$-curve. The proof uses the adjunction formula [Har77, Proposition V.1.5].

REMARK 1.13. For $r \leq 6$, the anticanonical class $-K_{S_{r}}$ is $\phi_{r}^{*}\left(\mathcal{O}_{\mathbb{P}^{9-r}}(1)\right)$ for the anticanonical embedding

$$
\phi_{r}: S_{r} \hookrightarrow \mathbb{P}^{9-r} .
$$

The $(-1)$-curves on $S_{r}$ are mapped exactly to the lines on $\phi_{r}\left(S_{r}\right) \subset \mathbb{P}^{9-r}$.

The image $\phi_{6}\left(S_{6}\right)$ of degree 3 in $\mathbb{P}^{3}$ is given by a non-singular cubic form, while $\phi_{5}\left(S_{5}\right)$ of degree 4 in $\mathbb{P}^{4}$ is the intersection of two quadrics.

There are no $(-1)$-curves on the Del Pezzo surfaces $\mathbb{P}^{2}$ and $\mathbb{P}^{1} \times \mathbb{P}^{1}$.

LEMma 1.14. Let $S_{r}$ be the blow-up of $p_{1}, \ldots, p_{r} \in \mathbb{P}^{2}$ in general position. The $(-1)$-curves on $S_{r}$ are the exceptional divisor $E_{1}, \ldots, E_{r}$ of the blow-up and the strict transforms of the following curves in $\mathbb{P}^{2}$ :

- If $r \geq 2$, the lines in $\mathbb{P}^{2}$ through two of the blown-up points.

- If $r \geq 5$, the conics through five of the blown-up points.

- If $r \geq 7$, the cubics through seven blown-up points, one of them a double point of the cubic. 
- If $r=8$, the quartics through the eight points $p_{i}$, three of them double points.

- If $r=8$, the quintics through the eight points, six of them double points.

- If $r=8$, the sextics through the eight points, seven of them double and the eighth a triple point of the sextic.

The number $N_{r}$ of $(-1)$-curves on $S_{r}$ can be found in Table 1.1.

Proof. See Man86, Theorem 26.2].

\begin{tabular}{|c||c|c|c|c|c|c|c|c|}
\hline$r$ & 1 & 2 & 3 & 4 & 5 & 6 & 7 & 8 \\
\hline \hline$N_{r}$ & 1 & 3 & 6 & 10 & 16 & 27 & 56 & 240 \\
\hline
\end{tabular}

TABLE 1.1. The number of $(-1)$-curves on $S_{r}$.

The class $D \in \operatorname{Pic}\left(S_{r}\right)$ of the strict transform of a curve $D^{\prime}$ in $\mathbb{P}^{2}$ of degree $d$ which has multiplicity $\lambda_{i}$ in $p_{i}$ for $i \in\{1, \ldots, r\}$ is

$$
D=d H-\sum_{i=1}^{r} \lambda_{i} E_{i}
$$

Lemma 1.15. For $2 \leq r \leq 8$, let $S_{r}$ be a smooth Del Pezzo surface of degree $9-r$. The $(-1)$-curves on $S_{r}$ generate the effective cone $\Lambda_{\mathrm{eff}}\left(S_{r}\right)$.

Proof. See [BP04, Corollary 3.3].

REMARK 1.16. For $2 \leq r \leq 7$, the semigroup of effective divisor classes in $\operatorname{Pic}\left(S_{r}\right)$ is generated by the $(-1)$-curves, i.e., every effective divisor class is a non-negative linear combination of the $(-1)$-curves. However, for $r=8$, this is only true for every effective divisor class besides the anticanonical class $-K_{S_{8}}($ BP04, Corollary 3.3]).

Remark 1.17. By [Har77, Example II.7.1.1], the automorphisms of $\mathbb{P}^{2}$ are given by the action of elements of $\mathrm{PGL}_{3}(\mathbb{K})=\mathrm{GL}_{3}(\mathbb{K}) / \mathbb{K}^{*}$ of invertible $3 \times 3$-matrices over $\mathbb{K}$ up to non-zero multiple. For every set of four points in general position in $\mathbb{P}^{2}$, we can find a unique element of $\mathrm{PGL}_{3}(\mathbb{K})$ which maps them to four arbitrarily chosen points in general position. Hence, for $r \geq 4$ points $p_{1}, \ldots, p_{r}$ in general position, we may assume that

$p_{1}=(1: 0: 0), \quad p_{2}=(0: 1: 0), \quad p_{3}=(0: 0: 1), \quad p_{4}=(1: 1: 1)$.

Furthermore, the requirements for points in general position show that the remaining points must have non-zero coordinates.

This also shows that smooth Del Pezzo surfaces of degree $\geq 5$ are unique up to isomorphism, while in degree $9-r \leq 4$, we have a $(2 \cdot(r-4))$-parameter family of isomorphy classes of smooth Del Pezzo surfaces.

\subsection{Weyl groups and root systems}

The configuration of 27 lines on a smooth cubic surface is preserved under the action of the Weyl group associated to the root system $\mathbf{E}_{6}$. Similar symmetries are associated to smooth Del Pezzo surfaces in other degrees. 
Recall the definition of root systems and Weyl groups, for example from Bro89]. Consider $\mathbb{R}^{s}$ with a non-degenerate bilinear form $(\cdot, \cdot)$. For $D \in \mathbb{R}^{s}$, let

$$
D^{=k}:=\left\{D^{\prime} \in \mathbb{R}^{s} \mid\left(D^{\prime}, D\right)=k\right\}
$$

denote the hyperplane of elements of $\mathbb{R}^{s}$ whose pairing with $D$ is $k$. Then

$$
D^{\geq k}:=\left\{D^{\prime} \in \mathbb{R}^{s} \mid\left(D^{\prime}, D\right) \geq k\right\}
$$

is the closed positive halfspace defined by $D^{=k}$, while $D^{>k}:=D^{\geq k} \backslash D^{=k}$ is the open positive halfspace. Similarly, $D^{\leq k}$ (resp. $D^{<k}$ ) defines the closed (resp. open) negative halfspace.

A finite set $\Phi \subset \mathbb{R}^{s}$ is a root system if it is invariant under the reflections $s_{\alpha}$ on $\alpha^{=0}$ for any $\alpha \in \Phi$, defined as

$$
s_{\alpha}(x):=x-\frac{2(x, \alpha)}{(\alpha, \alpha)} \cdot \alpha .
$$

The elements of $\Phi$ are called roots. The rank of $\Phi$ is the dimension of the subspace of $\mathbb{R}^{s}$ which is generated by $\Phi$.

For some $D \in \mathbb{R}^{s}$ such that $D^{=0} \cap \Phi=\emptyset$, let $\Phi^{+}=\Phi \cap D^{>0}$ be the set of positive roots in $\Phi$. For any $\alpha \in \Phi$, we have either $\alpha \in \Phi^{+}$or $-\alpha \in \Phi^{+}$. Furthermore, we can find a minimal system $\Delta$ of positive roots such that any $\alpha \in \Phi^{+}$is a non-negative linear combination of elements of $\Delta$. The elements of $\Delta$ are called simple roots. A root system $\Phi$ is represented by the Dynkin diagram of its simple roots (see Remark 1.18).

The reflections $s_{\alpha}$ for $\alpha \in \Phi$ generate the finite Weyl group $W$ which acts on $\mathbb{R}^{s}$. It acts transitively on $\Phi$, and it is already generated by all $s_{\alpha}$ for $\alpha \in \Delta$.

The hyperplanes $\alpha^{=0}$ for $\alpha \in \Phi^{+}$divide $\mathbb{R}^{s}$ into chambers (cf. Bro89, Section I.4B]). The (closed) fundamental chamber is

$$
\mathcal{C}_{0}:=\left\{x \in \mathbb{R}^{s} \mid(x, \alpha) \geq 0 \text { for all } \alpha \in \Delta\right\}=\bigcap_{\alpha \in \Delta} \alpha^{\geq 0} .
$$

All other (closed) chambers have the form $\mathcal{C}_{w}:=w\left(\mathcal{C}_{0}\right)$ for some $w \in W$. The set of chambers is in bijection to the set of elements of $W$. The space $\mathbb{R}^{s}$ is the union of all chambers, and the dimension of the intersection of two chambers is smaller than $s$.

Let $S_{r}$ be a smooth Del Pezzo surface which is the blow-up of $\mathbb{P}^{2}$ in $r$ points in general position. For $3 \leq r \leq 8$ and $i \in\{1, \ldots, r\}$, let

$$
\alpha_{i}:= \begin{cases}E_{i}-E_{i+1}, & i \leq 2, \\ H-E_{1}-E_{2}-E_{3}, & i=3, \\ E_{i-1}-E_{i}, & i \geq 4,\end{cases}
$$

using the basis of $\operatorname{Pic}\left(S_{r}\right)$ of Remark 1.12 .

Then $\alpha_{1}, \ldots, \alpha_{r}$ have self intersection number -2 . They are the simple roots $\Delta_{r}$ of a root system $\Phi_{r}$ of rank $r$ in $\operatorname{Pic}\left(S_{r}\right)_{\mathbb{R}}$. The roots of $\Phi_{r}$ are $E-E^{\prime}$ for any pair of distinct non-intersecting $(-1)$-curves $E, E^{\prime}$.

For $r=2$, there are exactly two roots $E_{1}-E_{2}$ and $E_{2}-E_{1}$, giving rise to the root system $\Phi_{2}$. For $r=1$, there is no root system. 
Remark 1.18. We denote Dynkin diagram as follows. The symbols $\mathbf{A}_{n}$, $\mathbf{D}_{n}$, and $\mathbf{E}_{n}$ are associated to the following diagrams:

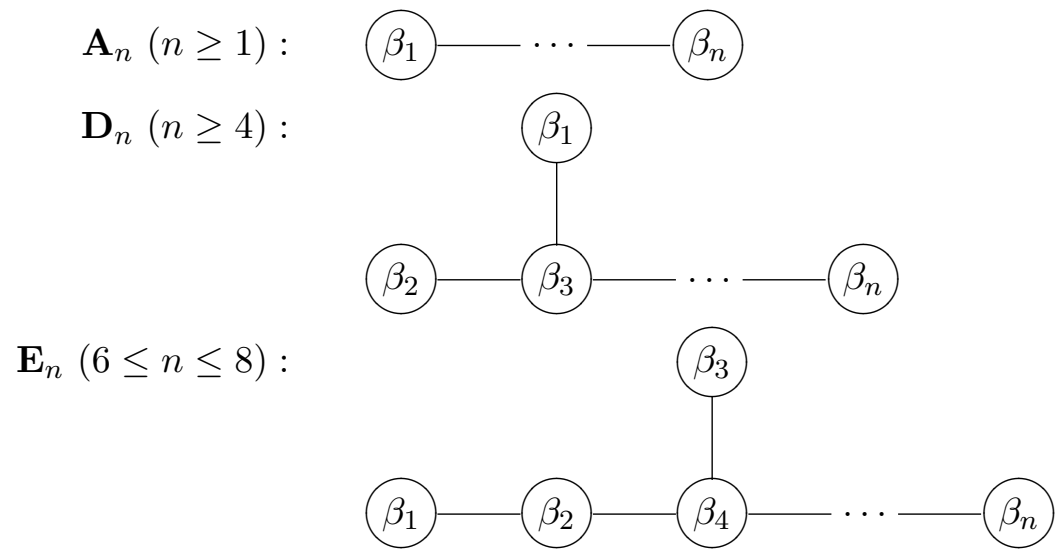

They contain $n$ vertices corresponding to $n$ simple roots $\beta_{1}, \ldots, \beta_{n}$ of an irreducible root system of rank $n$. The intersection number of two distinct simple roots is the number of edges between the corresponding vertices; the self intersection number of a root is -2 .

If a root system is the orthogonal sum of irreducible root systems, we denote its type as a sum of the symbols corresponding to its irreducible components.

See Table 1.2 for the types of $\Phi_{r}$.

\begin{tabular}{|c||c|c|c|c|c|c|c|}
\hline$r$ & 2 & 3 & 4 & 5 & 6 & 7 & 8 \\
\hline$\Phi_{r}$ & $\mathbf{A}_{1}$ & $\mathbf{A}_{2}+\mathbf{A}_{1}$ & $\mathbf{A}_{4}$ & $\mathbf{D}_{5}$ & $\mathbf{E}_{6}$ & $\mathbf{E}_{7}$ & $\mathbf{E}_{8}$ \\
\hline
\end{tabular}

TABLE 1.2. Root systems associated to $S_{r}$.

For each root $\alpha \in \Phi_{r}$, the reflection $s_{\alpha}$ is given by

$$
s_{\alpha}(x)=x+(x, \alpha) \cdot \alpha,
$$

since $(\alpha, \alpha)=-2$. These reflections generate a Weyl group $W_{r}$. The intersection form is invariant under the action of $W_{r}$. Therefore, $W_{r}$ acts on the set of $(-1)$-curves on $S_{r}$. The anticanonical class $-K_{S_{r}}$ is invariant under this action.

Lemma 1.19. The Weyl group $W_{r}$ acts transitively on the following sets:

- The set of $(-1)$-curves on $S_{r}$, if $r \geq 3$.

- The set $\Phi_{r}$ of roots of $S_{r}$, if $r \geq 2$.

- The set of s-element sets of $(-1)$-curves which are pairwise nonintersecting, if $r \geq 2$ and $s \neq r-1$.

- The set of pairs of (-1)-curves which have intersection number 1, if $r \geq 2$.

Proof. For the first three statements, see [DP80, II, Theorem 2 and Proposition 4]. The last statement follows from [FM02, Lemma 5.3] for $r \geq 4$ (see also [BP04, Remark 4.7]). For $r \in\{2,3\}$, we can check it directly. 


\subsection{Singular Del Pezzo surfaces}

We study blow-ups of $\mathbb{P}^{2}$ as in Definition 1.6 in the following configurations of points:

DeFinition 1.20. The points $p_{1}, \ldots, p_{r}$ are in almost general position if and only if:

- No line in $\mathbb{P}^{2}$ passes through four of the points $p_{1}, \ldots, p_{r}$.

- No conic in $\mathbb{P}^{2}$ passes through seven of the points.

- None of the exceptional divisors $E_{1}^{\prime}, \ldots, E_{r}^{\prime}$ passes through two of the points.

Lemma 1.21. The points $p_{1}, \ldots, p_{r}$ are in almost general position if and only if the following equivalent conditions hold:

- For $i \in\{1, \ldots, r\}$, the point $p_{i}$ does not lie on a $(-2)$-curve of $\widetilde{S}_{i-1}$.

- Every prime divisor $D$ on $\widetilde{S}_{r}$ satisfies $(D, D) \geq-2$.

Proof. For the first condition, see [DP80, Theorem III.1], which also lists further equivalent conditions. The second follows from Lemma 1.2 .

Definition 1.22. A singular Del Pezzo surface is a singular normal surface $S$ whose singularities are rational double points, and whose anticanonical class $-K_{S}$ is ample.

A generalized Del Pezzo surface is either a smooth Del Pezzo surface or the minimal desingularization of a singular Del Pezzo surface.

Note that every generalized Del Pezzo surface is smooth. However, the term "smooth Del Pezzo surface" as introduced in Definition 1.9 refers to generalized Del Pezzo surfaces which do not contain (-2)-curves, i.e., which are not minimal desingularizations of singular Del Pezzo surfaces.

Rational double points (see [Art66] and [CT88, Proposition 0.1]) are singularities which can be resolved by a finite sequence of blow-ups of singular points, giving a minimal desingularization $f: \widetilde{S} \rightarrow S$, with smooth $\widetilde{S}$ and $-K_{\widetilde{S}}=f^{*}\left(-K_{S}\right)$. The blow-ups produce exceptional divisors which are $(-2)$-curves on $\widetilde{S}$. The exceptional divisors are the simple roots of a root system in $\operatorname{Pic}(\widetilde{S})_{\mathbb{R}}$ with associated Dynkin diagram as in Remark 1.18. We label the singularities using the symbols of their Dynkin diagrams (e.g., we say that " $S$ has a singularity of type $\mathbf{A}_{1}$ "). On singular Del Pezzo surfaces, singularities of types $\mathbf{A}_{n}(1 \leq n \leq 8), \mathbf{D}_{n}(4 \leq n \leq 8)$, and $\mathbf{E}_{n}(6 \leq n \leq 8)$ occur.

Definition 1.23. For two singular Del Pezzo surfaces of the same degree, we say that they have the same singularity type if and only if the number and types of their singularities coincide. It is denoted using the names of the corresponding Dynkin diagrams.

The singularity type of a generalized Del Pezzo surface which is not a smooth Del Pezzo surface is the singularity type of the corresponding singular one.

For example, the singularity type of a Del Pezzo surface with two singularities of type $\mathbf{A}_{1}$, one singularity of type $\mathbf{A}_{3}$, and no other singularity is denoted as $2 \mathbf{A}_{1}+\mathbf{A}_{3}$. 
EXAmPLE 1.24. The Hirzebruch surface $F_{2}:=\mathbb{P}\left(\mathcal{O}_{\mathbb{P}^{1}} \oplus \mathcal{O}_{\mathbb{P}^{1}}(-2)\right)$ is a generalized Del Pezzo surface of degree 8 whose singularity type is $\mathbf{A}_{1}$. For more details, see [Ful93, Section 1.1].

TheORem 1.25. Every generalized Del Pezzo surface is either $\mathbb{P}^{2}$, or $\mathbb{P}^{1} \times \mathbb{P}^{1}$, or the Hirzebruch surface $F_{2}$, or the blow-up $\pi: \widetilde{S}_{r} \rightarrow \mathbb{P}^{2}$ in $r \leq 8$ points $p_{1}, \ldots, p_{r}$ in almost general position.

Proof. See [AN04.

If $D \in \operatorname{Pic}(S)$ is an effective divisor on a generalized Del Pezzo surface $S$, then $\left(-K_{S}, D\right) \geq 0$. A negative curve $D$ fulfills $D \cong \mathbb{P}^{1}$ and is either a $(-1)$-curve with $\left(-K_{S}, D\right)=1$, or a $(-2)$-curve with $\left(-K_{S}, D\right)=0$ by the adjunction formula. Furthermore, if $\left(-K_{S}, D\right)=0$ for a prime divisor $D$, then $D$ is a $(-2)$-curve.

Let $\widetilde{S}_{r}$ be the blow-up of $\mathbb{P}^{2}$ in $r$ points in almost general position. The $(-2)$-curves on $\widetilde{S}_{r}$ are the strict transforms of:

- the exceptional divisors $E_{1}^{\prime}, \ldots, E_{r}^{\prime}$ which pass through one of the points $p_{1}, \ldots, p_{r}$,

- if $r \geq 3$, the lines in $\mathbb{P}^{2}$ which pass through three of $p_{1}, \ldots, p_{r}$,

- if $r \geq 6$, the conics in $\mathbb{P}^{2}$ which pass through six of these points,

- if $r=8$, the cubics in $\mathbb{P}^{2}$ which pass through seven of these points, with a singularity in the eighth.

We will see in Proposition 8.11 that the effective cone of every generalized Del Pezzo surface of degree $\leq 7$ is generated by its negative curves.

Remark 1.26. For $r \leq 6$, the anticanonical class $-K_{S_{r}}$ of a singular Del Pezzo surface $S_{r}$ is $\phi_{r}^{*}\left(\mathcal{O}_{\mathbb{P}^{9-r}}(1)\right)$ for the anticanonical embedding

$$
\phi_{r}: S_{r} \hookrightarrow \mathbb{P}^{9-r} \text {. }
$$

Let $f: \widetilde{S}_{r} \rightarrow S_{r}$ be the minimal desingularization. Since $-K_{\widetilde{S}_{r}}=f^{*}\left(-K_{S_{r}}\right)$, the morphism

$$
\phi_{r} \circ f: \widetilde{S}_{r} \rightarrow \mathbb{P}^{9-r}
$$

contracts exactly the $(-2)$-curves on $\widetilde{S}_{r}$ to the singularities of $S_{r}$ and maps the $(-1)$-curves to the lines on the image of $\phi_{r}$.

The image $\phi_{6}\left(S_{6}\right)$ of a singular cubic surface in $\mathbb{P}^{3}$ is given by a singular cubic form, while $\phi_{5}\left(S_{5}\right)$ of degree 4 in $\mathbb{P}^{4}$ is the intersection of two quadrics.

An important invariant of a generalized Del Pezzo surface is its extended Dynkin diagram, i.e., the configuration of the negative curves: We have a vertex for each negative curve, and the number of edges between two vertices is the intersection number of the corresponding negative curves. In our diagrams, we mark the (-2)-curves using circles.

EXAMPLE 1.27. By the classification of singular cubic surfaces BW79, there is a unique cubic surface $S_{6}$ with a singularity of type $\mathbf{E}_{6}$. Its anticanonical embedding $\phi_{6}\left(S_{6}\right) \subset \mathbb{P}^{3}$ is given by the vanishing of

$$
f(\mathbf{x})=x_{1} x_{2}^{2}+x_{2} x_{0}^{2}+x_{3}^{3} .
$$

It has one line $\left\{x_{2}=x_{3}=0\right\}$ containing the singularity $(0: 1: 0: 0)$. Its minimal desingularization $\widetilde{S}_{6}$ contains six $(-2)$-curves $E_{1}, \ldots, E_{6}$, and the 
transform of the line is a (-1)-curve $E_{7}$. The configuration of $E_{1}, \ldots, E_{6}$ is described by the Dynkin diagram $\mathbf{E}_{6}$, but for the extended Dynkin diagram, we also need to know how $E_{7}$ intersects the (-2)-curves .

By calculating the desingularization of $S_{6}$ explicitly, we see that the extended Dynkin diagram is as follows:

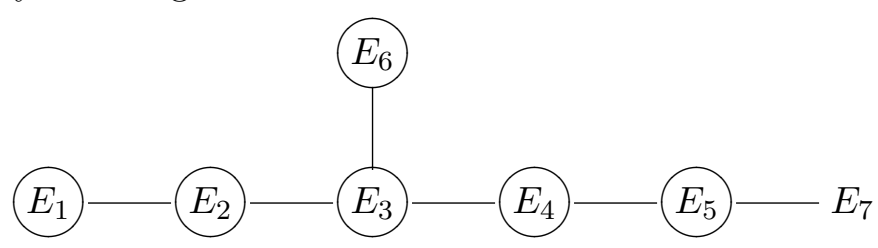

Definition 1.28. We say that two generalized Del Pezzo surfaces have the same type if and only if their degrees coincide and their extended Dynkin diagrams are isomorphic. Two singular Del Pezzo surfaces have the same type if and only if their minimal desingularizations have the same type.

See Remark 1.34 for a discussion how type and singularity type of Del Pezzo surfaces are related and how we denote the type.

\subsection{Classification of singular Del Pezzo surfaces}

We describe how to obtain extended Dynkin diagrams of all generalized Del Pezzo surfaces. We will use the results of this section in Chapter 5 , Chapter 6, and especially Chapter 8 .

EXAMPLE 1.29. In degree 3, consider the following extended Dynkin diagrams:

- The $\mathbf{E}_{6}$-diagram as in Example 1.27

- A diagram of type $\mathbf{A}_{5}+\mathbf{A}_{1}$ :

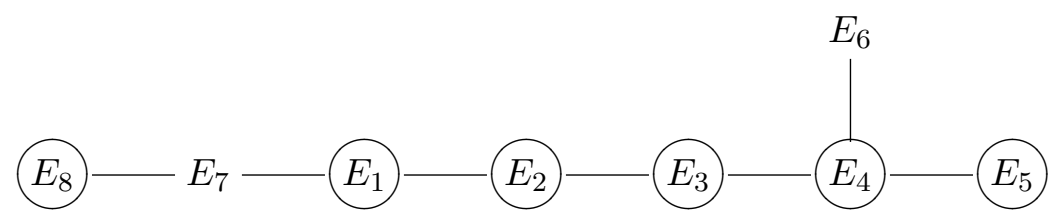

- A diagram of type $3 \mathbf{A}_{2}$ :

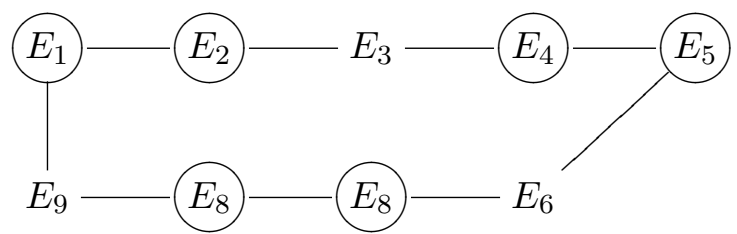

We will see how the extended Dynkin diagram of every generalized cubic Del Pezzo surface can be obtained from one of these three diagrams.

In general, we proceed as follows (see AN04 for proofs): Choose one of the extended Dynkin diagrams $\Gamma$ of [AN04, Table 3] whose number $N$ is between 4 and 10. They correspond to degrees $11-N$ from 7 to 1 , with more than one diagram to choose from for degrees 4 to 1 . Let $r:=N-2$.

For example, for $N=8$, i.e., degree 3 and $r=6$, we have the three diagrams of Example 1.29 to choose from.

The diagram $\Gamma$ describes the classes of some elements of $\mathbb{Z}^{r+1}$ together with their intersection numbers: Each of the $n$ vertices in $\Gamma$ corresponds to 
a basis element $e_{i}$ of a lattice $M:=\bigoplus_{i=1}^{n} \mathbb{Z} e_{i}$ with a bilinear form, where $\left(e_{i}, e_{i}\right)=-2$ if $e_{i}$ corresponds to a $(-2)$-curve in $\Gamma$ (represented by a black vertex in [AN04, Table 3], respectively marked by a circle in our notation), or $\left(e_{i}, e_{i}\right)=-1$ if $e_{i}$ corresponds to a $(-1)$-curve in $\Gamma$ (represented by a transparent vertex, respectively without a circle), and $\left(e_{i}, e_{j}\right)$ is the number of edges between the corresponding vertices for $i \neq j$. This intersection form on $M$ has rank $r+1$. Let $K$ be the kernel of this form. Let $E_{i}$ be the image of $e_{i}$ in $M / K \cong \mathbb{Z}^{r+1}$. The induced form $(\cdot, \cdot)$ on $\mathbb{Z}^{r+1}$ is a non-degenerate bilinear form, and the intersection behavior of $E_{1}, \ldots, E_{n}$ is described by $\Gamma$.

The $E_{i}$ with $\left(E_{i}, E_{i}\right)=-2$ form the simple roots $\Delta_{0}$ of a root system $\Phi_{0}$ in $\mathbb{R}^{r+1}$, generating a Weyl group $W_{0}$. Let $\mathcal{E}_{0}$ be the set of $E_{i}$ with self intersection number -1 .

Consider the orbit $\mathcal{E}$ of $\mathcal{E}_{0} \subset \mathbb{Z}^{r+1}$ under $W_{0}$ : We can find an isomorphism $\mathbb{Z}^{r+1} \cong \operatorname{Pic}\left(S_{r}\right)$ such that $\mathcal{E}$ is the set of $(-1)$-curves of $S_{r}$, and $\Phi_{0}$ is a root system in $\operatorname{Pic}\left(S_{r}\right)_{\mathbb{R}}$.

REMARK 1.30. Note that $W_{0}$ is equal to the Weyl group $W_{r}$ associated to the root system $\Phi_{r}$ listed in Table 1.2 only if we choose the first diagram in the list for $N=r+2$ in [AN04, Table 3].

To construct the extended Dynkin diagram $\Gamma(\widetilde{S})$ of the minimal desingularization $\widetilde{S}$ of a singular Del Pezzo surface $S$, choose a subset $\Delta$ of $\Delta_{0}$. These are the simple roots of a root system $\Phi \subset \Phi_{0}$, with positive roots $\Phi^{+} \subset \Phi_{0}^{+}$. The reflections $s_{\alpha}$ on the hyperplanes $\alpha^{=0}$ for the simple roots $\alpha \in \Delta$ generate a Weyl group $W \subset W_{0}$, with corresponding fundamental chamber $\mathcal{C}_{0}$.

Theorem 1.31. For every choice of $\Gamma$ from [AN04, Table 3] with $N \in$ $\{4, \ldots, 10\}$ (with corresponding simple roots $\Delta_{0}$ and $(-1)$-curves $\mathcal{E}$ ), and every choice of $\Delta \subset \Delta_{0}$ (giving a root system $\Phi$ with fundamental chamber $\left.\mathcal{C}_{0}\right)$, there is a generalized Del Pezzo surface $\widetilde{S}$ of degree $11-N$ whose $(-2)$ curves are $\Delta$ and whose $(-1)$-curves are $\mathcal{E} \cap \mathcal{C}_{0}$.

The extended Dynkin diagram of every generalized Del Pezzo surface of degree $\leq 7$ is obtained this way.

Proof. See AN04.

Example 1.32. Consider the diagram of type $\mathbf{A}_{5}+\mathbf{A}_{1}$ of Example 1.29 corresponding to $r=6$. This gives the following $8 \times 8$ intersection matrix:

\begin{tabular}{c|ccccccc|c} 
& $E_{1}$ & $E_{2}$ & $E_{3}$ & $E_{4}$ & $E_{5}$ & $E_{6}$ & $E_{7}$ & $E_{8}$ \\
\hline$E_{1}$ & -2 & 1 & 0 & 0 & 0 & 0 & 1 & 0 \\
$E_{2}$ & 1 & -2 & 1 & 0 & 0 & 0 & 0 & 0 \\
$E_{3}$ & 0 & 1 & -2 & 1 & 0 & 0 & 0 & 0 \\
$E_{4}$ & 0 & 0 & 1 & -2 & 1 & 1 & 0 & 0 \\
$E_{5}$ & 0 & 0 & 0 & 1 & -2 & 0 & 0 & 0 \\
$E_{6}$ & 0 & 0 & 0 & 1 & 0 & -1 & 0 & 0 \\
$E_{7}$ & 1 & 0 & 0 & 0 & 0 & 0 & -1 & 1 \\
\hline$E_{8}$ & 0 & 0 & 0 & 0 & 0 & 0 & 1 & -2
\end{tabular}

We check that the rank of the intersection matrix is $r+1=7$, and that the $7 \times 7$ submatrix $A$ of the first seven rows and columns has determinant 
with absolute value 1. Consequently, we can perform our computations in the basis $E_{1}, \ldots, E_{7}$, and $E_{8}$ can be expressed in terms of this basis using the intersection numbers between $E_{8}$ and $E_{1}, \ldots, E_{7}$ : As $E_{8}$ intersects only $E_{7}$, we have $E_{8}=A^{-1} \cdot(0,0,0,0,0,0,1)=(-1,0,1,2,1,2,-2)$.

The elements $E_{1}, \ldots, E_{5}, E_{8} \in \mathbb{Z}^{7}$ are the simple roots $\Delta_{0}$ of a root system $\Phi_{0}$ of type $\mathbf{A}_{5}+\mathbf{A}_{1}$. The reflections $s_{E_{i}}$ on $E_{i}^{=0}$ for $i \in\{1, \ldots, 5,8\}$ generate a Weyl group $W_{0}$. The orbit $\mathcal{E}$ of $\mathcal{E}_{0}=\left\{E_{6}, E_{7}\right\}$ under $W_{0}$ has $N_{6}=27$ elements. We can identify $\mathbb{Z}^{7}$ with $\operatorname{Pic}\left(S_{6}\right)$ together with the intersection forms such that $\mathcal{E}$ is the set of $(-1)$-curves of a smooth cubic surface $S_{6}$, and $\Phi_{0}$ is a root system in $\operatorname{Pic}\left(S_{6}\right)$. However, it is not the standard root system $\Phi_{6}$ of type $\mathbf{E}_{6}$ associated to this configuration of 27 $(-1)$-curves.

The Dynkin diagrams of various generalized Del Pezzo surfaces can be constructed by choosing subsets $\Delta$ of $\Delta_{0}$, for example:

- Let $\Delta:=\Delta_{0}$. The $(-1)$-curves in the closed fundamental chamber $\mathcal{C}_{0}$ corresponding to $\Delta_{0}$ are exactly $\mathcal{E}_{0}=\left\{E_{6}, E_{7}\right\}$. The extended Dynkin diagram of a generalized Del Pezzo surface $\widetilde{S}$ of type $\mathbf{A}_{5}+$ $\mathbf{A}_{1}$ is exactly the diagram $\Gamma$ that we started with.

- Let $\Delta:=\emptyset$. Then $\mathcal{C}_{0}$ is $\operatorname{Pic}\left(S_{6}\right)_{\mathbb{R}}$, and we obtain the diagram of a smooth cubic surface $S_{6}$ containing $27(-1)$-curves.

- Let $\Delta:=\left\{E_{1}, E_{2}, E_{4}, E_{5}\right\}$. In this case, the (-2)-curves $\Delta$ have the configuration $2 \mathbf{A}_{1}$, while $\mathcal{E} \cap \mathcal{C}_{0}$ consists of exactly seven $(-1)$ curves. The extended Dynkin diagram $\Gamma(\widetilde{S})$ of the corresponding generalized Del Pezzo surface of type $2 \mathbf{A}_{2}$ is:

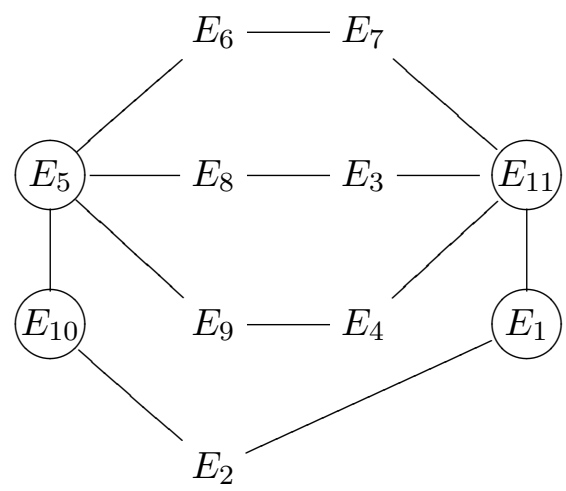

Here, we use the symbols $E_{1}, \ldots, E_{11}$ for the eleven negative curves in a way which is not related to the previous usage of $E_{1}, \ldots, E_{8}$.

We can check that the possible choices of $\Delta \subset \Delta_{0}$, where $\Delta_{0}$ is extracted from one of the three diagrams of Example 1.29, lead to exactly 21 nonisomorphic extended Dynkin diagrams. They correspond to the smooth cubic type and minimal desingularizations of 20 singular cubic types with rational double points which were already found by Schläfli and Cayley.

Example 1.33. Consider the first diagram $\Gamma$ of $N=7$ of AN04, Table 3] corresponding to quartic Del Pezzo surfaces: 


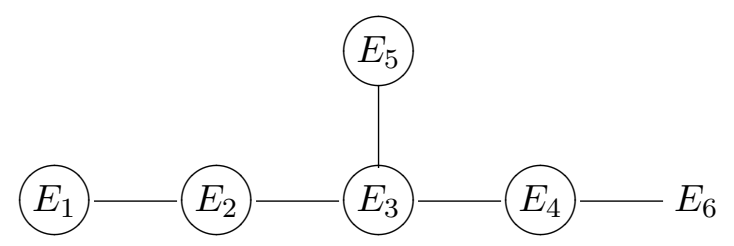

As before, we calculate the extended Dynkin diagrams for different choices of $(-2)$-curves $\Delta \subset \Delta_{0}:=\left\{E_{1}, \ldots, E_{5}\right\}$. The results can be found in Table 1.3 .

\begin{tabular}{|c|c|c|}
\hline$\Delta$ & singularity type & $\#\{(-1)$-curves $\}$ \\
\hline \hline$\left\{E_{1}, E_{2}, E_{3}\right\}$ & $\mathbf{A}_{3}$ & 5 \\
$\left\{E_{3}, E_{4}, E_{5}\right\}$ & $\mathbf{A}_{3}$ & 4 \\
\hline$\left\{E_{1}, E_{3}\right\}$ & $2 \mathbf{A}_{1}$ & 9 \\
$\left\{E_{4}, E_{5}\right\}$ & $2 \mathbf{A}_{1}$ & 8 \\
\hline
\end{tabular}

TABle 1.3. Quartic Del Pezzo surfaces

This shows that there are pairs of Del Pezzo surfaces of the same degree with the same singularity type which have different extended Dynkin diagrams. By BW79 and [CT88, the only other examples of this phenomenon in degree $\geq 3$ are surfaces in degree 6 of singularity type $\mathbf{A}_{1}$ with four or five lines.

Remark 1.34. Example 1.33 shows that two Del Pezzo surfaces may have different types, but the same singularity type.

However, if the type of a singular Del Pezzo surface is determined by its singularity type, we will use the same notation for both (e.g., "a singular cubic Del Pezzo surface of type $\mathbf{E}_{6}$ "). In the three cases of Example 1.33 , we will resolve the ambiguity by mentioning additionally the number of lines in the anticanonical embedding (e.g., "a singular quartic Del Pezzo surface of type $\mathbf{A}_{3}$ with five lines").

\subsection{Contracting (-1)-curves}

Let $S$ be a generalized Del Pezzo surface. By Theorem 1.25, the surface $S$ is $\mathbb{P}^{2}, \mathbb{P}^{1} \times \mathbb{P}^{1}, F_{2}$, or the blow-up of $\mathbb{P}^{2}$ in $r$ points in almost general position. In the latter case, how can we derive a configuration of points $p_{1}, \ldots, p_{r}$ in almost general position such that the blow-up $\pi: \widetilde{S}_{r} \rightarrow \mathbb{P}^{2}$ in $p_{1}, \ldots, p_{r}$ has a given extended Dynkin diagram $\Gamma\left(\widetilde{S}_{r}\right)$ ?

This question is interesting as we may have produced $\Gamma\left(\widetilde{S}_{r}\right)$ using the procedure described in Section 1.6, which does not involve information on the position of the blown-up points.

LEMMA 1.35. Let $\widetilde{S}_{r}$ be the blow-up of $\mathbb{P}^{2}$ in $r$ points in almost general position, and let $E_{r}$ be a (-1)-curve on $\widetilde{S}_{r}$. Let $\pi_{r}: \widetilde{S}_{r} \rightarrow \widetilde{S}_{r-1}$ be the contraction of $E_{r}$ to a point $p_{r} \in \widetilde{S}_{r-1}$. Then $\widetilde{S}_{r}$ is the blow-up of $\widetilde{S}_{r-1}$ in $p_{r}$, and $\widetilde{S}_{r-1}$ is a generalized Del Pezzo surface. 
Proof. As $\widetilde{S}_{r}$ is a blow-up of a smooth surface, there is at least one $(-1)$-curve on $\widetilde{S}_{r}$. By Castelnuovo's criterion [Har77, Theorem V.5.7], the contraction of a $(-1)$-curve $E$ on $\widetilde{S}_{r}$ to a point $p_{r}$ results in a smooth surface $\widetilde{S}_{r-1}$ such that $\widetilde{S}_{r}$ is the blow-up of $\widetilde{S}_{r-1}$ in $p_{r}$. By [DP80, Section III.9], $\widetilde{S}_{r-1}$ is a generalized Del Pezzo surface. (Note that the Hirzebruch surface $F_{2}$ was missed in [DP80, Section III.9], as mentioned after [CT88, Proposition 0.4].)

In this situation, we can extract the intersection numbers (including self intersection numbers) of the projections of the negative curves on $\widetilde{S}_{r}$ under $\pi_{r}$. The only change is that the projections of curves which intersect $E_{r}$ on $\widetilde{S}_{r}$ intersect pairwise on $\widetilde{S}_{r-1}$ and have a higher self intersection number (Lemma 1.2). Every negative curve on $\widetilde{S}_{r-1}$ is the projection of a negative curve on $\widetilde{S}_{r}$. This allows us to derive the extended Dynkin diagram of $\widetilde{S}_{r-1}$. Furthermore, we learn which projections of the negative curves pass through $p_{r}$, giving some information on the position of the blown-up point.

The degree of $\widetilde{S}_{r-1}$ is $9-(r-1)$. If this is $\leq 7$, i.e., $r \geq 3$, then $\widetilde{S}_{r-1}$ is the blow-up of $\mathbb{P}^{2}$ in $r-1$ points in almost general position. Therefore, any choice of $(-1)$-curve $E$ is suitable for a construction of $\widetilde{S}_{r}$ as the blow-up of $\mathbb{P}^{2}$ in $r$ points in almost general position.

On the other hand, if $r=2$, then $\Gamma\left(\widetilde{S}_{2}\right)$ is one of the following two diagrams:

- $\Gamma\left(\widetilde{S}_{2,1}\right)$ of a smooth Del Pezzo surface $S_{2}=\widetilde{S}_{2,1}$ of degree 7 :

$$
E_{1}-E_{2}-E_{3}
$$

- $\Gamma\left(\widetilde{S}_{2,2}\right)$ of a Del Pezzo surface $\widetilde{S}_{2,2}$ of degree 7 and type $\mathbf{A}_{1}$ :

$$
\text { (E) }-E_{2}-E_{3}
$$

Here, we must be careful with our choice of $(-1)$-curve, as in degree 8 , only a smooth Del Pezzo surface $S_{1}$ containing one $(-1)$-curve is the blow-up of $\mathbb{P}^{2}$. We must avoid $\mathbb{P}^{1} \times \mathbb{P}^{1}$ (containing no negative curves) and the Hirzebruch surface $F_{2}$ (containing one (-2)-curve).

The correct choice is to contract $E_{1}$ or $E_{3}$ in case of $\Gamma\left(\widetilde{S}_{2,1}\right)$ (contracting $E_{2}$ gives $\left.\mathbb{P}^{1} \times \mathbb{P}^{1}\right)$, and $E_{2}$ in case of $\Gamma\left(\widetilde{S}_{2,2}\right)\left(E_{1}\right.$ is a $(-2)$-curve, and contracting $E_{3}$ gives $F_{2}$ ) to obtain $S_{1}$.

For $r=1$, there is exactly one $(-1)$-curve on $S_{1}$, which we contract to obtain $\pi=\pi_{1} \circ \cdots \circ \pi_{r}: \widetilde{S}_{r} \underset{\widetilde{S}_{r}}{\rightarrow} \mathbb{P}^{2}$.

If a negative curve $E$ on $\widetilde{S}_{r}$ is not contracted at any stage of this process, then $\pi(E)$ is a curve in $\mathbb{P}^{2}$ whose self intersection number is the square of its degree. Furthermore, we know the relative configuration of all $\pi(E)$ and the positions of the blown-up points $p_{i}$ relative to them. Of course, some of the blown-up points may lie on exceptional divisors of previous blow-ups.

Example 1.36. Consider the extended Dynkin diagram of the cubic surface $\widetilde{S}_{6}$ of type $2 \mathbf{A}_{2}$ as constructed in Example 1.32 with negative curves $E_{1}, \ldots, E_{11}$. For $i$ from 6 down to 1 , the contraction

$$
\pi_{i}: \widetilde{S}_{i} \rightarrow \widetilde{S}_{i-1}
$$


maps the $(-1)$-curve $E_{i}$ to the point $p_{i} \in \widetilde{S}_{i-1}$, and the transform on $\widetilde{S}_{i-1}$ of a curve $E_{j}$ on $\widetilde{S}_{i}$ is also called $E_{j}$. We end up with $\widetilde{S}_{0}=\mathbb{P}^{2}$. Let $\pi:=\pi_{1} \circ \cdots \circ \pi_{6}$. In more detail, this works as follows:

- Contracting $E_{6}$ on $\widetilde{S}_{6}$ results in $\widetilde{S}_{5}$. The $(-2)$-curve $E_{5}$ on $\widetilde{S}_{6}$ turns into a $(-1)$-curve on $\widetilde{S}_{5}$ and intersects $E_{7}, \ldots, E_{10}$. The curve $E_{7}$ on $\widetilde{S}_{5}$ has self intersection number 0 .

- Contracting $E_{5}$ on $\widetilde{S}_{5}$ gives $\widetilde{S}_{4}$ on which $E_{7}, \ldots, E_{10}$ intersect in one point.

- Contracting $E_{i}$ on $\widetilde{S}_{i}$ for $i=4,3,2,1$ results in $\widetilde{S}_{0}=\mathbb{P}^{2}$ containing the curves $E_{7}, \ldots, E_{11}$. Their self intersection numbers have changed to 1 , so they are lines in $\mathbb{P}^{2}$. While $E_{7}, \ldots, E_{10}$ intersect in one point $p \in \mathbb{P}^{2}$, they are intersected by $E_{11}$ away from $p$.

Reversing this process, the blown-up points $p_{1}, \ldots, p_{6}$ are as follows:

$$
\begin{aligned}
& p_{1}=E_{10} \cap E_{11}, \quad p_{2}=E_{1} \cap E_{10}, \quad p_{3}=E_{8} \cap E_{11}, \\
& p_{4}=E_{9} \cap E_{11}, \quad p_{5}=E_{7} \cap E_{8} \cap E_{9} \cap E_{10}, \quad p_{6}=E_{5} \cap E_{7} .
\end{aligned}
$$

In terms of the standard basis (Lemma 1.7), $E_{1}, \ldots, E_{11}$ are:

$$
\begin{aligned}
E_{1} & =l_{1}-l_{2}, \quad E_{2}=l_{2}, \quad E_{3}=l_{3}, \quad E_{4}=l_{4}, \quad E_{5}=l_{5}-l_{6}, \\
E_{6} & =l_{6}, \quad E_{7}=l_{0}-l_{5}-l_{6}, \quad E_{8}=l_{0}-l_{3}-l_{5}, \quad E_{9}=l_{0}-l_{4}-l_{5}, \\
E_{10} & =l_{0}-l_{1}-l_{2}-l_{5}, \quad E_{11}=l_{0}-l_{1}-l_{3}-l_{4} .
\end{aligned}
$$

The surface $\widetilde{S}_{6}$ is determined by the position of the lines $E_{7}, \ldots, E_{11}$ in $\mathbb{P}^{2}$. Using automorphisms of $\mathbb{P}^{2}$ as in Remark 1.17, we may assume that $p=(1: 0: 0)$ in $\mathbb{P}^{2}=\left\{\left(x_{0}: x_{1}: x_{2}\right)\right\}$, while $E_{11}=\left\{x_{0}=0\right\}$. Furthermore, we may assume that three of the four intersection points of $E_{11}$ with $E_{7}, \ldots, E_{10}$ are at certain positions, while the choice of the fourth results in a one-parameter family of generalized Del Pezzo surfaces of type $2 \mathbf{A}_{2}$ :

$$
\begin{aligned}
& E_{7}=\left\{x_{1}=0\right\}, \quad E_{8}=\left\{x_{2}=0\right\}, \\
& E_{9}=\left\{x_{1}-x_{2}=0\right\}, \quad E_{10}=\left\{x_{1}-\alpha x_{2}=0\right\} .
\end{aligned}
$$

The parameter $\alpha$ can take any value in $\mathbb{K} \backslash\{0,1\}$. In Section 6.6, we will return to this family of surfaces.

This example shows that there may be an infinite family of isomorphy classes of cubic Del Pezzo surfaces of the same type. In other cases of degree 3 , the number of isomorphy classes of a given type is finite:

- Type $\mathbf{E}_{6}$ : Exactly one isomorphy class of cubic surfaces exists (see Example 1.27).

- Type $\mathbf{D}_{4}$ : Exactly two isomorphy classes exist (see [HT04, Remark 4.1]).

For singular cubic Del Pezzo surfaces, this and the number of parameters in each infinite family can be found in [BW79].

\subsection{Toric Del Pezzo surfaces}

For an introduction to toric varieties, see [Ful93. Toric surfaces are equivariant compactifications of a 2-dimensional torus $T$, for example $\mathbb{P}^{2}$, $\mathbb{P}^{1} \times \mathbb{P}^{1}$, and the Hirzebruch surface $F_{2}$. 
A toric surface contains divisors which are invariant under the action of $T$. The $T$-invariant prime divisors intersect exactly in points which are fixed under $T$. In case of $\mathbb{P}^{2}$, the $T$-invariant prime divisors are three lines forming a triangle.

The blow-up of a toric variety is again toric if and only if we blow up a point which is fixed under the action of $T$, i.e., an intersection point of two $T$-invariant prime divisors $D_{1}, D_{2}$. The exceptional divisor $E$ of the blow-up is $T$-invariant. Fixed points on $E$ are exactly the intersection points of $E$ with $D_{1}$ and $D_{2}$.

To construct toric generalised Del Pezzo surfaces other than $\mathbb{P}^{1} \times \mathbb{P}^{1}$ and $F_{2}$, we blow up $\mathbb{P}^{2}$ in this way. Note that the only negative curves are exceptional divisors and possibly the strict transforms of the three $T$ invariant lines on $\mathbb{P}^{2}$, i.e., a subset of all $T$-invariant prime divisors.

If we include all $T$-invariant prime divisors in the extended Dynkin diagram, it has the shape of a "circle". We denote it as a vector of self intersection numbers. Two entries are next two each other (where we consider the first and last entry as "next to each other") if the corresponding prime divisors intersect. See Table 1.4 for the extended Dynkin diagrams of all toric generalized Del Pezzo surfaces.

This result allows us to identify non-toric Del Pezzo surfaces from their extended Dynkin diagrams: Whenever a negative curve intersects more than two other negative curves, the surface cannot be toric.

\begin{tabular}{|c|c|c|}
\hline degree & type & extended Dynkin diagram \\
\hline 9 & $\mathbb{P}^{2}$ & $(1,1,1)$ \\
\hline 8 & $\begin{array}{c}\text { smooth } S_{1} \\
\mathbb{P}^{1} \times \mathbb{P}^{1} \\
F_{2}\end{array}$ & $\begin{array}{c}(1,0,-1,0) \\
(0,0,0,0) \\
(-2,0,2,0)\end{array}$ \\
\hline 7 & $\begin{array}{c}\text { smooth } S_{2} \\
\mathbf{A}_{1}\end{array}$ & $\begin{array}{l}(0,-1,-1,-1,0) \\
(1,0,-2,-1,-1)\end{array}$ \\
\hline 6 & $\begin{array}{c}\text { smooth } S_{3} \\
\mathbf{A}_{1}(4 \text { lines }) \\
2 \mathbf{A}_{1} \\
\mathbf{A}_{2}+\mathbf{A}_{1}\end{array}$ & $\begin{array}{c}(-1,-1,-1,-1,-1,-1) \\
(0,-1,-1,-2,-1,-1) \\
(0,-2,-1,-2,-1,0) \\
(1,0,-2,-2,-1,-2)\end{array}$ \\
\hline 5 & $\begin{array}{c}2 \mathbf{A}_{1} \\
\mathbf{A}_{2}+\mathbf{A}_{1}\end{array}$ & $\begin{array}{c}(-1,-1,-1,-1,-2,-1,-2) \\
(0,-1,-1,-2,-2,-1,-2)\end{array}$ \\
\hline 4 & $\begin{array}{c}4 \mathbf{A}_{1} \\
\mathbf{A}_{2}+2 \mathbf{A}_{1} \\
\mathbf{A}_{3}+2 \mathbf{A}_{1}\end{array}$ & $\begin{array}{c}(-1,-2,-1,-2,-1,-2,-1,-2) \\
(-2,-1,-2,-1,-1,-2,-1,-2) \\
(0,-2,-1,-2,-2,-2,-1,-2) \\
\end{array}$ \\
\hline 3 & $3 \mathbf{A}_{2}$ & $(-2,-2,-1,-2,-2,-1,-2,-2,-1)$ \\
\hline
\end{tabular}

TABle 1.4. Toric Del Pezzo surfaces. 



\section{CHAPTER 2}

\section{Universal torsors and Cox rings}

\subsection{Introduction}

Universal torsors were introduced by Colliot-Thélène and Sansuc in connection with their studies of the Hasse principle for Del Pezzo surfaces of degrees 3 and 4 [CTS80, [CTS87]. We will see in Part 2 how they can be applied to Manin's conjecture.

Over an algebraically closed field $\mathbb{K}$ of characteristic 0 , a universal torsor of a generalized Del Pezzo surface $S_{r}$ of degree $9-r$ is constructed as follows: Let $\mathcal{L}_{0}, \ldots, \mathcal{L}_{r}$ be invertible sheaves whose classes form a basis of $\operatorname{Pic}\left(S_{r}\right)$. Let $\mathcal{L}_{i}^{\circ}$ be the sheaf obtained by removing the zero section from $\mathcal{L}_{i}$. Then the bundle

$$
\mathcal{T}_{S_{r}}:=\mathcal{L}_{0}^{\circ} \times_{S_{r}} \cdots \times_{S_{r}} \mathcal{L}_{r}^{\circ}
$$

over $S_{r}$ is a universal torsor (see Lemma 2.3).

The Cox ring, or homogeneous coordinate ring, of $S_{r}$ is the space

$$
\operatorname{Cox}\left(S_{r}\right)=\bigoplus_{\left(\nu_{0}, \ldots, \nu_{r}\right) \in \mathbb{Z}^{r+1}} H^{0}\left(S_{r}, \mathcal{L}_{0}^{\otimes \nu_{0}} \otimes \cdots \otimes \mathcal{L}_{r}^{\otimes \nu_{r}}\right)
$$

whose ring structure is induced by the multiplication of global sections. We will see that $\mathcal{T}_{S_{r}}$ is an open subset of $\mathbb{A}\left(S_{r}\right):=\operatorname{Spec}\left(\operatorname{Cox}\left(S_{r}\right)\right)$.

This section has the following structure: In Section 2.2, we discuss universal torsors over non-closed fields. In Section 2.3, we give basic properties of Cox rings. In Section 2.4, we collect some preliminary results on generators of a Cox ring as a $\mathbb{K}$-algebra and relations between these generators.

The following Chapters 3, 5, and 6 are concerned with the explicit determination of generators and relations in Cox rings of generalized Del Pezzo surfaces.

\subsection{Universal torsors}

Over a field $\mathbb{K}$ of characteristic 0 with algebraic closure $\overline{\mathbb{K}}$, let $S$ be a smooth projective surface, with geometric Picard $\operatorname{group} \operatorname{Pic}\left(S_{\overline{\mathbb{K}}}\right) \cong \mathbb{Z}^{r+1}$, where $S_{\overline{\mathbb{K}}}:=S \times_{\text {Spec } \mathbb{K}}$ Spec $\overline{\mathbb{K}}$.

Let $T$ be an algebraic torus, i.e., a linear algebraic group such that $T_{\overline{\mathbb{K}}}$ is isomorphic to $\mathbb{G}_{\mathrm{m}}^{s}$ for some $s \in \mathbb{Z}_{>0}$. Let $\mathcal{T}$ be a variety with a faithfully flat morphism $\pi: \mathcal{T} \rightarrow S$ and an action of $T$ on $\mathcal{T}$. As explained in Pey98, Section 3.3], $\mathcal{T}$ is called a $T$-torsor over $S$ if and only if the natural map $T \times{ }_{\text {Spec } \mathbb{K}} \mathcal{T} \rightarrow \mathcal{T} \times{ }_{S} \mathcal{T}$ is an isomorphism.

The $T$-torsors over $S$ up to isomorphism are classified by the étale cohomology group $H_{\text {ét }}^{1}(S, T)$. 
Proposition 2.1. For every $p \in S(\mathbb{K})$, there is a $T$-torsor $\pi_{p}: \mathcal{T}_{p} \rightarrow S$ (unique up to isomorphism) such that $p \in \pi_{p}\left(\mathcal{T}_{p}(\mathbb{K})\right)$.

If $\mathbb{K}$ is a number field, $S(\mathbb{K})$ is the union of $\pi_{p_{i}}\left(\mathcal{T}_{p_{i}}(\mathbb{K})\right)$ for a finite set of points $p_{1}, \ldots, p_{n} \in S(\mathbb{K})$.

Proof. We construct $\pi_{p}: \mathcal{T}_{p} \rightarrow S$ as follows (see [CTS80, Section II]): We have a map

$$
\begin{array}{ccc}
S(\mathbb{K}) \times H_{\text {ét }}^{1}(S, T) & \rightarrow & H^{1}(\mathbb{K}, T) \\
(p,[\mathcal{T}]) & \mapsto & {[\mathcal{T}(p)],}
\end{array}
$$

where $\mathcal{T}(p):=\mathcal{T} \times_{S} \operatorname{Spec} \mathbb{K}(p)$ is a $\mathbb{K}$-form of $T$, with $[\mathcal{T}(p)]=0$ in the Galois cohomology group

$$
H^{1}(\mathbb{K}, T)=H^{1}(\operatorname{Gal}(\overline{\mathbb{K}} / \mathbb{K}), T(\overline{\mathbb{K}}))
$$

if and only if $p \in \pi(\mathcal{T}(\mathbb{K}))$. Using the map $\phi: H^{1}(\mathbb{K}, T) \rightarrow H_{\text {ét }}^{1}(S, T)$, we can construct a torsor $\pi_{p}: \mathcal{T}_{p} \rightarrow S$ of the class $[\mathcal{T}]-\phi([\mathcal{T}(p)]) \in H_{\text {ét }}^{1}(S, T)$ such that $p \in \pi_{p}\left(\mathcal{T}_{p}\right)$.

See [CTS80, Proposition 2] for the second statement.

The Picard group $\operatorname{Pic}\left(S_{\overline{\mathbb{K}}}\right)$ and the group of characters

$$
\mathfrak{X}^{*}(T):=\operatorname{Hom}\left(T_{\overline{\mathbb{K}}}, \mathbb{G}_{\mathrm{m}}\right)
$$

of the torus $T$ are free $\mathbb{Z}$-modules with an action of $\mathrm{Gal}(\overline{\mathbb{K}} / \mathbb{K})$. By [CTS87, Section 2.2], there is a map

$$
\rho: H_{\text {ét }}^{1}(S, T) \rightarrow \operatorname{Hom}_{\mathrm{Gal}(\overline{\mathbb{K}} / \mathbb{K})}\left(\mathfrak{X}^{*}(T), \operatorname{Pic}\left(S_{\overline{\mathbb{K}}}\right)\right),
$$

defined by $\rho([\mathcal{T}])(\chi):=\chi_{*}(\mathcal{T})$ for any $\chi \in \mathfrak{X}^{*}(T)$. Here, $\operatorname{Hom}_{\mathrm{Gal}(\overline{\mathbb{K}} / \mathbb{K})}(\cdot, \cdot)$ denotes the homomorphisms of free $\mathbb{Z}$-modules which are compatible with the $\operatorname{Gal}(\overline{\mathbb{K}} / \mathbb{K})$-action.

The torus $T_{\mathrm{NS}}(S):=\operatorname{Hom}\left(\operatorname{Pic}(S), \mathbb{G}_{\mathrm{m}}\right)$ is called the Néron-Severi torus of $S$. If the ground field $\mathbb{K}$ is algebraically closed, it is isomorphic to $\mathbb{G}_{\mathrm{m}}^{r+1}$ after the choice of a basis of $\operatorname{Pic}(S)$. The group of characters $\mathfrak{X}^{*}\left(T_{\mathrm{NS}}(S)\right)$ is canonically isomorphic to $\operatorname{Pic}\left(S_{\overline{\mathbb{K}}}\right)$.

Definition 2.2. A universal torsor $\mathcal{T}_{S}$ over $S$ as above is a $T_{\mathrm{NS}}(S)$ torsor such that $\rho\left(\left[\mathcal{T}_{S}\right]\right)=\operatorname{id} \operatorname{Pic}\left(S_{\overline{\mathbb{K}}}\right)$.

LEMma 2.3. Let $\mathbb{K}$ be an algebraically closed field of characteristic 0. Let $\mathcal{L}_{0}, \ldots, \mathcal{L}_{r}$ be a basis of $\operatorname{Pic}(S)$. The bundle

$$
\mathcal{T}:=\mathcal{L}_{0}^{\circ} \times{ }_{S} \cdots \times{ }_{S} \mathcal{L}_{r}^{\circ}
$$

is a universal torsor over $S$.

Proof. The isomorphism $\phi: \operatorname{Pic}\left(S_{\overline{\mathbb{K}}}\right) \rightarrow \mathfrak{X}^{*}\left(T_{\mathrm{NS}}(S)\right)$ gives a basis $\chi_{0}, \ldots, \chi_{r}$ of $\mathfrak{X}^{*}\left(T_{\mathrm{NS}}(S)\right)$, with $\chi_{i}:=\phi\left(\mathcal{L}_{i}\right)$. By definition, $\rho([\mathcal{T}])\left(\chi_{i}\right)$ for the character $\chi_{i} \in \mathfrak{X}^{*}\left(T_{\mathrm{NS}}(S)\right)$ is the class of $\left(\chi_{i}\right)_{*}(\mathcal{T})$ in $\operatorname{Pic}\left(S_{\overline{\mathbb{K}}}\right)$, which is $\mathcal{L}_{i}$ as required.

Over an algebraically closed field of characteristic $0, \mathcal{T}_{S}$ as defined in Lemma 2.3 does not depend on the chosen basis of Pic $(S)$ by [Pey04, Proposition 8]. In fact, every universal torsor is isomorphic to it.

Over non-closed fields $\mathbb{K}$, the existence of a $\mathbb{K}$-rational point $p$ on $S$ implies the existence of a universal torsor $\pi: \mathcal{T}_{S} \rightarrow S$, defined over $\mathbb{K}$ 
(Proposition 2.1). In this case, $\mathcal{T}_{S}$ becomes isomorphic over $\overline{\mathbb{K}}$ to $\mathcal{T}$ as in Lemma 2.3 . If $S(\mathbb{K})=\emptyset$, a universal torsor does not necessarily exist over $\mathbb{K}$.

\subsection{Cox rings}

The following construction is due to [HK00, generalizing the homogeneous coordinate ring of toric varieties in Cox95.

As before, let $\mathcal{L}_{0}, \ldots, \mathcal{L}_{r}$ be a basis of $\operatorname{Pic}(S) \cong \mathbb{Z}^{r+1}$ for a smooth projective surface $S$ over an algebraically closed field $\mathbb{K}$. Let $H^{0}(S, \mathcal{L})$ be the $\mathbb{K}$-vector space of global sections of $\mathcal{L} \in \operatorname{Pic}(S)$, which we also denote by $H^{0}(\mathcal{L})$.

For $\boldsymbol{\nu}=\left(\nu_{0}, \ldots, \nu_{r}\right) \in \mathbb{Z}^{r+1}$, let

$$
\mathcal{L}^{\nu}:=\mathcal{L}_{0}^{\otimes \nu_{0}} \otimes \cdots \otimes \mathcal{L}_{r}^{\otimes \nu_{r}} .
$$

For $\boldsymbol{\nu}, \boldsymbol{\mu} \in \mathbb{Z}^{r+1}$, the multiplication of sections defines a map

$$
H^{0}\left(\mathcal{L}^{\boldsymbol{\nu}}\right) \times H^{0}\left(\mathcal{L}^{\boldsymbol{\mu}}\right) \rightarrow H^{0}\left(\mathcal{L}^{\boldsymbol{\nu}+\boldsymbol{\mu}}\right) .
$$

Definition 2.4. For $S$ and $\mathcal{L}_{0}, \ldots, \mathcal{L}_{r}$ as above, the Cox ring, or homogeneous coordinate ring, of $S$ is defined as

$$
\operatorname{Cox}(S):=\bigoplus_{\boldsymbol{\nu} \in \mathbb{Z}^{r+1}} H^{0}\left(\mathcal{L}^{\boldsymbol{\nu}}\right)
$$

where the multiplication of sections

$$
H^{0}\left(\mathcal{L}^{\nu}\right) \times H^{0}\left(\mathcal{L}^{\boldsymbol{\mu}}\right) \rightarrow H^{0}\left(\mathcal{L}^{\boldsymbol{\nu}+\boldsymbol{\mu}}\right)
$$

induces the multiplication in $\operatorname{Cox}(S)$.

For any algebra $A$ with a $\operatorname{Pic}(S)$-grading, we will denote the part of degree $\mathcal{O}(D)$ for a divisor $D$ by $A_{D}$ or $A_{\mathcal{O}(D)}$. We have a $\operatorname{Pic}(S)$-grading on $\operatorname{Cox}(S)$ as follows:

$$
\operatorname{Cox}(S)_{D}=\operatorname{Cox}(S)_{\mathcal{O}(D)}=H^{0}(\mathcal{O}(D)) .
$$

If $\mathcal{L}^{\nu} \preceq \mathcal{L}^{\mu}$ in the partial ordering of $\operatorname{Pic}(S)$, multiplication by a non-zero global section of $\mathcal{L}^{\boldsymbol{\mu}-\boldsymbol{\nu}}$ induces an inclusion

$$
\operatorname{Cox}(S)_{\mathcal{L}^{\nu}} \hookrightarrow \operatorname{Cox}(S)_{\mathcal{L}^{\mu}} .
$$

As remarked in [HK00, Section 2], the Cox ring of $S$ is unique up to isomorphism. However, we cannot simply define it without the choice of a basis of $\operatorname{Pic}(S)$ since the multiplication would be defined only up to a constant.

\subsection{Generators and relations}

Let $S$ be a generalized Del Pezzo surface over an algebraically closed field $\mathbb{K}$ of characteristic 0 . A line bundle $\mathcal{L} \in \operatorname{Pic}(S)$ has global sections if and only if $\mathcal{L}$ is the class of an effective divisor. Therefore, the $\operatorname{Pic}(S)$-degrees in which $\operatorname{Cox}(S)$ is non-zero lie in the effective cone $\Lambda_{\text {eff }}(S)$ (Definition 1.8).

Lemma 2.5. The ring $\operatorname{Cox}(S)$ is a finitely generated $\mathbb{K}$-algebra. Let $N$ be the minimal number of generators of $\operatorname{Cox}(S)$.

- We can find a system of $N$ generators which are homogeneous with respect to the $\operatorname{Pic}(S)$-grading. 
- Up to permutation, the $\operatorname{Pic}(S)$-degrees of a minimal system of homogeneous generators are unique.

- Given any set of homogeneous generators, we can find a subset of $N$ elements which is a generating system.

Proof. The Cox ring is finitely generated by HK00, Corollary 2.16 and Proposition 2.9]. Note that we will also prove this in Theorem 6.2 if the degree of $S$ is $\geq 2$. Since $\mathbb{K}$ is algebraically closed, $\operatorname{Cox}(S)$ is by definition the direct sum of its homogeneous components. Hence, we can find a minimal system of generators containing only homogeneous elements.

We use the partial order on the effective divisor classes. Any homogeneous expression involving an element of degree $\mathcal{L}^{\prime} \in \operatorname{Pic}(S)$ has degree $\mathcal{L}$ with $\mathcal{L}^{\prime} \preceq \mathcal{L}$. Consequently, $\operatorname{Cox}(S)_{\mathcal{L}}$ is generated by elements of degree $\mathcal{L}^{\prime} \preceq \mathcal{L}$

Let $C$ be the subalgebra of $\operatorname{Cox}(S)$ generated by all elements of degree $\mathcal{L}^{\prime} \prec \mathcal{L}$. For a system of homogeneous generators of $\operatorname{Cox}(S)$, the generators of degree $\mathcal{L}^{\prime} \prec \mathcal{L}$ generate exactly $C$ since generators of degree $\mathcal{L}^{\prime \prime} \nprec \mathcal{L}$ cannot affect the degrees $\mathcal{L}^{\prime} \prec \mathcal{L}$. Therefore, we have at least

$$
n_{\mathcal{L}}:=\operatorname{dim}\left(\operatorname{Cox}(S)_{\mathcal{L}}\right)-\operatorname{dim}\left(C_{\mathcal{L}}\right)
$$

generators of degree $\mathcal{L}$.

Therefore, any system of homogeneous generators must contain at least $n_{\mathcal{L}}$ elements of degree $\mathcal{L}$. If there are more than that for some $\mathcal{L}$, we can remove an appropriate number of generators of degree $\mathcal{L}$ since we are simply looking for a basis in the vector space $\operatorname{Cox}(S)_{\mathcal{L}}$.

REMARK 2.6. Once any set of generators is known, we can find a minimal generating set: First, we replace each generator by its homogeneous parts. Then we go through the degrees $\mathcal{L}$ of these homogeneous generators in their partial ordering and check for each $\mathcal{L}$ whether we may remove some of the generators, as explained in the proof of Lemma 2.5 .

We will see in the following chapters how to determine systems of generators.

Lemma 2.7. If $E$ is a negative curve on $S$, then every homogeneous system of generators of $\operatorname{Cox}(S)$ contains a section of degree $E$.

The number of generators of $\operatorname{Cox}(S)$ must be at least the number of negative curves.

Proof. By Lemma 1.3, the space $H^{0}(\mathcal{O}(E))$ is one-dimensional.

If a non-zero global section $s$ of $\mathcal{O}(E)$ can be expressed in terms of homogeneous sections of other degrees, then $\mathcal{O}(E)$ must be a non-trivial non-negative linear combination of other effective divisor classes. This would allow us to construct a section which is linearly independent of $s$.

However, the example of the $\mathbf{E}_{6}$ cubic surface [HT04, Section 3] shows that the Cox ring of a generalized Del Pezzo surface $S$ can have other generators besides sections of negative curves. In view of Lemma 1.15 and Proposition 8.11, the following result shows that the degrees of these generators lie in the nef cone if the degree of $S$ is $\leq 7$. 
Lemma 2.8. Suppose $\alpha \in H^{0}(A)$ an element of a miminal system of homogeneous generators of $\operatorname{Cox}(S)$. Then $A$ is a negative curve, or $(A, E) \geq$ 0 for all negative curves $E$ on $S$.

Proof. Suppose $\alpha \in H^{0}(A)$, and $\left(A, E_{i}\right)<0$ for some negative curves $E_{i}$ with $i \in\{1, \ldots, s\}$. Then $E_{1}, \ldots, E_{s}$ are fixed components of $A$, with multiplicities $e_{1}, \ldots, e_{s}$. We can write $A=B+\sum_{i=1}^{s} e_{i} E_{i}$, where $(B, E) \geq 0$ for all negative curves $E$. Multiplication by $\eta_{1}^{e_{1}} \cdots \eta_{s}^{e_{s}}$ gives an isomorphism

$$
H^{0}(B) \rightarrow H^{0}(A)
$$

where $\eta_{i}$ is a non-zero section of $H^{0}\left(E_{i}\right)$. Therefore, we have $\alpha=\beta \cdot \eta_{1}^{e_{1}} \cdots \eta_{s}^{e_{s}}$ for some $\beta \in H^{0}(B)$.

Let

$$
\mathbb{A}(S):=\operatorname{Spec}(\operatorname{Cox}(S)),
$$

which is of finite type by Lemma 2.5. Having determined a finite set of homogeneous generators $\xi_{1}, \ldots, \xi_{N}$, i.e., $\operatorname{Cox}(S)=\mathbb{K}\left[\xi_{1}, \ldots, \xi_{N}\right] / I$ for an ideal $I$ of relations, we can regard $\mathbb{A}(S)$ as embedded in affine space

$$
\mathbb{A}^{N}=\operatorname{Spec}\left(\mathbb{K}\left[\xi_{1}, \ldots, \xi_{N}\right]\right) .
$$

If a minimal system of homogeneous expressions generating $I$ has $n$ elements, we say that $\operatorname{Cox}(S)$ has $N$ generators and $n$ relations.

Lemma 2.9. The variety $\mathbb{A}(S)$ is irreducible.

Proof. We must show that $\operatorname{Cox}(S)$ is an integral domain. Since $S$ is irreducible, this follows from [EKW04, Corollary 1.2].

Proposition 2.10. The universal torsor $\mathcal{T}_{S}$ is an open subset of $\mathbb{A}(S)$.

Proof. See [HK00, Corollary 2.16 and Proposition 2.9].

REMARK 2.11. The Néron-Severi torus $T_{\mathrm{NS}}(S)$ acts on $\mathbb{A}(S)$ : The action of $T_{\mathrm{NS}}(S)$ on each coordinate $\xi_{i}$ of $\mathbb{A}^{N}=\operatorname{Spec}\left(\mathbb{K}\left[\xi_{1}, \ldots, \xi_{N}\right]\right)$ is given by the character $\chi_{i}$ corresponding to the degree of $\xi_{i}$ in $\operatorname{Pic}(S) \cong \mathfrak{X}^{*}\left(T_{\mathrm{NS}}(S)\right)$. As the ideal $I$ of relations in $\operatorname{Cox}(S)$, which defines $\mathbb{A}(S)$ as a subvariety of $\mathbb{A}^{N}$, is generated by polynomials in $\mathbb{K}\left[\xi_{1}, \ldots, \xi_{N}\right]$ which are homogeneous with respect to the $\operatorname{Pic}(S)$-grading, the action of $T_{\mathrm{NS}}(S)$ on $\mathbb{A}^{N}$ induces an action on $\mathbb{A}(S)$. By the constructions of [HK00], this action extends the natural action of $T_{\mathrm{NS}}(S)$ on $\mathcal{T}_{S} \subset \mathbb{A}(S)$.

By the next lemma, there is no relation between generators of the Cox ring in case of toric Del Pezzo surfaces (Section 1.8). The degrees in $\operatorname{Pic}(S)$ of the generators can be extracted from the extended Dynkin diagrams in Table 1.4.

Lemma 2.12. A generalized Del Pezzo surface $S$ is toric if and only if its Cox ring is a polynomial ring.

In this case, generators of $\operatorname{Cox}(S)$ are sections corresponding to prime divisors which are invariant under the action of the open torus in $S$.

Proof. This follows from [Cox95. See [HK00, Corollary 2.10].

Distinguishing between the following three types of Cox rings and universal torsors will be important in Chapter 5 . 
Lemma 2.13. Let $S$ be a generalized Del Pezzo surface of degree $9-r$, with universal torsor $\mathcal{T}_{S}$. Let $N$ be the minimal number of generators of $\operatorname{Cox}(S) \cong \mathbb{K}\left[\xi_{1}, \ldots, \xi_{N}\right] / I$, where $I$ is the ideal of relations between these generators.

- It is an open subset of affine space if and only if $N=r+3$, and $I$ is trivial.

- It is an open subset of a hypersurface if and only if $N=r+4$, and I has one generator.

- It has codimension $\geq 2$ in $\mathbb{A}^{N}$ if and only if $N \geq r+5$, and $I$ has at least two independent generators.

Proof. For the dimension of the universal torsor, we have

$$
\operatorname{dim}\left(\mathcal{T}_{S}\right)=\operatorname{dim}(S)+\operatorname{dim}\left(T_{\mathrm{NS}}(S)\right)=r+3
$$

As the universal torsor is an open subset of $\mathbb{A}(S)$ by Proposition 2.10, $\operatorname{Cox}(S)$ is a free polynomial ring with $r+3$ generators, or it has $r+4$ generators whose ideal of relations is generated by one equation, or at least $r+5$ generators and at least two independent relations. 


\section{CHAPTER 3}

\section{Cox rings of smooth Del Pezzo surfaces}

\subsection{Introduction}

We determine the Cox ring (Definition 2.4) of smooth Del Pezzo surfaces $S_{r}$ (cf. Section 1.3) whose degree $9-r$ is at most 5 . The results appeared in [Der06b].

The structure of the Cox ring of smooth Del Pezzo surfaces of degree $\geq 6$ is known from Lemma 2.12 since these surfaces are toric (see Table 1.4).

For $r \in\{3, \ldots, 7\}$, $\operatorname{Cox}\left(S_{r}\right)$ is generated by non-zero sections of the $N_{r}$ $(-1)$-curves ([BP04, Theorem 3.2]), see Table 3.1 for the values of $N_{r}$. For $r=8$, we must add two independent sections of $H^{0}\left(S_{8},-K_{S_{8}}\right)$. Let $R_{r}$ be the free polynomial ring whose variables correspond to these generators of $\operatorname{Cox}\left(S_{r}\right)$. We want to determine relations between these generators.

Definition 3.1. For $n \geq 1$, a divisor class $D$ is called an $(n)$-ruling if $D=D_{1}+D_{2}$ for two $(-1)$-curves $D_{1}, D_{2}$ whose intersection number $\left(D_{1}, D_{2}\right)$ is $n$. A (1)-ruling is also called a ruling.

By Lemma 3.3, each $(n)$-ruling defines quadratic relations between generators of $\operatorname{Cox}\left(S_{r}\right)$. Relations coming from (1)-rulings generate an ideal $I_{r} \subset R_{r}$. For $r \in\{4,5,6\}, \operatorname{Cox}\left(S_{r}\right)=R_{r} / \operatorname{rad}\left(I_{r}\right)$ by [BP04, Theorem 4.9]. We extend this result to $r \in\{7,8\}$ as follows: where

Theorem 3.2. For $r \in\{4, \ldots, 8\}$, we have $\operatorname{Cox}\left(S_{r}\right)=R_{r} / \operatorname{rad}\left(J_{r}\right)$,

- for $r \in\{4,5,6\}, J_{r}:=I_{r}$;

- the ideal $J_{7}$ is generated by the 504 quadratic relations coming from the 126 rulings, and 25 quadratic relations coming from the (2)ruling $-K_{S_{7}}$;

- the ideal $J_{8}$ is generated by the 10800 quadratic relations coming from the 2160 rulings, 6480 quadratic relations coming from 240 (2)-rulings, and 119 quadratic relations coming from the (3)-ruling $-2 K_{S_{8}}$.

After explaining some results on the relations in the Cox ring of smooth Del Pezzo surfaces in Section 3.2. we will discuss the cases $r \in\{6,7,8\}$ separately.

\subsection{Relations in the Cox ring}

Let $r \geq 3$. Recall the information from Section 1.3 on the $(-1)$-curves of $S_{r}$, whose number is $N_{r}$ as in Table 3.1. By Section 1.4, the Weyl group $W_{r}$ associated to the root system $\Phi_{r}$ as in Table 1.2 acts on $\operatorname{Pic}\left(S_{r}\right)$. 
For $r \leq 6$, the relations in the Cox ring are induced by rulings, and these relations also play an important role for $r \in\{7,8\}$. More precisely, by the discussion following [BP04, Remark 4.7], each ruling is represented in $r-1$ different ways as the sum of two $(-1)$-curves, giving $r-3$ linearly independent quadratic relations in $\operatorname{Cox}\left(S_{r}\right)$. Therefore, if each of the $N_{r}$ $(-1)$-curves intersects $n_{r}(-1)$-curves with intersection number 1 , we have $N_{r}^{\prime}=\left(N_{r} \cdot n_{r}\right) / 2$ pairs, the number of rulings is $N_{r}^{\prime \prime}=N_{r}^{\prime} /(r-1)$, and the number of quadratic relations coming from rulings is $N_{r}^{\prime \prime} \cdot(r-3)$ (see Table 3.1).

\begin{tabular}{|c||c|c|c|c|c|c|}
\hline$r$ & 3 & 4 & 5 & 6 & 7 & 8 \\
\hline \hline$N_{r}$ & 6 & 10 & 16 & 27 & 56 & 240 \\
$n_{r}$ & 2 & 3 & 5 & 10 & 27 & 126 \\
$N_{r}^{\prime \prime}$ & 3 & 5 & 10 & 27 & 126 & 2160 \\
\hline \hline relations & 0 & 5 & 20 & 81 & 504 & 10800 \\
\hline
\end{tabular}

TABLE 3.1. The number of relations coming from rulings.

Now we describe how to obtain explicit equations for $\operatorname{Cox}\left(S_{r}\right)$ and how to prove Theorem 3.2 . We isolate the steps that must be carried out for each of the degrees 3,2 , and 1 and complete the proofs in the following sections.

Choice of coordinates. Choose coordinates for $p_{1}, \ldots, p_{r} \in \mathbb{P}^{2}$. By Remark 1.17, we may assume that the first four points are

$$
p_{1}=(1: 0: 0), \quad p_{2}=(0: 1: 0), \quad p_{3}=(0: 0: 1), \quad p_{4}=(1: 1: 1),
$$

and we can write $p_{j}=\left(1: \alpha_{j}: \beta_{j}\right)$ for $j \in\{5, \ldots, r\}$.

Curves in $\mathbb{P}^{2}$. As explained in the introduction, $\operatorname{Cox}\left(S_{r}\right)$ is generated by sections of the $(-1)$-curves for $r \leq 7$. For a $(-1)$-curve $D$, we denote the corresponding section by $\xi(D)$, and for a generating section $\xi$, let $D(\xi)$ be the corresponding divisor. For $r=8$, we need two further generators: linearly independent sections $\kappa_{1}, \kappa_{2}$ of $-K_{S_{8}}$. Let $K_{1}:=D\left(\kappa_{1}\right), K_{2}:=D\left(\kappa_{2}\right)$ be the corresponding divisors in the divisor class $-K_{S_{8}}$.

Let $\mathcal{D}_{r}$ be the set of divisors corresponding to sections generating $\operatorname{Cox}\left(S_{r}\right)$ (including $K_{1}, K_{2}$ if $r=8$ ).

We need an explicit description of the image of each generator $D$ of $\operatorname{Cox}\left(S_{r}\right)$ under the projection $\pi: S_{r} \rightarrow \mathbb{P}^{2}$. According to Lemma 1.14, $\pi(D)$ can be a curve, determined by a form $f_{D}$ of degree $d \in\{1, \ldots, 6\}$, or a point (if $D=E_{i}$ ). If $\pi(D)$ is a point, the convention to choose $f_{D}$ as a non-zero constant will be useful later.

For $r=8$, we have the following situation: The image of $K_{i}$ is a cubic through the eight points $p_{1}, \ldots, p_{8}$. The choice of two linearly independent sections $\kappa_{1}, \kappa_{2}$ corresponds to the choice of two independent cubic forms $f_{K_{1}}, f_{K_{2}}$ vanishing in the eight points. Every cubic through these points has the form $a_{1} f_{K_{1}}+a_{2} f_{K_{2}}$ where $\left(a_{1}, a_{2}\right) \neq(0,0)$, and the cubic does not change if we replace $\left(a_{1}, a_{2}\right)$ be a non-zero multiple. This gives a one-dimensional projective space of cubics through the eight points. 
Let $X_{1}, \ldots, X_{n}$ be the monomials of degree $d$ in three variables $x_{0}, x_{1}, x_{2}$. For $D \in \mathcal{D}_{r}$, we can write

$$
f_{D}=\sum_{i=1}^{n} a_{i} \cdot X_{i}
$$

for suitable coefficients $a_{i}$, which we can calculate in the following way: If $p_{j}$ lies on $\pi(D)$, this gives a linear condition on the coefficients $a_{i}$ by substituting the coordinates of $p_{j}$ for $x_{0}, x_{1}, x_{2}$. If $p_{j}$ is a double point of $\pi(D)$, all partial derivatives of $f_{D}$ must vanish at this point, giving three more linear conditions. If $p_{j}$ is a triple point, we get six more linear conditions from the second derivatives. With $p_{1}, \ldots, p_{r}$ in general position, we check that these conditions determine $f_{D}$ uniquely up to a non-zero constant.

Relations corresponding to $(n)$-rulings. Suppose that an $(n)$-ruling $D$ can be written as $D_{j}+D_{j}^{\prime}$ for $k$ different pairs $D_{j}, D_{j}^{\prime} \in \mathcal{D}_{r}$ where $j \in$ $\{1, \ldots, k\}$. Then the products

$$
f_{D_{1}} \cdot f_{D_{1}^{\prime}}, \ldots, f_{D_{k}} \cdot f_{D_{k}^{\prime}}
$$

are $k$ homogeneous forms of the same degree $d$, and they span a vector space of dimension $n+1$ in the space of homogeneous polynomials of degree $d$. Consequently, there are $k-(n+1)$ independent relations between them, which we write as

$$
\sum_{j=1}^{k} a_{j, i} \cdot f_{D_{j}} \cdot f_{D_{j}^{\prime}}=0 \quad \text { for } i \in\{1, \ldots, k-(n+1)\} .
$$

for suitable constants $a_{j, i}$. They give an explicit description of the quadratic relations coming from $D$ :

Lemma 3.3. In this situation, the $(n)$-ruling $D$ gives the following $k-$ $(n+1)$ quadratic relations in $\operatorname{Cox}\left(S_{r}\right)$ :

$$
F_{D, i}:=\sum_{j=1}^{k} a_{j, i} \cdot \xi\left(D_{j}\right) \cdot \xi\left(D_{j}^{\prime}\right)=0 \quad \text { for } i \in\{1, \ldots, k-(n+1)\} .
$$

We will describe the $(n)$-rulings in more detail in the subsequent sections.

Let $J_{r}$ be the ideal in $R_{r}$ which is generated by the $(n)$-rulings (where $n=1$ for $r \leq 6, n \in\{1,2\}$ for $r=7$, and $n \in\{1,2,3\}$ for $r=8)$.

The proof of Theorem 3.2. For $r \in\{4,5,6\}$, this is BP04, Theorem 4.9]. For $r \in\{7,8\}$, we use a refinement of its proof.

Let $Z_{r}=\operatorname{Spec}\left(R_{r} / \operatorname{rad}\left(J_{r}\right)\right) \subset \operatorname{Spec}\left(R_{r}\right)$. We want to prove that $Z_{r}$ equals $\mathbb{A}\left(S_{r}\right) \subset \operatorname{Spec}\left(R_{r}\right)$, where $\mathbb{A}\left(S_{r}\right):=\operatorname{Spec}\left(\operatorname{Cox}\left(S_{r}\right)\right)$. Obviously, $0 \in$ $\operatorname{Spec}\left(R_{r}\right)$ is contained in both $Z_{r}$ and $\mathbb{A}\left(S_{r}\right)$. Its complement $\operatorname{Spec}\left(R_{r}\right) \backslash\{0\}$ is covered by the open sets

$$
U_{D}:=\{\xi(D) \neq 0\}, \quad \text { where } D \in \mathcal{D}_{r} .
$$

In the case $r=8$, we will show that it suffices to consider the sets $U_{D}$ for $D \in \mathcal{D}_{8} \backslash\left\{K_{1}, K_{2}\right\}$.

We want to show

$$
Z_{r} \cap U_{D} \cong Z_{r-1} \times\left(\mathbb{A}^{1} \backslash\{0\}\right) .
$$


Note that we can identify the $(-1)$-curves $\mathcal{D}_{r-1}$ of $S_{r-1}$ with the subset $\mathcal{D}_{r}^{\prime}$ of $\mathcal{D}_{r}$ containing the $(-1)$-curves which do not intersect $D$. We define

$$
\psi: \begin{array}{clc}
Z_{r} \cap U_{D} & \rightarrow & Z_{r-1} \times\left(\mathbb{A}^{1} \backslash\{0\}\right) \\
\left(\xi\left(D^{\prime}\right) \mid D^{\prime} \in \mathcal{D}_{r}\right) & \mapsto & \left(\left(\xi\left(D^{\prime}\right) \mid D^{\prime} \in \mathcal{D}_{r-1}\right), \xi(D)\right)
\end{array} .
$$

For $r \in\{7,8\}$, we will prove:

Lemma 3.4. Every $\xi\left(D^{\prime \prime}\right)$ for $D^{\prime \prime} \in \mathcal{D}_{r}$ intersecting $D$ is determined by

$$
\xi(D) \quad \text { and } \quad\left\{\xi\left(D^{\prime}\right) \mid D^{\prime} \in \mathcal{D}_{r} \quad \text { with } \quad\left(D^{\prime}, D\right)=0\right\},
$$

provided that $\xi(D) \neq 0$ and using the relations generating $J_{r}$.

By the proof of [BP04, Proposition 4.4],

$$
\mathbb{A}\left(S_{r}\right) \cap U_{D} \cong \mathbb{A}\left(S_{r-1}\right) \times\left(\mathbb{A}^{1} \backslash\{0\}\right) .
$$

By induction, $Z_{r-1}=\mathbb{A}\left(S_{r-1}\right)$. Therefore, $Z_{r} \cap U_{D}=\mathbb{A}\left(S_{r}\right) \cap U_{D}$ for every (-1)-curve $D$, which implies $Z_{r}=\mathbb{A}\left(S_{r}\right)$, completing the proof of Theorem 3.2 once Lemma 3.4 is proved.

\subsection{Degree 3}

We consider the case $r=6$, i.e., smooth cubic surfaces. By Lemma 1.14. the set $\mathcal{D}_{6}$ of $(-1)$-curves on $S_{6}$ consists of the following 27 divisors:

- exceptional divisors $E_{1}, \ldots, E_{6}$, preimages of $p_{1}, \ldots, p_{6} \in \mathbb{P}^{2}$,

- strict transforms $m_{i, j}=H-E_{i}-E_{j}$ of the 15 lines $m_{i, j}^{\prime}$ through the points $p_{i}, p_{j}(i \neq j \in\{1, \ldots, 6\})$, and

- strict transforms $Q_{k}=2 H-\left(E_{1}+\cdots+E_{6}\right)+E_{k}$ of the six conics $Q_{k}^{\prime}$ through all of the blown-up points except $p_{k}$.

With respect to the anticanonical embedding $S_{6} \hookrightarrow \mathbb{P}^{3}$, the $(-1)$-curves are the 27 lines (Remark 1.26).

Together with information from Section 3.2 , it is straightforward to derive:

Lemma 3.5. The extended Dynkin diagram of $(-1)$-curves has the following structure:

(1) It has 27 vertices corresponding to the 27 lines $E_{i}, m_{i, j}, Q_{i}$. Each of them has self-intersection number -1 .

(2) Every line intersects exactly 10 other lines: $E_{i}$ intersects $m_{i, j}$ and $Q_{j}($ for $j \neq i) ; m_{i, j}$ intersects $E_{i}, E_{j}, Q_{i}, Q_{j}$ and $m_{k, l}$ (for $\{i, j\} \cap$ $\{k, l\}=\emptyset) ; Q_{i}$ intersects $m_{i, j}$ and $E_{j}($ for $j \neq i)$. Correspondingly, there are 135 edges in the Dynkin diagram.

(3) There are 45 triangles, i.e., triples of lines which intersect pairwise: 30 triples $E_{i}, m_{i, j}, Q_{j}$ and 15 triples of the form $m_{i_{1}, j_{1}}, m_{i_{2}, j_{2}}, m_{i_{3}, j_{3}}$ where $\left\{i_{1}, j_{1}, i_{2}, j_{2}, i_{3}, j_{3}\right\}=\{1, \ldots, 6\}$. This corresponds to 45 triangles in the Dynkin diagram, where each edge is contained in exactly one of the triangles, and each vertex belongs to exactly five triangles.

Lemma 3.6. The 27 rulings of $S_{6}$ are given by $-K_{S_{6}}-D$ for $D \in \mathcal{D}_{6}$. Two (-1)-curves $D^{\prime}, D^{\prime \prime}$ fulfill $D^{\prime}+D^{\prime \prime}=-K_{S_{6}}-D$ if and only if $D, D^{\prime}, D^{\prime \prime}$ form a triangle in the sense of Lemma 3.5(3). There are five such pairs for any given $D$. 
Proof. We can check directly that $D+D^{\prime}+D^{\prime \prime}=-K_{S_{6}}$ if $D, D^{\prime}, D^{\prime \prime}$ form a triangle. Therefore, $-K_{S_{6}}-D$ is a ruling. As any $D$ is contained in exactly five triangles, this ruling can be expressed in five corresponding ways as $D^{\prime}+D^{\prime \prime}$.

On the other hand, by Table 3.1, the total number of rulings is 27 , and each ruling can be expressed in exactly five ways as the sum of two (-1)curves.

Let $D$ be one of the 27 lines of $S_{6}$, and consider the projection $\psi_{D}$ : $S_{6} \rightarrow \mathbb{P}^{1}$ from $D$. Then

$$
\psi_{D}^{*}\left(\mathcal{O}_{\mathbb{P}^{2}}(1)\right)=-K_{S_{6}}-D= \begin{cases}H-E_{i}, & D=Q_{i}, \\ 2 H-\left(E_{1}+\cdots+E_{6}\right)+E_{i}+E_{j}, & D=m_{i, j}, \\ 3 H-\left(E_{1}+\cdots+E_{6}\right)-E_{i}, & D=E_{i} .\end{cases}
$$

These are exactly the rulings.

A generating set of $\operatorname{Cox}\left(S_{6}\right)$ is given by section $\eta_{i}, \mu_{i, j}, \lambda_{i}$ corresponding to the 27 lines $E_{i}, m_{i, j}, Q_{i}$, respectively. We order them in the following way:

$$
\eta_{1}, \ldots, \eta_{6}, \quad \mu_{1,2}, \ldots, \mu_{1,6}, \mu_{2,3}, \ldots, \mu_{2,6}, \mu_{3,4}, \ldots, \mu_{5,6}, \quad \lambda_{1}, \ldots, \lambda_{6} .
$$

Let

$$
R_{6}:=\mathbb{K}\left[\eta_{i}, \mu_{i, j}, \lambda_{i}\right] .
$$

The quadratic monomials in $H^{0}\left(S_{6},-K_{S_{6}}-D\right)$ corresponding to the five ways to express $-K_{S_{6}}-D$ as the sum of the $(-1)$-curves are

- $\mu_{i, j} \eta_{j}$ if $D=Q_{i}$

- $\eta_{i} \lambda_{j}, \eta_{j} \lambda_{i}, \mu_{k_{1}, k_{2}} \mu_{k_{3}, k_{4}}$ if $D=\mu_{i, j}\left(\left\{i, j, k_{1}, \ldots, k_{4}\right\}=\{1, \ldots, 6\}\right)$

- $\mu_{i, j} \lambda_{j}$ if $D=E_{i}$

In order to calculate the 81 relations in $J_{6}$ explicitly as described in Lemma 3.3, we use the coordinates of (3.1) for $p_{1}, \ldots, p_{4}$, and

$$
p_{5}=(1: a: b), \quad p_{6}=(1: c: d) .
$$

We write

$$
E:=(b-1)(c-1)-(a-1)(d-1) \quad \text { and } \quad F:=b c-a d
$$

for simplicity. The three relations corresponding to a line $D$ are denoted by

$$
\begin{gathered}
F_{-K_{S_{6}}-D, 1}, F_{-K_{S_{6}}-D, 2}, F_{-K_{S_{6}}-D, 3} . \\
F_{-K_{S_{6}}-Q_{1}, 1}=-\eta_{2} \mu_{1,2}-\eta_{3} \mu_{1,3}+\eta_{4} \mu_{1,4} \\
F_{-K_{S_{6}}-Q_{1}, 2}=-a \eta_{2} \mu_{1,2}-b \eta_{3} \mu_{1,3}+\eta_{5} \mu_{1,5} \\
F_{-K_{S_{6}}-Q_{1}, 2}=-c \eta_{2} \mu_{1,2}-d \eta_{3} \mu_{1,3}+\eta_{6} \mu_{1,6} \\
F_{-K_{S_{6}}-Q_{2}, 1}=\eta_{1} \mu_{1,2}-\eta_{3} \mu_{2,3}+\eta_{4} \mu_{2,4} \\
F_{-K_{S_{6}}-Q_{2}, 2}=\eta_{1} \mu_{1,2}-b \eta_{3} \mu_{2,3}+\eta_{5} \mu_{2,5} \\
F_{-K_{S_{6}}}-Q_{2}, 3 \\
=\eta_{1} \mu_{1,2}-d \eta_{3} \mu_{2,3}+\eta_{6} \mu_{2,6} \\
F_{-K_{S_{6}}-Q_{3}, 1}=\eta_{1} \mu_{1,3}+\eta_{2} \mu_{2,3}+\eta_{4} \mu_{3,4} \\
F_{-K_{S_{6}}-Q_{3}, 2}=\eta_{1} \mu_{1,3}+a \eta_{2} \mu_{2,3}+\eta_{5} \mu_{3,5} \\
F_{-K_{S_{6}}-Q_{3}, 3}=\eta_{1} \mu_{1,3}+c \eta_{2} \mu_{2,3}+\eta_{6} \mu_{3,6}
\end{gathered}
$$




$$
\begin{aligned}
& F_{-K_{S_{6}}-Q_{4}, 1}=\eta_{1} \mu_{1,4}+\eta_{2} \mu_{2,4}+\eta_{3} \mu_{3,4} \\
& F_{-K_{S_{6}}-Q_{4}, 2}=(1-b) \eta_{1} \mu_{1,4}+(a-b) \eta_{2} \mu_{2,4}+\eta_{5} \mu_{4,5} \\
& F_{-K_{S_{6}}-Q_{4}, 3}=(1-d) \eta_{1} \mu_{1,4}+(c-d) \eta_{2} \mu_{2,4}+\eta_{6} \mu_{4,6} \\
& F_{-K_{S_{6}}-Q_{5}, 1}=1 / b \eta_{1} \mu_{1,5}+a / b \eta_{2} \mu_{2,5}+\eta_{3} \mu_{3,5} \\
& F_{-K_{S_{6}}-Q_{5}, 2}=(1-b) / b \eta_{1} \mu_{1,5}+(a-b) / b \eta_{2} \mu_{2,5}+\eta_{4} \mu_{4,5} \\
& F_{-K_{S_{6}}-Q_{5}, 3}=(b-d) / b \eta_{1} \mu_{1,5}+F / b \eta_{2} \mu_{2,5}+\eta_{6} \mu_{5,6} \\
& F_{-K_{S_{6}}-Q_{6}, 1}=1 / d \eta_{1} \mu_{1,6}+c / d \eta_{2} \mu_{2,6}+\eta_{3} \mu_{3,6} \\
& F_{-K_{S_{6}}-Q_{6}, 2}=(1-d) / d \eta_{1} \mu_{1,6}+(c-d) / d \eta_{2} \mu_{2,6}+\eta_{4} \mu_{4,6} \\
& F_{-K_{S_{6}}-Q_{6}, 3}=(b-d) / d \eta_{1} \mu_{1,6}+F / d \eta_{2} \mu_{2,6}+\eta_{5} \mu_{5,6} \\
& F_{-K_{S_{6}}-m_{1,2}, 1}=\mu_{4,5} \mu_{3,6}-\mu_{3,5} \mu_{4,6}+\mu_{3,4} \mu_{5,6} \\
& F_{-K_{S_{6}}-m_{1,2}, 2}=(b-d) \mu_{3,5} \mu_{4,6}+(d-1) \mu_{3,4} \mu_{5,6}+\eta_{2} \lambda_{1} \\
& F_{-K_{S_{6}}-m_{1,2}, 3}=F \mu_{3,5} \mu_{4,6}+a(d-c) \mu_{3,4} \mu_{5,6}+\eta_{1} \lambda_{2} \\
& F_{-K_{S_{6}}-m_{1,3}, 1}=\mu_{4,5} \mu_{2,6}-\mu_{2,5} \mu_{4,6}+\mu_{2,4} \mu_{5,6} \\
& F_{-K_{S_{6}}-m_{1,3}, 2}=(c-a) \mu_{2,5} \mu_{4,6}+(1-c) \mu_{2,4} \mu_{5,6}+\eta_{3} \lambda_{1} \\
& F_{-K_{S_{6}}-m_{1,3}, 3}=-F \mu_{2,5} \mu_{4,6}+b(c-d) \mu_{2,4} \mu_{5,6}+\eta_{1} \lambda_{3} \\
& F_{-K_{S_{6}}-m_{2,3}, 1}=\mu_{4,5} \mu_{1,6}-\mu_{1,5} \mu_{4,6}+\mu_{1,4} \mu_{5,6} \\
& F_{-K_{S_{6}}-m_{2,3}, 2}=(a-c) \mu_{1,5} \mu_{4,6}+a(c-1) \mu_{1,4} \mu_{5,6}+\eta_{3} \lambda_{2} \\
& F_{-K_{S_{6}}-m_{2,3}, 3}=(b-d) \mu_{1,5} \mu_{4,6}+b(d-1) \mu_{1,4} \mu_{5,6}+\eta_{2} \lambda_{3} \\
& F_{-K_{S_{6}}-m_{1,4}, 1}=\mu_{3,5} \mu_{2,6}-\mu_{2,5} \mu_{3,6}+\mu_{2,3} \mu_{5,6} \\
& F_{-K_{S_{6}}-m_{1,4}, 2}=-E \mu_{2,5} \mu_{3,6}+(b-1)(c-1) \mu_{2,3} \mu_{5,6}+\eta_{4} \lambda_{1} \\
& F_{-K_{S_{6}}-m_{1,4}, 3}=-F \mu_{2,5} \mu_{3,6}+b c \mu_{2,3} \mu_{5,6}+\eta_{1} \lambda_{4} \\
& F_{-K_{S_{6}}-m_{2,4}, 1}=\mu_{3,5} \mu_{1,6}-\mu_{1,5} \mu_{3,6}+\mu_{1,3} \mu_{5,6} \\
& F_{-K_{S_{6}}-m_{2,4}, 2}=E \mu_{1,5} \mu_{3,6}+(a-b)(c-1) \mu_{1,3} \mu_{5,6}+\eta_{4} \lambda_{2} \\
& F_{-K_{S_{6}}-m_{2,4}, 3}=(b-d) \mu_{1,5} \mu_{3,6}-b \mu_{1,3} \mu_{5,6}+\eta_{2} \lambda_{4} \\
& F_{-K_{S_{6}}-m_{3,4}, 1}=\mu_{2,5} \mu_{1,6}-\mu_{1,5} \mu_{2,6}+\mu_{1,2} \mu_{5,6} \\
& F_{-K_{S_{6}}-m_{3,4}, 2}=-E \mu_{1,5} \mu_{2,6}+(a-b)(1-d) \mu_{1,2} \mu_{5,6}+\eta_{4} \lambda_{3} \\
& F_{-K_{S_{6}}-m_{3,4}, 3}=(c-a) \mu_{1,5} \mu_{2,6}+a \mu_{1,2} \mu_{5,6}+\eta_{3} \lambda_{4}
\end{aligned}
$$




$$
\begin{aligned}
& F_{-K_{S_{6}}-m_{1,5}, 1}=\mu_{3,4} \mu_{2,6}-\mu_{2,4} \mu_{3,6}+\mu_{2,3} \mu_{4,6} \\
& F_{-K_{S_{6}}-m_{1,5}, 2}=-E \mu_{2,4} \mu_{3,6}+(a-c)(1-b) \mu_{2,3} \mu_{4,6}+\eta_{5} \lambda_{1} \\
& F_{-K_{S_{6}}-m_{1,5}, 3}=(d-c) \mu_{2,4} \mu_{3,6}+c \mu_{2,3} \mu_{4,6}+\eta_{1} \lambda_{5} \\
& F_{-K_{S_{6}}-m_{2,5}, 1}=\mu_{3,4} \mu_{1,6}-\mu_{1,4} \mu_{3,6}+\mu_{1,3} \mu_{4,6} \\
& F_{-K_{S_{6}}-m_{2,5}, 2}=a E \mu_{1,4} \mu_{3,6}+(a-b)(c-a) \mu_{1,3} \mu_{4,6}+\eta_{5} \lambda_{2} \\
& F_{-K_{S_{6}}-m_{2,5}, 3}=(1-d) \mu_{1,4} \mu_{3,6}-\mu_{1,3} \mu_{4,6}+\eta_{2} \lambda_{5} \\
& F_{-K_{S_{6}}-m_{3,5}, 1}=\mu_{2,4} \mu_{1,6}-\mu_{1,4} \mu_{2,6}+\mu_{1,2} \mu_{4,6} \\
& F_{-K_{S_{6}}-m_{3,5}, 2}=-b E \mu_{1,4} \mu_{2,6}+(a-b)(b-d) \mu_{1,2} \mu_{4,6}+\eta_{5} \lambda_{3} \\
& F_{-K_{S_{6}}-m_{3,5}, 3}=(c-1) \mu_{1,4} \mu_{2,6}+\mu_{1,2} \mu_{4,6}+\eta_{3} \lambda_{5} \\
& F_{-K_{S_{6}}-m_{4,5}, 1}=\mu_{2,3} \mu_{1,6}-\mu_{1,3} \mu_{2,6}+\mu_{1,2} \mu_{3,6} \\
& F_{-K_{S_{6}}-m_{4,5}, 2}=b(c-a) \mu_{1,3} \mu_{2,6}+a(b-d) \mu_{1,2} \mu_{3,6}+\eta_{5} \lambda_{4} \\
& F_{-K_{S_{6}}-m_{4,5}, 3}=(c-1) \mu_{1,3} \mu_{2,6}+(1-d) \mu_{1,2} \mu_{3,6}+\eta_{4} \lambda_{5} \\
& F_{-K_{S_{6}}-m_{1,6}, 1}=\mu_{3,4} \mu_{2,5}-\mu_{2,4} \mu_{3,5}+\mu_{2,3} \mu_{4,5} \\
& F_{-K_{S_{6}}-m_{1,6}, 2}=-E \mu_{2,4} \mu_{3,5}+(a-c)(1-d) \mu_{2,3} \mu_{4,5}+\eta_{6} \lambda_{1} \\
& F_{-K_{S_{6}}-m_{1,6}, 3}=(b-a) \mu_{2,4} \mu_{3,5}+a \mu_{2,3} \mu_{4,5}+\eta_{1} \lambda_{6} \\
& F_{-K_{S_{6}}-m_{2,6}, 1}=\mu_{3,4} \mu_{1,5}-\mu_{1,4} \mu_{3,5}+\mu_{1,3} \mu_{4,5} \\
& F_{-K_{S_{6}}-m_{2,6}, 2}=c E \mu_{1,4} \mu_{3,5}+(a-c)(d-c) \mu_{1,3} \mu_{4,5}+\eta_{6} \lambda_{2} \\
& F_{-K_{S_{6}}-m_{2,6}, 3}=(1-b) \mu_{1,4} \mu_{3,5}-\mu_{1,3} \mu_{4,5}+\eta_{2} \lambda_{6} \\
& F_{-K_{S_{6}}-m_{3,6}, 1}=\mu_{2,4} \mu_{1,5}-\mu_{1,4} \mu_{2,5}+\mu_{1,2} \mu_{4,5} \\
& F_{-K_{S_{6}}-m_{3,6}, 2}=-d E \mu_{1,4} \mu_{2,5}+(d-b)(d-c) \mu_{1,2} \mu_{4,5}+\eta_{6} \lambda_{3} \\
& F_{-K_{S_{6}}-m_{3,6}, 3}=(a-1) \mu_{1,4} \mu_{2,5}+\mu_{1,2} \mu_{4,5}+\eta_{3} \lambda_{6} \\
& F_{-K_{S_{6}}-m_{4,6}, 1}=\mu_{2,3} \mu_{1,5}-\mu_{1,3} \mu_{2,5}+\mu_{1,2} \mu_{3,5} \\
& F_{-K_{S_{6}}-m_{4,6}, 2}=d(c-a) \mu_{1,3} \mu_{2,5}+c(b-d) \mu_{1,2} \mu_{3,5}+\eta_{6} \lambda_{4} \\
& F_{-K_{S_{6}}-m_{4,6}, 3}=(a-1) \mu_{1,3} \mu_{2,5}+(1-b) \mu_{1,2} \mu_{3,5}+\eta_{4} \lambda_{6} \\
& F_{-K_{S_{6}}-m_{5,6}, 1}=\mu_{2,3} \mu_{1,4}-\mu_{1,3} \mu_{2,4}+\mu_{1,2} \mu_{3,4} \\
& F_{-K_{S_{6}}-m_{5,6}, 2}=d(c-1) \mu_{1,3} \mu_{2,4}+c(1-d) \mu_{1,2} \mu_{3,4}+\eta_{6} \lambda_{5} \\
& F_{-K_{S_{6}}-m_{5,6}, 3}=b(a-1) \mu_{1,3} \mu_{2,4}+a(1-b) \mu_{1,2} \mu_{3,4}+\eta_{5} \lambda_{6}
\end{aligned}
$$




$$
\begin{aligned}
& F_{-K_{S_{6}}-E_{1}, 1}=(d-b) / E \mu_{1,2} \lambda_{2}+(c-a) / E \mu_{1,3} \lambda_{3}+\mu_{1,4} \lambda_{4} \\
& F_{-K_{S_{6}}-E_{1}, 2}=(d-1) / E \mu_{1,2} \lambda_{2}+(c-1) / E \mu_{1,3} \lambda_{3}+\mu_{1,5} \lambda_{5} \\
& F_{-K_{S_{6}}-E_{1}, 3}=(b-1) / E \mu_{1,2} \lambda_{2}+(a-1) / E \mu_{1,3} \lambda_{3}+\mu_{1,6} \lambda_{6} \\
& F_{-K_{S_{6}}-E_{2}, 1}=F / E \mu_{1,2} \lambda_{1}+(c-a) / E \mu_{2,3} \lambda_{3}+\mu_{2,4} \lambda_{4} \\
& F_{-K_{S_{6}}-E_{2}, 2}=(c-d) / E \mu_{1,2} \lambda_{1}+(c-1) / E \mu_{2,3} \lambda_{3}+\mu_{2,5} \lambda_{5} \\
& F_{-K_{S_{6}}-E_{2}, 3}=(a-b) / E \mu_{1,2} \lambda_{1}+(a-1) / E \mu_{2,3} \lambda_{3}+\mu_{2,6} \lambda_{6} \\
& F_{-K_{S_{6}}-E_{3}, 1}=F / E \mu_{1,3} \lambda_{1}+(b-d) / E \mu_{2,3} \lambda_{2}+\mu_{3,4} \lambda_{4} \\
& F_{-K_{S_{6}}-E_{3}, 2}=(c-d) / E \mu_{1,3} \lambda_{1}+(1-d) / E \mu_{2,3} \lambda_{2}+\mu_{3,5} \lambda_{5} \\
& F_{-K_{S_{6}}-E_{3}, 3}=(a-b) / E \mu_{1,3} \lambda_{1}+(1-b) / E \mu_{2,3} \lambda_{2}+\mu_{3,6} \lambda_{6} \\
& F_{-K_{S_{6}}-E_{4}, 1}=F /(a-c) \mu_{1,4} \lambda_{1}+(b-d) /(a-c) \mu_{2,4} \lambda_{2}+\mu_{3,4} \lambda_{3} \\
& F_{-K_{S_{6}}-E_{4}, 2}=c /(a-c) \mu_{1,4} \lambda_{1}+1 /(a-c) \mu_{2,4} \lambda_{2}+\mu_{4,5} \lambda_{5} \\
& F_{-K_{S_{6}}-E_{4}, 3}=a /(a-c) \mu_{1,4} \lambda_{1}+1 /(a-c) \mu_{2,4} \lambda_{2}+\mu_{4,6} \lambda_{6} \\
& F_{-K_{S_{6}}-E_{5}, 1}=(d-c) /(c-1) \mu_{1,5} \lambda_{1}+(d-1) /(c-1) \mu_{2,5} \lambda_{2}+\mu_{3,5} \lambda_{3} \\
& F_{-K_{S_{6}}-E_{5}, 2}=-c /(c-1) \mu_{1,5} \lambda_{1}-1 /(c-1) \mu_{2,5} \lambda_{2}+\mu_{4,5} \lambda_{4} \\
& F_{-K_{S_{6}}-E_{5}, 3}=-1 /(c-1) \mu_{1,5} \lambda_{1}-1 /(c-1) \mu_{2,5} \lambda_{2}+\mu_{5,6} \lambda_{6} \\
& F_{-K_{S_{6}}-E_{6}, 1}=(b-a) /(a-1) \mu_{1,6} \lambda_{1}+(b-1) /(a-1) \mu_{2,6} \lambda_{2}+\mu_{3,6} \lambda_{3} \\
& F_{-K_{S_{6}}-E_{6}, 2}=-a /(a-1) \mu_{1,6} \lambda_{1}-1 /(a-1) \mu_{2,6} \lambda_{2}+\mu_{4,6} \lambda_{4} \\
& F_{-K_{S_{6}}-E_{6}, 3}=-1 /(a-1) \mu_{1,6} \lambda_{1}-1 /(a-1) \mu_{2,6} \lambda_{2}+\mu_{5,6} \lambda_{5}
\end{aligned}
$$

\subsection{Degree 2}

Let $S_{7}$ be a smooth Del Pezzo surface of degree $d=2$, i.e., the blow-up of $\mathbb{P}^{2}$ in $r=7$ points. The set $\mathcal{D}_{7}$ contains $56(-1)$-curves which are the strict transforms of the following curves in $\mathbb{P}^{2}$ :

- blow-ups $E_{1}, \ldots, E_{7}$ of $p_{1}, \ldots, p_{7}$;

- 21 lines $m_{i, j}^{\prime}$ through $p_{i}, p_{j}$, where

$$
m_{i, j}=H-E_{i}-E_{j}
$$

- 21 conics $Q_{i, j}^{\prime}$ through five of the seven points, missing $p_{i}, p_{j}$, where

$$
Q_{i, j}=2 H-\left(E_{1}+\cdots+E_{7}\right)+E_{i}+E_{j} ;
$$

- 7 singular cubics $C_{i}^{\prime}$ through all seven points, where $p_{i}$ is a double point, and

$$
C_{i}=3 H-\left(E_{1}+\cdots+E_{7}\right)-E_{i} .
$$

The Cox ring $\operatorname{Cox}\left(S_{7}\right)$ is generated by the sections $\eta_{i}, \mu_{i, j}, \nu_{i, j}, \lambda_{i}$ corresponding to the $56(-1)$-curves $E_{i}, m_{i, j}, Q_{i, j}, C_{i}$, respectively. They are 
ordered as

$\eta_{1}, \ldots, \eta_{7}, \mu_{1,2}, \ldots, \mu_{1,7}, \mu_{2,3}, \ldots, \mu_{6,7}, \nu_{1,2}, \ldots, \nu_{1,7}, \nu_{2,3}, \ldots, \nu_{6,7}, \lambda_{1}, \ldots, \lambda_{7}$.

Let

$$
R_{7}:=\mathbb{K}\left[\eta_{i}, \mu_{i, j}, \nu_{i, j}, \lambda_{i}\right]
$$

be the polynomial ring in 56 generators.

Consider the ideal $I_{7} \subset R_{7}$ generated by the quadratic relations corresponding to rulings. In view of Lemma 3.3 , we need to know the six different ways to write each of the 126 rulings as a sum of two (-1)-curves in order to describe $I_{7}$ explicitly. Here, we do not write the resulting 504 relations down because of the length of this list.

LEMMA 3.7. Each of the 126 rulings can be written in six ways as a sum of two (-1)-curves:

(1) For the seven rulings $H-E_{i}$ :

$$
\left\{E_{j}+m_{i, j} \mid j \neq i\right\} \text {. }
$$

(2) For the 35 rulings $2 H-\left(E_{1}+\cdots+E_{7}\right)+E_{i}+E_{j}+E_{k}$ :

$$
\left\{E_{i}+Q_{j, k}, E_{j}+Q_{i, k}, E_{k}+Q_{i, j}, m_{l_{1}, l_{2}}+m_{l_{3}, l_{4}} \mid\left\{i, j, k, l_{1}, l_{2}, l_{3}, l_{4}\right\}=\{1, \ldots, 7\}\right\}
$$

(3) For the 42 rulings $3 H-\left(E_{1}+\cdots+E_{7}\right)+E_{i}-E_{j}$ :

$$
\left\{E_{i}+C_{j}, Q_{i, k}+m_{j, k} \mid k \neq i, j\right\} .
$$

(4) For the 35 rulings $4 H-\left(E_{1}+\cdots+E_{7}\right)-E_{i}-E_{j}-E_{k}$ :

$$
\left\{C_{i}+m_{j, k}, C_{j}+m_{i, k}, C_{k}+m_{i, j}, Q_{l_{1}, l_{2}}+Q_{l_{3}, l_{4}} \mid\left\{i, j, k, l_{1}, l_{2}, l_{3}, l_{4}\right\}=\{1, \ldots, 7\}\right\}
$$

(5) For the seven rulings $5 H-2\left(E_{1}+\cdots+E_{7}\right)+E_{i}$ :

$$
\left\{C_{j}+Q_{i, j} \mid j \neq i\right\} \text {. }
$$

However, we have more quadratic relations in $\operatorname{Cox}\left(S_{7}\right)$ : Note that the point $q$, with $\eta_{1}=\lambda_{1}=1$ and other coordinates zero, satisfies the 504 relations. Indeed, $\left(E_{1}, C_{1}\right)=2$, but all quadratic monomials which occur in the relations correspond to pairs of divisors whose intersection number is 1 . Hence, all these monomials and all the relations vanish in $q$. On the other hand, we check that the $504 \times 56$ Jacobian matrix has rank 54 in this point, which means that $q$ is contained in a component of the variety defined by $I_{7}$ which has dimension 2 . As $\mathbb{A}\left(S_{7}\right)$ is irreducible of dimension 10 , we must find other relations to exclude such components.

As $E_{1}+C_{1}=-K_{S_{7}}$, we look for more relations in degree $-K_{S_{7}}$ of $\operatorname{Cox}\left(S_{7}\right)$ : We check that in this degree, we have exactly 28 monomials:

$$
\left\{\eta_{i} \lambda_{i} \mid 1 \leq i \leq 7\right\} \cup\left\{\mu_{j, k} \nu_{j, k} \mid 1 \leq j<k \leq 7\right\},
$$

corresponding to $-K_{S_{7}}=E_{i}+C_{i}=m_{j, k}+Q_{j, k}$. As $\operatorname{dim} H^{0}\left(S_{7},-K_{S_{7}}\right)=3$, and as none of the relations coming from rulings induces a relation in this degree, we obtain 25 independent relations. Note that $-K_{S_{7}}$ is the unique (2)-ruling of $S_{7}$.

We can calculate the relations explicitly as they correspond to the relations between the polynomials $f_{E_{i}} \cdot f_{C_{i}}$ and $f_{m_{i, j}} \cdot f_{Q_{i, j}}$, which are homogeneous of degree 3 , as described in Lemma 3.3 .

Let $J_{7}$ be the ideal generated by these 529 relations. 
Proof of Lemma 3.4. In order to show that $\operatorname{Cox}\left(S_{7}\right)$ is described by $\operatorname{rad}\left(J_{7}\right)$, we must prove Lemma 3.4 in the case $r=7$.

For any $D \in \mathcal{D}_{7}$, consider a coordinate $\xi\left(D^{\prime}\right)$ where $\left(D, D^{\prime}\right)=1$. This is determined by the ruling $D+D^{\prime}$. Indeed, this ruling induces a relation of the form

$$
\xi(D) \xi\left(D^{\prime}\right)=\sum a_{i} \xi\left(D_{i}\right) \xi\left(D_{i}^{\prime}\right),
$$

where $D_{i}+D_{i}^{\prime}=D+D^{\prime}$. Therefore,

$$
\left(D, D_{i}+D_{i}^{\prime}\right)=\left(D, D+D^{\prime}\right)=(D, D)+\left(D, D^{\prime}\right)=-1+1=0,
$$

which implies $\left(D, D_{i}\right)=\left(D, D_{i}^{\prime}\right)=0$ since the only $(-1)$-curve intersecting $D$ negatively is $D$ itself. Since $\xi(D) \neq 0$, the only unknown variable $\xi\left(D^{\prime}\right)$ is determined by this relation.

Furthermore, there is exactly one coordinate $\xi\left(D^{\prime \prime}\right)$ where $\left(D, D^{\prime \prime}\right)=2$. The unique (2)-ruling $D+D^{\prime \prime}=-K_{S_{7}}$ induces a relation of the form

$$
\xi(D) \xi\left(D^{\prime \prime}\right)=\sum a_{i} \xi\left(D_{i}\right) \xi\left(D_{i}^{\prime}\right)
$$

where $\xi\left(D^{\prime \prime}\right)$ is the only unknown variable.

\subsection{Degree 1}

In this section, we consider blow-ups of $\mathbb{P}^{2}$ in $r=8$ points in general position, i.e., smooth Del Pezzo surfaces $S_{8}$ of degree 1.

The set $\mathcal{D}_{8}$ contains the strict transforms of the following 242 curves:

- Blow-ups $E_{1}, \ldots, E_{8}$ of $p_{1}, \ldots, p_{8}$;

- 28 lines $m_{i, j}^{\prime}$ through $p_{i}, p_{j}$ :

$$
m_{i, j}=H-E_{i}-E_{j}
$$

- 56 conics $Q_{i, j, k}^{\prime}$ through 5 points, missing $p_{i}, p_{j}, p_{k}$ :

$$
Q_{i, j, k}=2 H-\left(E_{1}+\cdots+E_{8}\right)+E_{i}+E_{j}+E_{k} ;
$$

- 56 cubics $C_{i, j}^{\prime}$ through 7 points missing $p_{j}$, where $p_{i}$ is a double point:

$$
C_{i, j}=3 H-\left(E_{1}+\cdots+E_{8}\right)-E_{i}+E_{j}
$$

- 56 quartics $V_{i, j, k}^{\prime}$ through all points, where $p_{i}, p_{j}, p_{k}$ are double points:

$$
V_{i, j, k}=4 H-\left(E_{1}+\cdots+E_{8}\right)-\left(E_{i}+E_{j}+E_{k}\right) ;
$$

- 28 quintics $F_{i, j}^{\prime}$ through all points, where $p_{i}, p_{j}$ are simple points and the other six are double points:

$$
F_{i, j}=5 H-2\left(E_{1}+\cdots+E_{8}\right)+E_{i}+E_{j} ;
$$

- 8 sextics $T_{i}^{\prime}$, where $p_{i}$ a triple point and the other seven points are double points:

$$
T_{i}=6 H-2\left(E_{1}+\cdots+E_{8}\right)-E_{i} ;
$$

- two independent cubics $K_{1}^{\prime}, K_{2}^{\prime}$ through the eight points:

$$
\left[K_{1}\right]=\left[K_{2}\right]=-K_{S_{8}}=3-\left(E_{1}+\cdots+E_{8}\right) .
$$


The Cox ring of $S_{8}$ is generated by the 242 sections

$$
\eta_{i}, \mu_{i, j}, \nu_{i, j, k}, \lambda_{i, j}, \phi_{i, j, k}, \psi_{i, j}, \sigma_{i}, \kappa_{i}
$$

of $E_{i}, m_{i, j}, Q_{i, j, k}, C_{i, j}, V_{i, j, k}, F_{i, j}, T_{i}, K_{i}$, respectively.

LEMMA 3.8. Each of the 2160 rulings can be expressed in the following seven ways as a sum of two (-1)-curves:

- 8 rulings of the form $H-E_{i}$ :

$$
\left\{E_{j}+m_{i, j} \mid j \neq i\right\} \text {. }
$$

- $\left(\begin{array}{l}8 \\ 4\end{array}\right)=70$ rulings of the form $2 H-\left(E_{i}+E_{j}+E_{k}+E_{l}\right)$ :

$$
\left\{\begin{array}{c}
m_{i, j}+m_{k, l}, m_{i, k}+m_{j, l}, \\
m_{i, l}+m_{j, k}, E_{a}+Q_{b, c, d}
\end{array} \mid\{a, b, c, d, i, j, k, l\}=\{1, \ldots, 8\}\right\} \text {. }
$$

- $8 \cdot\left(\begin{array}{l}7 \\ 2\end{array}\right)=168$ rulings of the form $3 H-\left(E_{1}+\cdots+E_{8}\right)-E_{i}+E_{j}+E_{k}$ :

$$
\left\{E_{j}+C_{i, k}, E_{k}+C_{i, j}, m_{i, l}+Q_{j, k, l} \mid l \notin\{i, j, k\}\right\} .
$$

- $8 \cdot\left(\begin{array}{l}7 \\ 3\end{array}\right)=280$ rulings $4 H-\left(E_{1}+\cdots+E_{8}\right)+E_{i}-\left(E_{j}+E_{k}+E_{l}\right)$ :

$$
\left\{\begin{array}{l}
E_{i}+V_{j, k, l}, Q_{i, a, b}+Q_{i, c, d}, \\
C_{j, i}+m_{k, l}, C_{k, i}+m_{j, l}, C_{l, i}+m_{j, k}
\end{array} \mid\{a, b, c, d, i, j, k, l\}=\{1, \ldots, 8\}\right\},
$$

and 8 rulings of the form $4 H-\left(E_{1}+\cdots+E_{8}\right)-2 E_{i}$ :

$$
\left\{m_{i, j}+C_{i, j} \mid j \neq i\right\} \text {. }
$$

- $8 \cdot 7=56$ rulings of the form $5 H-2\left(E_{1}+\cdots+E_{8}\right)+2 E_{i}+E_{j}$ :

$$
\left\{E_{i}+F_{i, j}, C_{k, i}+Q_{i, j, k} \mid k \notin\{i, j\}\right\},
$$

and $8 \cdot\left(\begin{array}{l}7 \\ 3\end{array}\right)=280$ rulings $5 H-\left(E_{1}+\cdots+E_{8}\right)-2 E_{i}-\left(E_{j}+E_{k}+E_{l}\right)$ :

$\left\{\begin{array}{l}m_{i, j}+V_{i, k, l}, m_{i, k}+V_{i, j, l} \\ m_{i, l}+V_{i, j, k}, C_{i, a}+Q_{b, c, d}\end{array} \mid\{a, b, c, d, i, j, k, l\}=\{1, \ldots, 8\}\right\}$.

- $\left(\begin{array}{l}8 \\ 2\end{array}\right) \cdot\left(\begin{array}{l}6 \\ 2\end{array}\right)=420$ rulings $6 H-2\left(E_{1}+\cdots+E_{8}\right)-\left(E_{i}+E_{j}\right)+E_{k}+E_{l}$ :

$\left\{m_{i, j}+F_{k, l}, V_{i, j, m}+Q_{k, l, m}, C_{i, k}+C_{j, l}, C_{i, l}+C_{j, k} \mid m \notin\{i, j, k, l\}\right\}$.

- $8 \cdot 7=56$ rulings of the form $7 H-2\left(E_{1}+\cdots+E_{8}\right)-2 E_{i}-E_{j}$ :

$$
\left\{m_{i, j}+T_{i}, C_{i, k}+V_{i, j, k} \mid k \notin\{i, j\}\right\},
$$

and $8 \cdot\left(\begin{array}{l}7 \\ 3\end{array}\right)=280$ rulings $7 H-3\left(E_{1}+\cdots+E_{8}\right)+2 E_{i}+E_{j}+E_{k}+E_{l}$ :

$$
\left\{\begin{array}{c}
F_{i, j}+Q_{i, k, l}, F_{i, k}+Q_{i, j, l} \\
F_{i, l}+Q_{i, j, k}, C_{a, i}+V_{b, c, d}
\end{array} \mid\{a, b, c, d, i, j, k, l\}=\{1, \ldots, 8\}\right\} .
$$

- $8 \cdot\left(\begin{array}{l}7 \\ 3\end{array}\right)=280$ rulings $8 H-3\left(E_{1}+\cdots+E_{8}\right)-E_{i}+E_{j}+E_{k}+E_{l}$ :

$$
\left\{\begin{array}{l}
C_{i, j}+F_{k, l}, C_{i, k}+F_{j, l}, C_{i, l}+F_{j, k}, \\
T_{i}+Q_{j, k, l}, V_{i, a, b}+V_{i, c, d}
\end{array} \mid\{a, b, c, d, i, j, k, l\}=\{1, \ldots, 8\}\right\},
$$

and 8 rulings of the form $8 H-3\left(E_{1}+\cdots+E_{8}\right)+2 E_{i}$ :

$$
\left\{F_{i, j}+C_{j, i} \mid j \neq i\right\} \text {. }
$$


- $8 \cdot\left(\begin{array}{l}7 \\ 2\end{array}\right)=168$ rulings of the form $9 H-3\left(E_{1}+\cdots+E_{8}\right)+E_{i}-\left(E_{j}+E_{k}\right)$ :

$$
\left\{S_{j}+C_{k, i}, S_{k}+C_{j, i}, F_{i, l}+V_{j, k, l} \mid l \notin\{i, j, k\}\right\} .
$$

- $\left(\begin{array}{l}8 \\ 4\end{array}\right)=70$ rulings of the form $10 H-4\left(E_{1}+\cdots+E_{8}\right)+E_{i}+E_{j}+E_{k}+E_{l}$ : $\left\{F_{i, j}+F_{k, l}, F_{i, k}+F_{j, l}, F_{i, l}+F_{j, k}, S_{a}+V_{b, c, d} \mid\{a, b, c, d, i, j, k, l\}=\{1, \ldots, 8\}\right\}$.

- 8 rulings of the form $11 H-4\left(E_{1}+\cdots+E_{8}\right)+E_{i}$ :

$$
\left\{S_{j}+F_{i, j} \mid j \neq i\right\} \text {. }
$$

There is no way to write a ruling as the sum of $-K_{S_{8}}$ and $(-1)$-curves.

Proof. Because of the Weyl group symmetry, we need to prove the last statement only in one case, say $H-E_{1}$. In this case, it is obvious.

By Table 3.1. there can be no other rulings, and each ruling can be expressed in no further ways as the sum of two (-1)-curves.

With this information, Lemma 3.3 allows us to determine the 10800 relations coming from rulings explicitly.

We can find more quadratic relations in the degrees corresponding to (2)-rulings: Because of the Weyl group symmetry, it is enough to consider the (2)-ruling $D:=E_{2}+C_{2,1}$. This can also be written as $E_{j}+C_{j, 1}$ for any $j \neq 1$ and as $m_{i, j}+Q_{1, i, j}$ for any $i, j \neq 1$, giving 28 section in $H^{0}\left(S_{8}, D\right)$. As $D=-K_{S_{8}}+E_{1}$, we get two further section $\eta_{1} \kappa_{1}, \eta_{1} \kappa_{2}$. As the previous quadratic relations do not induce relations in this degree of $\operatorname{Cox}\left(S_{8}\right)$, and because we calculate $\operatorname{dim} H^{0}\left(S_{8}, D\right)=3$ for this nef degree, we obtain 27 relations, which can be calculated explicitly as before.

Every $(-1)$-curve has intersection number 2 with exactly 56 other curves (e.g. $\left(E_{1}, D\right)=2$ if and only if $D \in\left\{C_{1, i}, V_{1, i, j}, F_{i, j}, T_{i}\right\}$ for $i, j \neq 1$ ), so it occurs in exactly 56 (2)-rulings. On the other hand, as every (2)-ruling can be written in 28 ways as the sum of two $(-1)$-curves, the total number of (2)-rulings is $\frac{240 \cdot 56}{2 \cdot 28}=240$. Therefore, we obtain another 6480 relations from the (2)-rulings. To determine them explicitly, we need the following more detailed information:

LEMMA 3.9. Each of the 240 (2)-rulings can be written as a sum of two $(-1)$-curves in the following 28 ways:

- 8 (2)-rulings of the form

$$
\begin{gathered}
-K_{S_{8}}+E_{i}=3 H-\left(E_{1}+\cdots+E_{8}\right)+E_{i}: \\
\left\{E_{j}+C_{j, i}, m_{j, k}+Q_{i, j, k} \mid j, k \neq i\right\} .
\end{gathered}
$$

- $\left(\begin{array}{l}8 \\ 2\end{array}\right)=28$ (2)-rulings of the form

$$
-K_{S_{8}}+m_{i, j}=4 H-\left(E_{1}+\cdots+E_{8}\right)-\left(E_{i}+E_{j}\right):
$$

$\left\{\begin{array}{l|l}E_{k}+V_{i, j, k}, m_{i, k}+C_{j, k}, & k \notin\{i, j\}, \\ m_{j, k}+C_{i, k}, Q_{a, b, c}+Q_{d, e, f} & \{i, j, a, b, c, d, e, f\}=\{1, \ldots, 8\}\end{array}\right\}$.

- $\left(\begin{array}{l}8 \\ 3\end{array}\right)=56$ (2)-rulings of the form

$$
-K_{S_{8}}+Q_{i, j, k}=5 H-2\left(E_{1}+\cdots+E_{8}\right)+E_{i}+E_{j}+E_{k}:
$$

$\left\{\begin{array}{l|l}E_{i}+F_{j, k}, E_{j}+F_{i, k}, E_{k}+F_{i, j}, m_{a, b}+V_{c, d, e}, & \left\{\begin{array}{l}\{i, j, k, a, b, c, d, e\} \\ Q_{i, j, l}+C_{l, k}, Q_{i, k, l}+C_{l, j}, Q_{j, k, l}+C_{l, i}\end{array}\right. \\ =\{1, \ldots, 8\}, l \notin\{i, j, k\}\end{array}\right\}$. 
- $8 \cdot 7=56(2)$-rulings of the form

$$
\begin{aligned}
& -K_{S_{8}}+C_{i, j}=6 H-2\left(E_{1}+\cdots+E_{8}\right)-E_{i}+E_{j}: \\
& \left\{E_{j}+T_{i}, m_{i, k}+F_{j, k}, Q_{j, k, l}+V_{i, k, l}, C_{i, k}+C_{k, j} \mid k, l \notin\{i, j\}\right\} \text {. } \\
& \text { - }\left(\begin{array}{l}
8 \\
3
\end{array}\right)=56(2) \text {-rulings of the form } \\
& -K_{S_{8}}+V_{i, j, k}=7 H-2\left(E_{1}+\cdots+E_{8}\right)-\left(E_{i}+E_{j}+E_{k}\right): \\
& \left\{\begin{array}{l}
T_{i}+m_{j, k}, S_{j}+m_{i, k}, S_{k}+m_{i, j}, F_{a, b}+Q_{c, d, e}, \\
V_{i, j, l}+C_{k, l}, V_{i, k, l}+C_{j, l}, V_{j, k, l}+C_{i, l}
\end{array} \mid=\left\{\begin{array}{r}
\{i, j, k, a, b, c, d, e\} \\
\{1, \ldots, 8\}, l \notin\{i, j, k\}
\end{array}\right\} .\right. \\
& \text { - }\left(\begin{array}{l}
8 \\
2
\end{array}\right)=28(2) \text {-rulings of the form } \\
& -K_{S_{8}}+F_{i, j}=8 H-3\left(E_{1}+\cdots+E_{8}\right)+E_{i}+E_{j}: \\
& \left\{\begin{array}{l|l}
S_{k}+Q_{i, j, k}, F_{i, k}+C_{k, j}, & k \notin\{i, j\}, \\
F_{j, k}+C_{k, i}, V_{a, b, c}+V_{d, e, f} & \{i, j, a, b, c, d, e, f\}=\{1, \ldots, 8\}
\end{array}\right\} . \\
& -K_{S_{8}}+T_{i}=9 H-3\left(E_{1}+\cdots+E_{8}\right)-E_{i}: \\
& \left\{S_{j}+C_{i, j}, F_{j, k}+V_{i, j, k} \mid j, k \neq i\right\} .
\end{aligned}
$$

Furthermore, the 242 generators give the 123 quadratic monomials

$$
\eta_{i} \sigma_{i}, \quad \mu_{i, j} \psi_{i, j}, \quad \nu_{i, j, k} \phi_{i, j, k}, \quad \lambda_{i, j} \lambda_{j, i}, \quad \kappa_{1}^{2}, \kappa_{1} \kappa_{2}, \kappa_{2}^{2}
$$

in the 4-dimensional subspace $H^{0}\left(S_{8},-2 K_{S_{8}}\right)$ of $\operatorname{Cox}\left(S_{8}\right)$. Note that $-2 K_{S_{8}}$ is the unique (3)-ruling. As the relations coming from rulings and (2)-rulings do not induce relations in $H^{0}\left(S_{8},-2 K_{S_{8}}\right)$, we obtain another 119 relations. Their equations can be calculated in the same way as before.

Lemma 3.10. There are exactly 17399 independent quadratic relations in $\operatorname{Cox}\left(S_{8}\right)$.

Proof. The relations in $\operatorname{Cox}\left(S_{8}\right)$ are generated by relations which are homogeneous with respect to the $\operatorname{Pic}\left(S_{8}\right)$-grading. A quadratic relation involving a term $\xi\left(D_{1}\right) \xi\left(D_{2}\right)$ has degree $D=D_{1}+D_{2}$. The relations of degree $D_{1}+D_{2}$ depend on the intersection number $n=\left(D_{1}, D_{2}\right)$ :

- If $n=1$, then $D$ is a (1)-ruling. As described above, we have exactly 10800 corresponding relations.

- If $n=2$, then $D$ is a (2)-ruling. We have described the 6480 resulting relations.

- If $n=3$, then $D=-2 K_{S_{8}}$, which results in exactly 119 quadratic relations.

- If $n=0$, then $D=D_{1}+D_{2}$ is not nef since $\left(D, D_{1}\right)=-1$. However, by results of [HT04, Section 3], the relations in $\operatorname{Cox}\left(S_{8}\right)$ are generated by relations in nef degrees.

- If $n=-1$, then $D_{1}=D_{2}$, and $\left(D, D_{1}\right)=-2$, so $D$ is not nef, giving no generating relations as before.

There are no other quadratic relations involving $\kappa_{i}$ because the 240 degrees $-K_{S_{8}}+D_{1}$ for some $(-1)$-curve $D_{1}$ are exactly the (2)-rulings, and the degree $-2 K_{S_{8}}$ has also been considered. 
Let $J_{8}$ be the ideal generated by these 17399 quadratic relations in

$$
R_{8}=\mathbb{K}\left[\eta_{i}, \mu_{i, j}, \nu_{i, j, k}, \lambda_{i, j}, \phi_{i, j, k}, \psi_{i, j}, \sigma_{i}, \kappa_{i}\right] .
$$

Proof of Lemma 3.4. Let $D \in \mathcal{D}_{8} \backslash\left\{K_{1}, K_{2}\right\}$ be any (-1)-curve. We call a variable $\xi\left(D^{\prime}\right)$ for a $(-1)$-curve $D^{\prime} \in \mathcal{D}_{8}$ an $(n)$-variable if $\left(D, D^{\prime}\right)=n$.

As for $r=7$ in the previous section, we show that the rulings determine the (1)-variables in terms of the (0)-variables and $\xi(D) \neq 0$.

For the two variables $\kappa_{i}=\xi\left(K_{i}\right)$ corresponding to $-K_{S_{8}}$, we use the (2)ruling $-K_{S_{8}}+D$ : As $\left(D,-K_{S_{8}}+D\right)=0$, we have $\left(D, D_{i}\right)=\left(D, D_{i}^{\prime}\right)=0$ for any other possibility to write $-K_{S_{8}}+D$ as the sum of two $(-1)$-curves $D_{i}, D_{i}^{\prime}$. Since $\left(-K_{S_{8}}+D,-K_{S_{8}}\right)=2$, by [BP04, Proposition 3.4], the quadratic monomials $\xi\left(D_{i}\right) \xi\left(D_{i}^{\prime}\right)$ span $H^{0}\left(S_{8},-K_{S_{8}}+D\right)$, so this (2)-ruling induces relations of the form

$$
\kappa_{i} \xi(D)=\sum a_{i} \xi\left(D_{i}\right) \xi\left(D_{i}^{\prime}\right) .
$$

Therefore, $\kappa_{1}, \kappa_{2}$ are determined by $\xi(D)$ and the (0)-variables.

Any (2)-coordinate $\xi\left(D^{\prime}\right)$ is determined by the (2)-ruling $D+D^{\prime}$ : As $\left(D, D+D^{\prime}\right)=1$, we have $\left(D, D_{i}\right)=0$ and $\left(D, D_{i}^{\prime}\right)=1$ for every other possibility to write $D+D^{\prime}$ as the sum of two $(-1)$-curves $D_{i}, D_{i}^{\prime}$. Furthermore, if $D+D^{\prime}=-K_{S_{8}}+D^{\prime \prime}$, then $\left(D, D^{\prime \prime}\right)=0$. Hence, the relations corresponding to this (2)-ruling determine $\xi\left(D^{\prime}\right)$ in terms of the (0)- and (1)-variables and $\kappa_{1}, \kappa_{2}, \xi(D)$.

Finally, there is a unique (3)-coordinate $D^{\prime}$, where $D+D^{\prime}=-2 K_{S_{8}}$ is the (3)-ruling. As all other variables are known at this point, the relations corresponding to $-2 K_{S_{8}}$ containing the term $\xi(D) \xi\left(D^{\prime}\right)$ determine $\xi\left(D^{\prime}\right)$.

Consider a point in $U_{K_{j}}$, i.e., with $\kappa_{j} \neq 0$. As above, by [BP04, Proposition 3.4], $H^{0}\left(S_{8},-2 K_{S_{8}}\right)$ is spanned by the monomials $\xi\left(D_{i}\right) \xi\left(D_{i}^{\prime}\right)$ for (3)rulings $D_{i}, D_{i}^{\prime}$. Therefore, we have relations of the form

$$
\kappa_{j}^{2}=\sum a_{i} \xi\left(D_{i}\right) \xi\left(D_{i}^{\prime}\right)
$$

which shows that $\xi\left(D_{i}\right) \neq 0$ for some $i$. This proves that $Z_{8} \backslash\{0\}$ is covered by the sets $U_{D}$ for $D \in \mathcal{D}_{8} \backslash\left\{K_{1}, K_{2}\right\}$. 


\section{CHAPTER 4}

\section{Universal torsors and homogeneous spaces}

\subsection{Introduction}

In this chapter, we continue our investigations of universal torsors over smooth Del Pezzo surfaces over an algebraically closed field $\mathbb{K}$ of characteristic 0. The results first appeared in [Der06d.

The blow-up of $\mathbb{P}^{2}$ in $r$ points in general position is a smooth Del Pezzo surface $S_{r}$ of degree $9-r$; we will assume that $r \in\{3, \ldots, 7\}$. The Picard group $\operatorname{Pic}\left(S_{r}\right)$ is a lattice with the non-degenerate intersection form $(\cdot, \cdot)$. As explained in Section 1.4 $\operatorname{Pic}\left(S_{r}\right)$ contains a canonical root system $\Phi_{r}$, which carries the action of the associated Weyl group $W_{r}$, see Table 4.1 .

\begin{tabular}{|c||c|c|c|c|c|}
\hline$r$ & 3 & 4 & 5 & 6 & 7 \\
\hline \hline$\Phi_{r}$ & $\mathbf{A}_{2}+\mathbf{A}_{1}$ & $\mathbf{A}_{4}$ & $\mathbf{D}_{5}$ & $\mathbf{E}_{6}$ & $\mathbf{E}_{7}$ \\
\hline$N_{r}$ & 6 & 10 & 16 & 27 & 56 \\
\hline
\end{tabular}

TABle 4.1. The root systems associated to Del Pezzo surfaces.

It was a general expectation that the Weyl group symmetry on $\operatorname{Pic}\left(S_{r}\right)$ should be a reflection of a geometric link between Del Pezzo surfaces and algebraic groups. Here we show that universal torsors of smooth Del Pezzo surfaces of degree 2 and 3 admit an embedding into a certain flag variety for the corresponding algebraic group. The degree 5 case goes back to Salberger (talk at the Borel seminar Bern, June 1993) following Mumford [MS72, and independently Skorobogatov [Sko93]. The degree 4 case was treated in the thesis of Popov [Pop01, Chapter 6]. The existence of such an embedding in general was conjectured by Batyrev in his lecture at the conference Diophantine geometry (Universität Göttingen, June 2004). Skorobogatov announced related work in progress (joint with Serganova) at the conference Cohomological approaches to rational points (MSRI, March 2006).

By [BP04, Section 2], the simple roots of $\Phi_{r-1}$ (with $\Phi_{2}=\mathbf{A}_{2}$ ) can be identified with a subset $I_{r}$ of the simple roots of $\Phi_{r}$ whose complement consists of exactly one simple root $\alpha_{r}$, with associated fundamental weight $\varpi_{r}$. The Weyl group $W_{r}$ acts on the weight lattice of the linear algebraic group $G_{r}$ associated to $\Phi_{r}$. The fundamental representation $\varrho_{r}$ of $G_{r}$ with highest weight $\varpi_{r}$ has dimension $N_{r}$ as listed in Table 4.1 the weights of $\varrho_{r}$ can be identified with classes of $(-1)$-curves $E \subset S_{r}$.

Let $P_{r}$ be the maximal parabolic subgroup corresponding to $I_{r}$, and let $H_{r}$ be the affine cone over $G_{r} / P_{r}$. It is well-known that $H_{r}$ is given by quadratic equations in affine space $\mathbb{A}^{N_{r}}$. For $r=6$, the equations are all partial 
derivatives of a certain cubic form on the 27-dimensional representation $\varrho_{6}$ of $G_{6}$. For $r=7$, equations can be found in Fre53. In both cases, the equations were already known to E. Cartan in the 19th century.

The universal torsor $\mathcal{T}_{S_{r}}$ over $S_{r}$ (Definition 2.2) is a $T_{\mathrm{NS}}\left(S_{r}\right)$-bundle over $S_{r}$, where $T_{\mathrm{NS}}\left(S_{r}\right)$ is the Néron-Severi torus of $S_{r}$ (Section 2.2).

The Cox ring (Definition 2.4 of $S_{r}$ is naturally graded by $\operatorname{Pic}\left(S_{r}\right)$. It is generated by $N_{r}$ sections $\xi(E)$ corresponding to the (-1)-curves $E$ on $S_{r}$. The ideal of relations (up to radical) in $\operatorname{Cox}\left(S_{r}\right)$ is generated by certain quadratic relations which are homogeneous with respect to the $\mathrm{Pic}\left(S_{r}\right)$-grading (see Chapter 3 for more details). Let $\mathbb{A}\left(S_{r}\right):=\operatorname{Spec}\left(\operatorname{Cox}\left(S_{r}\right)\right) \subset \mathbb{A}^{N_{r}}$ be the corresponding affine variety. The universal torsor $\mathcal{T}_{S_{r}}$ is an open subset of $\mathbb{A}\left(S_{r}\right)$ (cf. [HK00]).

We have seen that both $\mathbb{A}\left(S_{r}\right)$ and $H_{r}$ can be viewed as embedded into $\mathbb{A}^{N_{r}}$, with a natural identification of the coordinates. For the embedding of $\mathbb{A}\left(S_{r}\right)$, we have some freedom: As the generators of $\operatorname{Cox}\left(S_{r}\right)$ are canonical only up to a non-zero constant, we can choose a rescaling factor for each of the $N_{r}$ coordinates, giving a $N_{r}$-parameter family of embeddings of $\mathbb{A}\left(S_{r}\right)$ into affine space. The task is to find a rescaling such that $\mathbb{A}\left(S_{r}\right)$ is embedded into $H_{r}$.

More precisely, we start with an arbitrary embedding

$$
\mathbb{A}\left(S_{r}\right) \subset \mathbb{A}_{r}:=\operatorname{Spec}\left(\mathbb{K}\left[\xi(E) \mid E \text { is a }(-1) \text {-curve on } S_{r}\right]\right) \cong \mathbb{A}^{N_{r}},
$$

and view

$$
H_{r} \subset \mathbb{A}_{r}^{\prime}:=\operatorname{Spec}\left(\mathbb{K}\left[\xi^{\prime}(E) \mid E \text { is a }(-1) \text {-curve on } S_{r}\right]\right) \cong \mathbb{A}^{N_{r}}
$$

as embedded into a different affine space. An isomorphism $\phi_{r}: \mathbb{A}_{r} \rightarrow \mathbb{A}_{r}^{\prime}$ such that

$$
\phi_{r}^{*}\left(\xi^{\prime}(E)\right)=\xi^{\prime \prime}(E) \cdot \xi(E)
$$

for each of the $N_{r}$ coordinates, with $\xi^{\prime \prime}(E) \in \mathbb{K}^{*}:=\mathbb{K} \backslash\{0\}$, is called a rescaling, and the factors $\xi^{\prime \prime}(E)$ are (a system of) rescaling factors. A rescaling $\phi_{r}$ which embeds $\mathbb{A}\left(S_{r}\right)$ into $H_{r}$ is called a good rescaling.

Our main result is:

Theorem 4.1. Let $S_{r}$ be a smooth Del Pezzo surface of degree $9-r$ and $\mathbb{A}\left(S_{r}\right)$ the affine variety described above. Let $H_{r}$ be the affine cone over the flag variety $G_{r} / P_{r}$ associated to the root system $\Phi_{r}$ as in Table 4.1.

For $r \in\{6,7\}$, there exists a $\left(N_{r-2}+2\right)$-parameter family of good rescalings $\phi_{r}$ which embed $\mathbb{A}\left(S_{r}\right)$ into $H_{r}$.

REMARK 4.2. The rescaling factors are naturally graded by $\operatorname{Pic}\left(S_{r}\right) \cong$ $\mathbb{Z}^{r+1}$, and we will see in Section 4.3 that the conditions for good rescaling are homogeneous with respect to this grading. Therefore, for each good rescaling $\phi_{r}$, there is a $(r+1)$-parameter family of good rescalings which differ from $\phi_{r}$ only by the action of $T_{\mathrm{NS}}\left(S_{r}\right) \cong \mathbb{G}_{\mathrm{m}}^{r+1}$. Similarly, $T_{\mathrm{NS}}\left(S_{r}\right)$ acts on $\mathbb{A}\left(S_{r}\right)$ (Remark 2.11), and it is easy to see that the image of $\mathbb{A}\left(S_{r}\right)$ in $H_{r}$ is the same for all good rescalings in the same $(r+1)$-parameter family. Consequently, the $\left(N_{r-2}+2\right)$-parameter family of good rescalings gives rise to a $\left(N_{r-2}-r+1\right)$-parameter family of images of $\mathbb{A}\left(S_{r}\right)$ in $H_{r}$. 
For $r=5$, we have $N_{r-2}-r+1=2$, and by Pop01, Section 6.3], there is a two-parameter family of images of $\mathbb{A}\left(S_{5}\right)$ under good rescalings in $H_{5}$.

In Section 4.2, we recall the classical equations for the homogeneous spaces $G_{r} / P_{r}$ and give a simplified description on a certain Zariski open subset; this will help to find good rescalings. In Section 4.3, we derive conditions on the rescaling factors in terms of the description of $\operatorname{Cox}\left(S_{r}\right)$ and $G_{r} / P_{r}$. In Section 4.4 and Section 4.5 , we determine good rescalings in degree 3 and 2, finishing the proof of Theorem 4.1 .

\subsection{Homogeneous spaces}

In this section, we examine the equations defining the affine cone $H_{r} \subset$ $\mathbb{A}_{r}^{\prime}$ over $G_{r} / P_{r}$ for $r \in\{6,7\}$. For the $N_{r}$ coordinates $\xi^{\prime}(E)$ of $\mathbb{A}_{r}^{\prime}$, we also use the names $\eta_{i}^{\prime}, \mu_{i, j}^{\prime}, \lambda_{i}^{\prime}$, and furthermore $\nu_{i, j}^{\prime}$ in the case $r=7$.

These coordinates are in obvious correspondence to the coordinates of $\mathbb{A}_{r}$, for which we use the names $\eta_{i}, \mu_{i, j}, \lambda_{i}$ (see Section 3.3), and furthermore $\nu_{i, j}$ in the case $r=7$ (see Section 3.4) as for the generators of $\operatorname{Cox}\left(S_{r}\right)$.

In particular, we show that $H_{r}$ is a complete intersection on the open subset $U_{r}$ of $\mathbb{A}_{r}^{\prime}$ where the coordinates $\eta_{1}^{\prime}, \ldots, \eta_{r}^{\prime}$ are non-zero.

We will see that $H_{r}$ is defined by quadratic relations which are homogeneous with respect to the $\operatorname{Pic}\left(S_{r}\right)$-grading. For each (1)-ruling $D$, we have exactly one relation $p_{D}$ of degree $D$, and furthermore in the case $r=7$, we have eight relations $p_{-K_{S_{7}}}^{(1)}, \ldots, p_{-K_{S_{7}}}^{(8)}$ where we use the convention $p_{-K_{S_{7}}}:=p_{-K_{S_{7}}}^{(1)}$. For any possibility to write $D$ as the sum of two $(-1)$-curves $E, E^{\prime}$, the relation $p_{D}$ has a term $\xi^{\prime}(E) \xi^{\prime}\left(E^{\prime}\right)$ with a non-zero coefficient.

Definition 4.3. For a $(-1)$-curve $E$, let $U_{E}$ be the open subset of $\mathbb{A}_{r}^{\prime}$ where $\xi^{\prime}(E)$ is non-zero. Let $\mathcal{N}(E)_{k}$ be the set of $(-1)$-curves $E^{\prime}$ with $\left(E, E^{\prime}\right)=k$, and let $\Xi^{\prime}(E)_{k}$ be the set of the corresponding $\xi^{\prime}\left(E^{\prime}\right)$. Let $\mathcal{N}(E)_{>k}$ and $\Xi^{\prime}(E)_{>k}$ be defined similarly, but with the condition $\left(E, E^{\prime}\right)>$ $k$.

Let $U_{r} \subset \mathbb{A}_{r}^{\prime}$ be the intersection of $U_{E_{1}}, \ldots, U_{E_{r}}$.

Note that $\mathcal{N}(E)_{0}$ has exactly $N_{r-1}$ elements because we can identify its elements with the $(-1)$-curves on $S_{r-1}$. Since the only $(-1)$-curve intersecting $E$ negatively is $E$ itself, the number of elements of $\mathcal{N}(E)_{>0}$ is $N_{r}-N_{r-1}-1$.

Proposition 4.4. Let

$$
\Psi_{r}: H_{r} \cap U_{r} \rightarrow U_{r-1} \times\left(\mathbb{A}^{1} \backslash\{0\}\right)
$$

be the projection to the coordinates $\xi^{\prime}(E) \in \Xi^{\prime}\left(E_{1}\right)_{0}$ and $\eta_{1}^{\prime}$. The map $\Psi_{r}$ is an isomorphism. The dimension of $H_{r}$ is $N_{r-1}+1$.

Proof. If $D=E_{1}+E$ is a (1)-ruling, then $\left(E_{1}, D\right)=0$, and all variables occurring in $p_{D}$ besides $\eta_{1}^{\prime}$ and $\xi^{\prime}(E)$ are elements of $\Xi^{\prime}\left(E_{1}\right)_{0}$. For $\eta_{1}^{\prime} \neq 0$, the relation $p_{D}$ expresses $\xi^{\prime}(E)$ in terms of $\eta_{1}^{\prime}$ and $\Xi^{\prime}\left(E_{1}\right)_{0}$.

For a (2)-ruling $D=E_{1}+E$, we have $\left(E_{1}, D\right)=1$, so the relation $p_{D}$ expresses $\xi^{\prime}(E)$ in terms of $\eta_{1}^{\prime}$ and monomials $\xi^{\prime}\left(E_{i}^{\prime}\right) \xi^{\prime}\left(E_{i}^{\prime \prime}\right)$ where $\xi\left(E_{i}^{\prime}\right) \in$ 
$\Xi^{\prime}\left(E_{1}\right)_{0}$ and $\xi^{\prime}\left(E_{i}^{\prime \prime}\right) \in \Xi^{\prime}\left(E_{1}\right)_{1}$. Using the expressions for the elements of $\Xi^{\prime}\left(E_{1}\right)_{1}$ of the first step, this shows that we can express the coordinates $\Xi^{\prime}\left(E_{1}\right)_{>0}$ in terms of $\eta_{1}^{\prime}$ and $\Xi^{\prime}\left(E_{1}\right)_{0}$ by using the $N_{r}-N_{r-1}-1$ relations $g_{E_{1}+E}$ for $E \in \mathcal{N}(E)_{>0}$. This allows us to construct a map

$$
\Psi_{r}^{\prime}: U_{r-1} \times\left(\mathbb{A}^{1} \backslash\{0\}\right) \rightarrow \mathbb{A}_{r}^{\prime} .
$$

It remains to show that the image of $\Psi_{r}^{\prime}$ is in $H_{r}$, i.e., that the resulting point also satisfies the remaining equations which define $H_{r}$. This is done in Lemma 4.6 and Lemma 4.7 below.

REMARK 4.5. Proposition 4.4 is also true if we enlarge $U_{r}$ to $U_{E_{1}}$ and $U_{r-1}$ to $\mathbb{A}_{r-1}^{\prime}$. However, the proofs of Lemma 4.6 and Lemma 4.7 are slightly simplified by restricting to $U_{r}$.

First, we consider the case $r=6$. It is known Pop01, Section 2.2.2] that $H_{6} \subset \mathbb{A}_{6}$ is given by the 27 partial derivatives of

$$
F\left(M_{1}, M_{2}, M_{3}\right):=\operatorname{det} M_{1}+\operatorname{det} M_{2}+\operatorname{det} M_{3}-\operatorname{tr}\left(M_{1} M_{2} M_{3}\right),
$$

where

$$
M_{1}:=\left(\begin{array}{ccc}
\eta_{1}^{\prime} & \lambda_{1}^{\prime} & \mu_{2,3}^{\prime} \\
\eta_{2}^{\prime} & \lambda_{2}^{\prime} & \mu_{1,3}^{\prime} \\
\eta_{3}^{\prime} & \lambda_{3}^{\prime} & \mu_{1,2}^{\prime}
\end{array}\right), \quad M_{2}:=\left(\begin{array}{ccc}
\lambda_{4}^{\prime} & \lambda_{5}^{\prime} & \lambda_{6}^{\prime} \\
\eta_{4}^{\prime} & \eta_{5}^{\prime} & \eta_{6}^{\prime} \\
\mu_{5,6}^{\prime} & \mu_{4,6}^{\prime} & \mu_{4,5}^{\prime}
\end{array}\right)
$$

and

$$
M_{3}:=\left(\begin{array}{lll}
\mu_{1,4}^{\prime} & \mu_{2,4}^{\prime} & \mu_{3,4}^{\prime} \\
\mu_{1,5}^{\prime} & \mu_{2,5}^{\prime} & \mu_{3,5}^{\prime} \\
\mu_{1,6}^{\prime} & \mu_{2,6}^{\prime} & \mu_{3,6}^{\prime}
\end{array}\right)
$$

Note that the terms of $\operatorname{tr}\left(M_{1} M_{2} M_{3}\right)$ are $M_{1}^{(i, j)} M_{2}^{(j, k)} M_{3}^{(k, i)}$ for $i, j, k \in$ $\{1,2,3\}$ (where $M_{a}^{(b, c)}$ is the entry $(b, c)$ of the matrix $M_{a}$ ), so the number of terms of $F$ is $3 \cdot 6+3^{3}=45$. Each is a product of three variables $\xi^{\prime}(E), \xi^{\prime}\left(E^{\prime}\right), \xi^{\prime}\left(E^{\prime \prime}\right)$ such that the corresponding $(-1)$-curves $E, E^{\prime}, E^{\prime \prime}$ on $S_{6}$ form a triangle, and their divisor classes add up to $-K_{S_{6}}$. The coefficient is +1 in the nine cases

$$
\begin{array}{ccc}
\eta_{1}^{\prime} \mu_{1,2}^{\prime} \lambda_{2}^{\prime}, & \eta_{2}^{\prime} \mu_{2,3}^{\prime} \lambda_{3}^{\prime}, & \eta_{3}^{\prime} \mu_{1,3}^{\prime} \lambda_{1}^{\prime}, \\
\eta_{4}^{\prime} \mu_{4,6}^{\prime} \lambda_{6}^{\prime}, & \eta_{5}^{\prime} \mu_{4,5}^{\prime} \lambda_{4}^{\prime}, & \eta_{6}^{\prime} \mu_{5,6}^{\prime} \lambda_{5}^{\prime}, \\
\mu_{1,4}^{\prime} \mu_{2,5}^{\prime} \mu_{3,6}^{\prime}, & \mu_{1,5}^{\prime} \mu_{2,6}^{\prime} \mu_{3,4}^{\prime}, & \mu_{1,6}^{\prime} \mu_{2,4}^{\prime} \mu_{3,5}^{\prime}
\end{array}
$$

and -1 in the remaining 36 cases. (Of course, there is some choice here, for example by permuting the indices $1, \ldots, 6$, but it is not as simple as choosing any 9 of the 45 terms to have the coefficient +1 . See [Lur01, Section 5] for more details.)

The derivative with respect to $\xi^{\prime}(E)$ contains five terms $\pm \xi^{\prime}\left(E^{\prime}\right) \xi^{\prime}\left(E^{\prime \prime}\right)$ corresponding to the five ways to write the (1)-ruling $D:=-K_{S_{6}}-E$ as the sum of two intersecting $(-1)$-curves $E^{\prime}, E^{\prime \prime}$. We will denote it by $p_{D}=p_{-K_{S_{6}}-E}$.

LEMMA 4.6. For $\eta_{1}^{\prime} \neq 0$ and any values of

$$
\Xi^{\prime}\left(E_{1}\right)_{0}=\left\{\eta_{2}^{\prime}, \ldots, \eta_{6}^{\prime}, \mu_{2,3}^{\prime}, \ldots, \mu_{5,6}^{\prime}, \lambda_{1}^{\prime}\right\}
$$

with non-zero $\eta_{2}^{\prime}, \ldots, \eta_{6}^{\prime}$, the equations $p_{E_{1}+E}$ for

$$
E \in \mathcal{N}\left(E_{1}\right)_{1}=\left\{m_{1,2}, \ldots, m_{1,6}, Q_{2}, \ldots, Q_{6}\right\}
$$


define a point of $H_{6}$.

Proof. As $T_{\mathrm{NS}}\left(S_{6}\right)$ acts on $H_{6}$ and $\left\{E_{1}, \ldots, E_{6}\right\}$ is a subset of a basis of $\operatorname{Pic}\left(S_{6}\right)$, we may assume that $\eta_{1}^{\prime}=\cdots=\eta_{6}^{\prime}=1$. Then for $i \in\{2, \ldots, 6\}$, the equation $p_{E_{1}+m_{1, i}}$ allows us to express $\mu_{1, i}^{\prime}$ in terms of the remaining $\mu_{i, j}^{\prime}$ :

$$
\begin{aligned}
& \mu_{1,2}^{\prime}=\mu_{2,3}^{\prime}+\mu_{2,4}^{\prime}+\mu_{2,5}^{\prime}+\mu_{2,6}^{\prime}, \quad \mu_{1,3}^{\prime}=\mu_{2,3}^{\prime}-\mu_{3,4}^{\prime}-\mu_{3,5}^{\prime}-\mu_{3,6}^{\prime}, \\
& \mu_{1,4}^{\prime}=-\mu_{2,4}^{\prime}-\mu_{3,4}^{\prime}+\mu_{4,5}^{\prime}-\mu_{4,6}^{\prime}, \quad \mu_{1,5}^{\prime}=-\mu_{2,5}^{\prime}-\mu_{3,5}^{\prime}-\mu_{4,5}^{\prime}+\mu_{5,6}^{\prime} \\
& \mu_{1,6}^{\prime}=-\mu_{2,6}^{\prime}-\mu_{3,6}^{\prime}+\mu_{4,6}^{\prime}-\mu_{5,6}^{\prime}
\end{aligned}
$$

Furthermore, for $i \in\{2, \ldots, 6\}$, we can use $p_{E_{1}+Q_{i}}$ in order to express $\lambda_{i}^{\prime}$ in terms of $\lambda_{1}^{\prime}$ and $\mu_{j, k}^{\prime}$ :

$$
\begin{aligned}
& \lambda_{2}^{\prime}=\mu_{3,4}^{\prime} \mu_{5,6}^{\prime}+\mu_{3,5}^{\prime} \mu_{4,6}^{\prime}+\mu_{3,6}^{\prime} \mu_{4,5}^{\prime}+\lambda_{1}^{\prime} \\
& \lambda_{3}^{\prime}=-\mu_{2,4}^{\prime} \mu_{5,6}^{\prime}-\mu_{2,5}^{\prime} \mu_{4,6}^{\prime}-\mu_{2,6}^{\prime} \mu_{4,5}^{\prime}+\lambda_{1}^{\prime} \\
& \lambda_{4}^{\prime}=-\mu_{2,3}^{\prime} \mu_{5,6}^{\prime}+\mu_{2,5}^{\prime} \mu_{3,6}^{\prime}-\mu_{2,6}^{\prime} \mu_{3,5}^{\prime}-\lambda_{1}^{\prime} \\
& \lambda_{5}^{\prime}=-\mu_{2,3}^{\prime} \mu_{4,6}^{\prime}-\mu_{2,4}^{\prime} \mu_{3,6}^{\prime}+\mu_{2,6}^{\prime} \mu_{3,4}^{\prime}-\lambda_{1}^{\prime} \\
& \lambda_{6}^{\prime}=-\mu_{2,3}^{\prime} \mu_{4,5}^{\prime}+\mu_{2,4}^{\prime} \mu_{3,5}^{\prime}-\mu_{2,5}^{\prime} \mu_{3,4}^{\prime}-\lambda_{1}^{\prime}
\end{aligned}
$$

By substituting and expanding, we check that the remaining 17 relations are fulfilled. Therefore, the resulting point lies in $H_{6}$.

Next, we obtain similar results in the case $r=7$. The $N_{7}=56$ coordinates $\xi^{\prime}(E)$ in $\mathbb{A}_{r}^{\prime}$ are $\eta_{i}^{\prime}, \mu_{j, k}^{\prime}, \nu_{j, k}^{\prime}, \lambda_{i}^{\prime}$ for $i, j, k \in\{1, \ldots, 7\}$ and $j<k$. The equations for $H_{7}$ are described in Fre53] in terms of 56 coordinates $x^{i j}, y_{i j}$ $(i<j \in\{1, \ldots, 8\})$. They correspond to our generators as follows:

$$
\eta_{i}^{\prime}=x^{i 8}, \quad \mu_{k, l}^{\prime}=y_{k l}, \quad \nu_{k, l}^{\prime}=x^{k l}, \quad \lambda_{i}^{\prime}=y_{i 8} .
$$

For the (1)-rulings $D$ (see Lemma 3.7), the relations $p_{D}$ are $u^{i j k l}$ and $v_{j}^{i}$ as below. In the first column of Table 4.2 , we list a symbol $D_{I}^{(n)}$ assigned to the (1)-ruling in the second column, and the third column gives the corresponding relation.

\begin{tabular}{|c||c|c|}
\hline symbol & $(1)$-ruling $D=D_{I}^{(n)}$ & relation $p_{D}$ \\
\hline \hline$D_{i}^{(1)}$ & $H-E_{i}$ & $v_{i}^{8}$ \\
$D_{i, j, k}^{(2)}$ & $2 H-\left(E_{1}+\cdots+E_{7}\right)+E_{i}+E_{j}+E_{k}$ & $u^{i j k 8}$ \\
$D_{i, j}^{(3)}$ & $3 H-\left(E_{1}+\cdots+E_{7}\right)+E_{i}-E_{j}$ & $v_{j}^{i}$ \\
$D_{i, j, k, l}^{(4)}$ & $4 H-2\left(E_{1}+\cdots+E_{7}\right)+E_{i}+E_{j}+E_{k}+E_{l}$ & $u^{i j k l}$ \\
$D_{i}^{(5)}$ & $5 H-2\left(E_{1}+\cdots+E_{7}\right)+E_{i}$ & $v_{8}^{i}$ \\
\hline
\end{tabular}

TABLE 4.2. Rulings and relations defining $G_{7} / P_{7}$.

Let

$$
u^{i j k l}=x^{i j} x^{k l}-x^{i k} x^{j l}+x^{i l} x^{j k}+\sigma \cdot\left(y_{a b} y_{c d}-y_{a c} y_{b d}+y_{a d} y_{b c}\right),
$$


where $i<j<k<l$ and $a<b<c<d$, with $(i, j, k, l, a, b, c, d)$ a permutation of $(1, \ldots, 8)$, and $\sigma$ its sign. For $i \neq j$

$$
v_{j}^{i}=\sum_{k \in(\{1, \ldots, 8\} \backslash\{i, j\})} x^{i k} y_{k j},
$$

where $x^{b a}=-x^{a b}$ and $y_{b a}=-y_{a b}$ if $b>a$.

For the (2)-ruling $-K_{S_{7}}$, we have the following eight equations with 28 terms:

$$
p_{-K_{S_{7}}}^{(i)}:=v_{i}^{i}:=-\frac{3}{4} \sum_{j \in(\{1, \ldots, 8\} \backslash\{i\})} x^{i j} y_{i j}+\frac{1}{4} \sum_{j<k \in(\{1, \ldots, 8\} \backslash\{i\})} x^{j k} y_{j k}
$$

Lemma 4.7. For $\eta_{1}^{\prime}, \ldots, \eta_{7}^{\prime} \neq 0$, the 28 coordinates

$$
\eta_{i}^{\prime} \quad(i \in\{1, \ldots, 7\}), \quad \mu_{j, k}^{\prime} \quad(j<k \in\{2, \ldots, 7\}), \quad \nu_{1, l}^{\prime} \quad(l \in\{2, \ldots, 7\})
$$

in $\Xi^{\prime}\left(E_{1}\right)_{0}$ and the 28 equations $p_{D}$ for

$$
D \in\left\{D_{2}^{(1)}, \ldots, D_{7}^{(1)}, D_{1,2,3}^{(2)}, \ldots, D_{1,6,7}^{(2)}, D_{1,2}^{(3)}, \ldots, D_{1,7}^{(3)},-K_{S_{7}}\right\}
$$

define

$$
\mu_{1, i}^{\prime} \quad(i \in\{2, \ldots, 7\}), \quad \nu_{j, k}^{\prime} \quad(j<k \in\{2, \ldots, 7\}), \quad \lambda_{l}^{\prime} \quad(l \in\{1, \ldots, 7\}),
$$

resulting in a point on $\mathrm{H}_{7}$.

Furthermore, we may replace $p_{-K_{S_{7}}}$ by $p_{D}$ for $D=D_{2,1}^{(3)}$.

Proof. As above, we may assume that $\eta_{1}^{\prime}=\cdots=\eta_{7}^{\prime}=1$ because of the action of $T_{\mathrm{NS}}\left(S_{7}\right)$. For the $27(-1)$-curves $E \in \mathcal{N}\left(E_{1}\right)_{1}$, the equation $p_{E_{1}+E}$ defines $\xi^{\prime}(E)$ directly in terms of the 28 variables in $\Xi^{\prime}\left(E_{1}\right)_{0}$; we do not list the expressions here. By substituting these results, we use $v_{1}^{1}$ in order to express $\lambda_{1}^{\prime}$ in terms of these variables:

$$
\begin{aligned}
\lambda_{1}^{\prime}= & -\mu_{2,3}^{\prime} \mu_{4,5}^{\prime} \mu_{6,7}^{\prime}+\mu_{2,3}^{\prime} \mu_{4,6}^{\prime} \mu_{5,7}^{\prime}-\mu_{2,3}^{\prime} \mu_{4,7}^{\prime} \mu_{5,6}^{\prime}+\mu_{2,4}^{\prime} \mu_{3,5}^{\prime} \mu_{6,7}^{\prime}-\mu_{2,4}^{\prime} \mu_{3,6}^{\prime} \mu_{5,7}^{\prime} \\
& +\mu_{2,4}^{\prime} \mu_{3,7}^{\prime} \mu_{5,6}^{\prime}-\mu_{2,5}^{\prime} \mu_{3,4}^{\prime} \mu_{6,7}^{\prime}+\mu_{2,5}^{\prime} \mu_{3,6}^{\prime} \mu_{4,7}^{\prime}-\mu_{2,5}^{\prime} \mu_{3,7}^{\prime} \mu_{4,6}^{\prime}+\mu_{2,6}^{\prime} \mu_{3,4}^{\prime} \mu_{5,7}^{\prime} \\
& -\mu_{2,6}^{\prime} \mu_{3,5}^{\prime} \mu_{4,7}^{\prime}+\mu_{2,6}^{\prime} \mu_{3,7}^{\prime} \mu_{4,5}^{\prime}-\mu_{2,7}^{\prime} \mu_{3,4}^{\prime} \mu_{5,6}^{\prime}+\mu_{2,7}^{\prime} \mu_{3,5}^{\prime} \mu_{4,6}^{\prime}-\mu_{2,7}^{\prime} \mu_{3,6}^{\prime} \mu_{4,5}^{\prime} \\
& -\mu_{2,3}^{\prime} \lambda_{2}^{\prime}+\mu_{2,3}^{\prime} \lambda_{3}^{\prime}-\mu_{2,4}^{\prime} \lambda_{2}^{\prime}+\mu_{2,4}^{\prime} \lambda_{4}^{\prime}-\mu_{2,5}^{\prime} \lambda_{2}^{\prime}+\mu_{2,5}^{\prime} \lambda_{5}^{\prime} \\
& -\mu_{2,6}^{\prime} \lambda_{2}^{\prime}+\mu_{2,6}^{\prime} \lambda_{6}^{\prime}-\mu_{2,7}^{\prime} \lambda_{2}^{\prime}+\mu_{2,7}^{\prime} \lambda_{7}^{\prime}-\mu_{3,4}^{\prime} \lambda_{3}^{\prime}+\mu_{3,4}^{\prime} \lambda_{4}^{\prime} \\
& -\mu_{3,5}^{\prime} \lambda_{3}^{\prime}+\mu_{3,5}^{\prime} \lambda_{5}^{\prime}-\mu_{3,6}^{\prime} \lambda_{3}^{\prime}+\mu_{3,6}^{\prime} \lambda_{6}^{\prime}-\mu_{3,7}^{\prime} \lambda_{3}^{\prime}+\mu_{3,7}^{\prime} \lambda_{7}^{\prime} \\
& -\mu_{4,5}^{\prime} \lambda_{4}^{\prime}+\mu_{4,5}^{\prime} \lambda_{5}^{\prime}-\mu_{4,6}^{\prime} \lambda_{4}^{\prime}+\mu_{4,6}^{\prime} \lambda_{6}^{\prime}-\mu_{4,7}^{\prime} \lambda_{4}^{\prime}+\mu_{4,7}^{\prime} \lambda_{7}^{\prime} \\
& -\mu_{5,6}^{\prime} \lambda_{5}^{\prime}+\mu_{5,6}^{\prime} \lambda_{6}^{\prime}-\mu_{5,7}^{\prime} \lambda_{5}^{\prime}+\mu_{5,7}^{\prime} \lambda_{7}^{\prime}-\mu_{6,7}^{\prime} \lambda_{6}^{\prime}+\mu_{6,7}^{\prime} \lambda_{7}^{\prime}
\end{aligned}
$$

We check directly by substituting and expanding that the remaining equations defining $\mathrm{H}_{7}$ are fulfilled.

As $v_{1}^{2}$ contains the term $\eta_{2}^{\prime} \lambda_{1}^{\prime}$, and $\eta_{2}^{\prime} \neq 0$, we may replace $v_{1}^{1}$ by $v_{1}^{2}$.

\subsection{Rescalings}

Let $r \in\{6,7\}$. We use the notation of Chapter 3 . We follow the strategy of the case $r=5$ Pop01, Section 6.3] in order to describe conditions for good rescalings explicitly in terms of the rescaling factors. However, we use the results of the previous section to simplify this as follows: 
Let

$$
\mathcal{M}_{6}:=\left\{E_{1}+E \mid E \in \mathcal{N}\left(E_{1}\right)_{1}\right\}
$$

and let

$$
\mathcal{M}_{7}:=\left\{E_{1}+E \mid E \in \mathcal{N}\left(E_{1}\right)_{1}\right\} \cup\left\{D_{2,1}^{(3)}\right\} .
$$

Let $\widetilde{H}_{r} \subset \mathbb{A}_{r}^{\prime}$ be the variety defined by the equations $g_{D}$ for $D \in \mathcal{M}_{r}$.

By Proposition 4.4, Lemma 4.6, and Lemma 4.7, $H_{r} \cap U_{r}=\widetilde{H}_{r} \cap U_{r}$.

REMARK 4.8. Because of $\mathcal{N}\left(E_{1}\right)_{2}=\left\{C_{1}\right\}$, it could be considered more natural to use $-K_{S_{7}}=E_{1}+C_{1}$ instead of $D_{2,1}^{(3)}=E_{2}+C_{1}$ in the definition of $\mathcal{M}_{7}$. However, we choose to avoid the (2)-ruling $-K_{S_{7}}$ for technical reasons.

Lemma 4.9. A rescaling $\phi_{r}: \mathbb{A}_{r} \rightarrow \mathbb{A}_{r}^{\prime}$ is good if and only if it embeds $\mathbb{A}\left(S_{r}\right)$ into $\widetilde{H}_{r}$.

Proof. As $H_{r} \subset \widetilde{H}_{r}$, a good rescaling $\phi_{r}$ satisfies $\phi_{r}\left(\mathbb{A}\left(S_{r}\right)\right) \subset \widetilde{H}_{r}$. Conversely, we have

$$
\phi_{r}\left(\mathbb{A}\left(S_{r}\right)\right) \cap U_{r} \subset \widetilde{H}_{r} \cap U_{r}=H_{r} \cap U_{r}
$$

by Lemma 4.6 and Lemma 4.7. Taking the closure and using that $H_{r}$ is closed and that $\mathbb{A}\left(S_{r}\right)$ is irreducible by Lemma 2.9, we conclude that $\phi_{r}\left(\mathbb{A}\left(S_{r}\right)\right) \subset H_{r}$, so the rescaling is good.

As in Theorem 3.2 , let $J_{r}$ be the ideal defining $\mathbb{A}\left(S_{r}\right)$ in $\mathbb{A}_{r}$.

In terms of the coordinate rings $\mathbb{K}\left[\mathbb{A}_{r}\right]$ and $\mathbb{K}\left[\mathbb{A}_{r}^{\prime}\right]$ and in view of the previous lemma, a rescaling $\phi_{r}$ is good if, for all $D \in \mathcal{M}_{r}$, the ideal $J_{r} \subset$ $\operatorname{rad}\left(J_{r}\right)$ contains $\phi_{r}^{*}\left(p_{D}\right)$, where $p_{D}$ is the equation defining $H_{r}$ corresponding to the (1)-ruling $D$.

As $\mathbb{K}\left[\mathbb{A}_{r}\right]$ and $\mathbb{K}\left[\mathbb{A}_{r}^{\prime}\right]$ are both graded by $\operatorname{Pic}\left(S_{r}\right)$ and $\phi_{r}^{*}$ respects this grading, we need rescaling factors such that $\phi_{r}^{*}\left(p_{D}\right)$ of degree $D \in \mathcal{M}_{r}$ is a linear combination of the equations $F_{D, 1}, \ldots, F_{D, r-3} \in J_{r}$. For concrete calculations in the next sections, we describe this more explicitly:

Let $D \in \mathcal{M}_{r}$ be a (1)-ruling, which can be written in $r-1$ ways as the sum of two $(-1)$-curves $E_{i}^{\prime}, E_{i}^{\prime \prime}$. For $i \in\{1, \ldots, r-1\}$, let

$$
\xi_{i}:=\xi\left(E_{i}^{\prime}\right) \xi\left(E_{i}^{\prime \prime}\right), \quad \xi_{i}^{\prime}:=\xi^{\prime}\left(E_{i}^{\prime}\right) \xi^{\prime}\left(E_{i}^{\prime \prime}\right), \quad \xi_{i}^{\prime \prime}:=\xi^{\prime \prime}\left(E_{i}^{\prime}\right) \xi^{\prime \prime}\left(E_{i}^{\prime \prime}\right) .
$$

Then $p_{D}$ has the form

$$
p_{D}=\sum_{i=1}^{r-1} \epsilon_{i} \xi_{i}^{\prime}
$$

with $\epsilon_{i} \in\{ \pm 1\}$.

As $\xi_{i}$ vanishes exactly on $E_{i}^{\prime} \cup E_{i}^{\prime \prime}$, the 2-dimensional space $H^{0}\left(S_{r}, \mathcal{O}(D)\right)$ is generated by any two $\xi_{i}, \xi_{i^{\prime}}$. Hence, all other $r-3$ elements $\xi_{j}$ are linear combinations of $\xi_{i}, \xi_{i^{\prime}}$, with non-vanishing coefficients. This gives $r-3$ relations of degree $D$ in $\operatorname{Cox}\left(S_{r}\right)$. Rearranging $\xi_{1}, \ldots, \xi_{r-1}$ such that the two elements $\xi_{i}, \xi_{i^{\prime}}$ of our choice have the indices $r-2$ and $r-1$, we can write them as

$$
F_{D, j}=\xi_{j}+\alpha_{j} \xi_{r-2}+\beta_{j} \xi_{r-1},
$$

for $j \in\{1, \ldots, r-3\}$, where $\alpha_{j}, \beta_{j} \in \mathbb{K}^{*}$. 
Suppose that $\phi_{r}^{*}\left(p_{D}\right)$ is a linear combination of the $F_{D, j}$ with factors $\lambda_{j}$ :

$$
\phi_{r}^{*}\left(p_{D}\right)-\sum_{j=1}^{r-3} \lambda_{j} F_{D, j}=0 .
$$

Since $\phi_{r}^{*}\left(\xi^{\prime}(E)\right)=\xi^{\prime \prime}(E) \cdot \xi(E)$, we have $\phi_{r}^{*}\left(\xi_{i}^{\prime}\right)=\xi_{i}^{\prime \prime} \cdot \xi_{i}$ for the monomials of degree 2. Then the above equation is equivalent to the vanishing of

$\sum_{i=1}^{r-3}\left(\epsilon_{i} \xi_{i}^{\prime \prime}-\lambda_{i}\right) \xi_{i}+\left(\epsilon_{r-2} \xi_{r-2}^{\prime \prime}-\sum_{j=1}^{r-3} \lambda_{j} \alpha_{j}\right) \xi_{r-2}+\left(\epsilon_{r-1} \xi_{r-1}^{\prime \prime}-\sum_{j=1}^{r-3} \lambda_{j} \beta_{j}\right) \xi_{r-1}$.

For $i \in\{1, \ldots, r-3\}$, we see by considering the coefficients of $\xi_{i}$ that we must choose $\lambda_{i}=\epsilon_{i} \xi_{i}^{\prime \prime}$. With this, consideration of the coefficients of $\xi_{r-2}$ and $\xi_{r-1}$ results in the following conditions $g_{D, 1}, g_{D, 2}$ on the rescaling factors $\xi_{j}^{\prime \prime}$, which are homogeneous of degree $D \in \operatorname{Pic}\left(S_{r}\right)$ :

$$
g_{D, 1}:=\epsilon_{r-2} \xi_{r-2}^{\prime \prime}-\sum_{j=1}^{r-3} \epsilon_{j} \alpha_{j} \xi_{j}^{\prime \prime}=0, \quad g_{D, 2}:=\epsilon_{r-1} \xi_{r-1}^{\prime \prime}-\sum_{j=1}^{r-3} \epsilon_{j} \beta_{j} \xi_{j}^{\prime \prime}=0 .
$$

Note that our choice of $\xi_{r-2}$ and $\xi_{r-1}$ in the definition of $F_{D, j}$ as discussed before 4.2 is reflected here in the sense that $g_{D, 1}$ and $g_{D, 2}$ express the corresponding $\xi_{r-2}^{\prime \prime}$ and $\xi_{r-1}^{\prime \prime}$ as linear combinations of $\xi_{1}^{\prime \prime}, \ldots, \xi_{r-3}^{\prime \prime}$ with nonzero coefficients.

This information can be summarized as follows:

Lemma 4.10. For $r \in\{6,7\}$, a rescaling is good if and only if the rescaling factors $\xi^{\prime \prime}(E)$ fulfill the equations $g_{D, 1}$ and $g_{D, 2}$ for each (1)-ruling $D \in \mathcal{M}_{r}$.

As described above precisely, the non-zero coefficients $\epsilon_{i}$ are taken from the equations $p_{D}$ (4.1) defining $H_{r}$, and the non-zero $\alpha_{j}, \beta_{j}$ are taken from the equations $F_{D, j}$ (4.2) defining $\mathbb{A}\left(S_{r}\right)$.

Let $\Xi^{\prime \prime}(E)_{k}$ (resp. $\Xi^{\prime \prime}(E)_{>k}$ ) be the set of all $\xi^{\prime \prime}\left(E^{\prime}\right)$ for $E^{\prime} \in \mathcal{N}(E)_{k}$ (resp. $\left.E^{\prime} \in \mathcal{N}(E)_{>k}\right)$. Let

$$
\Xi_{i, j}^{\prime \prime}:=\Xi^{\prime \prime}\left(E_{1}\right)_{i} \cap \Xi^{\prime \prime}\left(E_{2}\right)_{j} .
$$

We claim that we may express the rescaling factors $\Xi^{\prime \prime}\left(E_{1}\right)_{>0} \cup \Xi^{\prime \prime}\left(E_{2}\right)_{>0}$ in terms of the other $N_{r-2}+2$ rescaling factors $\left\{\eta_{1}^{\prime \prime}, \eta_{2}^{\prime \prime}\right\} \cup \Xi_{0,0}^{\prime \prime}$.

We will prove this for $r \in\{6,7\}$ as follows: The $2 \cdot\left(N_{r}-N_{r-1}-1\right)$ equations $g_{D, i}$ are homogeneous of degree $D$ with respect to the $\operatorname{Pic}\left(S_{r}\right)$ grading of the variables $\xi^{\prime \prime}(E)$, and we are interested only in the solutions where all $\xi^{\prime \prime}(E)$ are non-zero. Because of the action of $T_{\mathrm{NS}}\left(S_{r}\right)$ on the rescaling factors and as $E_{1}, \ldots, E_{r}$ are part of a basis of $\operatorname{Pic}\left(S_{r}\right)$, we may assume $\eta_{1}^{\prime \prime}=\cdots=\eta_{r}^{\prime \prime}=1$.

Consider a (1)-ruling $D=E_{1}+E$ such that $\left(E_{2}, E\right)=0$. Then

$$
D=E_{1}^{\prime}+E_{1}^{\prime \prime}=\cdots=E_{r-3}^{\prime}+E_{r-3}^{\prime \prime}=E_{1}+E=E_{2}+E^{\prime}
$$

are the $r-1$ possibilities to write $D$ as the sum of two intersecting (-1)curves. Here, $E_{i}^{\prime}, E_{i}^{\prime \prime} \in \mathcal{N}\left(E_{1}\right)_{0} \cap \mathcal{N}\left(E_{2}\right)_{0}$. As in the discussion before Lemma 4.10, we may set up the equations $F_{D, j}$ such that $g_{D, 1}$ and $g_{D, 2}$ express $\xi^{\prime \prime}(E)$ and $\xi^{\prime \prime}\left(E^{\prime}\right)$ directly as a linear combination of $\xi^{\prime \prime}\left(E_{i}^{\prime}\right) \xi^{\prime \prime}\left(E_{i}^{\prime \prime}\right)$. 
This expresses all $\xi(E) \in \Xi_{1,0}^{\prime \prime}$ and all $\xi^{\prime \prime}\left(E^{\prime}\right) \in \Xi_{0,1}^{\prime \prime}$ in terms of variables in $\Xi_{0,0}^{\prime \prime}$.

For a (1)-ruling $D=E_{1}+E$ such that $\left(E_{2}, E\right)=1$, we have

$$
D=E_{1}^{\prime}+E_{1}^{\prime \prime}=\cdots=E_{r-2}^{\prime}+E_{r-2}^{\prime \prime}=E_{1}+E,
$$

where we may assume $\left(E_{2}, E_{i}^{\prime}\right)=0$ and $\left(E_{2}, E_{i}^{\prime \prime}\right)=1$. Since $\left(E_{1}, E_{i}^{\prime}\right)=$ $\left(E_{1}, E_{i}^{\prime \prime}\right)=0$, we have $\xi^{\prime \prime}\left(E_{i}^{\prime}\right) \in \Xi_{0,0}^{\prime \prime}$ and $\xi^{\prime \prime}\left(E_{i}^{\prime \prime}\right) \in \Xi_{0,1}^{\prime \prime}$. Using the previous findings to express $\xi^{\prime \prime}\left(E_{i}^{\prime \prime}\right)$ in terms of variables $\Xi_{0,0}^{\prime \prime}$, the equation $g_{D, 1}$ results in a condition on the variables $\Xi_{0,0}^{\prime \prime}$, while $g_{D, 2}$ expresses $\xi^{\prime \prime}(E) \in \Xi_{1,1}^{\prime \prime}$ in terms of these variables.

In the case $r=7$, the equation $g_{D, 2}$ for $D=E_{1}+C_{2}$ expresses $\lambda_{2}^{\prime \prime} \in$ $\Xi_{1,2}^{\prime \prime}$ in terms of variables in $\Xi_{0,1}^{\prime \prime}$, and $g_{D, 1}$ gives a further condition on these variables. Furthermore, $g_{D, 2}$ for the (1)-ruling $D=E_{2}+C_{1}$ expresses $\lambda_{1}^{\prime \prime} \in \Xi_{2,1}^{\prime \prime}$ in terms of $\Xi_{1,0}^{\prime \prime}$, while $g_{D, 1}$ gives a further condition on them. Substituting the expressions for $\Xi_{0,1}^{\prime \prime}$ respectively $\Xi_{1,0}^{\prime \prime}$ in terms of $\Xi_{0,0}^{\prime \prime}$, we get expressions for $\lambda_{2}^{\prime \prime}$ and $\lambda_{1}^{\prime \prime}$, while the $g_{D, 1}$ result in further condition on $\Xi_{0,0}^{\prime \prime}$.

We summarize this in the following lemma. For its proof, it remains to show in the following sections that the expressions for $\Xi^{\prime \prime}\left(E_{1}\right)_{>0} \cup \Xi^{\prime \prime}\left(E_{2}\right)_{>0}$ are non-zero, and that the further conditions vanish.

Lemma 4.11. We can write the $N_{r}-N_{r-2}-2$ rescaling factors in the set $\Xi^{\prime \prime}\left(E_{1}\right)_{>0} \cup \Xi^{\prime \prime}\left(E_{2}\right)_{>0}$ as non-zero expressions in terms of $N_{r-2}+2$ rescaling factors $\Xi_{0,0}^{\prime \prime} \cup\left\{\xi^{\prime \prime}\left(E_{1}\right), \xi^{\prime \prime}\left(E_{2}\right)\right\}$. With this, the $N_{r}-2 N_{r-1}+N_{r-2}$ further conditions on the rescaling factors are trivial.

For an open subset of the $N_{r-2}+2$ parameters $\left\{\eta_{1}^{\prime \prime}, \eta_{2}^{\prime \prime}\right\} \cup \Xi_{0,0}^{\prime \prime}$, all rescaling factors are non-zero, so we obtain good rescalings, which proves Theorem 4.1 once the proof of Lemma 4.11 is completed.

\subsection{Degree 3}

In this section, we prove Lemma 4.11 for $r=6$ by solving the system of equations on the rescaling factors of Lemma 4.10. For each (1)-ruling $D \in \mathcal{M}_{6}$, we determine the coefficients of the equation $p_{D}$ defining $H_{6}$ as in (4.1), and find the coefficients $\alpha_{j}, \beta_{j}$ of $F_{D, j}$ defining $\mathbb{A}\left(S_{6}\right)$ as in (4.2) in the list in Section 3.3. This allows us to write down the 20 equations $g_{D, i}$ on the rescaling factors $\xi^{\prime \prime}(E)$ explicitly. Let

$$
\gamma_{1}:=a d-b c, \quad \gamma_{2}:=(a-1)(d-1)-(b-1)(c-1)
$$

for simplicity.

$$
\begin{aligned}
& g_{E_{1}+m_{1,2}, 1}=-\eta_{3}^{\prime \prime} \mu_{2,3}^{\prime \prime}-\eta_{4}^{\prime \prime} \mu_{2,4}^{\prime \prime}-b \eta_{5}^{\prime \prime} \mu_{2,5}^{\prime \prime}-d \eta_{6}^{\prime \prime} \mu_{2,6}^{\prime \prime}, \\
& g_{E_{1}+m_{1,2}, 2}=\eta_{1}^{\prime \prime} \mu_{1,2}^{\prime \prime}+\eta_{4}^{\prime \prime} \mu_{2,4}^{\prime \prime}+\eta_{5}^{\prime \prime} \mu_{2,5}^{\prime \prime}+\eta_{6}^{\prime \prime} \mu_{2,6}^{\prime \prime}, \\
& g_{E_{1}+m_{1,3}, 1}=-\eta_{1}^{\prime \prime} \mu_{1,3}^{\prime \prime}+\eta_{4}^{\prime \prime} \mu_{3,4}^{\prime \prime}+\eta_{5}^{\prime \prime} \mu_{3,5}^{\prime \prime}+\eta_{6}^{\prime \prime} \mu_{3,6}^{\prime \prime}, \\
& g_{E_{1}+m_{1,3}, 2}=\eta_{2}^{\prime \prime} \mu_{2,3}^{\prime \prime}+\eta_{4}^{\prime \prime} \mu_{3,4}^{\prime \prime}+a \eta_{5}^{\prime \prime} \mu_{3,5}^{\prime \prime}+c \eta_{6}^{\prime \prime} \mu_{3,6}^{\prime \prime}, \\
& g_{E_{1}+m_{1,4}, 1}=-\eta_{1}^{\prime \prime} \mu_{1,4}^{\prime \prime}+\eta_{3}^{\prime \prime} \mu_{3,4}^{\prime \prime}+(b-1) \eta_{5}^{\prime \prime} \mu_{4,5}^{\prime \prime}+(1-d) \eta_{6}^{\prime \prime} \mu_{4,6}^{\prime \prime}, \\
& g_{E_{1}+m_{1,4}, 2}=-\eta_{2}^{\prime \prime} \mu_{2,4}^{\prime \prime}+\eta_{3}^{\prime \prime} \mu_{3,4}^{\prime \prime}+(b-a) \eta_{5}^{\prime \prime} \mu_{4,5}^{\prime \prime}+(c-d) \eta_{6}^{\prime \prime} \mu_{4,6}^{\prime \prime},
\end{aligned}
$$




$$
\begin{aligned}
g_{E_{1}+m_{1,5}, 1} & =-\eta_{2}^{\prime \prime} \mu_{2,5}^{\prime \prime}+a / b \eta_{3}^{\prime \prime} \mu_{3,5}^{\prime \prime}+(a-b) / b \eta_{4}^{\prime \prime} \mu_{4,5}^{\prime \prime}+\gamma_{1} / b \eta_{6}^{\prime \prime} \mu_{5,6}^{\prime \prime}, \\
g_{E_{1}+m_{1,5}, 2} & =-\eta_{1}^{\prime \prime} \mu_{1,5}^{\prime \prime}+1 / b \eta_{3}^{\prime \prime} \mu_{3,5}^{\prime \prime}+(1-b) / b \eta_{4}^{\prime \prime} \mu_{4,5}^{\prime \prime}+(d-b) / b \eta_{6}^{\prime \prime} \mu_{5,6}^{\prime \prime}, \\
g_{E_{1}+m_{1,6}, 1} & =-\eta_{1}^{\prime \prime} \mu_{1,6}^{\prime \prime}+1 / d \eta_{3}^{\prime \prime} \mu_{3,6}^{\prime \prime}+(d-1) / d \eta_{4}^{\prime \prime} \mu_{4,6}^{\prime \prime}+(b-d) / d \eta_{5}^{\prime \prime} \mu_{5,6}^{\prime \prime}, \\
g_{E_{1}+m_{1,6}, 2} & =-\eta_{2}^{\prime \prime} \mu_{2,6}^{\prime \prime}+c / d \eta_{3}^{\prime \prime} \mu_{3,6}^{\prime \prime}+(d-c) / d \eta_{4}^{\prime \prime} \mu_{4,6}^{\prime \prime}-\gamma_{1} / d \eta_{5}^{\prime \prime} \mu_{5,6}^{\prime \prime}, \\
g_{E_{1}}+Q_{2}, 1 & =a(c-d) \eta_{1}^{\prime \prime} \lambda_{2}^{\prime \prime}+(d-1) \eta_{2}^{\prime \prime} \lambda_{1}^{\prime \prime}-\mu_{3,4}^{\prime \prime} \mu_{5,6}^{\prime \prime}+\mu_{3,6}^{\prime \prime} \mu_{4,5}^{\prime \prime}, \\
g_{E_{1}+Q_{2}, 2} & =\gamma_{1} \eta_{1}^{\prime \prime} \lambda_{2}^{\prime \prime}+(b-d) \eta_{2}^{\prime \prime} \lambda_{1}^{\prime \prime}-\mu_{3,5}^{\prime \prime} \mu_{4,6}^{\prime \prime}-\mu_{3,6}^{\prime \prime} \mu_{4,5}^{\prime \prime}, \\
g_{E_{1}+Q_{3}, 1} & =b(c-d) \eta_{1}^{\prime \prime} \lambda_{3}^{\prime \prime}+(c-1) \eta_{3}^{\prime \prime} \lambda_{1}^{\prime \prime}-\mu_{2,4}^{\prime \prime} \mu_{5,6}^{\prime \prime}+\mu_{2,6}^{\prime \prime} \mu_{4,5}^{\prime \prime}, \\
g_{E_{1}+Q_{3}, 2} & =\gamma_{1} \eta_{1}^{\prime \prime} \lambda_{3}^{\prime \prime}+(a-c) \eta_{3}^{\prime \prime} \lambda_{1}^{\prime \prime}-\mu_{2,5}^{\prime \prime} \mu_{4,6}^{\prime \prime}-\mu_{2,6}^{\prime \prime} \mu_{4,5}^{\prime \prime}, \\
g_{E_{1}+Q_{4}, 1} & =b c \eta_{1}^{\prime \prime} \lambda_{4}^{\prime \prime}+(b c-b-c+1) \eta_{4}^{\prime \prime} \lambda_{1}^{\prime \prime}-\mu_{2,3}^{\prime \prime} \mu_{5,6}^{\prime \prime}+\mu_{2,6}^{\prime \prime} \mu_{3,5}^{\prime \prime}, \\
g_{E_{1}+Q_{4}, 2} & =(a d-b c) \eta_{1}^{\prime \prime} \lambda_{4}^{\prime \prime}+\gamma_{2} \eta_{4}^{\prime \prime} \lambda_{1}^{\prime \prime}+\mu_{2,5}^{\prime \prime} \mu_{3,6}^{\prime \prime}-\mu_{2,6}^{\prime \prime} \mu_{3,5}^{\prime \prime}, \\
g_{E_{1}+Q_{5}, 1} & =(d-c) \eta_{1}^{\prime \prime} \lambda_{5}^{\prime \prime}+\gamma_{2} \eta_{5}^{\prime \prime} \lambda_{1}^{\prime \prime}-\mu_{2,4}^{\prime \prime} \mu_{3,6}^{\prime \prime}+\mu_{2,6}^{\prime \prime} \mu_{3,4}^{\prime \prime}, \\
g_{E_{1}+Q_{5}, 2} & =c \eta_{1}^{\prime \prime} \lambda_{5}^{\prime \prime}+(a-c)(1-b) \eta_{5}^{\prime \prime} \lambda_{1}^{\prime \prime}-\mu_{2,3}^{\prime \prime} \mu_{4,6}^{\prime \prime}-\mu_{2,6}^{\prime \prime} \mu_{3,4}^{\prime \prime}, \\
g_{E_{1}+Q_{6}, 1} & =(b-a) \eta_{1}^{\prime \prime} \lambda_{6}^{\prime \prime}+\gamma_{2} \eta_{6}^{\prime \prime} \lambda_{1}^{\prime \prime}+\mu_{2,4}^{\prime \prime} \mu_{3,5}^{\prime \prime}-\mu_{2,5}^{\prime \prime} \mu_{3,4}^{\prime \prime}, \\
g_{E_{1}+Q_{6}, 2} & =a \eta_{1}^{\prime \prime} \lambda_{6}^{\prime \prime}+(c-a)(d-1) \eta_{6}^{\prime \prime} \lambda_{1}^{\prime \prime}-\mu_{2,3}^{\prime \prime} \mu_{4,5}^{\prime \prime}+\mu_{2,5}^{\prime \prime} \mu_{3,4}^{\prime \prime} .
\end{aligned}
$$

As explained in the previous section, we may assume $\eta_{1}^{\prime \prime}=\cdots=\eta_{6}^{\prime \prime}=1$.

Recall the discussion before the definition 4.2 of $F_{D, j}$ and Lemma 4.10 If we had chosen $\xi_{4}=\eta_{i} \lambda_{1}$ and $\xi_{5}=\eta_{1} \lambda_{i}$ when writing down the equations $F_{E_{1}+Q_{i}, j}$ in Section 3.3 , then the resulting $g_{E_{1}+Q_{i}, 2}$ would give $\lambda_{i}^{\prime \prime}$ directly as a quadratic expression in terms of $\mu_{j, k}^{\prime \prime}$. Furthermore, each of the five $g_{E_{1}+Q_{i}, 1}$ would express $\lambda_{1}^{\prime \prime}$ as a quadratic equation in $\mu_{j, k}^{\prime \prime}$. Of course, we get the same result by solving the equivalent system of equations $g_{E_{1}+Q_{i}, j}$ as listed above.

The equations $g_{E_{1}+m_{1, i}, j}$ for $i \in\{3, \ldots, 6\}$ and $g_{E_{1}+Q_{2}, j}$ allow us to express the variables $\mu_{1, i}^{\prime \prime}, \lambda_{2}^{\prime \prime}$ in $\Xi_{1,0}^{\prime \prime}$ and $\mu_{2, i}^{\prime \prime}, \lambda_{1}^{\prime \prime}$ in $\Xi_{0,1}^{\prime \prime}$ in terms of the six variables $\mu_{3,4}^{\prime \prime}, \ldots, \mu_{5,6}^{\prime \prime} \in \Xi_{0,0}^{\prime \prime}$. (As we have set the remaining elements $\eta_{3}^{\prime \prime}, \ldots, \eta_{6}^{\prime \prime}$ of $\Xi_{0,0}^{\prime \prime}$ to the value 1 , they do not occur in these expressions.)

With $\gamma_{3}:=d(a-c)(1-b)-c(b-d)(1-a)$, we obtain:

$$
\begin{aligned}
\mu_{1,3}^{\prime \prime} & =\mu_{3,4}^{\prime \prime}+\mu_{3,5}^{\prime \prime}+\mu_{3,6}^{\prime \prime}, \\
\mu_{2,3}^{\prime \prime} & =-\mu_{3,4}^{\prime \prime}-a \mu_{3,5}^{\prime \prime}-c \mu_{3,6}^{\prime \prime}, \\
\mu_{1,4}^{\prime \prime} & =\mu_{3,4}^{\prime \prime}+(b-1) \mu_{4,5}^{\prime \prime}+(1-d) \mu_{4,6}^{\prime \prime}, \\
\mu_{2,4}^{\prime \prime} & =\mu_{3,4}^{\prime \prime}+(b-a) \mu_{4,5}^{\prime \prime}+(c-d) \mu_{4,6}^{\prime \prime}, \\
\mu_{1,5}^{\prime \prime} & =1 / b \mu_{3,5}^{\prime \prime}+(1-b) / b \mu_{4,5}^{\prime \prime}+(d-b) / b \mu_{5,6}^{\prime \prime}, \\
\mu_{2,5}^{\prime \prime} & =a / b \mu_{3,5}^{\prime \prime}+(a-b) / b \mu_{4,5}^{\prime \prime}+\gamma_{1} / b \mu_{5,6}^{\prime \prime}, \\
\mu_{1,6}^{\prime \prime} & =1 / d \mu_{3,6}^{\prime \prime}+(d-1) / d \mu_{4,6}^{\prime \prime}+(b-d) / d \mu_{5,6}^{\prime \prime}, \\
\mu_{2,6}^{\prime \prime} & =c / d \mu_{3,6}^{\prime \prime}+(d-c) / d \mu_{4,6}^{\prime \prime}-\gamma_{1} / d \mu_{5,6}^{\prime \prime}, \\
\lambda_{1}^{\prime \prime} & =-\gamma_{1} / \gamma_{3} \mu_{3,4}^{\prime \prime} \mu_{5,6}^{\prime \prime}-a(d-c) / \gamma_{3} \mu_{3,5}^{\prime \prime} \mu_{4,6}^{\prime \prime}-c(b-a) / \gamma_{3} \mu_{3,6}^{\prime \prime} \mu_{4,5}^{\prime \prime}, \\
\lambda_{2}^{\prime \prime} & =(b-d) / \gamma_{3} \mu_{3,4}^{\prime \prime} \mu_{5,6}^{\prime \prime}+(1-d) / \gamma_{3} \mu_{3,5}^{\prime \prime} \mu_{4,6}^{\prime \prime}+(1-b) / \gamma_{3} \mu_{3,6}^{\prime \prime} \mu_{4,5}^{\prime \prime} .
\end{aligned}
$$

We consider the remaining equations $g_{E_{1}+E, i}$ for $E \in\left\{m_{1,2}, Q_{3}, \ldots, Q_{6}\right\}$. We can use $g_{E_{1}+E, 2}$ and substitution of our previous results in order to 
express $\xi^{\prime \prime}(E) \in \Xi_{1,1}^{\prime \prime}$ in terms of $\Xi_{0,0}^{\prime \prime}$ :

$$
\begin{aligned}
\mu_{1,2}^{\prime \prime}= & \mu_{3,4}^{\prime \prime}-a / b \mu_{3,5}^{\prime \prime}-c / d \mu_{3,6}^{\prime \prime}+(a-b)(b-1) / b \mu_{4,5}^{\prime \prime} \\
& +(d-c)(d-1) / d \mu_{4,6}^{\prime \prime}+(b-d) \gamma_{1} /(b d) \mu_{5,6}^{\prime \prime}, \\
\lambda_{3}^{\prime \prime}= & (a-c) / \gamma_{3} \mu_{3,4}^{\prime \prime} \mu_{5,6}^{\prime \prime}+a(1-c) /\left(b \gamma_{3}\right) \mu_{3,5}^{\prime \prime} \mu_{4,6}^{\prime \prime}+c(1-a) /\left(d \gamma_{3}\right) \mu_{3,6}^{\prime \prime} \mu_{4,5}^{\prime \prime} \\
& +1 /(b d) \mu_{4,5}^{\prime \prime} \mu_{4,6}^{\prime \prime}-1 / d \mu_{4,5}^{\prime \prime} \mu_{5,6}^{\prime \prime}+1 / b \mu_{4,6}^{\prime \prime} \mu_{5,6}^{\prime \prime}, \\
\lambda_{4}^{\prime \prime}= & \gamma_{2} / \gamma_{3} \mu_{3,4}^{\prime \prime} \mu_{5,6}^{\prime \prime}-1 /(b d) \mu_{3,5}^{\prime \prime} \mu_{3,6}^{\prime \prime}+(1-d)(c-d)(a-1) /\left(d \gamma_{3}\right) \mu_{3,5}^{\prime \prime} \mu_{4,6}^{\prime \prime} \\
& -1 / d \mu_{3,5}^{\prime \prime} \mu_{5,6}^{\prime \prime}+(1-c)(b-1)(a-b) /\left(b \gamma_{3}\right) \mu_{3,6}^{\prime \prime} \mu_{4,5}^{\prime \prime}-1 / b \mu_{3,6}^{\prime \prime} \mu_{5,6}^{\prime \prime}, \\
\lambda_{5}^{\prime \prime}= & 1 / d \mu_{3,4}^{\prime \prime} \mu_{3,6}^{\prime \prime}-1 / d \mu_{3,4}^{\prime \prime} \mu_{4,6}^{\prime \prime}+(b-d)(1-a) \gamma_{1} /\left(d \gamma_{3}\right) \mu_{3,4}^{\prime \prime} \mu_{5,6}^{\prime \prime} \\
& +a \gamma_{2} / \gamma_{3} \mu_{3,5}^{\prime \prime} \mu_{4,6}^{\prime \prime}+(b-1)(a-b)(a-c) / \gamma_{3} \mu_{3,6}^{\prime \prime} \mu_{4,5}^{\prime \prime}-\mu_{3,6}^{\prime \prime} \mu_{4,6}^{\prime \prime}, \\
\lambda_{6}^{\prime \prime}= & -1 / b \mu_{3,4}^{\prime \prime} \mu_{3,5}^{\prime \prime}-1 / b \mu_{3,4}^{\prime \prime} \mu_{4,5}^{\prime \prime}+(1-c)(b-d) \gamma_{1} /\left(b \gamma_{3}\right) \mu_{3,4}^{\prime \prime} \mu_{5,6}^{\prime \prime} \\
& -\mu_{3,5}^{\prime \prime} \mu_{4,5}^{\prime \prime}+(d-1)(c-d)(a-c) / \gamma_{3} \mu_{3,5}^{\prime \prime} \mu_{4,6}^{\prime \prime}+c \gamma_{2} / \gamma_{3} \mu_{3,6}^{\prime \prime} \mu_{4,5}^{\prime \prime}
\end{aligned}
$$

Finally, we check by substituting and expanding that the five further conditions $g_{E_{1}+E, 1}$ are trivial.

Using the restrictions on $a, b, c, d$ imposed by the fact that $p_{1}, \ldots, p_{6}$ are in general position (e.g., $a$ must be different from $b$ and $c$, and all are neither 0 nor 1$)$, we see that $\mu_{1,2}^{\prime \prime}, \ldots, \mu_{2,6}^{\prime \prime}, \lambda_{1}^{\prime \prime}, \ldots, \lambda_{6}^{\prime \prime}$ are non-zero polynomials in $\mu_{3,4}^{\prime \prime}, \ldots, \mu_{5,6}^{\prime \prime}$. Therefore, for an open subset of the $N_{4}+2=12$ parameters $\eta_{1}^{\prime \prime}, \ldots, \eta_{6}^{\prime \prime}, \mu_{3,4}^{\prime \prime}, \ldots, \mu_{5,6}^{\prime \prime}$, all rescaling factors are non-zero, resulting in good rescalings.

\subsection{Degree 2}

For the proof of Lemma 4.11 for $r=7$, we proceed as in the case $r=6$ and assume $\eta_{1}^{\prime \prime}=\cdots=\eta_{7}^{\prime \prime}=1$.

Let $D:=D_{i}^{(1)} \in \mathcal{M}_{7}$ for $i \in\{3, \ldots, 7\}$. We can arrange $F_{D, 1}, \ldots F_{D, 4}$ in such a way that $g_{D, 1}$ and $g_{D, 2}$ express $\mu_{1, i}^{\prime \prime}$ and $\mu_{2, i}^{\prime \prime}$ in terms of $\mu_{i, j}^{\prime \prime}$ for $j \in\{3, \ldots, 7\} \backslash\{i\}$ (see the discussion before Lemma 4.10). Similarly, for $i \in\{3, \ldots, 7\}$ and $D:=D_{1,2, i}^{(2)} \in \mathcal{M}_{7}$, we can arrange $g_{D, 1}$ and $g_{D, 2}$ such that they express $\nu_{1, i}^{\prime \prime}$ and $\nu_{2, i}^{\prime \prime}$ linearly in $\nu_{1,2}^{\prime \prime}$ and of degree 2 in $\mu_{3,4}^{\prime \prime}, \ldots, \mu_{6,7}^{\prime \prime}$. This expresses all variables in $\Xi_{0,1}^{\prime \prime} \cup \Xi_{1,0}^{\prime \prime}$ in terms of $\Xi_{0,0}^{\prime \prime}$.

Substituting this into an appropriately arranged $g_{D, 2}$ for $D:=D_{2}^{(1)} \in$ $\mathcal{M}_{7}$ gives $\mu_{1,2}^{\prime \prime} \in \Xi_{1,1}^{\prime \prime}$ in terms of $\mu_{3,4}^{\prime \prime}, \ldots, \mu_{6,7}^{\prime \prime}$, and we check that $g_{D, 1}$ becomes trivial.

Now, let $D:=D_{1, i, j}^{(2)} \in \mathcal{M}_{7}$ for $i<j \in\{3, \ldots, 7\}$. We arrange $g_{D, 1}$ and $g_{D, 2}$ such that they express $\nu_{1, i}^{\prime \prime}$ respectively $\nu_{i, j}^{\prime \prime}$ in terms of $\nu_{1, j}^{\prime \prime}$ and expressions of degree 2 in $\mu_{k, l}^{\prime \prime}$. Using our previous findings, the first expression turns out trivial, and the second one gives $\nu_{i, j}^{\prime \prime} \in \Xi_{1,1}^{\prime \prime}$ in terms of $\nu_{1,2}^{\prime \prime}, \mu_{3,4}^{\prime \prime}, \ldots, \mu_{6,7}^{\prime \prime} \in \Xi_{0,0}^{\prime \prime}$.

Finally, let $D \in\left\{D_{1,2}^{(3)}, \ldots, D_{1,7}^{(3)}, D_{2,1}^{(3)}\right\} \subset \mathcal{M}_{7}$. We arrange $g_{D, 1}$ and $g_{D, 2}$ such that the first one is an expression in $\mu_{j, k}^{\prime \prime}$ and $\nu_{j, k}^{\prime \prime}$ which becomes trivial. The second one expresses $\lambda_{i}^{\prime \prime}$ in terms of $\mu_{j, k}^{\prime \prime}$ and $\nu_{j, k}^{\prime \prime}$, and we substitute again our previous results. 
This completes the proof of Lemma 4.11 and thus Theorem 4.1. In total, we obtain good rescalings for an open subset of a system $N_{5}+2=18$ parameters

$$
\eta_{1}^{\prime \prime}, \ldots, \eta_{7}^{\prime \prime}, \quad \mu_{3,4}^{\prime \prime}, \ldots, \mu_{6,7}^{\prime \prime}, \quad \nu_{1,2}^{\prime \prime} .
$$

Since it is straightforward to determine the exact expressions for the remaining 38 rescaling factors in terms of these parameters, and since the expressions are rather long, we choose not to list them here.

REMARK 4.12. In principle, it would be possible to consider the conditions $g_{D, i}$ for all (1)-rulings without reducing to the subset $\mathcal{M}_{r}$ as we did in Section 4.2. While this is doable in degree 3 with some software help (Magma), especially the expressions corresponding to the (1)-ruling $D_{i}^{(5)}$ in degree 2 seem to be out of reach for direct computations. Furthermore, we would have to embed the relations $v_{i}^{i}$ corresponding to the (2)-ruling $-K_{S_{7}}$, which causes further complications.

REMARK 4.13. For $r \in\{5,6,7\}$, there is a $\left(N_{r-2}-r+1\right)$-parameter family of images of $\mathbb{A}\left(S_{r}\right)$ under good embeddings in $H_{r}$ by Remark 4.2 . The dimension of $\mathbb{A}\left(S_{r}\right)$ is $r+3$, and there is a $(2 \cdot(r-4))$-parameter family of smooth Del Pezzo surfaces $S_{r}$ of degree $9-r$. The dimension of $H_{r}$ is $N_{r-1}+1$.

In fact, [Pop01, Section 6.3] shows that the closure of the union of all these images for all Del Pezzo surfaces of degree 4 equals $H_{5}$.

For $r=6$, by comparing the dimensions and numbers of parameters, a similar result seems possible. However, for $r=7$, we have

$$
\left(N_{r-2}-r+1\right)+(r+3)+2 \cdot(r-4)=26,
$$

while $H_{7}$ has dimension $N_{r-1}+1=28$. Consequently, the closure of the union of the corresponding images, over all Del Pezzo surfaces of degree 2, under all good embeddings cannot be $H_{7}$ for dimension reasons. 


\section{CHAPTER 5}

\section{Universal torsors which are hypersurfaces}

\subsection{Introduction}

The universal torsor of a generalized Del Pezzo surface $S$ has one of the following three types (Lemma 2.13):

- The universal torsor can be presentable as a Zariski open subset of affine space. By Lemma 2.12 and Proposition 2.10, this is true exactly when $S$ is toric.

- The universal torsor can be a Zariski open subset of a hypersurface in affine space.

- In other cases, the universal torsor is more complicated: It can be an open subset of an affine variety that has codimension $\geq 2$ in affine space.

We focus on universal torsors of the second type. A first example, the universal torsor over a cubic surface of type $\mathbf{E}_{6}$, has been worked out by Hassett and Tschinkel [HT04].

Universal torsors are closely connected to the Cox rings of Del Pezzo surfaces (Chapter 2). By Lemma 2.13, the question which of the three cases above a universal torsor belongs to is equivalent to the question whether the Cox ring with a minimal set of homogeneous generators (cf. Lemma 2.5) is a polynomial ring without relations, with one relation up to scalar multiples, or with more relations.

In this chapter, we determine for all types of generalized Del Pezzo surfaces of degree $\geq 3$ what kind of Cox ring they have (cf. Table 5.1). Our results were first made available as $[$ Der06c].

\begin{tabular}{|c||c|c|c|}
\hline degree & toric & one relation & more than one relation \\
\hline \hline 9 & $\mathbb{P}^{2}$ & - & - \\
8 & 2 smooth, 1 singular & - & - \\
7 & 1 smooth, 1 singular & - & - \\
6 & 1 smooth, 3 singular & 2 singular & - \\
5 & 2 singular & 4 singular & 1 smooth \\
4 & 3 singular & 7 singular & 1 smooth, 5 singular \\
3 & 1 singular & 7 singular & 1 smooth, 12 singular \\
\hline
\end{tabular}

Theorem 5.1. The Cox rings of generalized Del Pezzo surfaces whose degree is at least 3 have the following properties:

- In degree at least 7, all generalized Del Pezzo surfaces are toric. 
- In degree 6, two types $\mathbf{A}_{1}$ (with three lines), $\mathbf{A}_{2}$ have a Cox ring with 7 generators and one relation. The smooth and three types $\mathbf{A}_{1}$ (with four lines), $2 \mathbf{A}_{1}, \mathbf{A}_{2}+\mathbf{A}_{1}$ are toric.

- In degree 5, four types $\mathbf{A}_{1}, \mathbf{A}_{2}, \mathbf{A}_{3}, \mathbf{A}_{4}$ have a Cox ring with 8 generators and one relation. The smooth surface has a Cox ring with more generators and relations, and two types $2 \mathbf{A}_{1}, \mathbf{A}_{2}+\mathbf{A}_{1}$ are toric.

- In degree 4, seven types $3 \mathbf{A}_{1}, \mathbf{A}_{2}+\mathbf{A}_{1}, \mathbf{A}_{3}$ (with five lines), $\mathbf{A}_{3}+\mathbf{A}_{1}$, $\mathbf{A}_{4}, \mathbf{D}_{4}, \mathbf{D}_{5}$ have a Cox ring with 9 generators and one relation. The smooth surface and five types $\mathbf{A}_{1}, 2 \mathbf{A}_{1}$ (with eight or nine lines), $\mathbf{A}_{2}, \mathbf{A}_{3}$ (with four lines) have a Cox ring with more generators and relations, and three types $4 \mathbf{A}_{1}, \mathbf{A}_{2}+2 \mathbf{A}_{1}, \mathbf{A}_{3}+2 \mathbf{A}_{1}$ are toric.

- In degree 3, seven types $\mathbf{D}_{4}, \mathbf{A}_{3}+2 \mathbf{A}_{1}, 2 \mathbf{A}_{2}+\mathbf{A}_{1}, \mathbf{A}_{4}+\mathbf{A}_{1}, \mathbf{D}_{5}$, $\mathbf{A}_{5}+\mathbf{A}_{1}, \mathbf{E}_{6}$ have a Cox ring with 10 generators and one relation. The smooth surface and 12 types $\mathbf{A}_{1}, 2 \mathbf{A}_{1}, \mathbf{A}_{2}, 3 \mathbf{A}_{1}, \mathbf{A}_{2}+\mathbf{A}_{1}, \mathbf{A}_{3}$, $4 \mathbf{A}_{1}, \mathbf{A}_{2}+2 \mathbf{A}_{1}, \mathbf{A}_{3}+\mathbf{A}_{1}, 2 \mathbf{A}_{2}, \mathbf{A}_{4}, \mathbf{A}_{5}$ have a Cox ring with more generators and relations, and the type $3 \mathbf{A}_{2}$ is toric.

In Section 5.2, we describe the steps which must be taken to calculate the Cox ring of each generalized Del Pezzo surface $S$ explicitly in case there is exactly one relation in $\operatorname{Cox}(S)$.

In Sections 5.3, 5.4, 5.5, and 5.6, we check for every type of degree $\geq 3$ which of the three cases it belongs to. For each type with exactly one relation, we calculate the Cox ring, and we list the data which is most important for its calculation and applications: We give a "nice" model which is defined over $\mathbb{Q}$, its singularities, its lines (which are defined over $\mathbb{Q}$ ), generators of the Picard group, the effective cone, generators of the Cox ring, the extended Dynkin diagram, and the map from the universal torsor to the Del Pezzo surface.

\subsection{Strategy of the proofs}

In this section, we give an overview of the method of Hassett and Tschinkel [HT04] to calculate the Cox ring of generalized Del Pezzo surfaces in cases where the Cox ring has exactly one relation.

By Lemma 2.7, the Cox ring has one generating section for every negative curve. In some cases, the Cox ring is generated by these sections, but this is not always true. In that case, we look for extra generators in nef degrees (Lemma 2.8). When we find enough generators, we have a relation which can be calculated explicitly once we know details about the anticanonical embedding.

In more detail, we perform the following steps: Let $S$ be a smooth or singular Del Pezzo surface of degree $9-r \geq 3$, given by the vanishing of some homogeneous polynomials for its anticanonical embedding into $\mathbb{P}^{9-r}$. Let $\widetilde{S}$ be its minimal desingularization. We apply this method only in the cases where the Cox ring will turn out to have exactly $r+4$ generators and one relation. In the other cases, it would fail.

Find the extended Dynkin diagram of the negative curves. For this, first we search for the lines on $S$, by looking at the equations of the 
anticanonical embedding of $S$ (Remark 1.26). Using the classifications of BW79 for degree 3 and [CT88 for degrees $\geq 4$, we know when we are done. Then we determine the singularities, resolve them by blow-ups, and keep track of the intersection behavior of the exceptional divisors with the transforms of the lines. This must be done explicitly for degree 3 , and the results can be found in $\mathbf{C T 8 8}$ for degrees $\geq 4$. The information is summarized in an extended Dynkin diagram of negative curves. Note that the diagrams can also be constructed from the information given in AN04, Table 3], as explained in Section 1.4.

Determine a basis of the Picard group. We need a $\mathbb{Z}$-basis for $\operatorname{Pic}(\widetilde{S}) \cong \mathbb{Z}^{r+1}$ : We test for different $(r+1)$-element subsets of the negative curves whether their intersection matrix has determinant \pm 1 . Once we have found such a subset, we call its elements $E_{1}, \ldots, E_{r+1}$, and the remaining negative curves are called $E_{r+2}, \ldots, E_{t}$. By considering their intersection with the basis, we determine $E_{r+2}, \ldots, E_{t}$ in terms of this basis. Using the adjunction formula, we calculate the anticanonical class $-K_{\widetilde{S}}$, which is nef since it describes the anticanonical embedding of $S$.

Determine the effective cone and its dual, the nef cone. In every case, we want to show that the effective cone is generated by the negative curves $E_{1}, \ldots, E_{t}$. Arguing as in [HT04, Proposition 3.5], we only need to check that the cone generated by $E_{1}, \ldots, E_{t}$ contains its dual, which is generated by some divisors $A_{1}, \ldots, A_{u}$. This can be done by an explicit calculation using the basis we found in the previous step. It turns out to be true in every case. We will show this result in general in Proposition 8.11. Therefore, $A_{1}, \ldots, A_{u}$ generate the nef cone. By Lemma 2.7, a generating set of $\operatorname{Cox}(\widetilde{S})$ must contain a non-zero section $\eta_{i} \in H^{0}\left(E_{i}\right)$ for every $i \in$ $\{1, \ldots, t\}$.

Find generating sections in degrees of generators of the nef cone. To every $A_{i}$, we can associate a map from $\widetilde{S}$ to projective space. By looking at these maps carefully, we can find extra generators $\alpha_{i} \in H^{0}\left(A_{i}\right)$ of the Cox ring. We obtain generators $\eta_{1}, \ldots, \eta_{t}, \alpha_{1}, \ldots, \alpha_{s}$, where $t+s=r+4$, which fulfill a certain relation. Assuming that this is a generating set with one relation, we check that this gives exactly the right number of independent sections in each $H^{0}\left(A_{i}\right)$, where $\operatorname{dim} H^{0}\left(A_{i}\right)=\chi\left(A_{i}\right)$ can be calculated using Riemann-Roch and Kawamata-Viehweg as in [HT04, Corollary 1.10].

Determine $\phi^{*}\left(x_{i}\right)$ for the anticanonical embedding. Especially by considering projections from the singularities and lines on the one hand, and maps to $\mathbb{P}^{2}$ corresponding to the fact that $S$ is a blow-up of $\mathbb{P}^{2}$ in $r$ points on the other hand, and by considering the corresponding transformations of the extended Dynkin diagrams, we find out which projections

$$
\psi_{i}: \mathbf{x} \mapsto\left(x_{j_{1}}: \cdots: x_{j_{n}}\right)
$$

are given by $A_{i}$, and what $\psi_{i}^{*}\left(x_{j_{l}}\right) \in H^{0}\left(A_{i}\right)$ is. Finally, we combine this information in order to get $\phi^{*}\left(x_{i}\right) \in H^{0}\left(-K_{\widetilde{S}}\right)$ for

$$
\phi: \widetilde{S} \rightarrow S \hookrightarrow \mathbb{P}^{9-r} .
$$

Using the equations defining $S$, we derive a relation $R$ from this. Note that even though $S$ might be defined by more than one equation, this gives only 
one relation in the cases we consider. We obtain a map

$$
\varrho: \mathbb{K}\left[\eta_{1}, \ldots, \eta_{t}, \alpha_{1}, \ldots, \alpha_{s}\right] /(R) \rightarrow \operatorname{Cox}(\widetilde{S}) .
$$

Prove injectivity of $\varrho$. The argument is the same as in [HT04, Theorem 3.8]. We must check that $-K_{\widetilde{S}}$ is in the inner of the effective cone.

Prove surjectivity of $\varrho$. We follow the proof of [HT04, Proposition 3.9]. We need to check that the generators of the effective cone $A_{1}, \ldots, A_{u}$ are contained in the moving cone corresponding to the $r+4$ divisors $E_{1}, \ldots, E_{t}, A_{1}, \ldots, A_{s}$ (cf. [HT04, Lemma 3.11]). Furthermore, we check that

$$
\sum_{i=1}^{t} E_{i}+\sum_{j=1}^{s} A_{i}=\operatorname{deg}(R)+A
$$

for some nef divisor $A$ (cf. [HT04, Proposition 3.12]).

Remark 5.2. For many Del Pezzo surfaces $S$, the number of negative curves on its minimal desingularization $\widetilde{S}$ is at least $r+5$, so Lemma 2.7 and Lemma 2.13 imply that $\operatorname{Cox}(\widetilde{S})$ has at least two relations.

On the other hand, in many cases where the number of negative curves on $\widetilde{S}$ is at most $r+4$, the surfaces turn out to be toric or to have exactly one relation in $\operatorname{Cox}(\widetilde{S})$.

However, $\widetilde{S}$ may have $r+4$ or fewer negative curves, but two or more relations (see Proposition 5.6 for type viii of degree 4, and Proposition 5.7 for types $x i$ and $x v i$ of degree 3). In these cases, more work must be done to see that the number of relations is actually at least two.

In the next sections, we go through all types of Del Pezzo surfaces of degrees $\geq 3$ whose Cox rings have exactly one relation, and list the following information for one example of each type:

- Equations for the anticanonical embedding are given. We choose "nice" equations in the sense that they are defined over $\mathbb{Z}$, all the coefficients are \pm 1 , and the singularities and lines have "nice" forms. For applications, it can be useful to know these equations.

- The equations of the singularities and lines on the anticanonical embedding are given, as we need their names $E_{i}$ later. Furthermore, they are needed in applications.

- The ordering of the $E_{i}$ is chosen in such a way that $E_{1}, \ldots, E_{r+1}$ are a $\mathbb{Z}$-basis of $\operatorname{Pic}(\widetilde{S})$. We list the other $E_{i}$ and $-K_{\widetilde{S}}$ in terms of this basis, as this is needed for further calculations.

- We list generators and the relation of $\operatorname{Cox}(\widetilde{S})$. Here, $\eta_{i}$ is always a non-zero section of $H^{0}\left(E_{i}\right)$, which is unique up to a scalar factor. For each extra generator $\alpha_{i}$, we give the degree in terms of our basis of $\operatorname{Pic}(\widetilde{S})$, specify which projection $\psi_{i}: x \mapsto\left(x_{j_{1}}: \cdots: x_{j_{n}}\right)$ it defines, and what $\alpha_{i}=\psi_{i}^{*}\left(x_{j_{l}}\right)$ and the corresponding divisor $A_{i}$ is. We also describe the image of $A_{i}$ under the projection $\psi: \widetilde{S} \rightarrow S$. We give the equation and the degree of the relation.

- We give the extended Dynkin diagram of the negative curves as in Example 1.27, and we also include the divisors $A_{1}, \ldots, A_{s}$ (where an edge means in this chapter that the intersection number is $\geq 1$ ). 
This diagram is probably the most important piece of information, as it is independent of the actual equations of the surface, and most other information is obtained from this diagram in some way.

- For the anticanonical embedding $\phi$, we list all $\phi^{*}\left(x_{i}\right) \in H^{0}\left(-K_{\widetilde{S}}\right)$. Here, we use the notation

$$
\eta^{\left(a_{1}, \ldots, a_{r+1}\right)}:=\eta_{1}^{a_{1}} \cdots \eta_{r+1}^{a_{r+1}} .
$$

\subsection{Degree $\geq 6$}

Proposition 5.3. All generalized Del Pezzo surfaces of degree $\geq 7$ are toric.

Proof. These types are listed in [CT88, Proposition 8.1]. By Table1.4. they are toric.

\begin{tabular}{|c||c|c|c|}
\hline type & singularities & number of lines & type \\
\hline \hline 0 & - & 6 & toric \\
$i$ & $\mathbf{A}_{1}$ & 4 & toric \\
$i i$ & $\mathbf{A}_{1}$ & 3 & 1 relation \\
$i i i$ & $2 \mathbf{A}_{1}$ & 2 & toric \\
$i v$ & $\mathbf{A}_{2}$ & 2 & 1 relation \\
$v$ & $\mathbf{A}_{2}+\mathbf{A}_{1}$ & 1 & toric \\
\hline
\end{tabular}

TABle 5.2. Del Pezzo surfaces of degree 6

Proposition 5.4. Let $S$ be a generalized Del Pezzo surface of degree 6, which has one of the types of Table 5.2.

- Types $0, i, i i i, v$ are toric.

- Types ii and iv have Cox rings with seven generators and one relation.

Proof. The classification of singular Del Pezzo surfaces of degree 6 can be found in [CT88, Proposition 8.3]. Table 1.4 lists the toric types. The Cox rings of the remaining types are calculated in the rest of this section.

Type ii $\left(\mathbf{A}_{1}\right)$. It has the following properties:

- It is the intersection of nine quadrics in $\mathbb{P}^{6}$ :

$$
\begin{aligned}
& x_{0}^{2}-x_{1} x_{3}=x_{4} x_{0}-x_{2} x_{3}=x_{2} x_{0}-x_{1} x_{4}=x_{5} x_{0}-x_{2} x_{4} \\
= & x_{5} x_{1}-x_{2}^{2}=x_{5} x_{3}-x_{4}^{2}=x_{0}^{2}+x_{0} x_{3}+x_{6} x_{4} \\
= & x_{0} x_{1}+x_{0}^{2}+x_{6} x_{2}=x_{0} x_{2}+x_{0} x_{4}+x_{6} x_{5} .
\end{aligned}
$$

- Its singularity $(0: 0: 0: 0: 0: 0: 1)$ gives the exceptional divisor $E_{1}$, and its lines are $E_{2}=\left\{x_{0}=x_{1}=x_{2}=x_{4}=x_{5}=0\right\}$, $E_{3}=\left\{x_{0}=x_{2}=x_{3}=x_{4}=x_{5}=0\right\}$, and $E_{4}=\left\{x_{2}=x_{4}=x_{5}=\right.$ $\left.x_{0}+x_{1}=x_{0}+x_{3}=0\right\}$.

- A basis of $\operatorname{Pic}(\widetilde{S})$ is given by $E_{1}, \ldots, E_{4}$, with $-K_{\widetilde{S}}=(3,2,2,2)$. 
- The Cox ring is

$$
\operatorname{Cox}(\widetilde{S})=\mathbb{K}\left[\eta_{1}, \ldots, \eta_{4}, \alpha_{1}, \alpha_{2}, \alpha_{3}\right] /\left(\eta_{2} \alpha_{1}+\eta_{3} \alpha_{2}+\eta_{4} \alpha_{3}\right),
$$

where the degree of the relation is $(1,1,1,1)$, and $\operatorname{deg}\left(\alpha_{1}\right)=A_{1}=$ $(1,0,1,1)=\psi^{*}\left(\left\{\left(0: 0: 0: a^{2}: a b: b^{2}: 0\right)\right\}\right), \operatorname{deg}\left(\alpha_{2}\right)=A_{2}=$ $(1,1,0,1)=\psi^{*}\left(\left\{\left(0: a^{2}: a b: 0: 0: b^{2}: 0\right) \mid x_{1} x_{5}-x_{2}^{2}=0\right\}\right)$, and $\operatorname{deg}\left(\alpha_{3}\right)=A_{3}=(1,1,1,0)=\psi^{*}\left(\left\{\left(a^{2}:-a^{2}: a b:-a^{2}:-a b:-b^{2}:\right.\right.\right.$ $0)\}$ ), where $\psi$ is the projection $\widetilde{S} \rightarrow S$.

- The extended Dynkin diagram is:

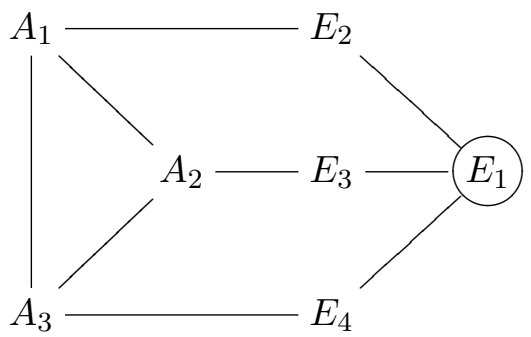

Here, the divisors $A_{1}, A_{2}, A_{3}$ meet in $(0: 0: 0: 0: 0: 1: 0)$.

- The anticanonical embedding is given by

$$
\begin{gathered}
\left(\phi^{*}\left(x_{i}\right)\right)=\left(\eta^{(1,1,1,0)} \alpha_{1} \alpha_{2}, \eta^{(1,2,0,0)} \alpha_{1}^{2}, \eta^{(2,2,1,1)} \alpha_{1}, \eta^{(1,0,2,0)} \alpha_{2}^{2},\right. \\
\left.\eta^{(2,1,2,1)} \alpha_{2}, \eta^{(3,2,2,2)}, \alpha_{1} \alpha_{2} \alpha_{3}\right),
\end{gathered}
$$

and furthermore, $\phi^{*}\left(-x_{0}-x_{1}\right)=\eta^{(1,1,0,1)} \alpha_{1} \alpha_{3}, \phi^{*}\left(-x_{0}-x_{3}\right)=$ $\eta^{(1,0,1,1)} \alpha_{2} \alpha_{3}, \phi^{*}\left(-x_{2}-x_{4}\right)=\eta^{(2,1,1,2)} \alpha_{3}$, and $\phi^{*}\left(2 x_{0}+x_{1}+x_{3}\right)=$ $\eta^{(1,0,0,2)} \alpha_{3}^{2}$.

Type iv $\left(\mathbf{A}_{2}\right)$. This surface has the following properties:

- It is the intersection of the following nine quadrics in $\mathbb{P}^{6}$ :

$$
\begin{aligned}
& x_{0} x_{5}-x_{3} x_{4}=x_{0} x_{6}-x_{1} x_{4}=x_{0} x_{6}-x_{2} x_{3}=x_{3} x_{6}-x_{1} x_{5} \\
= & x_{4} x_{6}-x_{2} x_{5}=x_{1} x_{6}+x_{3}^{2}+x_{3} x_{4}=x_{2} x_{6}+x_{3} x_{4}+x_{4}^{2} \\
= & x_{6}^{2}+x_{3} x_{5}+x_{4} x_{5}=x_{1} x_{2}+x_{0} x_{3}+x_{0} x_{4}=0 .
\end{aligned}
$$

- Its singularity $(1: 0: 0: 0: 0: 0: 0)$ gives the exceptional divisors $E_{1}, E_{2}$, and its lines are $E_{3}=\left\{x_{2}=x_{3}=x_{4}=x_{5}=x_{6}=0\right\}$ and $E_{4}=\left\{x_{1}=x_{3}=x_{4}=x_{5}=x_{6}=0\right\}$.

- A basis of $\operatorname{Pic}(\widetilde{S})$ is given by $E_{1}, \ldots, E_{4}$, with $-K_{\widetilde{S}}=(4,2,3,3)$.

- The Cox ring is

$\operatorname{Cox}(\widetilde{S})=\mathbb{K}\left[\eta_{1}, \ldots, \eta_{4}, \alpha_{1}, \alpha_{2}, \alpha_{3}\right] /\left(\eta_{2} \alpha_{1}^{2}+\eta_{3} \alpha_{2}+\eta_{4} \alpha_{3}\right)$,

where the relation is of degree $(2,1,2,2)$, and $\operatorname{deg}\left(\alpha_{1}\right)=A_{1}=$ $(1,0,1,1)=\psi^{*}\left(\left\{\left(a^{2}: 0: 0: a b:-a b: b^{2}: 0\right)\right\}\right)$ gives the projection $\psi_{1}: x \mapsto\left(x_{5}: x_{6}\right)$ with $\alpha_{1}=\phi^{*}\left(x_{6}\right), \operatorname{deg}\left(\alpha_{2}\right)=A_{2}=(2,1,1,2)=$ $\psi^{*}\left(\left\{\left(0: 0: a^{3}:-a^{2} b: b^{3}:-a b^{2}\right)\right\}\right)$ gives the projection $\psi_{2}: x \mapsto$ $\left(x_{3}: x_{5}: x_{6}\right)$ with $\alpha_{2}=\psi_{2}^{*}\left(x_{3}\right)$, and $\operatorname{deg}\left(\alpha_{3}\right)=A_{3}=(2,1,2,1)=$ $\psi^{*}\left(\left\{\left(0: a^{3}: 0:-a^{2} b: 0: b^{3}:-a b^{2}\right)\right\}\right)$ gives the projection $\psi_{3}: x \mapsto\left(x_{4}: x_{5}: x_{6}\right)$ with $\alpha_{3}=\psi_{3}^{*}\left(x_{4}\right)$. 
- The extended Dynkin diagram is:

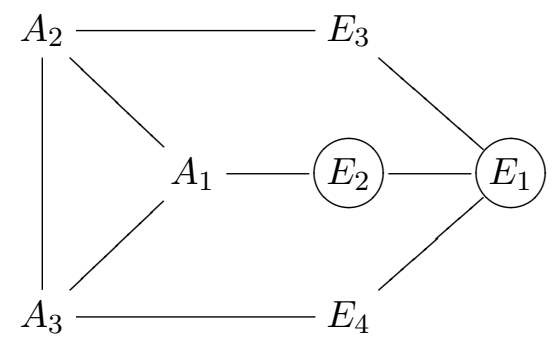

Here, $A_{1}, A_{2}, A_{3}$ meet in $(0: 0: 0: 0: 0: 1: 0)$.

- The anticanonical embedding is given by

$$
\begin{aligned}
\left(\phi^{*}\left(x_{i}\right)\right)= & \left(\alpha_{2} \alpha_{3}, \eta^{(1,1,1,0)} \alpha_{1} \alpha_{2}, \eta^{(1,1,0,1)} \alpha_{1} \alpha_{3},\right. \\
& \left.\eta^{(2,1,2,1)} \alpha_{2}, \eta^{(2,1,1,2)} \alpha_{3}, \eta^{(4,2,3,3)}, \eta^{(3,2,2,2)} \alpha_{1}\right),
\end{aligned}
$$

and furthermore, $\phi^{*}\left(-x_{1}-x_{2}\right)=\eta^{(1,2,0,0)} \alpha_{1}^{3}$ and $\phi^{*}\left(-x_{3}-x_{4}\right)=$ $\eta^{(2,2,1,1)} \alpha_{1}^{2}$.

\subsection{Degree 5}

By [CT88, Proposition 8.4], Table 5.3 lists all types of Del Pezzo surfaces of degree 5 .

\begin{tabular}{|c||c|c|c|}
\hline type & singularities & number of lines & type \\
\hline \hline 0 & - & 10 & $\geq 2$ relations \\
$i$ & $\mathbf{A}_{1}$ & 7 & 1 relation \\
$i i$ & $2 \mathbf{A}_{1}$ & 5 & toric \\
$i i i$ & $\mathbf{A}_{2}$ & 4 & 1 relation \\
$i v$ & $\mathbf{A}_{2}+\mathbf{A}_{1}$ & 3 & toric \\
$v$ & $\mathbf{A}_{3}$ & 2 & 1 relation \\
$v i$ & $\mathbf{A}_{4}$ & 1 & 1 relation \\
\hline
\end{tabular}

TABle 5.3. Del Pezzo surfaces of degree 5

Proposition 5.5. The generalized Del Pezzo surfaces of degree 5 of Table 5.3 can be divided into the following groups:

- Type 0 has a Cox ring with 10 generators and five relations.

- Types ii and iv are toric.

- Types $i, i i i, v, v i$ have a Cox ring with 9 generators and one relation.

Proof. Type 0 has 10 negative curves. By Lemma 2.7. $\operatorname{Cox}(\widetilde{S})$ has at least 10 generators. By Lemma 2.13, this implies that there is more than one relation in $\operatorname{Cox}(\widetilde{S})$. More information on this surface can be found in [Sko93, [Has04, Section 2.2], Bre02]. The toric types are listed in Table 1.4.

For the other types, we calculate the Cox ring in what follows, and we see that each of them has exactly one relation.

Type i $\left(\mathbf{A}_{1}\right)$. The surface of type $i$ has the properties: 
- It is the intersection of the following five quadrics in $\mathbb{P}^{5}$ :

$$
\begin{aligned}
& x_{0} x_{4}-x_{1} x_{2}=x_{0} x_{5}-x_{1} x_{3}=x_{2} x_{5}-x_{3} x_{4} \\
= & x_{1} x_{2}+x_{1} x_{3}+x_{2} x_{3}=x_{1} x_{4}+x_{1} x_{5}+x_{2} x_{5}=0 .
\end{aligned}
$$

- The singularity in $p=(1: 0: 0: 0: 0: 0)$ gives an exceptional divisor $E_{1}$, the seven lines on $S$ are $E_{2}=\left\{x_{1}=x_{2}=x_{4}=x_{5}=0\right\}$, $E_{3}=\left\{x_{1}=x_{3}=x_{4}=x_{5}=0\right\}, E_{4}=\left\{x_{2}=x_{3}=x_{4}=x_{5}=0\right\}$, $E_{5}=\left\{x_{0}=x_{1}=x_{2}=x_{3}=0\right\}, E_{6}=\left\{x_{0}=x_{1}=x_{2}=x_{4}=0\right\}$, $E_{7}=\left\{x_{0}=x_{1}=x_{3}=x_{5}=0\right\}, E_{8}=\left\{x_{0}=x_{2}=x_{3}=x_{4}+x_{5}=\right.$ $0\}$.

- $E_{1}, \ldots, E_{5}$ are a basis of $\operatorname{Pic}(\widetilde{S})$, with $E_{6}=(1,0,1,1,-1), E_{7}=$ $(1,1,0,1,-1), E_{8}=(1,1,1,0,-1),-K_{\widetilde{S}}=(3,2,2,2,-1)$.

- The Cox ring is

$$
\operatorname{Cox}(\widetilde{S})=\mathbb{K}\left[\eta_{1}, \ldots, \eta_{8}\right] /\left(\eta_{2} \eta_{6}+\eta_{3} \eta_{7}+\eta_{4} \eta_{8}\right),
$$

and the relation is of degree $(1,1,1,1,-1)$.

- The extended Dynkin diagram is:

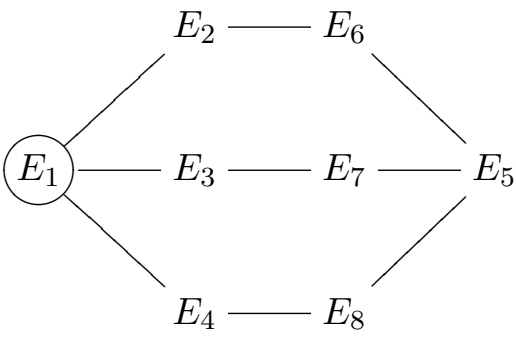

- The anticanonical embedding $S \rightarrow \mathbb{P}^{5}$ is given by

$$
\begin{gathered}
\left(\phi^{*}\left(x_{i}\right)\right)=\left(\eta^{(0,0,0,0,2)} \eta_{6} \eta_{7} \eta_{8}, \eta^{(1,1,1,0,1)} \eta_{6} \eta_{7}, \eta^{(1,1,0,1,1)} \eta_{6} \eta_{8}\right. \\
\left.\eta^{(1,0,1,1,1)} \eta_{7} \eta_{8}, \eta^{(2,2,1,1,0)} \eta_{6}, \eta^{(2,1,2,1,0)} \eta_{7}\right) .
\end{gathered}
$$

We have $\phi^{*}\left(-x_{4}-x_{5}\right)=\eta^{(2,1,1,2,0)} \eta_{8}, \phi^{*}\left(-x_{1}-x_{2}\right)=\eta^{(1,2,0,0,1)} \eta_{6}^{2}$, $\phi^{*}\left(-x_{1}-x_{3}\right)=\eta^{(1,0,2,0,1)} \eta_{7}^{2}, \phi^{*}\left(-x_{2}-x_{3}\right)=\eta^{(1,0,0,2,1)} \eta_{8}^{2}$.

Type iii $\left(\mathbf{A}_{2}\right)$. The surface of type $i i i$ has the properties:

- It is the intersection of the following five quadrics in $\mathbb{P}^{5}$ :

$$
\begin{aligned}
& x_{0} x_{2}-x_{1} x_{5}=x_{0} x_{2}-x_{3} x_{4}=x_{0} x_{3}+x_{1}^{2}+x_{1} x_{4} \\
= & x_{0} x_{5}+x_{1} x_{4}+x_{4}^{2}=x_{3} x_{5}+x_{1} x_{2}+x_{2} x_{4}=0 .
\end{aligned}
$$

- The singularity in $p=(0: 0: 1: 0: 0: 0)$ gives the exceptional divisors $E_{1}, E_{2}$, and the lines are $E_{3}=\left\{x_{0}=x_{1}=x_{3}=x_{4}=0\right\}$, $E_{4}=\left\{x_{0}=x_{1}=x_{4}=x_{5}=0\right\}, E_{5}=\left\{x_{0}=x_{3}=x_{5}=x_{1}+x_{4}=\right.$ $0\}$, and $E_{6}=\left\{x_{2}=x_{3}=x_{5}=x_{1}+x_{4}=0\right\}$.

- A basis of $\operatorname{Pic}(\widetilde{S})$ is $E_{1}, \ldots, E_{5}$, with $E_{6}=(1,0,1,1,-1)$ and $-K_{\widetilde{S}}=(3,2,2,2,1)$.

- The Cox ring is

$$
\operatorname{Cox}(\widetilde{S})=\mathbb{K}\left[\eta_{1}, \ldots, \eta_{6}, \alpha_{1}, \alpha_{2}\right] /\left(\eta_{2} \eta_{5}^{2} \eta_{6}+\eta_{3} \alpha_{1}+\eta_{4} \alpha_{2}\right),
$$


where $\operatorname{deg}\left(\alpha_{1}\right)=A_{1}=(1,1,0,1,1)=\psi^{*}\left(\left\{\left(a^{2}: 0: a b: 0: 0:\right.\right.\right.$ $\left.\left.\left.-b^{2}\right)\right\}\right), \operatorname{deg}\left(\alpha_{2}\right)=A_{2}=(1,1,1,0,1)=\psi^{*}\left(\left\{\left(a^{2}: a b: 0:-b^{2}: 0:\right.\right.\right.$ $0)\})$, and the degree of the relation is $(1,1,1,1,1)$.

- The extended Dynkin diagram is:

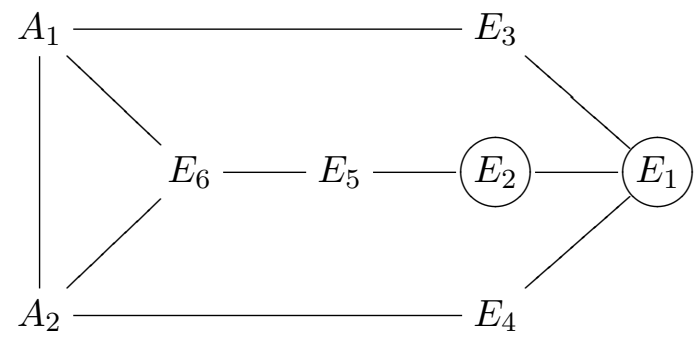

Here, $A_{1}, A_{2}, E_{6}$ meet in $(1: 0: 0: 0: 0: 0)$.

- The anticanonical embedding $\phi: S \rightarrow \mathbb{P}^{5}$ is given by

$$
\begin{aligned}
\left(\phi^{*}\left(x_{i}\right)\right)= & \left(\eta^{(3,2,2,2,1)}, \eta^{(2,1,2,1,0)} \alpha_{1}, \eta_{6} \alpha_{1} \alpha_{2},\right. \\
& \left.\eta^{(1,1,1,0,1)} \eta_{6} \alpha_{1}, \eta^{(2,1,1,2,0)} \alpha_{2}, \eta^{(1,1,0,1,1)} \eta_{6} \alpha_{2}\right),
\end{aligned}
$$

and furthermore, $\phi^{*}\left(-x_{1}-x_{4}\right)=\eta^{(2,2,1,1,2)} \eta_{6}$ and $\phi^{*}\left(-x_{3}-x_{5}\right)=$ $\eta^{(1,2,0,0,3)} \eta_{6}^{2}$.

Type $\mathbf{v}\left(\mathbf{A}_{3}\right)$. The surface of type $v$ has the following properties:

- It is the intersection of the following five quadrics in $\mathbb{P}^{5}$ :

$$
\begin{aligned}
& x_{0} x_{2}-x_{1}^{2}=x_{0} x_{3}-x_{1} x_{4}=x_{2} x_{4}-x_{1} x_{3} \\
= & x_{2} x_{4}+x_{4}^{2}+x_{0} x_{5}=x_{2} x_{3}+x_{3} x_{4}+x_{1} x_{5}=0 .
\end{aligned}
$$

- The singularity is in $p=(0: 0: 0: 0: 0: 1)$, giving three exceptional divisors $E_{1}, E_{2}, E_{3}$. The lines $E_{4}=\left\{x_{0}=x_{1}=x_{2}=\right.$ $\left.x_{4}=0\right\}$ and $E_{5}=\left\{x_{0}=x_{1}=x_{3}=x_{4}=0\right\}$ intersect in $p$.

- A basis of $\operatorname{Pic}(\widetilde{S})$ is $E_{1}, \ldots, E_{5}$, with $-K_{\widetilde{S}}=(2,4,3,2,3)$.

- The Cox ring is

$\operatorname{Cox}(\widetilde{S})=\mathbb{K}\left[\eta_{1}, \ldots, \eta_{5}, \alpha_{1}, \alpha_{2}, \alpha_{3}\right] /\left(\eta_{1} \alpha_{1}^{2}+\eta_{3} \eta_{4}^{2} \alpha_{2}+\eta_{5} \alpha_{3}\right)$,

where $\operatorname{deg}\left(\alpha_{1}\right)=A_{1}=(0,1,1,1,1)=\psi^{*}\left(\left\{\left(a^{2}: 0: 0: 0: a b:\right.\right.\right.$ $\left.\left.\left.-b^{2}\right)\right\}\right), \operatorname{deg}\left(\alpha_{2}\right)=A_{2}=(1,2,1,0,2)=\psi^{*}\left(\left\{\left(a^{2}: a b: b^{2}: 0: 0:\right.\right.\right.$ $0)\}), \operatorname{deg}\left(\alpha_{3}\right)=A_{3}=(1,2,2,2,1)=\psi^{*}\left(\left\{\left(a^{3}: a^{2} b: a b^{2}:-b^{3}:\right.\right.\right.$ $\left.\left.\left.-a b^{2}: 0\right)\right\}\right)$, and the degree of the relation is $(1,2,2,2,2)$.

- The extended Dynkin diagram is:

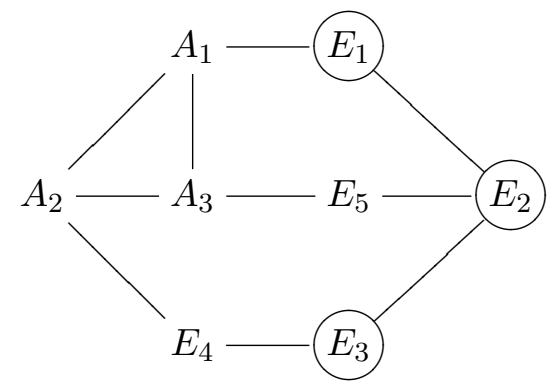

Here, $A_{1}, A_{2}, A_{3}$ meet in $(1: 0: 0: 0: 0: 0)$. 
- The anticanonical embedding $\phi: S \rightarrow \mathbb{P}^{5}$ is given by

$$
\begin{array}{r}
\left(\phi^{*}\left(x_{i}\right)\right)=\left(\eta^{(2,4,3,2,3)}, \eta^{(2,3,2,1,2)} \alpha_{1}, \eta^{(2,2,1,0,1)} \alpha_{1}^{2},\right. \\
\left.\eta^{(1,1,1,1,0)} \alpha_{1} \alpha_{2}, \eta^{(1,2,2,2,1)} \alpha_{2}, \alpha_{2} \alpha_{3}\right),
\end{array}
$$

and furthermore, $\phi^{*}\left(-x_{2}-x_{4}\right)=\eta^{(1,2,1,0,2)} \alpha_{3}$.

Type vi $\left(\mathbf{A}_{4}\right)$. The surface of type $v i$ has the following properties:

- It is given by the intersection of the following five quadrics in $\mathbb{P}^{5}$ :

$$
\begin{aligned}
& x_{0} x_{2}-x_{1}^{2}=x_{0} x_{3}-x_{1} x_{4}=x_{2} x_{4}-x_{1} x_{3} \\
= & x_{1} x_{2}+x_{4}^{2}+x_{0} x_{5}=x_{2}^{2}+x_{3} x_{4}+x_{1} x_{5}=0 .
\end{aligned}
$$

- The singularity is $p=(0: 0: 0: 0: 0: 1)$, giving four exceptional divisors $E_{1}, \ldots, E_{4}$ in $\operatorname{Pic}(\widetilde{S})$, and the line is $E_{5}=\left\{x_{0}=x_{1}=x_{2}=\right.$ $\left.x_{4}=0\right\}$.

- A basis of $\operatorname{Pic}(\widetilde{S})$ is $E_{1}, \ldots, E_{5}$, with $-K_{\widetilde{S}}=(2,4,6,3,5)$.

- The Cox ring is

$\operatorname{Cox}(\widetilde{S})=\mathbb{K}\left[\eta_{1}, \ldots, \eta_{5}, \alpha_{1}, \alpha_{2}, \alpha_{3}\right] /\left(\eta_{1}^{2} \eta_{2} \alpha_{1}^{3}+\eta_{4} \alpha_{2}^{2}+\eta_{5} \alpha_{3}\right)$,

where $\operatorname{deg}\left(\alpha_{1}\right)=A_{1}=(0,1,2,1,2)=\psi^{*}\left(\left\{\left(a^{2}: 0: 0: 0: a b:\right.\right.\right.$

$\left.\left.\left.-b^{2}\right)\right\}\right), \operatorname{deg}\left(\alpha_{2}\right)=A_{2}=(1,2,3,1,3)=\psi^{*}\left(\left\{\left(a^{3}: a^{2} b: a b^{2}: 0: 0:\right.\right.\right.$

$\left.\left.\left.-b^{3}\right)\right\}\right), \operatorname{deg}\left(\alpha_{3}\right)=A_{3}=(2,4,6,3,5)=-K_{\widetilde{S}}=\psi^{*}\left(\left\{\left(a^{5}:-a^{3} b^{2}:\right.\right.\right.$

$\left.\left.\left.a b^{4}:-b^{5}: a^{2} b^{3}: 0\right)\right\}\right)$. The degree of the relation is $(2,4,6,3,6)$.

- The extended Dynkin diagram is:

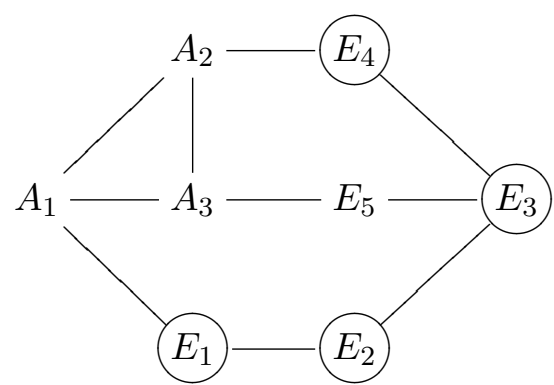

Here, $A_{1}, A_{2}, A_{3}$ meet in $(1: 0: 0: 0: 0: 0)$.

- The anticanonical embedding $\phi: S \rightarrow \mathbb{P}^{5}$ is given by

$$
\begin{gathered}
\left(\phi^{*}\left(x_{i}\right)\right)=\left(\eta^{(2,4,6,3,5)}, \eta^{(2,3,4,2,3)} \alpha_{1}, \eta^{(2,2,2,1,1)} \alpha_{1}^{2},\right. \\
\left.\eta^{(1,1,1,1,0)} \alpha_{1} \alpha_{2}, \eta^{(1,2,3,2,2)} \alpha_{2}, \alpha_{3}\right) .
\end{gathered}
$$

\subsection{Degree 4}

By Remark 1.13 and Remark 1.26, quartic Del Pezzo surfaces are certain intersections of two conics in $\mathbb{P}^{4}$. The extended Dynkin diagrams of their lines and exceptional divisors can be found in [CT88, Proposition 6.1].

Proposition 5.6. The 16 types of generalized Del Pezzo surfaces of degree 4 of Table 5.4 can be divided into the following three groups:

- Types ix, x, xiv are toric.

- The Cox rings of types $v, v i, v i i, x i, x i i, x i i i, x v$ have 9 generators and one relation. 


\begin{tabular}{|c||c|c|c|}
\hline type & singularities & number of lines & type \\
\hline \hline 0 & - & 16 & $\geq 2$ relations \\
$i$ & $\mathbf{A}_{1}$ & 12 & $\geq 2$ relations \\
$i i$ & $2 \mathbf{A}_{1}$ & 9 & $\geq 2$ relations \\
iii & $2 \mathbf{A}_{1}$ & 8 & $\geq 2$ relations \\
$i v$ & $\mathbf{A}_{2}$ & 8 & $\geq 2$ relations \\
$v$ & $3 \mathbf{A}_{1}$ & 6 & 1 relation \\
$v i$ & $\mathbf{A}_{2}+\mathbf{A}_{1}$ & 6 & 1 relation \\
vii & $\mathbf{A}_{3}$ & 5 & 1 relation \\
viii & $\mathbf{A}_{3}$ & 4 & $\geq 2$ relations \\
$i x$ & $4 \mathbf{A}_{1}$ & 4 & toric \\
$x$ & $\mathbf{A}_{2}+2 \mathbf{A}_{1}$ & 4 & toric \\
xi & $\mathbf{A}_{3}+\mathbf{A}_{1}$ & 3 & 1 relation \\
xii & $\mathbf{A}_{4}$ & 3 & 1 relation \\
xiii & $\mathbf{D}_{4}$ & 2 & 1 relation \\
xiv & $\mathbf{A}_{3}+2 \mathbf{A}_{1}$ & 2 & toric \\
xv & $\mathbf{D}_{5}$ & 1 & 1 relation \\
\hline
\end{tabular}

TABLE 5.4. Del Pezzo surfaces of degree 4

- The Cox rings of types $0, i, i i, i i i, i v$, viii have at least 10 generators and at least two independent relations.

Proof. The toric types $i x, x$, xiv are identified using Table 1.4. For types $0, i, i i, i i i, i v$, the number of negative curves is greater than $r+4=9$, so $\operatorname{Cox}(\widetilde{S})$ has more than 9 generators by Lemma 2.7. Therefore, there must be more than one relation.

For type viii, this is not as obvious because it has only 7 negative curves. We can derive the following information from the extended Dynkin diagram of negative curves given in [CT88, Proposition 6.1]:

- The singularity gives the exceptional divisors $E_{1}, E_{2}, E_{3}$, and they intersect the four lines $E_{4}, \ldots, E_{7}$ in the following way:

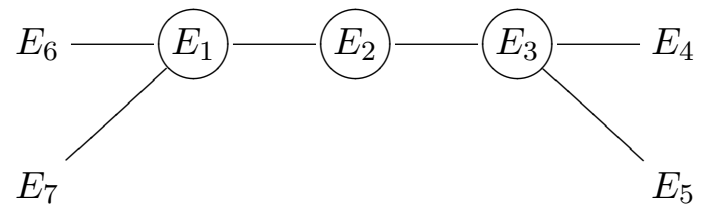

- A basis of $\operatorname{Pic}(\widetilde{S})$ is $E_{1}, \ldots, E_{6}$, with $E_{7}=(-1,0,1,1,1,-1)$ and $-K_{\widetilde{S}}=(1,2,3,2,2,0)$.

- Four of the ten generators of the nef cone are $B_{1}=(1,1,1,0,1,1)$, $B_{2}=(1,1,1,1,0,1), B_{3}=(0,1,2,1,2,-1), B_{4}=(0,1,2,2,1,-1)$, and $\operatorname{dim}\left(H^{0}\left(B_{i}\right)\right)=\chi\left(B_{i}\right)=2$ for $i \in\{1, \ldots, 4\}$.

The subring generated by non-zero sections $\eta_{j} \in H^{0}\left(E_{j}\right)$ for $j \in\{1, \ldots, 7\}$ does not contain two linearly independent sections in any of these degrees $B_{i}$.

Consider a minimal set of generators of $\operatorname{Cox}(\widetilde{S})$. By Lemma 2.8 and since $E_{1}, \ldots, E_{7}$ generate $\Lambda_{\text {eff }}(\widetilde{S})$, it has the form $\eta_{1}, \ldots, \eta_{7}, \alpha_{1}, \ldots, \alpha_{s}$ with 
all $A_{l}:=\operatorname{deg}\left(\alpha_{l}\right)$ nef. For all $i \in\{1, \ldots, 4\}$, it contains two linearly independent sections of degree $B_{l}$, so it contain a section $\beta_{l}$ which is a scalar multiple of $\eta_{1}^{e_{1}} \cdots \eta_{7}^{e_{7}} \alpha_{1}^{a_{1}} \cdots \alpha_{s}^{a_{s}}$ where $e_{j}, a_{l} \geq 0$ and not all $a_{l}$ are zero. Considering their degrees, this means that $\sum_{l=1}^{s} a_{l} A_{l}=B_{i}-\sum_{j=1}^{7} e_{j} E_{j}$, and this degree is nef. However, we calculate directly that the intersection of the nef cone with the negative of the effective cone translated by $B_{i}$ contains only $(0,0,0,0,0,0)$ and $B_{i}$. Consequently, $B_{i}=A_{n}$ for some $n \in\{1, \ldots, s\}$, and all $e_{j}, a_{l}$ are zero except $a_{n}=1$. As this is true for all $i \in\{1, \ldots, 4\}$, the Cox ring must have at least 11 generators.

For the other types, the total number of negative curves is at most $r+3=9$ by the extended Dynkin diagrams of [CT88, and they are not toric. In the following, we calculate their Cox rings, which will show that they have exactly 9 generators and one relation.

Type $\mathbf{v}\left(3 \mathbf{A}_{1}\right)$. Type $v$, which has been considered in [Bro05], has the following properties:

- It is given by the the following quadrics in $\mathbb{P}^{4}$ :

$$
x_{0} x_{1}-x_{2}^{2}=x_{1} x_{2}+x_{2}^{2}+x_{3} x_{4}=0 .
$$

- Its three singularities $p_{1}=(1: 0: 0: 0: 0), p_{2}=(0: 0: 0$ : $1: 0), p_{3}=(0: 0: 0: 0: 1)$ give exceptional divisors $E_{1}, E_{2}, E_{3}$, respectively. The six lines are $E_{4}=\left\{x_{0}=x_{2}=x_{3}=0\right\}, E_{5}=$ $\left\{x_{0}=x_{2}=x_{4}=0\right\}, E_{6}=\left\{x_{1}=x_{2}=x_{3}=0\right\}, E_{7}=\left\{x_{1}=x_{2}=\right.$ $\left.x_{4}=0\right\}, E_{8}=\left\{x_{0}+x_{2}=x_{1}+x_{2}=x_{3}=0\right\}, E_{9}=\left\{x_{0}+x_{2}=\right.$ $\left.x_{1}+x_{2}=x_{4}=0\right\}$

- A basis of $\operatorname{Pic}(\widetilde{S})$ is $E_{1}, \ldots, E_{6}$, with

$E_{7}=(-1,0,0,1,1,-1), E_{8}=(-1,1,-1,1,2,-2), E_{9}=(1,-1,1,0,-1,2)$, and $-K_{\widetilde{S}}=(0,1,1,2,2,0)$.

- The Cox ring is

$$
\operatorname{Cox}(\widetilde{S})=\mathbb{K}\left[\eta_{1}, \ldots, \eta_{9}\right] /\left(\eta_{4} \eta_{5}+\eta_{1} \eta_{6} \eta_{7}+\eta_{8} \eta_{9}\right),
$$

where the relation is of degree $(0,0,0,1,1,0)$.

- The extended Dynkin diagram is:

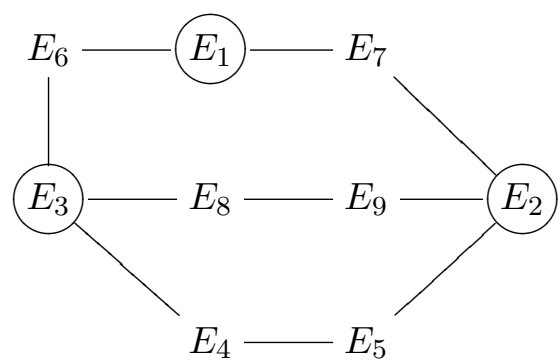

- The anticanonical embedding is given by

$$
\begin{aligned}
\left(\phi^{*}\left(x_{i}\right)\right)= & \left(\eta^{(0,1,1,2,2,0)}, \eta^{(2,1,1,0,0,2)} \eta_{7}^{2}, \eta^{(1,1,1,1,1,1)} \eta_{7},\right. \\
& \left.\eta^{(1,0,2,1,0,2)} \eta_{8}, \eta^{(1,2,0,0,1,0)} \eta_{7}^{2} \eta_{9}\right),
\end{aligned}
$$

and furthermore, $\phi^{*}\left(-x_{0}-x_{2}\right)=\eta^{(0,1,1,1,1,0)} \eta_{8} \eta_{9}, \phi^{*}\left(-x_{1}-x_{2}\right)=$ $\eta^{(1,1,1,0,0,1)} \eta_{7} \eta_{8} \eta_{9}, \phi^{*}\left(\left(x_{0}+x_{2}\right)+\left(x_{1}+x_{2}\right)\right)=\eta^{(0,1,1,0,0,0)} \eta_{8}^{2} \eta_{9}^{2}$. 
Type vi $\left(\mathbf{A}_{2}+\mathbf{A}_{1}\right)$. Type $v i$ has the following properties:

- It is given by the the following quadrics in $\mathbb{P}^{4}$ :

$$
x_{0} x_{1}-x_{2} x_{3}=x_{1} x_{2}+x_{2} x_{4}+x_{3} x_{4}=0 .
$$

- Its singularity $p_{1}=(1: 0: 0: 0: 0)$ gives the exceptional divisors $E_{1}, E_{2}$, and $p_{2}=(0: 0: 0: 0: 1)$ gives $E_{3}$. The six lines are $E_{4}=$ $\left\{x_{0}=x_{2}=x_{3}=0\right\}, E_{5}=\left\{x_{0}=x_{2}=x_{4}=0\right\}, E_{6}=\left\{x_{1}=x_{3}=\right.$ $\left.x_{4}=0\right\}, E_{7}=\left\{x_{1}=x_{2}=x_{4}=0\right\}, E_{8}=\left\{x_{0}=x_{3}=x_{1}+x_{4}=0\right\}$, $E_{9}=\left\{x_{1}=x_{2}=x_{3}=0\right\}$.

- A basis of $\operatorname{Pic}(\widetilde{S})$ is $E_{1}, \ldots, E_{6}$, with $E_{7}=(-1,-2,1,2,1,-2)$, $E_{8}=(-1,-2,1,2,2,-3), E_{9}=(-1,-1,0,1,1,-1)$, and $-K_{\widetilde{S}}=$ $(-1,-2,2,4,3,-3)$.

- The Cox ring is

$$
\operatorname{Cox}(\widetilde{S})=\mathbb{K}\left[\eta_{1}, \ldots, \eta_{9}\right] /\left(\eta_{5} \eta_{7}+\eta_{1} \eta_{3} \eta_{9}^{2}+\eta_{6} \eta_{8}\right),
$$

where the relation is of degree $(-1,-2,1,2,2,-2)$.

- The extended Dynkin diagram is:

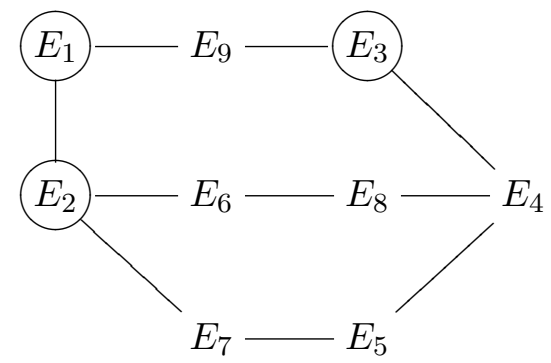

- The anticanonical embedding is given by

$$
\begin{aligned}
\left(\phi^{*}\left(x_{i}\right)\right)= & \left(\eta^{(0,0,1,2,1,0)} \eta_{8}, \eta^{(2,2,1,0,0,1)} \eta_{7} \eta_{9}^{2}, \eta^{(1,1,1,1,1,0)} \eta_{7} \eta_{9}\right. \\
& \left.\eta^{(1,1,1,1,0,1)} \eta_{8} \eta_{9}, \eta^{(1,2,0,0,1,1)} \eta_{7}^{2}\right),
\end{aligned}
$$

and furthermore, $\phi^{*}\left(-x_{1}-x_{4}\right)=\eta^{(1,2,0,0,0,2)} \eta_{7} \eta_{8}$ and $\phi^{*}\left(-x_{2}-\right.$ $\left.x_{3}\right)=\eta^{(2,1,2,1,0,0)} \eta_{9}^{3}$

Type vii $\left(\mathbf{A}_{3}\right)$. Type vii has the following properties:

- It is given by the the following quadrics in $\mathbb{P}^{4}$ :

$$
x_{0} x_{1}-x_{2} x_{3}=x_{2} x_{4}+x_{0} x_{3}+x_{1} x_{3}=0 .
$$

- Its singularity (0:0:0:0:1) gives the exceptional divisors $E_{1}, E_{2}, E_{3}$, and the five lines are $E_{4}=\left\{x_{1}=x_{2}=x_{3}=0\right\}$, $E_{5}=\left\{x_{0}=x_{1}=x_{2}=0\right\}, E_{6}=\left\{x_{0}=x_{2}=x_{3}=0\right\}, E_{7}=\left\{x_{1}=\right.$ $\left.x_{3}=x_{4}=0\right\}, E_{8}=\left\{x_{0}=x_{3}=x_{4}=0\right\}$.

- A basis of $\operatorname{Pic}(\widetilde{S})$ is given by $E_{1}, \ldots, E_{6}$, with $E_{7}=(0,1,1,-1,1,1)$, $E_{8}=(1,1,0,1,1,-1)$, and $-K_{\widetilde{S}}=(2,3,2,1,2,1)$.

- The Cox ring is

$$
\operatorname{Cox}(\widetilde{S})=\mathbb{K}\left[\eta_{1}, \ldots, \eta_{8}, \alpha\right] /\left(\eta_{5} \alpha+\eta_{1} \eta_{4}^{2} \eta_{7}+\eta_{3} \eta_{6}^{2} \eta_{8}\right),
$$

where the relation is of degree $(1,1,1,1,1,1)$, and $\operatorname{deg}(\alpha)=A=$ $(1,1,1,1,0,1)=\psi^{*}\left(\left\{\left(a b:-a b: b^{2}: a^{2}: 0\right)\right\}\right)$ gives the projection $\psi_{1}: x \mapsto\left(x_{3}: x_{4}\right)$ with $\alpha=\psi_{1}^{*}\left(x_{4}\right)$. 
- The extended Dynkin diagram is:

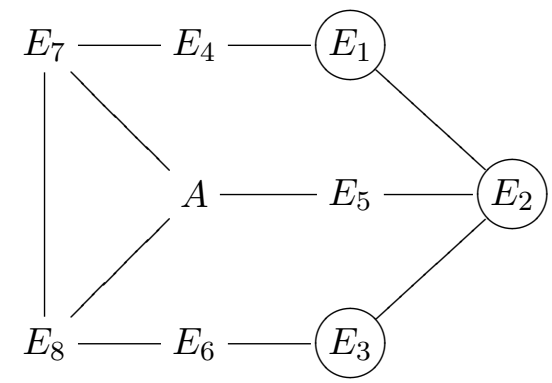

Here, $A, E_{7}, E_{8}$ meet in $(0: 0: 1: 0: 0)$.

- The anticanonical embedding is given by

$$
\begin{gathered}
\left(\phi^{*}\left(x_{i}\right)\right)=\left(\eta^{(2,2,1,2,1,0)} \eta_{7}, \eta^{(1,2,2,0,1,2)} \eta_{8}, \eta^{(2,3,2,1,2,1)}, \eta^{(1,1,1,1,0,1)} \eta_{7} \eta_{8}, \eta_{7} \eta_{8} \alpha\right), \\
\text { and furthermore, } \phi^{*}\left(-x_{0}-x_{1}\right)=\eta^{(1,2,1,0,2,0)} \alpha
\end{gathered}
$$

Type xi $\left(\mathbf{A}_{3}+\mathbf{A}_{1}\right)$. Type $x i$ has the following properties:

- It is given by the the following quadrics in $\mathbb{P}^{4}$ :

$$
x_{0} x_{3}-x_{2} x_{4}=x_{0} x_{1}+x_{1} x_{3}+x_{2}^{2}=0 .
$$

- The singularity $p_{1}=(0: 1: 0: 0: 0)$ gives an exceptional divisor $E_{1}$, and $p_{2}=(0: 0: 0: 0: 1)$ gives $E_{2}, E_{3}, E_{4}$. The three lines are $E_{5}=\left\{x_{0}=x_{2}=x_{3}=0\right\}, E_{6}=\left\{x_{0}=x_{1}=x_{2}=0\right\}$, $E_{7}=\left\{x_{1}=x_{2}=x_{3}=0\right\}$.

- A basis of $\operatorname{Pic}(\widetilde{S})$ is $E_{1}, \ldots, E_{6}$, with $E_{7}=(1,-1,0,1,2,-1)$ and $-K_{\widetilde{S}}=(2,1,2,3,4,0)$.

- The Cox ring is

$\operatorname{Cox}(\widetilde{S})=\mathbb{K}\left[\eta_{1}, \ldots, \eta_{7}, \alpha_{1}, \alpha_{2}\right] /\left(\eta_{6} \alpha_{2}+\eta_{7} \alpha_{1}+\eta_{1} \eta_{3} \eta_{4}^{2} \eta_{5}^{3}\right)$,

where the relation is of degree $(1,0,1,2,3,0), \operatorname{deg}\left(\alpha_{1}\right)=A_{1}=$ $(0,1,1,1,1,1)=\psi^{*}\left(\left\{\left(a^{2}:-b^{2}: a b: 0: 0\right)\right\}\right)$ gives the projection $\psi_{1}: x \mapsto\left(x_{2}: x_{3}\right)$ with $\alpha_{1}=\psi_{1}^{*}\left(x_{3}\right)$, and $\operatorname{deg}\left(\alpha_{2}\right)=A_{2}=$ $(1,0,1,2,3,-1)=\psi^{*}\left(\left\{\left(0: a^{2}: a b:-b^{2}: 0\right)\right\}\right)$ gives the projection $\psi_{2}: x \mapsto\left(x_{0}: x_{2}\right)$ with $\alpha_{2}=\psi_{2}^{*}\left(x_{0}\right)$.

- The extended Dynkin diagram is:

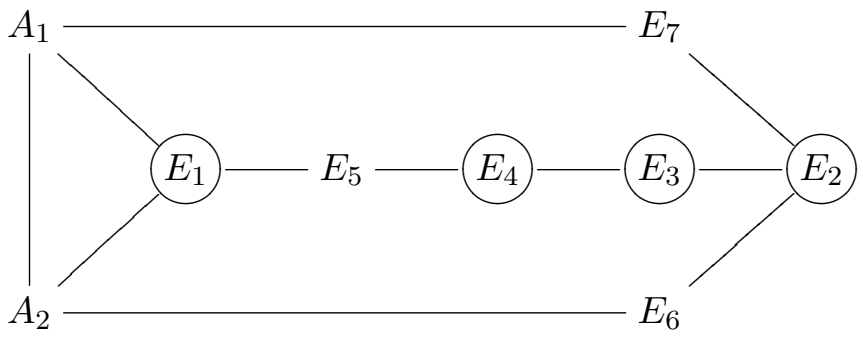

Here, $A_{1}, A_{2}, E_{1}$ meet in one point.

- The anticanonical embedding is given by

$$
\begin{gathered}
\left(\phi^{*}\left(x_{i}\right)\right)=\left(\eta^{(1,1,1,1,1,1)} \alpha_{2}, \eta^{(0,3,2,1,0,2)} \eta_{7}^{2}, \eta^{(1,2,2,2,2,1)} \eta_{7},\right. \\
\left.\eta^{(1,1,1,1,1,0)} \eta_{7} \alpha_{1}, \eta^{(1,0,0,0,0,0)} \alpha_{1} \alpha_{2}\right),
\end{gathered}
$$

and furthermore, $\phi^{*}\left(-x_{0}-x_{3}\right)=\eta^{(2,1,2,3,4,0)}$. 
Type xii $\left(\mathbf{A}_{4}\right)$. Type xii has the following properties:

- It is given by the the following quadrics in $\mathbb{P}^{4}$ :

$$
x_{0} x_{1}-x_{2} x_{3}=x_{0} x_{4}+x_{1} x_{2}+x_{3}^{2}=0 .
$$

- The singularity $p=(0: 0: 0: 0: 1)$ gives the exceptional divisors $E_{1}, \ldots, E_{4}$, and the three lines are $E_{5}=\left\{x_{0}=x_{2}=x_{3}=0\right\}$, $E_{6}=\left\{x_{0}=x_{1}=x_{3}=0\right\}$, and $E_{7}=\left\{x_{1}=x_{3}=x_{4}=0\right\}$.

- A basis of $\operatorname{Pic}(\widetilde{S})$ is given by $E_{1}, \ldots, E_{6}$, with $E_{7}=(1,2,1,0,2,-1)$, and $-K_{\widetilde{S}}=(2,4,3,2,3,1)$.

- The Cox ring is

$$
\operatorname{Cox}(\widetilde{S})=\mathbb{K}\left[\eta_{1}, \ldots, \eta_{7}, \alpha_{1}, \alpha_{2}\right] /\left(\eta_{5} \alpha_{1}+\eta_{1} \alpha_{2}^{2}+\eta_{3} \eta_{4}^{2} \eta_{6}^{3} \eta_{7}\right),
$$

where the degree of the relation is $(1,2,2,2,2,2)$, and $\operatorname{deg}\left(\alpha_{1}\right)=$ $A_{1}=(1,2,2,2,1,2)=\psi^{*}\left(\left\{\left(-b^{3}: a^{3}:-a b^{2}: a^{2} b: 0\right)\right\}\right)$ gives the projection $\psi_{1}$ from $E_{7}$, with $\alpha_{1}=\psi_{1}^{*}\left(x_{4}\right)$, and $\operatorname{deg}\left(\alpha_{2}\right)=A_{2}=$ $(0,1,1,1,1,1)=\psi^{*}\left(\left\{\left(a^{2}: 0: 0:-a b: b^{2}\right)\right\}\right)$ gives the projection $\psi_{2}: x \mapsto\left(x_{1}: x_{3}\right)$ with $\alpha_{2}=\psi_{2}^{*}\left(x_{1}\right)$.

- The extended Dynkin diagram is:

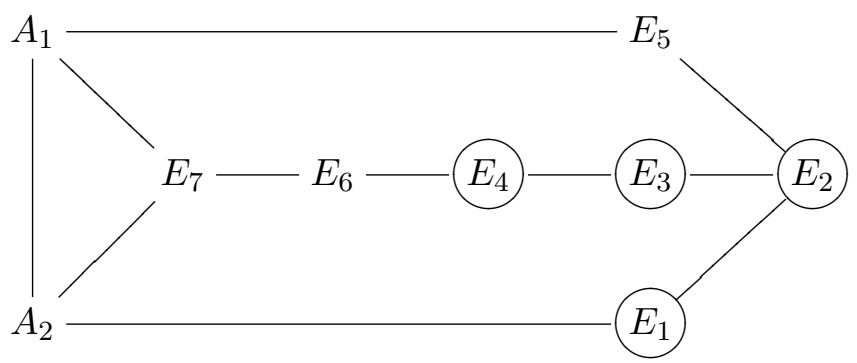

Here, $A_{1}, A_{2}, E_{7}$ meet in $(1: 0: 0: 0: 0)$.

- The anticanonical embedding is given by

$$
\left(\phi^{*}\left(x_{i}\right)\right)=\left(\eta^{(2,4,3,2,3,1)}, \eta^{(1,1,1,1,0,1)} \eta_{7} \alpha_{2}, \eta^{(2,3,2,1,2,0)} \alpha_{2}, \eta^{(1,2,2,2,1,2)} \eta_{7}, \eta_{7} \alpha_{1}\right) .
$$

Type xiii $\left(\mathbf{D}_{4}\right)$. Type xiii, which is considered in more detail in Chapter 10 (see [BB05 for a form whose lines are not defined over $\mathbb{Q}$ ), has the following properties:

- It is given by the the following quadrics in $\mathbb{P}^{4}$ :

$$
x_{0} x_{3}-x_{1} x_{4}=x_{0} x_{1}+x_{1} x_{3}+x_{2}^{2}=0 .
$$

- The singularity $p=(0: 0: 0: 0: 1)$ gives four exceptional divisor $E_{1}, \ldots, E_{4}$, and its lines are $E_{5}=\left\{x_{0}=x_{1}=x_{2}=0\right\}$ and $E_{6}=$ $\left\{x_{1}=x_{2}=x_{3}=0\right\}$.

- A basis of $\operatorname{Pic}(\widetilde{S})$ is given by $E_{1}, \ldots, E_{6}$, and $-K_{\widetilde{S}}=(4,2,3,3,2,2)$.

- The Cox ring is

$\operatorname{Cox}(\widetilde{S})=\mathbb{K}\left[\eta_{1}, \ldots, \eta_{6}, \alpha_{1}, \alpha_{2}, \alpha_{3}\right] /\left(\eta_{3} \eta_{5}^{2} \alpha_{2}+\eta_{4} \eta_{6}^{2} \alpha_{3}+\eta_{2} \alpha_{1}^{2}\right)$,

where the degree of the relation is $(2,1,2,2,2,2), \operatorname{deg}\left(\alpha_{1}\right)=A_{1}=$ $(1,0,1,1,1,1)=\psi^{*}\left(\left\{\left(a b: b^{2}: 0:-a b:-a^{2}\right)\right\}\right)$ gives the projection $\psi_{1}: x \mapsto\left(x_{1}: x_{2}\right)$ with $\alpha_{1}=\psi_{1}^{*}\left(x_{2}\right), \operatorname{deg}\left(\alpha_{2}\right)=A_{2}=$ $(2,1,1,2,0,2)=\psi^{*}\left(\left\{\left(0: a^{2}: a b:-b^{2}: 0\right)\right\}\right)$ gives the projection $\psi_{2}: x \mapsto\left(x_{0}: x_{1}\right)$ with $\alpha_{2}=\psi_{2}^{*}\left(x_{0}\right)$, and $\operatorname{deg}\left(\alpha_{3}\right)=A_{3}=$ 
$(2,1,2,1,2,0)=\psi^{*}\left(\left\{\left(a^{2}:-b^{2}: a b: 0: 0\right)\right\}\right)$ gives the projection $\psi_{3}: x \mapsto\left(x_{1}: x_{3}\right)$ with $\alpha_{3}=\psi_{3}^{*}\left(x_{3}\right)$.

- The extended Dynkin diagram is:

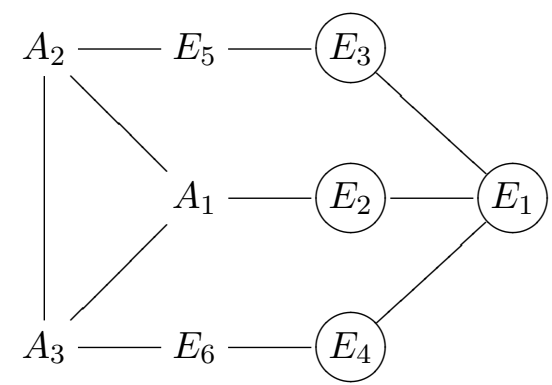

Here, $A_{1}, A_{2}, A_{3}$ intersect in $(0: 1: 0: 0: 0)$.

- The anticanonical embedding is given by

$$
\left(\phi^{*}\left(x_{i}\right)\right)=\left(\eta^{(2,1,2,1,2,0)} \alpha_{2}, \eta^{(4,2,3,3,2,2)}, \eta^{(3,2,2,2,1,1)} \alpha_{1}, \eta^{(2,1,1,2,0,2)} \alpha_{3}, \alpha_{2} \alpha_{3}\right) .
$$

Furthermore, $\phi^{*}\left(-x_{0}-x_{3}\right)=\eta^{(2,2,1,1,0,0)} \alpha_{1}^{2}$.

Type xv $\left(\mathbf{D}_{5}\right)$. Type $x v$, which has already been considered in [BB04, has the following properties:

- It is given by the the following quadrics in $\mathbb{P}^{4}$ :

$$
x_{0} x_{1}-x_{2}^{2}=x_{3}^{2}+x_{0} x_{4}+x_{1} x_{2}=0
$$

- The singularity $p=(0: 0: 0: 0: 1)$ gives five exceptional divisor $E_{1}, \ldots, E_{5}$, and its line is $E_{6}=\left\{x_{0}=x_{2}=x_{3}=0\right\}$.

- A basis of $\operatorname{Pic}(\widetilde{S})$ is given by $E_{1}, \ldots, E_{6}$, and $-K_{\widetilde{S}}=(6,5,3,4,2,4)$.

- The Cox ring is

$\operatorname{Cox}(\widetilde{S})=\mathbb{K}\left[\eta_{1}, \ldots, \eta_{6}, \alpha_{1}, \alpha_{2}, \alpha_{3}\right] /\left(\eta_{3} \alpha_{1}^{2}+\eta_{2} \eta_{6}^{2} \alpha_{3}+\eta_{4} \eta_{5}^{2} \alpha_{2}^{3}\right)$,

where the degree of the relation is $(6,6,3,4,2,6)$, and $\operatorname{deg}\left(\alpha_{1}\right)=$ $A_{1}=(3,3,1,2,1,3)=\psi^{*}\left(\left\{\left(a^{3}: a b^{2}: a^{2} b: 0:-a^{3}\right)\right\}\right)$ gives the projection $\psi_{1}$ from $E_{6}$ with $\alpha_{1}=\psi_{1}^{*}\left(x_{3}\right), \operatorname{deg}\left(\alpha_{2}\right)=A_{2}=$ $(2,2,1,1,0,2)=\psi^{*}\left(\left\{\left(a^{2}: 0: 0: a b:-b^{2}\right)\right\}\right)$ gives the projection $\psi_{2}: x \mapsto\left(x_{0}: x_{2}\right)$ with $\alpha_{2}=\psi_{2}^{*}\left(x_{2}\right)$, and $\operatorname{deg}\left(\alpha_{3}\right)=A_{3}=$ $-K_{\widetilde{S}}=\psi^{*}\left(\left\{\left(-a^{4}:-b^{4}: a^{2} b^{2}: a b^{3}: 0\right)\right\}\right)$ gives the anticanonical embedding $\phi$ with $\alpha_{3}=\phi^{*}\left(x_{4}\right)$.

- The extended Dynkin diagram is:

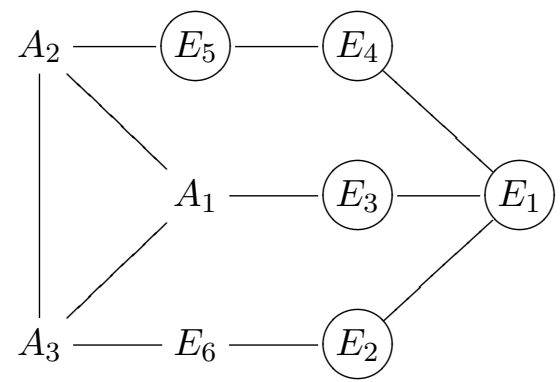

where $A_{1}, A_{2}, A_{3}$ intersect in $(1: 0: 0: 0: 0)$.

- For the anticanonical embedding,

$$
\left(\phi^{*}\left(x_{i}\right)\right)=\left(\eta^{(6,5,3,4,2,4)}, \eta^{(2,1,1,2,2,0)} \alpha_{2}^{2}, \eta^{(4,3,2,3,2,2)} \alpha_{2}, \eta^{(3,2,2,2,1,1)} \alpha_{1}, \alpha_{3}\right) \text {. }
$$




\subsection{Degree 3}

The classification of cubic Del Pezzo surfaces is classical and goes back to Schläfli [Sch63. Together with their number of lines, the list in Table 5.5 can be found in BW79.

\begin{tabular}{|c||c|c|c|}
\hline type & singularities & number of lines & type \\
\hline \hline 0 & - & 27 & $\geq 2$ relations \\
$i$ & $\mathbf{A}_{1}$ & 21 & $\geq 2$ relations \\
$i i$ & $2 \mathbf{A}_{1}$ & 16 & $\geq 2$ relations \\
$i i i$ & $\mathbf{A}_{2}$ & 15 & $\geq 2$ relations \\
$i v$ & $3 \mathbf{A}_{1}$ & 12 & $\geq 2$ relations \\
$v$ & $\mathbf{A}_{2}+\mathbf{A}_{1}$ & 11 & $\geq 2$ relations \\
vi & $\mathbf{A}_{3}$ & 10 & $\geq 2$ relations \\
vii & $4 \mathbf{A}_{1}$ & 9 & $\geq 2$ relations \\
viii & $\mathbf{A}_{2}+2 \mathbf{A}_{1}$ & 8 & $\geq 2$ relations \\
ix & $\mathbf{A}_{3}+\mathbf{A}_{1}$ & 7 & $\geq 2$ relations \\
$x$ & $2 \mathbf{A}_{2}$ & 7 & $\geq 2$ relations \\
xi & $\mathbf{A}_{4}$ & 6 & $\geq 2$ relations \\
xii & $\mathbf{D}_{4}$ & 6 & 1 relation \\
xiii & $\mathbf{A}_{3}+2 \mathbf{A}_{1}$ & 5 & 1 relation \\
xiv & $2 \mathbf{A}_{2}+\mathbf{A}_{1}$ & 5 & 1 relation \\
xv & $\mathbf{A}_{4}+\mathbf{A}_{1}$ & 4 & 1 relation \\
xvi & $\mathbf{A}_{5}$ & 3 & $\geq 2$ relations \\
xvii & $\mathbf{D}_{5}$ & 3 & 1 relation \\
xviii & $3 \mathbf{A}_{2}$ & 3 & toric \\
xix & $\mathbf{A}_{5}+\mathbf{A}_{1}$ & 2 & 1 relation \\
xx & $\mathbf{E}_{6}$ & 1 & 1 relation \\
\hline
\end{tabular}

TABle 5.5. Del Pezzo surfaces of degree 3

Proposition 5.7. The cubic Del Pezzo surfaces of Table 5.5 belong to the following groups:

- Type xviii is toric.

- The Cox rings of types xii, xiii, xiv, xv, xvii, xix, xx have 10 generators and one relation.

- The Cox rings of types $0, i, i i, i i i, i v, v, v i, v i i, v i i i, i x, x, x i, x v i$ have at least 11 generators and at least two independent relations.

Proof. By Table 1.4, type xviii is the only toric cubic Del Pezzo surface. It has been studied extensively, for example in [Bre98], Fou98, HBM99.

Using Lemma 2.7 and Lemma 2.13, and since the number of negative curves is at least 11 , types 0 to $x$ must have more than one relation. For types $x i, x v i$, this is not as obvious because the number of negative curves is 10 and 8 , respectively.

For type $x i$, the negative curves give 10 necessary generators of the Cox ring. The extended Dynkin diagram of the exceptional divisors $E_{1}, \ldots, E_{4}$ 
and the six lines $E_{5}, \ldots, E_{10}$ is:

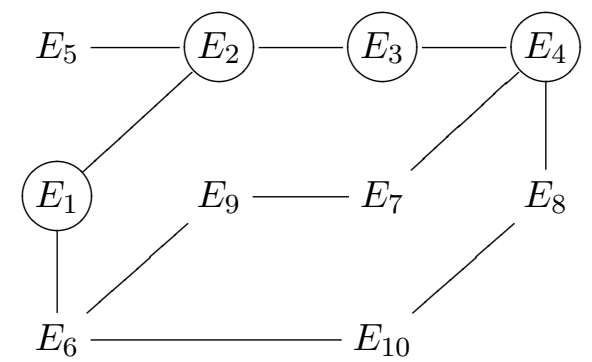

A basis of $\operatorname{Pic}(\widetilde{S})$ is given by $E_{1}, \ldots, E_{7}$, and in terms of this basis,

$$
\begin{aligned}
E_{8} & =(1,1,0,-1,1,1,-1), \quad E_{9}=(1,2,1,0,2,0,-1), \\
E_{10} & =(0,1,1,1,1,-1,1), \quad-K_{\widetilde{S}}=(2,3,2,1,2,1,0) .
\end{aligned}
$$

The divisors $E_{1}, \ldots, E_{10}$ generate the effective cone. We check that $A=$ $(1,1,1,1,0,1,1)$ is nef. Therefore, we calculate $\operatorname{dim} H^{0}(A)=\chi(A)=2$ by Riemann-Roch. However, the subring generated by non-zero sections of $H^{0}\left(E_{i}\right)$ does not give two linearly independent sections in $H^{0}(A)$. Hence, $\operatorname{Cox}(\widetilde{S})$ must have more than 10 generators.

For type $x v i$, we have the following information:

- The singularity gives the exceptional divisors $E_{1}, \ldots, E_{5}$, and there are three lines $E_{6}, E_{7}, E_{8}$.

- The extended Dynkin diagram of negative curves is:

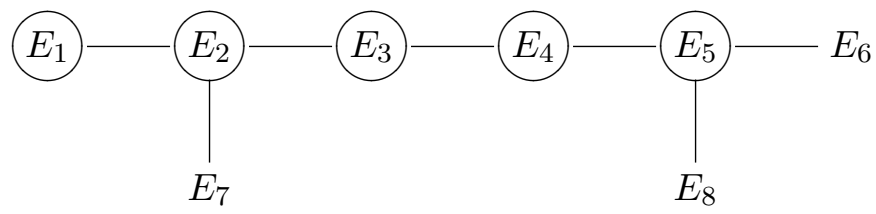

- A basis of $\operatorname{Pic}(\widetilde{S})$ is $E_{1}, \ldots, E_{7}$, with $E_{8}=(1,2,1,0,-1,-1,2)$ and $-K_{\widetilde{S}}=(2,4,3,2,1,0,3)$.

- Three of the 13 generators of the nef cone are $B_{1}=(0,1,1,1,1,1)$, $B_{2}=(1,3,2,1,0,-1,3), B_{3}=(1,2,2,2,2,2,1)$, with

$$
\operatorname{dim}\left(H^{0}\left(B_{1}\right)\right)=\operatorname{dim}\left(H^{0}\left(B_{2}\right)\right)=2, \quad \operatorname{dim}\left(H^{0}\left(B_{3}\right)\right)=3 .
$$

As for type viii of degree 4 , we check that a minimal system of generators of $\operatorname{Cox}(\widetilde{S})$ can be assumed to contain $\eta_{1}, \ldots, \eta_{8}$ with non-zero $\eta_{j} \in H^{0}\left(E_{j}\right)$, and $\beta_{1}, \beta_{2}$ of degree $B_{1}, B_{2}$, respectively. However, it is not hard to check that the subring of $\operatorname{Cox}(\widetilde{S})$ generated by $\eta_{1}, \ldots, \eta_{8}, \beta_{1}, \beta_{2}$ contains only two linearly independent sections in degree $B_{3}$. Therefore, $\operatorname{Cox}(\widetilde{S})$ must have more than 10 generators.

For each type whose Cox ring has exactly 10 generators, the calculations follow below.

REMARK 5.8. By BW79], some types of cubic surfaces do not have a single normal form, but a family with one or more parameters. More precisely, this happens exactly for the types $0, i, i i, i i i, i v, v, v i, x$. Furthermore, by [BW79, Lemma 4], the $\mathbf{D}_{4}$ cubic surface (type $x i i$ ) is the only surface which has more than one normal form, but not a family. The two different surfaces with a $\mathbf{D}_{4}$ singularity are also discussed in [HT04, Remark 4.1]. 
Type xii $\left(\mathbf{D}_{4}\right)$. As mentioned above, type xii has two different forms:

- The first is given by

$$
x_{0}\left(x_{1}+x_{2}+x_{3}\right)^{2}-x_{1} x_{2} x_{3}=0,
$$

the second by

$$
x_{0}\left(x_{1}+x_{2}+x_{3}\right)^{2}+x_{1} x_{2}\left(x_{1}+x_{2}\right)=0 .
$$

- The singularity is $p=(1: 0: 0: 0)$, giving four exceptional divisors $E_{1}, \ldots, E_{4}$. In the first form, the six lines are $E_{5}=\left\{x_{1}=x_{2}+\right.$ $\left.x_{3}=0\right\}, E_{6}=\left\{x_{2}=x_{1}+x_{3}=0\right\}, E_{7}=\left\{x_{3}=x_{1}+x_{2}=0\right\}$, $E_{8}=\left\{x_{0}=x_{1}=0\right\}, E_{9}=\left\{x_{0}=x_{2}=0\right\}, E_{10}=\left\{x_{0}=x_{3}=0\right\}$. In the second form, $E_{10}$ is replaced by $\left\{x_{0}=x_{1}+x_{2}=0\right\}$.

- A basis of $\operatorname{Pic}(\widetilde{S})$ is given by $E_{1}, \ldots, E_{7}$, with

$$
\begin{aligned}
E_{8} & =(1,0,1,1,-1,1,1), & & E_{9}=(1,1,0,1,1,-1,1), \\
E_{10} & =(1,1,1,0,1,1,-1), & & -K_{\widetilde{S}}=(3,2,2,2,1,1,1) .
\end{aligned}
$$

- The Cox ring is

$\operatorname{Cox}(\widetilde{S})=\mathbb{K}\left[\eta_{1}, \ldots, \eta_{10}\right] /\left(\eta_{2} \eta_{5}^{2} \eta_{8}+\eta_{3} \eta_{6}^{2} \eta_{9}+\eta_{4} \eta_{7}^{2} \eta_{10}-A \cdot \eta_{1} \eta_{2} \eta_{3} \eta_{4} \eta_{5} \eta_{6} \eta_{7}\right)$, where the constant $A$ is 1 for the first form and 0 for the second form, and the degree of the relation is $(1,1,1,1,1,1,1)$.

- The extended Dynkin diagram is:

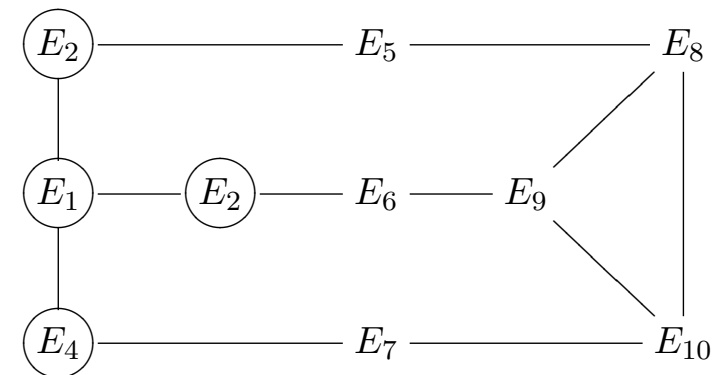

The lines $E_{8}, E_{9}, E_{10}$ meet in one point only in case of the second form.

- For the first form, the anticanonical embedding $\phi: S \rightarrow \mathbb{P}^{3}$ is given by

$$
\left(\phi^{*}\left(x_{i}\right)\right)=\left(\eta_{8} \eta_{9} \eta_{10}, \eta^{(2,2,1,1,2,0,0)} \eta_{8}, \eta^{(2,1,2,1,0,2,0)} \eta_{9}, \eta^{(2,1,1,2,0,0,2)} \eta_{10}\right),
$$

and furthermore, $\phi^{*}\left(x_{1}+x_{2}+x_{3}\right)=\eta^{(3,2,2,2,1,1,1)}$. The second form differs from this as follows: $\phi^{*}\left(-x_{1}-x_{2}\right)=\eta^{(2,1,1,2,0,0,2)} \eta_{10}$, and $\phi^{*}\left(x_{3}\right)=\eta^{(3,2,2,2,1,1,1)}+\eta^{(2,1,1,2,0,0,2)} \eta_{10}$.

Type xiii $\left(\mathbf{A}_{3}+2 \mathbf{A}_{1}\right)$. Type xiii has the following properties:

- It is given by the following cubic in $\mathbb{P}^{3}$ :

$$
x_{3}^{2}\left(x_{1}+x_{2}\right)+x_{0} x_{1} x_{2}=0 .
$$

- The singularities in $p_{1}=(0: 1: 0: 0)$ and $p_{2}=(0: 0: 1: 0)$ give exceptional divisors $E_{1}, E_{10}$, respectively, and the singularity in $p_{3}=(1: 0: 0: 0)$ gives $E_{2}, E_{3}, E_{4}$. The five lines are given by $E_{5}=\left\{x_{0}=x_{3}=0\right\}, E_{6}=\left\{x_{1}=x_{3}=0\right\}, E_{7}=\left\{x_{2}=x_{3}=0\right\}$, $E_{8}=\left\{x_{1}=x_{2}=0\right\}, E_{9}=\left\{x_{0}=x_{1}+x_{2}=0\right\}$. 
- A basis of the $\operatorname{Pic}(\widetilde{S})$ is $E_{1}, \ldots, E_{7}$, and $E_{8}=(1,0,-1,-1,1,-1,1)$, $E_{9}=(0,1,1,1,-1,1,1), E_{10}=(1,1,0,-1,0,-2,2)$, and $-K_{\widetilde{S}}=$ $(2,2,1,0,1,-1,3)$.

- The Cox ring is

$$
\operatorname{Cox}(\widetilde{S})=\mathbb{K}\left[\eta_{1}, \ldots, \eta_{10}\right] /\left(\eta_{4} \eta_{6}^{2} \eta_{10}+\eta_{1} \eta_{2} \eta_{7}^{2}+\eta_{8} \eta_{9}\right),
$$

where the relation is of degree $(1,1,0,0,0,0,2)$.

- The extended Dynkin diagram is:

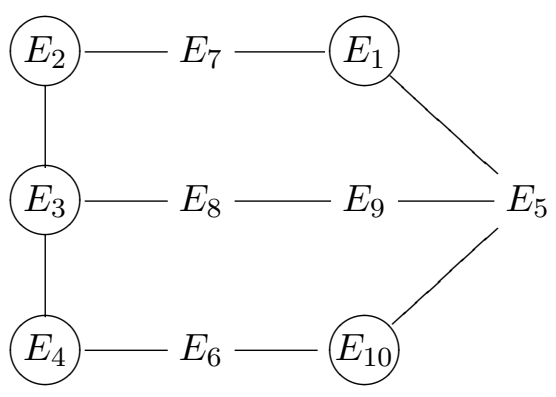

- For the anticanonical embedding,

$$
\begin{gathered}
\left(\phi^{*}\left(x_{i}\right)\right)=\left(\eta^{(1,0,0,0,2,0,0)} \eta_{9} \eta_{10}, \eta^{(0,1,2,2,0,2,0)} \eta_{8} \eta_{10},\right. \\
\left.\eta^{(1,2,2,1,0,0,2)} \eta_{8}, \eta^{(1,1,1,1,1,1,1)} \eta_{10}\right),
\end{gathered}
$$

and furthermore, $\phi^{*}\left(-x_{1}-x_{2}\right)=\eta^{(0,1,2,1,0,0,0)} \eta_{8}^{2} \eta_{9}$.

Type xiv $\left(2 \mathbf{A}_{2}+\mathbf{A}_{1}\right)$. Type xiv has the following properties:

- Its equation is

$$
x_{3}^{2}\left(x_{1}+x_{3}\right)+x_{0} x_{1} x_{2}=0 .
$$

- The singularity $(0: 1: 0: 0)$ gives an exceptional divisor $E_{1}$, and $(1: 0: 0: 0)$ gives $E_{2}, E_{3}$, and $(0: 0: 1: 0)$ gives $E_{4}, E_{5}$. The lines are $E_{6}=\left\{x_{0}=x_{3}=0\right\}, E_{7}=\left\{x_{1}=x_{3}=0\right\}, E_{8}=\left\{x_{2}=x_{3}=0\right\}$, $E_{9}=\left\{x_{0}=x_{1}+x_{3}=0\right\}, E_{10}=\left\{x_{2}=x_{1}+x_{3}=0\right\}$.

- A basis of $\operatorname{Pic}(\widetilde{S})$ is $E_{1}, \ldots, E_{7}$, and $E_{8}=(-1,0,1,0,1,-1,2), E_{9}=$ $(-1,1,2,-1,1,-2,3), E_{10}=(1,-1,-1,1,0,2,-1)$, and $-K_{\widetilde{S}}=$ $(0,1,2,1,2,0,3)$.

- The Cox ring is

$$
\operatorname{Cox}(\widetilde{S})=\mathbb{K}\left[\eta_{1}, \ldots, \eta_{10}\right] /\left(\eta_{3} \eta_{5} \eta_{7}^{2}+\eta_{1} \eta_{6} \eta_{8}+\eta_{9} \eta_{10}\right),
$$

where the relation is of degree $(0,0,1,0,1,0,2)$.

- The extended Dynkin diagram is:

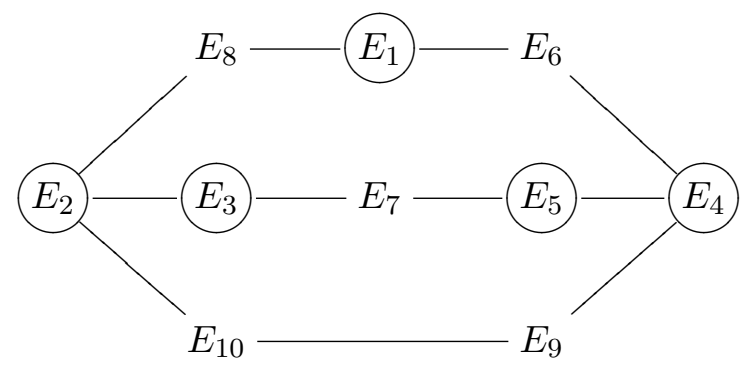


- For the anticanonical embedding,

$$
\begin{gathered}
\left(\phi^{*}\left(x_{i}\right)\right)=\left(\eta^{(1,0,0,2,1,2,0)} \eta_{9}, \eta^{(0,1,2,1,2,0,3)}, \eta^{(1,2,1,0,0,0,0)} \eta_{8}^{2} \eta_{10}, \eta^{(1,1,1,1,1,1,1)} \eta_{8}\right), \\
\text { and furthermore, } \phi^{*}\left(-x_{1}-x_{3}\right)=\eta^{(0,1,1,1,1,0,1)} \eta_{9} \eta_{10} .
\end{gathered}
$$

Type $\mathbf{x v}\left(\mathbf{A}_{4}+\mathbf{A}_{1}\right)$. Type $x v$ has the following properties:

- Its equation is

$$
x_{2} x_{3}^{2}+x_{1}^{2} x_{3}+x_{0} x_{1} x_{2}=0 .
$$

- The singularity $(0: 0: 1: 0)$ gives an exceptional divisor $E_{9}$, and $(1: 0: 0: 0)$ gives $E_{1}, \ldots, E_{4}$. The lines are $E_{5}=\left\{x_{0}=x_{3}=0\right\}$, $E_{6}=\left\{x_{1}=x_{3}=0\right\}, E_{7}=\left\{x_{1}=x_{2}=0\right\}, E_{8}=\left\{x_{2}=x_{3}=0\right\}$.

- A basis of $\operatorname{Pic}(\widetilde{S})$ is $E_{1}, \ldots, E_{7}$, and $E_{8}=(0,1,1,1,-1,1,1), E_{9}=$ $(1,2,1,0,-1,-1,2)$, and $-K_{\widetilde{S}}=(2,4,3,2,-1,1,3)$.

- The Cox ring is

$$
\operatorname{Cox}(\widetilde{S})=\mathbb{K}\left[\eta_{1}, \ldots, \eta_{9}, \alpha\right] /\left(\eta_{1} \eta_{5} \eta_{8}^{2}+\eta_{3} \eta_{4}^{2} \eta_{6}^{3} \eta_{9}+\eta_{7} \alpha\right),
$$

where the relation is of degree $(1,2,2,2,-1,2,2)$, and $\operatorname{deg}(\alpha)=$ $A=(1,2,2,2,-1,2,1)=\psi^{*}\left(\left\{\left(0: a b: a^{2}:-b^{2}\right)\right\}\right)$ describes the projection $\psi_{1}$ from $E_{5}$, with $\alpha=\psi_{1}^{*}\left(x_{0}\right)$.

- The extended Dynkin diagram is:

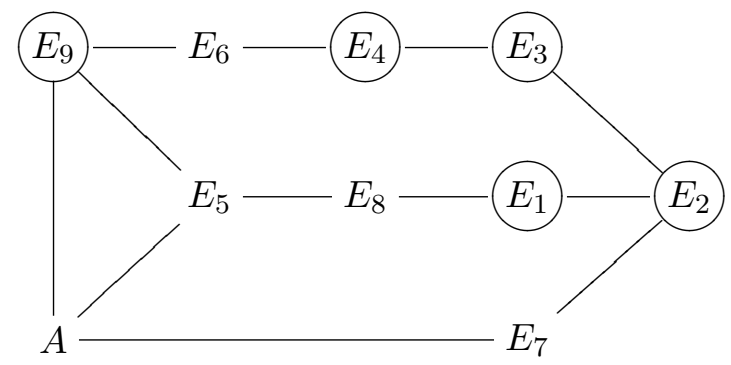

Here, $A, E_{5}, E_{9}$ meet in one point.

- The anticanonical embedding is given by

$$
\left(\phi^{*}\left(x_{i}\right)\right)=\left(\eta_{5} \eta_{9} \alpha, \eta^{(1,2,2,2,0,2,1)} \eta_{9}, \eta^{(2,3,2,1,0,0,2)} \eta_{8}, \eta^{(1,1,1,1,1,1,0)} \eta_{8} \eta_{9}\right) .
$$

Type xvii $\left(\mathbf{D}_{5}\right)$. Type xvii has the following properties:

- Its equation is

$$
x_{3} x_{0}^{2}+x_{0} x_{2}^{2}+x_{1}^{2} x_{2}=0 .
$$

- The singularity $(0: 0: 0: 1)$ gives exceptional divisors $E_{1}, \ldots, E_{5}$. The lines are $E_{6}=\left\{x_{0}=x_{1}=0\right\}, E_{7}=\left\{x_{0}=x_{2}=0\right\}$, and $E_{8}=\left\{x_{2}=x_{3}=0\right\}$.

- A basis of $\operatorname{Pic}(\widetilde{S})$ is $E_{1}, \ldots, E_{7}$, with $E_{8}=(2,2,1,1,0,2,-1)$ and $-K_{\widetilde{S}}=(4,3,2,3,2,2,1)$.

- The Cox ring is

$\operatorname{Cox}(\widetilde{S})=\mathbb{K}\left[\eta_{1}, \ldots, \eta_{8}, \alpha_{1}, \alpha_{2}\right] /\left(\eta_{2} \eta_{6}^{2} \alpha_{2}+\eta_{4} \eta_{5}^{2} \eta_{7}^{3} \eta_{8}+\eta_{3} \alpha_{1}^{2}\right)$,

where the relation has degree $(2,2,1,2,2,2,2)$, and $\operatorname{deg}\left(\alpha_{1}\right)=A_{1}=$ $(1,1,0,1,1,1,1)=\psi^{*}\left(\left\{\left(a^{2}: 0: a b:-b^{2}\right)\right\}\right)$ describes a projection $\psi_{1}$ from $E_{6}$ with $\alpha_{1}=\psi_{1}^{*}\left(x_{1}\right), \operatorname{deg}\left(\alpha_{2}\right)=A_{2}=(2,1,1,2,2,0,2)=$ 
$\psi^{*}\left(\left\{\left(a^{2}: a b:-b^{2}: 0\right)\right\}\right)$ describes the projection $\psi_{2}$ from $E_{8}$ with $\alpha_{2}=\psi_{2}^{*}\left(x_{3}\right)$.

- The extended Dynkin diagram is:

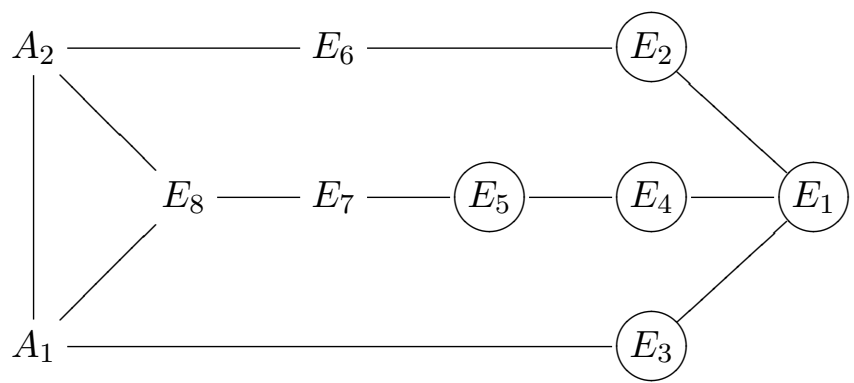

Here, $A_{1}, A_{2}, E_{8}$ meet in $(1: 0: 0: 0)$.

- The anticanonical embedding is given by

$\left(\phi^{*}\left(x_{i}\right)\right)=\left(\eta^{(4,3,2,3,2,2,1)}, \eta^{(3,2,2,2,1,1,0)} \alpha_{1}, \eta^{(2,1,1,2,2,0,2)} \eta_{8}, \eta_{8} \alpha_{2}\right)$.

Type xix $\left(\mathbf{A}_{5}+\mathbf{A}_{1}\right)$. Type $x i x$ has the following properties:

- Its equation is

$$
x_{1}^{3}+x_{2} x_{3}^{2}+x_{0} x_{1} x_{2}=0 .
$$

- The singularity $(0: 0: 1: 0)$ gives an exceptional divisor $E_{8}$, and $(1: 0: 0: 0)$ gives $E_{1}, \ldots, E_{5}$. The lines are $E_{6}=\left\{x_{1}=x_{2}=0\right\}$ and $E_{7}=\left\{x_{1}=x_{3}=0\right\}$.

- A basis of $\operatorname{Pic}(\widetilde{S})$ is $E_{1}, \ldots, E_{7}$, with $E_{8}=(-1,0,1,2,1,2,-2)$ and $-K_{\widetilde{S}}=(1,2,3,4,2,3,0)$.

- The Cox ring is

$\operatorname{Cox}(\widetilde{S})=\mathbb{K}\left[\eta_{1}, \ldots, \eta_{8}, \alpha_{1}, \alpha_{2}\right] /\left(\eta_{1}^{3} \eta_{2}^{2} \eta_{3} \eta_{7}^{4} \eta_{8}+\eta_{5} \alpha_{1}^{2}+\eta_{6} \alpha_{2}\right)$,

where the relation is of degree $(2,2,2,2,1,2,2)$, and $\operatorname{deg}\left(\alpha_{1}\right)=$ $A_{1}=(1,1,1,1,0,1,1)=\psi^{*}\left(\left\{\left(a^{2}: a b:-b^{2}: 0\right)\right\}\right)$ (which describes the projection $\psi_{1}$ from $E_{7}$, with $\left.\alpha_{1}=\psi_{1}^{*}\left(x_{3}\right)\right)$, and $\operatorname{deg}\left(\alpha_{2}\right)=A_{2}=$ $(2,2,2,2,1,1,2)=\psi^{*}\left(\left\{\left(0: a b^{2}:-a^{3}: b^{3}\right)\right\}\right)$ (which describes the projection $\psi_{2}$ from $(0: 0: 1: 0)$, with $\left.\alpha_{2}=\psi_{2}^{*}\left(x_{0}\right)\right)$.

- The Dynkin diagram is:

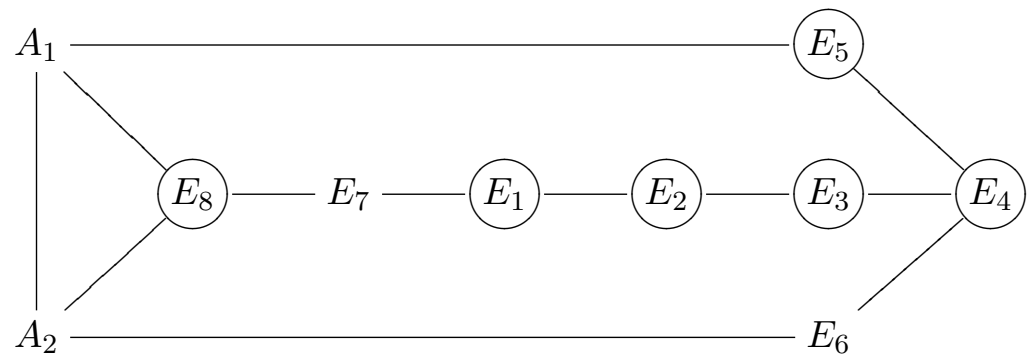

- The anticanonical embedding is given by

$\left(\phi^{*}\left(x_{i}\right)\right)=\left(\eta_{8} \alpha_{2}, \eta^{(2,2,2,2,1,1,2)} \eta_{8}, \eta^{(1,2,3,4,2,3,0)}, \eta^{(1,1,1,1,1,0,1)} \eta_{8} \alpha_{1}\right)$.

Type xx $\left(\mathbf{E}_{6}\right)$. Type $x x$ has been considered in [HT04, Section 3]. Its properties are: 
- The equation is

$$
x_{1} x_{2}^{2}+x_{2} x_{0}^{2}+x_{3}^{3}=0 .
$$

- The singularity $(0: 1: 0: 0)$ gives exceptional divisors $E_{1}, \ldots, E_{6}$, and there is a unique line $E_{7}=\left\{x_{2}=x_{3}=0\right\}$.

- A basis of $\operatorname{Pic}(\widetilde{S})$ is $E_{1}, \ldots, E_{7}$, with $-K_{\widetilde{S}}=(2,3,4,4,5,6,3)$.

- The Cox ring is

$\operatorname{Cox}(\widetilde{S})=\mathbb{K}\left[\eta_{1}, \ldots, \eta_{7}, \alpha_{1}, \alpha_{2}, \alpha_{3}\right] /\left(\eta_{4}^{2} \eta_{5} \eta_{7}^{3} \alpha_{3}+\eta_{2} \alpha_{2}^{2}+\eta_{1}^{2} \eta_{3} \alpha_{1}^{3}\right)$, where the relation is of degree $(2,3,4,6,6,6,6)$, and $\operatorname{deg}\left(\alpha_{1}\right)=$ $A_{1}=(0,1,1,2,2,2,2)=\psi^{*}\left(\left\{\left(a b: a^{2}:-b^{2}: 0\right)\right\}\right)$ describes a projection $\psi_{1}$ from $E_{7}$ with $\alpha_{1}=\psi_{1}^{*}\left(x_{3}\right)$, and $\operatorname{deg}\left(\alpha_{2}\right)=A_{2}=$ $(1,1,2,3,3,3,3)=\psi^{*}\left(\left\{\left(0:-a^{3}: b^{3}: a b^{2}\right)\right\}\right)$ describes the projection $\psi_{2}$ from the singularity with $\alpha_{2}=\psi_{2}^{*}\left(x_{0}\right)$, and $\operatorname{deg}\left(\alpha_{3}\right)=A_{3}=$ $(2,3,4,4,5,6,3)=-K_{\widetilde{S}}=\psi^{*}\left(\left\{\left(a^{3}: 0:-b^{3}: a^{2} b\right)\right\}\right)$ describes the anticanonical embedding $\phi$ with $\alpha_{3}=\phi^{*}\left(x_{1}\right)$.

- The extended Dynkin diagram is:

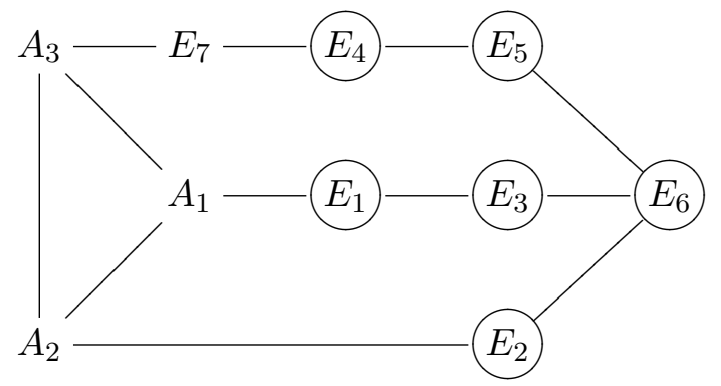

Here, $A_{1}, A_{2}, A_{3}$ meet in $(0: 0: 1: 0)$.

- The anticanonical embedding $\phi$ is given by

$$
\left(\phi^{*}\left(x_{i}\right)\right)=\left(\eta^{(1,2,2,1,2,3,0)} \alpha_{2}, \alpha_{3}, \eta^{(2,3,4,4,5,6,3)}, \eta^{(2,2,3,2,3,4,1)} \alpha_{1}\right) .
$$





\section{CHAPTER 6}

\section{Cox rings of generalized Del Pezzo surfaces}

\subsection{Introduction}

Let $\widetilde{S}_{r}$ be the blow-up of $\mathbb{P}^{2}$ in $r \leq 8$ points in almost general position over an algebraically closed field $\mathbb{K}$, i.e., a generalized Del Pezzo surface of degree $9-r$ (Section 1.5). We restrict ourselves to the case $r \leq 7$.

If $\widetilde{S}_{r}$ is a smooth Del Pezzo surface, the Cox ring is known explicitly (see Chapter 3). For generalized Del Pezzo surfaces, the Cox ring can be determined for toric $\widetilde{S}_{r}$ by results of Cox (see Lemma 2.12), and in cases with exactly one relation in the Cox ring by a method of Hassett and Tschinkel (see Chapter 5).

The purpose of this chapter is to show how generators and relations (up to radical) of $\operatorname{Cox}\left(\widetilde{S}_{r}\right)$ can be determined explicitly for any generalized Del Pezzo surface, in particular including Cox rings with more than one relation.

Compared to the case of non-toric smooth Del Pezzo surfaces, this problem has several complications:

- While there is only one type of smooth Del Pezzo surfaces in each degree, there are, for example, 20 more types of generalized cubic Del Pezzo surface.

- In the smooth case, every generator of the Cox ring corresponds to a negative curve. For generalized Del Pezzo surfaces, extra generators can occur (see Chapter 5 for examples).

- The Weyl group symmetry of the configuration of $(-1)$-curves on smooth Del Pezzo surfaces (Section 1.4) can be used when determining the Cox ring. The configuration of negative curves (including (-2)-curves) on generalized Del Pezzo surfaces is usually less symmetric.

However, we will refine arguments of BP04 in Section 6.2 to describe generators of $\operatorname{Cox}\left(\widetilde{S}_{r}\right)$. Every generator corresponds to a negative curve on $\widetilde{S}_{r}$, or has degree $-K_{\widetilde{S}_{r}}$, or is the pull-back of a generator of $\operatorname{Cox}\left(\widetilde{S}_{r-1}\right)$, where $\widetilde{S}_{r-1}$ is obtained by the contraction of a $(-1)$-curve on $\widetilde{S}_{r}$.

Having determined $N_{r}$ generators of $\operatorname{Cox}\left(\widetilde{S}_{r}\right)$, we may think of $\operatorname{Cox}\left(\widetilde{S}_{r}\right)$ in terms of the affine variety $\mathbb{A}\left(\widetilde{S}_{r}\right)=\operatorname{Spec}\left(\operatorname{Cox}\left(\widetilde{S}_{r}\right)\right)$ embedded in $\mathbb{A}^{N_{r}}$. Determining the ideal of relations in $\operatorname{Cox}\left(\widetilde{S}_{r}\right)$ up to radical is equivalent to finding equations defining $\mathbb{A}\left(\widetilde{S}_{r}\right)$.

The ideas of [HT04] help to find certain relations in $\operatorname{Cox}\left(\widetilde{S}_{r}\right)$ even when there is more than one relation. However, the proof of [HT04 that the single relation defines $\operatorname{Cox}\left(\widetilde{S}_{r}\right)$ does not generalize to a situation where more relations occur. 
Instead, we proceed as follows. Let $E$ be a $(-1)$-curve on $\widetilde{S}_{r}$. Let $U_{E}$ be the open subset of $\mathbb{A}^{N}$ where the coordinate corresponding to $E$ does not vanish. By Lemma 1.35, contracting $E$ results in a generalized Del Pezzo surface $\widetilde{S}_{r-1}$. We will see that

$$
\mathbb{A}\left(\widetilde{S}_{r}\right) \cap U_{E} \cong \mathbb{A}\left(\widetilde{S}_{r-1}\right) \times\left(\mathbb{A}^{1} \backslash\{0\}\right) .
$$

This will allow us to determine $\mathbb{A}\left(\widetilde{S}_{r}\right) \cap U_{E}$ recursively, and since $\mathbb{A}\left(\widetilde{S}_{r}\right)$ is irreducible, we obtain the ideal of relations up to radical by taking the closure. See Section 6.3 for more details.

In a series of examples of quartic and cubic Del Pezzo surfaces, we will see that the methods to determine the generators and relations in $\operatorname{Cox}\left(\widetilde{S}_{r}\right)$, as described in Sections 6.2 and 6.3, can be made explicit in practice. As our approach relates $\operatorname{Cox}\left(\widetilde{S}_{r}\right)$ to the case $r-1$, we apply the results for degree 5 (where the Cox ring of every singular surface has at most one relation) of Section 5.4 and degree 4 of Section 5.5 whenever the surface $\widetilde{S}_{r-1}$ obtained from $\widetilde{S}_{r}$ by contracting a $(-1)$-curve has a Cox ring with exactly one relation.

In the examples, we use notation from previous chapters, in particular Chapter 5.

\subsection{Generators}

In this section, we show how to determine generators of $\operatorname{Cox}\left(\widetilde{S}_{r}\right)$, where $\widetilde{S}_{r}$ is the minimal desingularization of a singular Del Pezzo surface $S_{r}$ of degree $9-r \geq 2$.

Lemma 6.1. For $2 \leq r \leq 7$, let $\widetilde{S}_{r}$ be a generalized Del Pezzo surface of degree $9-r$. Let $D \in \operatorname{Pic}\left(\widetilde{\widetilde{S}}_{r}\right)$ such that $(D, E) \geq 0$ for all negative curves $E$ on $\widetilde{S}_{r}$. Then the linear system $|D|$ has no base points.

Proof. We use induction on $r$, starting with the case $r=2$. The surface $\widetilde{S}_{2}$ of degree 7 can be a smooth Del Pezzo surface or a generalized Del Pezzo surface of type $\mathbf{A}_{1}$ which is obtained from $\mathbb{P}^{2}$ by blowing up one point and another point on the exceptional divisor of the first blow-up. The smooth case is covered by BP04, Proposition 2.3]. In the second case, we have (in terms of the standard basis as in Lemma 1.7) the $(-2)$-curve $E_{1}=l_{1}-l_{2}$ and the $(-1)$-curves $E_{2}=l_{2}$ and $E_{3}=l_{0}-l_{1}-l_{2}$. The negative curves $E_{1}, E_{2}, E_{3}$ are also a basis of $\operatorname{Pic}\left(\widetilde{S}_{2}\right)$. The dual basis with respect to the intersection form consists of $l_{0}-l_{1}$ (defining a conic bundle fibration $\widetilde{S}_{2} \rightarrow \mathbb{P}^{1}$ ), $2 l_{0}-l_{1}-l_{2}$ (defining a morphism $\widetilde{S}_{2} \rightarrow \mathbb{P}^{3}$ ), and $l_{0}$ (defining $\widetilde{S}_{2} \rightarrow \mathbb{P}^{2}$ ), so the corresponding linear systems have no base points. A general $D$ is a non-negative integral linear combination of these three elements, and, therefore, $|D|$ also has no base points.

For $r \geq 3$, if there is a $(-1)$-curve $E \subset \widetilde{S}_{r}$ with $(D, E)=0$, then we consider the map $\pi_{E}: \widetilde{S}_{r} \rightarrow \widetilde{S}_{r-1}$ which contracts $E$, where $\widetilde{S}_{r-1}$ is a generalized Del Pezzo surface of degree $9-(r-1)$. We have $\mathcal{O}(D)=\pi_{E}^{*} \mathcal{O}\left(D^{\prime}\right)$ for some invertible sheaf $\mathcal{O}\left(D^{\prime}\right)$ on $\widetilde{S}_{r-1}$. As the negative curves of $\widetilde{S}_{r-1}$ are images of negative curves of $\widetilde{S}_{r}$ under $\pi_{E}$, the divisor $D^{\prime}$ is nef. By induction on $r$, the linear system $\left|D^{\prime}\right|$ has no base points. Consequently, $|D|=\left|\pi_{E}^{*} D^{\prime}\right|$ has no base points. 
If there is no $(-1)$-curve $E \subset \widetilde{S}_{r}$ with $(D, E)=0$, then let $m>0$ be the minimal number $(D, E)$ for all $(-1)$-curves $E$. Since $\left(E,-K_{\widetilde{S}_{r}}\right)=1$ for all $(-1)$-curves and $\left(E,-K_{\widetilde{S}_{r}}\right)=0$ for all $(-2)$-curves, $D^{\prime}:=D-m \cdot\left(-K_{\widetilde{S}_{r}}\right)$ is nef, and $\left(D^{\prime}, E\right)=0$ for some $(-1)$-curve $E$. As discussed before, $\left|D^{\prime}\right|$ has no base points. Since $r \leq 7$, the system $\left|-K_{\widetilde{S}_{r}}\right|$ is also base point free. Therefore, $|D|$ has no base points.

For $r=2$, the Del Pezzo surfaces $S_{2}$ of degree 7 are toric. Hence, generators of $\operatorname{Cox}\left(\widetilde{S}_{2}\right)$ are known (Lemma 2.12). For larger $r$, they can be determined recursively as follows. Suppose that we can find a finite set of generators of $\operatorname{Cox}\left(\widetilde{S}_{r-1}\right)$ for any generalized Del Pezzo surface of degree $9-(r-1)$.

For every (-1)-curve $E$ on $\widetilde{S}_{r}$, let $\pi_{E}: \widetilde{S}_{r} \rightarrow \widetilde{S}_{r-1}$ be the contraction of $E$ resulting in a generalized Del Pezzo surface $\widetilde{S}_{r-1}$, and choose a homogeneous system of generators of $\operatorname{Cox}\left(\widetilde{S}_{r-1}\right)$.

An element of $\operatorname{Cox}\left(\widetilde{S}_{r}\right)$ is called a distinguished section if it is one of the following:

- A section $\xi(E)$ corresponding to a negative curve $E$ on $\widetilde{S}_{r}$ (cf. Lemma 1.3 .

- A global section of $-K_{\widetilde{S}_{r}}$.

- A section $\pi_{E}^{*}(\alpha)$, where $\pi_{E}: \widetilde{S}_{r} \rightarrow \widetilde{S}_{r-1}$ is as above, and the section $\alpha$ is an element of the chosen homogeneous system of generators of $\operatorname{Cox}\left(\widetilde{S}_{r-1}\right)$ which does not vanish in $\pi_{E}(E)$ and does not correspond to a negative curve on $\widetilde{S}_{r-1}$.

Theorem 6.2. For $3 \leq r \leq 7$, the Cox ring $\operatorname{Cox}\left(\widetilde{S}_{r}\right)$ is generated by a finite set of distinguished sections which can be determined explicitly, including at most two global sections of $-K_{\widetilde{S}_{r}}$.

Proof. Let $k(D)$ be the number of fixed components of $D$ which are $(-2)$-curves of $\widetilde{S}_{r}$. If $k(D)>0$ and the $(-2)$-curve $E$ is a fixed component of $D$, then $k(D-E)=k(D)-1$. If $k(D)=0$, then $(D, E) \geq 0$ for any $(-2)$-curve $E$ since $E$ is the only prime divisor which intersects $E$ negatively.

For each effective divisor $D$ on $\widetilde{S}_{r}$, we want to show that $H^{0}\left(\widetilde{S}_{r}, \mathcal{O}(D)\right)$ is generated by the distinguished sections. We use induction over $\operatorname{deg}(D):=$ $\left(D,-K_{\widetilde{S}_{r}}\right)$. Note that $D$ is not effective if $\operatorname{deg}(D)<0$. In each degree, we use a second induction over $k(D)$.

(1) If there exists a negative curve $E$ with $(D, E)<0$, then $E$ is a fixed component of $D$. The sequence

$$
H^{0}\left(\widetilde{S}_{r}, \mathcal{O}(D-E)\right) \rightarrow H^{0}\left(\widetilde{S}_{r}, \mathcal{O}(D)\right) \rightarrow H^{0}\left(E,\left.\mathcal{O}(D)\right|_{E}\right)
$$

is exact, where the first map is multiplication with $\xi(E)$. Since $H^{0}\left(E,\left.\mathcal{O}(D)\right|_{E}\right)=0$, the claim follows from the induction assumption for $D-E$, where $k(D-E)<k(D)$ if $E$ is a (-2)-curve, and $\operatorname{deg}(D-E)<\operatorname{deg}(D)$ if $E$ is a $(-1)$-curve.

(2) If there exists a $(-1)$-curve $E$ with $(D, E)=0$, consider the map $\pi_{E}: \widetilde{S}_{r} \rightarrow \widetilde{S}_{r-1}$ contracting $E$. Then $\mathcal{O}(D) \cong \pi_{E}^{*} \mathcal{O}\left(D^{\prime}\right)$, and

$$
\pi_{E}^{*}: H^{0}\left(\widetilde{S}_{r-1}, \mathcal{O}\left(D^{\prime}\right)\right) \rightarrow H^{0}\left(\widetilde{S}_{r}, \mathcal{O}(D)\right)
$$


is an isomorphism. We must show that, for any element $\alpha$ of the chosen set of generators of $\operatorname{Cox}\left(\widetilde{S}_{r-1}\right)$, the section $\pi_{E}^{*}(\alpha)$ is a combination of the distinguished sections of $\widetilde{S}_{r}$.

Indeed, let $A$ be the divisor corresponding to $\alpha$, let $j$ be the multiplicity of $\pi_{E}(E)$ on $A$, and let $\widehat{A}$ be the strict transform of $A$, with corresponding section $\widehat{\alpha}$. Then $\pi_{E}^{*}(A)=\widehat{A}+j E$, and

$$
\pi_{E}^{*}(\alpha)=c \cdot \xi(E) \cdot \widehat{\alpha} \quad \text { for some } c \in \mathbb{K}^{*} .
$$

If $j>0$, then $\operatorname{deg}(\widehat{A})=\operatorname{deg}(A)-j<\operatorname{deg}(A)$, so $\widehat{\alpha}$ is a combination of the distinguished sections by induction over the degree. If $j=0$ and $A$ is a negative curve on $\widetilde{S}_{r-1}$, then $\pi_{E}^{*}(A)$ is a negative curve on $\widetilde{S}_{r}$, so $\pi_{E}^{*}(\alpha)$ is a distinguished section. Otherwise, $\pi_{E}^{*}(\alpha)$ is a distinguished section by definition.

(3) If $D=-K_{\widetilde{S}_{r}}$ (or equivalently, $(D, E)=1$ for all $(-1)$-curves $E$ and $\left(D, E^{\prime}\right)=0$ for all $(-2)$-curves $\left.E^{\prime}\right)$, then $\left.\mathcal{O}(D)\right|_{E}$ is isomorphic to $\mathcal{O}_{E}(1)$ for any $(-1)$-curve $E$. We have the exact sequence

$0 \rightarrow H^{0}\left(\widetilde{S}_{r}, \mathcal{O}(D-E)\right) \rightarrow H^{0}\left(\widetilde{S}_{r}, \mathcal{O}(D)\right) \rightarrow H^{0}\left(E,\left.\mathcal{O}(D)\right|_{E}\right)$.

Since $\operatorname{deg}(D-E)<\operatorname{deg}(D), H^{0}\left(\widetilde{S}_{r}, \mathcal{O}(D-E)\right)$ is generated by distinguished sections by induction over the degree. Furthermore, $\operatorname{dim} H^{0}\left(E,\left.\mathcal{O}(D)\right|_{E}\right)=2$. Therefore, we must include at most two sections of $H^{0}\left(\widetilde{S}_{r}, \mathcal{O}(D)\right)$ in the set of distinguished sections.

(4) If $(D, E) \geq 1$ for all $(-1)$-curves $E$ and $\left(D, E^{\prime}\right) \geq 0$ for all $(-2)$ curves $E^{\prime}$, but $D \neq-K_{\widetilde{S}_{r}}$, let $m$ be the minimum of $(D, E)$ for all $(-1)$-curves $E$. Let $E_{0}$ be a $(-1)$-curve with $\left(D, E_{0}\right)=m$. Let $D^{\prime \prime}:=D-m \cdot\left(-K_{\widetilde{S}_{r}}\right)$. Consider the sequence

$0 \rightarrow H^{0}\left(\widetilde{S}_{r}, \mathcal{O}\left(D-E_{0}\right)\right) \rightarrow H^{0}\left(\widetilde{S}_{r}, \mathcal{O}(D)\right) \rightarrow H^{0}\left(E_{0},\left.\mathcal{O}(D)\right|_{E_{0}}\right)$,

where the first map is multiplication by the distinguished section $\xi\left(E_{0}\right)$. By induction over the degree, $H^{0}\left(\widetilde{S}_{r}, \mathcal{O}\left(D-E_{0}\right)\right)$ is generated by distinguished sections. Therefore, it is enough show that $H^{0}\left(\widetilde{S}_{r}, \mathcal{O}(D)\right)$ contains elements generated by distinguished sections that map to generators of $H^{0}\left(E_{0},\left.\mathcal{O}(D)\right|_{E_{0}}\right)$. Since $\left(D^{\prime \prime}, E_{0}\right)=$ 0 , the space $H^{0}\left(\widetilde{S}_{r}, \mathcal{O}\left(D^{\prime \prime}\right)\right)$ is generated by distinguished sections by case (2) and contains a section $s$ which can be chosen such that its support does not contain $E_{0}$ by Lemma 6.1. Multiplication by $s$ gives a homomorphism

$$
H^{0}\left(\widetilde{S}, \mathcal{O}\left(m \cdot\left(-K_{\widetilde{S}_{r}}\right)\right) \rightarrow H^{0}\left(\widetilde{S}_{r}, \mathcal{O}(D)\right)\right.
$$

which restricts to an isomorphism

$$
H^{0}\left(E_{0},\left.\mathcal{O}\left(m \cdot\left(-K_{\widetilde{S}_{r}}\right)\right)\right|_{E_{0}}\right) \cong H^{0}\left(E_{0},\left.\mathcal{O}(D)\right|_{E_{0}}\right)
$$

since $\left(D^{\prime \prime}, E_{0}\right)=0$ implies that $s$ is non-zero on $E_{0}$. Therefore, it remains to show that

$$
H^{0}\left(E_{0},\left.\mathcal{O}\left(m \cdot\left(-K_{\widetilde{S}_{r}}\right)\right)\right|_{E_{0}}\right) \cong H^{0}\left(E_{0}, \mathcal{O}_{E_{0}}(m)\right)
$$

can be obtained by the restriction of elements of $H^{0}\left(\widetilde{S}_{r}, \mathcal{O}(m\right.$. $\left.\left.\left(-K_{\widetilde{S}_{r}}\right)\right)\right)$ that are generated by distinguished sections. For $m=1$, 
this has been proved in case (3). For $m>1$, this follows from the fact that $H^{0}\left(E_{0}, \mathcal{O}_{E_{0}}(m)\right)$ is generated by products of $m$ elements of $H^{0}\left(E_{0}, \mathcal{O}_{E_{0}}(1)\right)$.

As the number of negative curves on $\widetilde{S}_{r}$ is finite, the finiteness of the set of distinguished sections follows by induction on $r$, where for $r=2$, the toric surfaces $\widetilde{S}_{2}$ have a finite set of generators of $\operatorname{Cox}\left(\widetilde{S}_{2}\right)$ by Lemma 2.12 .

REMARK 6.3. Generators of $\operatorname{Cox}\left(\widetilde{S}_{r}\right)$ which do not correspond to negative curves on $\widetilde{S}_{r}$ will be called extra generators. As the following observations show, we do not expect to obtain many extra generators as $\pi_{E}^{*}(\alpha)$ via $\pi_{E}: \widetilde{S}_{r} \rightarrow \widetilde{S}_{r-1}$ from generators of $\operatorname{Cox}\left(\widetilde{S}_{r-1}\right)$.

- As the self intersection number decreases when a point is blown up (Lemma 1.2), extra generators of $\operatorname{Cox}\left(\widetilde{S}_{r}\right)$ can correspond only to extra generators of $\operatorname{Cox}\left(\widetilde{S}_{r-1}\right)$.

- Two contractions $\pi_{E}: \widetilde{S}_{r} \rightarrow \widetilde{S}_{r-1}$ and $\pi_{E^{\prime}}: \widetilde{S}_{r} \rightarrow \widetilde{S}_{r-1}^{\prime}$ of $(-1)$ curves $E$ and $E^{\prime}$ can lead to extra generators $\pi_{E}^{*}(\alpha)$ and $\pi_{E^{\prime}}^{*}\left(\alpha^{\prime}\right)$. However, one of them or both might be unnecessary for a minimal system of generators.

EXAMPLE 6.4. Starting with a surface $\widetilde{S}_{2}$ of type $\mathbf{A}_{1}$, four blow-ups lead to a cubic surface $\widetilde{S}_{6}$ of type $\mathbf{E}_{6}$ :

\begin{tabular}{|c||c|c|c|c|c|}
\hline$r$ & 2 & 3 & 4 & 5 & 6 \\
\hline \hline$S_{r}$ & $\mathbf{A}_{1}$ & $\mathbf{A}_{1}+\mathbf{A}_{2}$ & $\mathbf{A}_{4}$ & $\mathbf{D}_{5}$ & $\mathbf{E}_{6}$ \\
\hline
\end{tabular}

Generators of the Cox rings of each $\widetilde{S}_{r}$ were already determined in Chapter 5 (we also use notation from that chapter), but we show how they are interpreted in view of Theorem 6.2.

- The surface $\widetilde{S}_{2}$ of type $\mathbf{A}_{1}$ has a Cox ring which is generated by sections of the divisors $\eta_{1}, \eta_{2}, \eta_{3}, \alpha_{1}, \alpha_{2}$ corresponding to the cyclic Dynkin diagram $(-2,-1,-1,1,0)$ of $\widetilde{S}_{2}$ (Table 1.4).

- The surface $\widetilde{S}_{3}$ is obtained from $\widetilde{S}_{2}$ by blowing up the intersection of the $(-1)$-curves, giving a toric variety with cyclic Dynkin diagram $(-2,-2,-1,-2,1,0)$. By Theorem 6.2, the distinguished sections which generate $\operatorname{Cox}\left(\widetilde{S}_{3}\right)$ are sections $\eta_{1}, \ldots, \eta_{4}$ of the four negative curves and the transforms of the sections $\alpha_{1}, \alpha_{2}$ of the (1)- and (0)-divisor of $\widetilde{S}_{2}$ which we also call $\alpha_{1}, \alpha_{2}$ for simplicity. We check that we do not need to add further sections of $H^{0}\left(\widetilde{S}_{3},-K_{\widetilde{S}_{3}}\right)$.

- The surface $\widetilde{S}_{4}$ is obtained from $\widetilde{S}_{3}$ by blowing up a point on the (-1)-curve. This gives generators $\eta_{1}, \ldots, \eta_{5}$ corresponding to the negative curves, and transforms of $\alpha_{1}, \alpha_{2}$ of $\widetilde{S}_{3}$. By case $(3)$ of the proof, we must consider the restriction of $H^{0}\left(\widetilde{S}_{4},-K_{\widetilde{S}_{4}}\right)$ to the $(-1)$-curve $E_{5}$ : The generators $\eta_{i}, \alpha_{i}$ induce the sections

$\left(\eta^{(2,4,6,3,5)}, \eta^{(2,3,4,2,3)} \alpha_{1}, \eta^{(2,2,2,1,1)} \alpha_{1}^{2}, \eta^{(1,1,1,1,0)} \alpha_{1} \alpha_{2}, \eta^{(1,2,3,2,2)} \alpha_{2}\right)$.

Among these, only $\eta^{(1,1,1,1,0)} \alpha_{1} \alpha_{2}$ is non-zero after restriction to $E_{5}$. Hence, a section $\alpha_{3} \in H^{0}\left(\widetilde{S}_{4},-K_{\widetilde{S}_{4}}\right)$ completes the generating set. 
- Blowing up the intersection of the $(-1)$-curve of $\widetilde{S}_{4}$ and the divisor corresponding to $\alpha_{3}$ gives $\widetilde{S}_{5}$. The distinguished sections include $\eta_{1}, \ldots, \eta_{6}$ corresponding to the negative curves and $\alpha_{1}, \alpha_{2}$ as transforms of the generators in the previous case. We do not need the transform of $\alpha_{3}$ since $\alpha_{3}$ vanishes in the blown-up point. Only $\eta^{(2,1,1,2,2,0)} \alpha_{2}^{2}$ restricts to a non-zero element of $H^{0}\left(E_{6},-\left.K_{\widetilde{S}_{6}}\right|_{E_{6}}\right)$, so we need to add another section of $-K_{\widetilde{S}_{6}}$ which we call $\alpha_{3}$ again.

- Blowing up the intersection of the (-1)-curve and the divisor corresponding to $\alpha_{3}$ on $\widetilde{S}_{5}$ gives $\widetilde{S}_{6}$. As before, the distinguished sections are $\eta_{1}, \ldots, \eta_{7}$ corresponding to the negative curves, $\alpha_{1}, \alpha_{2}$ as transforms of the extra generators of $\operatorname{Cox}\left(\widetilde{S}_{5}\right)$ which do not vanish in the blown-up point, and a section $\alpha_{3}$ of $-K_{\widetilde{S}_{6}}$.

\subsection{Relations}

Let $\widetilde{S}_{r}$ be a generalized Del Pezzo surface of degree $9-r$. In this section, we show how to determine the relations in $\operatorname{Cox}\left(\widetilde{S}_{r}\right)$ up to radical.

For $r \leq 2, \widetilde{S}_{r}$ is toric, so there are no relations in $\operatorname{Cox}\left(\widetilde{S}_{r}\right)$. For $r \geq 3$, let $\pi_{E}: \widetilde{S}_{r} \rightarrow \widetilde{S}_{r-1}$ be the contraction of a $(-1)$-curve $E$ on $\widetilde{S}_{r}$, where $\widetilde{\widetilde{S}}_{r-1}$ is a generalized Del Pezzo surface of degree $9-(r-1)$. Let $\mathbb{A}\left(\widetilde{S}_{r}\right)$ be the affine variety $\operatorname{Spec}\left(\operatorname{Cox}\left(\widetilde{S}_{r}\right)\right)$.

Lemma 6.5. Let $U_{E}$ be the open subset $\{\xi(E) \neq 0\}$ of $\mathbb{A}\left(\widetilde{S}_{r}\right)$. Then

$$
\mathbb{A}\left(\widetilde{S}_{r}\right) \cap U_{E} \cong \mathbb{A}\left(\widetilde{S}_{r-1}\right) \times\left(\mathbb{A}^{1} \backslash\{0\}\right) .
$$

Proof. Using the fact $\operatorname{Pic}\left(\widetilde{S}_{r}\right)=\pi_{E}^{*}\left(\operatorname{Pic}\left(\widetilde{S}_{r-1}\right)\right) \oplus \mathbb{Z} \cdot E$, the proof is the same as the proof of [BP04, Proposition 4.4].

Having found generators $\xi_{1}, \ldots, \xi_{N_{r}}$ of $\operatorname{Cox}\left(\widetilde{S}_{r}\right)$, we are interested in the ideal defining $\mathbb{A}\left(\widetilde{S}_{r}\right)$ in $\mathbb{A}^{N_{r}}=$ Spec $R_{r}$, where $R_{r}=\mathbb{K}\left[\xi_{1}, \ldots, \xi_{N_{r}}\right]$ is a free polynomial ring.

Suppose that we have found certain relations between $\xi_{1}, \ldots, \xi_{N_{r}}$ which generate an ideal $J_{r}$. Let $Z_{r}:=\operatorname{Spec}\left(R_{r} / \operatorname{rad}\left(J_{r}\right)\right) \subset \mathbb{A}^{N_{r}}$. We want to check whether we have found all relations: While $\mathbb{A}\left(\widetilde{S}_{r}\right) \subset Z_{r}$ is clear, we need to check whether $\mathbb{A}\left(\widetilde{S}_{r}\right)=Z_{r}$. This can be done by the following method:

- Suppose that we have determined $J_{r-1}$ such that

$$
\mathbb{A}\left(\widetilde{S}_{r-1}\right) \cong \operatorname{Spec}\left(R_{r-1} / \operatorname{rad}\left(J_{r-1}\right)\right),
$$

where $\widetilde{S}_{r-1}$ is the generalized Del Pezzo surface obtained by contraction of the (-1)-curve $E$ on $\widetilde{S}_{r}$. First, we check that $Z_{r} \cap U_{E} \cong$ $\mathbb{A}\left(\widetilde{S}_{r-1}\right) \times\left(\mathbb{A}^{1} \backslash\{0\}\right)$. For this, we must show that that $\xi(E)$ and the generators of $\operatorname{Cox}\left(\widetilde{S}_{r}\right)$ which correspond to generators of $\operatorname{Cox}\left(\widetilde{S}_{r-1}\right)$, together with the relations in $J_{r}$, determine all other generators of $\operatorname{Cox}\left(\widetilde{S}_{r}\right)$. Furthermore, we must show that $J_{r}$ induces the relations in $\operatorname{Cox}\left(\widetilde{S}_{r-1}\right)$. Together with Lemma 6.5, this will prove

$$
Z_{r} \cap U_{E} \cong \mathbb{A}\left(\widetilde{S}_{r}\right) \cap U_{E} .
$$


- As $\mathbb{A}\left(\widetilde{S}_{r}\right)$ is irreducible by Lemma 2.9 and closed in $\mathbb{A}^{N_{r}}$, we can calculate the closure of $Z_{r} \cap U_{E}$ in $\mathbb{A}^{N_{r}}$. If this coincides with $Z_{r}$, then we are done, since in this case

$$
Z_{r}=\overline{Z_{r} \cap U_{E}}=\overline{\mathbb{A}\left(\widetilde{S}_{r}\right) \cap U_{E}}=\mathbb{A}\left(\widetilde{S}_{r}\right) .
$$

- Carrying out the first step of checking $Z_{r} \cap U_{E}=\mathbb{A}\left(\widetilde{S}_{r}\right) \cap U_{E}$ for all $(-1)$-curves $E$ instead of just one, the second step of show$\operatorname{ing} Z_{r}=\overline{Z_{r} \cap U}$ can be done with $U=U_{E}$ replaced by $U=$ $\bigcup_{(-1) \text {-curves } E} U_{E}$. In practice, this can be considerably simpler.

We illustrate this in a quartic and a cubic case. The types are numbered as in Tables 5.4 and 6.2 respectively Tables 5.5 and 6.3 .

Example 6.6 (Quartic type ii (2 $\mathbf{A}_{1}$ with nine lines)). We use Theorem 6.2 to show that the generators of $\operatorname{Cox}\left(\widetilde{S}_{5}\right)$ correspond to the negative curves $E_{1}, \ldots, E_{11}$ as in the following extended Dynkin diagram:

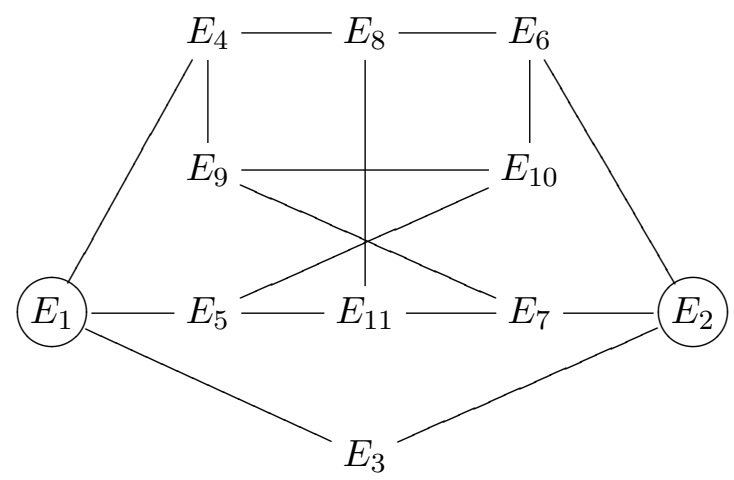

We claim that the relations are:

$$
\begin{aligned}
& R_{1}=\eta_{1} \eta_{2} \eta_{3}^{2}+\eta_{8} \eta_{11}+\eta_{9} \eta_{10} \\
& R_{2}=\eta_{1} \eta_{3} \eta_{4}+\eta_{6} \eta_{10}+\eta_{7} \eta_{11} \\
& R_{3}=\eta_{1} \eta_{3} \eta_{5}+\eta_{6} \eta_{8}+\eta_{7} \eta_{9} \\
& R_{4}=\eta_{2} \eta_{3} \eta_{6}+\eta_{4} \eta_{9}+\eta_{5} \eta_{11} \\
& R_{5}=\eta_{2} \eta_{3} \eta_{7}+\eta_{4} \eta_{8}+\eta_{5} \eta_{10}
\end{aligned}
$$

\begin{tabular}{|c|c|c|c|}
\hline$E_{i}$ & type of $S_{4}$ & other variables & generation relations \\
\hline \hline$E_{3}$ & $0:-$ & - & $R_{1}, R_{2}, R_{3}, R_{4}, R_{5}$ \\
$E_{4}$ & $i: \mathbf{A}_{1}$ & $\eta_{8}: R_{5}, \eta_{9}: R_{4}$ & $R_{2}$ \\
$E_{5}$ & $i: \mathbf{A}_{1}$ & $\eta_{10}: R_{5}, \eta_{11}: R_{4}$ & $R_{3}$ \\
$E_{6}$ & $i: \mathbf{A}_{1}$ & $\eta_{8}: R_{3}, \eta_{10}: R_{2}$ & $R_{4}$ \\
$E_{7}$ & $i: \mathbf{A}_{1}$ & $\eta_{9}: R_{3}, \eta_{11}: R_{2}$ & $R_{5}$ \\
$E_{8}$ & $i i: 2 \mathbf{A}_{1}$ & $\eta_{4}: R_{5}, \eta_{6}: R_{3}, \eta_{11}: R_{1}$ & - \\
$E_{9}$ & $i i: 2 \mathbf{A}_{1}$ & $\eta_{4}: R_{4}, \eta_{7}: R_{3}, \eta_{10}: R_{1}$ & - \\
$E_{10}$ & $i i: 2 \mathbf{A}_{1}$ & $\eta_{5}: R_{5}, \eta_{6}: R_{2}, \eta_{9}: R_{1}$ & - \\
$E_{11}$ & $i i: 2 \mathbf{A}_{1}$ & $\eta_{5}: R_{4}, \eta_{7}: R_{2}, \eta_{8}: R_{1}$ & - \\
\hline
\end{tabular}

TABLE 6.1. Quartic type $i i\left(2 \mathbf{A}_{1}\right)$ 
Depending on the choice of the $(-1)$-curve $E_{i}(i \in\{3, \ldots, 11\})$, we obtain a quintic Del Pezzo surface $S_{4}$ : In Table 6.1. we list the variables $\eta_{j}$ which do not correspond to negative curves on $S_{4}$, together with the relation $R_{k}$ which determines $\eta_{j}$, in the column "other variables". The column "generating relations" lists the relations which generate the ideal $J_{4}$ defining $\operatorname{Cox}\left(\widetilde{S}_{4}\right)$. The remaining relations $R_{k}$ turn out to be trivial or dependent on the generating relations in $\operatorname{Cox}\left(\widetilde{S}_{4}\right)$.

This shows that $Z_{5} \cap U=\mathbb{A}\left(\widetilde{S}_{5}\right) \cap U$, where $U$ is the complement of

$$
V=\left\{\left(\eta_{1}, \ldots, \eta_{11}\right) \in \mathbb{A}^{11} \mid \eta_{3}=\cdots=\eta_{11}=0\right\} .
$$

In particular, with

$$
U^{\prime}=\left\{\left(\eta_{1}, \ldots, \eta_{11}\right) \in \mathbb{A}^{11} \mid \eta_{11} \neq 0, \eta_{3}=\cdots=\eta_{10}=0\right\} \subset U,
$$

we have $\mathbb{A}\left(\widetilde{S}_{5}\right) \cap U^{\prime}=Z_{5} \cap U^{\prime}=U^{\prime}$. As $V \subset \overline{U^{\prime}}$, and $\mathbb{A}\left(\widetilde{S}_{5}\right)$ and $Z_{5}$ are closed, we have $\mathbb{A}\left(\widetilde{S}_{5}\right) \cap V=Z_{5} \cap V=V$. We conclude $\mathbb{A}\left(\widetilde{S}_{5}\right)=Z_{5}$.

Example 6.7 (Cayley's cubic surface - type vii $\left(4 \mathbf{A}_{1}\right)$ ). The four $(-2)$ curves are $E_{1}, \ldots, E_{4}$. Six (-1)-curves $E_{i, j}$ intersect $E_{i}$ and $E_{j}$. Three (-1)curves $E_{(i, j),(k, l)}$ intersect each other and $E_{i, j}$ and $E_{k, l}$, where $\{i, j, k, l\}=$ $\{1,2,3,4\}$. The corresponding generators of $\operatorname{Cox}\left(\widetilde{S}_{6}\right)$ are

$$
\eta_{i}, \quad \eta_{i, j}, \quad \eta_{(i, j),(k, l)}=-\eta_{(k, l),(i, j)} .
$$

Six relations have the form

$$
R_{i, j}=\eta_{i} \eta_{i, k} \eta_{i, l}+\eta_{j} \eta_{j, k} \eta_{j, l}-\eta_{k, l} \eta_{(i, j),(k, l)},
$$

and three have the form

$$
R_{(i, j),(k, l)}=\eta_{i, j}^{2} \eta_{i} \eta_{j}-\eta_{k, l}^{2} \eta_{k} \eta_{l}+\eta_{(i, k),(j, l)} \eta_{(i, l),(j, k)} .
$$

These nine relations define $Z_{6}$ and were already described in [HB03] and DT06, Example 14].

Contracting $E_{i, j}$ results in a quartic surface $S_{5}$ of type $i i\left(2 \mathbf{A}_{1}\right)$ as in the previous example. The variable $\eta_{(i, j),(k, l)}$, which does not correspond to a generator of $\operatorname{Cox}\left(\widetilde{S}_{5}\right)$, is determined on $U_{E_{i, j}}$ by $R_{k, l}$, while the five relations in $\operatorname{Cox}\left(\widetilde{S}_{5}\right)$ are given by $R_{(i, j),(k, l)}, R_{i, k}, R_{i, l}, R_{j, k}, R_{j, l}$.

Contracting $E_{(i, j),(k, l)}$ gives a quartic surface $S_{5}$ of type $i x\left(4 \mathbf{A}_{1}\right)$. The variables $\eta_{i, j}, \eta_{k, l}, \eta_{(i, k),(j, l)}, \eta_{(i, l),(j, k)}$, which do not correspond to generators of $\operatorname{Cox}\left(\widetilde{S}_{5}\right)$ are determined on $U_{E_{(i, j),(k, l)}}$ by $R_{i, j}, R_{k, l}, R_{(i, l),(j, k)}, R_{(i, k),(j, l)}$, respectively. As $S_{5}$ is toric, $\operatorname{Cox}\left(\widetilde{S}_{5}\right)$ has no relations. The other relations in $\operatorname{Cox}\left(\widetilde{S}_{6}\right)$ turn out trivial in $\operatorname{Cox}\left(\widetilde{S}_{5}\right)$.

Therefore, $Z_{6} \cap U=\mathbb{A}\left(\widetilde{S}_{6}\right) \cap U$, where $U$ is the complement of

$$
V=\left\{\eta_{i, j}=\eta_{(i, j),(k, l)}=0 \text { for all } i, j, k, l\right\} .
$$

In particular, we can check that $\mathbb{A}\left(\widetilde{S}_{6}\right) \cap U^{\prime}=Z_{6} \cap U^{\prime}=U^{\prime}$ where $U^{\prime} \subset U$ is defined as $V$ except that $\eta_{(1,2),(3,4)} \neq 0$. As $V \subset \overline{U^{\prime}}$, we have $\mathbb{A}\left(\widetilde{S}_{6}\right) \cap V=$ $Z_{6} \cap V=V$. Therefore, $\mathbb{A}\left(\widetilde{S}_{6}\right)=Z_{6}$. 
REMARK 6.8. In addition to the relations in $J_{r}$ that we identify in the first step of the process (corresponding to the relations in $J_{r-1}$ on the one hand, and determining the "other variables" on the other hand), we might need further relations in $J_{r}$ in order to describe $\mathbb{A}\left(\widetilde{S}_{r}\right)$ on the complement of $\bigcup_{(-1) \text {-curves } E} U_{E}$.

For example, this happens for the quartic type viii $\left(\mathbf{A}_{3}\right)$, as we will see in the following section.

\subsection{Degree 4}

\begin{tabular}{|c||c|c|c|c|c|}
\hline type & singularities & lines & extra gen. & gen. & relations \\
\hline \hline 0 & - & 16 & - & 16 & 20 \\
$i$ & $\mathbf{A}_{1}$ & 12 & - & 13 & 10 \\
$i i$ & $2 \mathbf{A}_{1}$ & 9 & - & 11 & 5 \\
$i i i$ & $2 \mathbf{A}_{1}$ & 8 & - & 10 & 2 \\
$i v$ & $\mathbf{A}_{2}$ & 8 & - & 10 & 2 \\
$v$ & $3 \mathbf{A}_{1}$ & 6 & - & 9 & 1 \\
vi & $\mathbf{A}_{2}+\mathbf{A}_{1}$ & 6 & - & 9 & 1 \\
vii & $\mathbf{A}_{3}$ & 5 & 1 & 9 & 1 \\
viii & $\mathbf{A}_{3}$ & 4 & 4 & 11 & 5 \\
ix & $4 \mathbf{A}_{1}$ & 4 & - & 8 & - \\
$x$ & $\mathbf{A}_{2}+2 \mathbf{A}_{1}$ & 4 & - & 8 & - \\
xi & $\mathbf{A}_{3}+\mathbf{A}_{1}$ & 3 & 2 & 9 & 1 \\
xii & $\mathbf{A}_{4}$ & 3 & 2 & 9 & 1 \\
xiii & $\mathbf{D}_{4}$ & 2 & 3 & 9 & 1 \\
xiv & $\mathbf{A}_{3}+2 \mathbf{A}_{1}$ & 2 & 1 & 8 & - \\
xv & $\mathbf{D}_{5}$ & 1 & 3 & 9 & 1 \\
\hline
\end{tabular}

TABle 6.2. Del Pezzo surfaces of degree 4

Complete results for generalized Del Pezzo surfaces whose degree is at least 5 can be found in Chapter 5. For smooth Del Pezzo surfaces, see BP04 and Chapter 3.

In degree 4, Section 5.5 lists all relevant information in the case of Del Pezzo surfaces whose universal torsor is an open subset of affine space or of an affine hypersurface, or equivalently, whose Cox ring has 8 or 9 generators. This includes exactly 10 of the 15 different types. We will describe generators and relations for the remaining five types here. We give similar information as in Chapter 5, using mostly the same notation and conventions.

For $\widetilde{S}_{5}$ of types $i$ to $i v$, we can check that $\operatorname{Cox}\left(\widetilde{S}_{5}\right)$ is generated by sections corresponding to negative curves. Their extended Dynkin diagrams can be found in CT88, Proposition 6.1].

\section{Type i $\left(\mathbf{A}_{1}\right)$.}

- The extended Dynkin diagram consists of one (-2)-curve $E_{0}$ and twelve (-1)-curves $E_{i}, E_{i, 1}, E_{i, 2}$ for $i \in\{1, \ldots, 4\}: E_{i}$ intersects $E_{0}$, $E_{i, 1}, E_{i, 2}$, while $E_{i, k}$ intersects $E_{i}$ and $E_{j, l}$ for $j \in\{1, \ldots, 4\} \backslash\{i\}$ and $l \neq k$. 
- A basis of $\operatorname{Pic}(\widetilde{S})$ is given by $E_{0}, \ldots, E_{4}, E_{1,1}$, with

$$
\begin{aligned}
E_{2,1}= & (0,1,-1,0,0,1), E_{3,1}=(0,1,0,-1,0,1), E_{4,1}=(0,1,0,0,-1,1), \\
E_{1,2}= & (1,-1,1,1,1,-1), E_{2,2}=(1,0,0,1,1,-1), E_{3,2}=(1,0,1,0,1,-1), \\
E_{4,2}= & (1,0,1,1,0,-1), \\
& \text { and }-K_{\widetilde{S}_{5}}=(2,1,1,1,1,0) .
\end{aligned}
$$

- For $i \neq j \in\{1, \ldots, 4\}$, there is one relation between

$$
\eta_{0} \eta_{i} \eta_{j}, \quad \eta_{k, 1} \eta_{l, 2}, \quad \eta_{l, 1} \eta_{k, 2}
$$

where $\{i, j, k, l\}=\{1, \ldots, 4\}$. For $i \in\{1,2\}$, there are two relations between

$$
\eta_{1} \eta_{1, i}, \quad \eta_{2} \eta_{2, i}, \quad \eta_{3} \eta_{3, i}, \quad \eta_{4} \eta_{4, i}
$$

This gives 10 relations in total.

- 23 monomial sections of $-K_{\widetilde{S}}$ are: $\eta_{0}^{2} \eta_{1} \eta_{2} \eta_{3} \eta_{4}$, six section of the form $\eta_{i, 1} \eta_{j, 1} \eta_{k, 2} \eta_{l, 2}$, twelve section of the form $\eta_{0} \eta_{i} \eta_{j} \eta_{i, 1} \eta_{j, 2}$, four section of the form $\eta_{0} \eta_{i}^{2} \eta_{i, 1} \eta_{i, 2}$

\section{Type ii ( $2 \mathrm{~A}_{1}$ with 9 lines).}

- See Example 6.6.

\section{Type iii (2A $\mathbf{A}_{1}$ with 8 lines).}

- Its equation is:

$$
x_{0} x_{1}-x_{2}^{2}=x_{0} x_{2}+x_{1} x_{2}+x_{3} x_{4}=0
$$

- Singularities: $(0: 0: 0: 1: 0)$ giving $E_{1}$, and $(0: 0: 0: 0: 1)$ giving $E_{2}$; Lines: $E_{3}=\left\{x_{0}=x_{2}=x_{3}=0\right\}, E_{4}=\left\{x_{0}-x_{1}=x_{0}+x_{2}=\right.$ $\left.x_{4}=0\right\}, E_{5}=\left\{x_{0}-x_{1}=x_{0}-x_{2}=x_{4}=0\right\}, E_{6}=\left\{x_{1}=x_{2}=x_{4}=\right.$ $0\}, E_{7}=\left\{x_{1}=x_{2}=x_{3}=0\right\}, E_{8}=\left\{x_{0}-x_{1}=x_{0}+x_{2}=x_{3}=0\right\}$, $E_{9}=\left\{x_{0}-x_{1}=x_{0}-x_{2}=x_{3}=0\right\}, E_{10}=\left\{x_{0}=x_{2}=x_{4}=0\right\}$.

- A basis of $\operatorname{Pic}(\widetilde{S})$ is given by $E_{1}, \ldots, E_{6}$, with

$$
\begin{aligned}
& E_{7}=(-1,1,-1,1,1,0), E_{8}=(-1,1,-1,1,0,1), \\
& E_{9}=(-1,1,-1,0,1,1), E_{10}=(-1,1,-2,1,1,1),
\end{aligned}
$$

and $-K_{\widetilde{S}_{5}}=(-1,3,-2,2,2,2)$.

- Dynkin diagram:

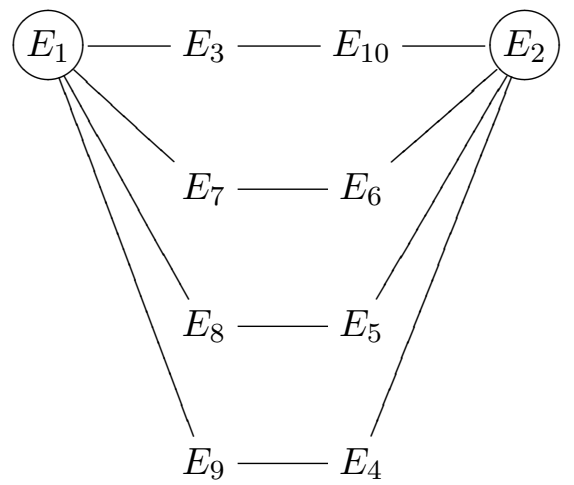


- The anticanonical map $\phi$ is given by

$$
\begin{aligned}
\left(\phi^{*}\left(x_{i}\right)\right)= & \left(\eta^{(1,1,2,0,0,0)} \eta_{10}^{2}, \eta^{(1,1,0,0,0,2)} \eta_{7}^{2}, \eta^{(1,1,1,0,0,1)} \eta_{7} \eta_{10}\right. \\
& \left.\eta^{(2,0,1,0,0,0)} \eta_{7} \eta_{8} \eta_{9}, \eta^{(0,2,0,1,1,1)} \eta_{10}\right),
\end{aligned}
$$

and furthermore, $\phi^{*}\left(x_{0}-x_{1}\right)=\eta^{(1,1,0,1,1,0)} \eta_{8} \eta_{9}, \phi^{*}\left(x_{0}-x_{2}\right)=$ $\eta^{(1,1,1,1,0,0)} \eta_{9} \eta_{10}, \phi^{*}\left(x_{0}+x_{2}\right)=\eta^{(1,1,1,0,1,0)} \eta_{8} \eta_{10}, \phi^{*}\left(x_{1}+x_{2}\right)=$ $\eta^{(1,1,0,0,1,1)} \eta_{7} \eta_{8}, \phi^{*}\left(x_{2}-x_{1}\right)=\eta^{(1,1,0,1,0,1)} \eta_{7} \eta_{9}, \phi^{*}\left(x_{0}+x_{1}+2 x_{2}\right)=$ $\eta^{(1,1,0,0,2,0)} \eta_{8}^{2}, \phi^{*}\left(x_{0}+x_{1}-2 x_{2}\right)=\eta^{(1,1,0,2,0,0)} \eta_{9}^{2}$.

- Let $Z_{5}$ be the affine variety defined by

$$
\begin{aligned}
& R_{1}=\eta_{3} \eta_{10}+\eta_{6} \eta_{7}-\eta_{5} \eta_{8} \\
& R_{2}=\eta_{3} \eta_{10}-\eta_{6} \eta_{7}-\eta_{4} \eta_{9}
\end{aligned}
$$

Contracting any line results in a quintic Del Pezzo surface of type $i\left(\mathbf{A}_{1}\right)$. We see easily that $\mathbb{A}\left(\widetilde{S}_{5}\right)=Z_{5}$ not only when restricted to the complement of $V=\left\{\eta_{3}=\cdots=\eta_{10}=0\right\}$, but also on $V$.

Type iv $\left(\mathbf{A}_{2}\right)$.

- Its equation is:

$$
x_{1} x_{2}-x_{3} x_{4}=x_{0}\left(x_{1}+x_{2}+x_{3}+x_{4}\right)-x_{3} x_{4}=0
$$

- Singularities: $(1: 0: 0: 0: 1)$ giving $E_{1}, E_{2}$; Lines: $E_{3}=\left\{x_{0}=\right.$ $\left.x_{1}=x_{3}=0\right\}, E_{4}=\left\{x_{0}=x_{2}=x_{4}=0\right\}, E_{5}=\left\{X=x_{2}=x_{3}=0\right\}$, $E_{6}=\left\{X=x_{1}=x_{4}=0\right\}, E_{7}=\left\{X=x_{2}=x_{4}=0\right\}, E_{8}=\{X=$ $\left.x_{1}=x_{3}=0\right\}, E_{9}=\left\{x_{0}=x_{1}=x_{4}=0\right\}, E_{10}=\left\{x_{0}=x_{2}=x_{3}=\right.$ $0\}$, where $X:=x_{1}+x_{2}+x_{3}+x_{4}$.

- A basis of $\operatorname{Pic}(\widetilde{S})$ is $E_{1}, \ldots, E_{6}$, with $E_{7}=(0,1,0,-1,1,1), E_{8}=$ $(0,1,-1,0,1,1), E_{9}=(1,2,-1,-1,2,1), E_{10}=(1,2,-1,-1,1,2)$, and $-K_{\widetilde{S}_{5}}=(2,4,-1,-1,3,3)$.

- Dynkin diagram:

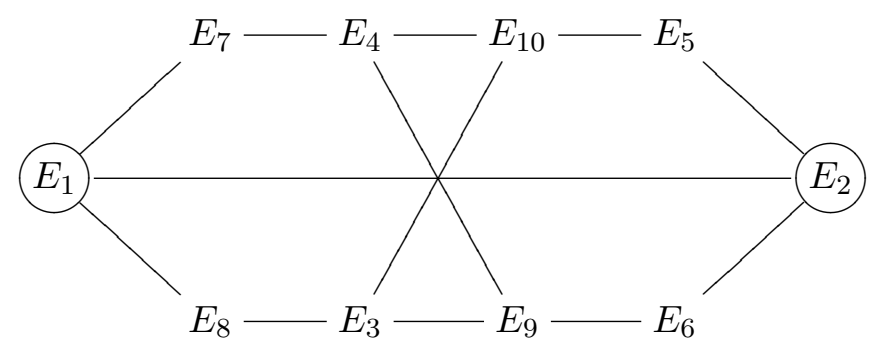

- The anticanonical map $\phi$ is given by

$$
\begin{aligned}
\left(\phi^{*}\left(x_{i}\right)\right)= & \left(\eta^{(0,0,1,1,0,0)} \eta_{9} \eta_{10}, \eta^{(1,1,0,1,0,1)} \eta_{7} \eta_{9}, \eta^{(1,1,1,0,1,0)} \eta_{8} \eta_{10},\right. \\
& \left.\eta^{(1,1,1,0,0,1)} \eta_{8} \eta_{9}, \eta^{(1,1,0,1,1,0)} \eta_{7} \eta_{10}\right),
\end{aligned}
$$

and $\phi^{*}\left(x_{1}+x_{3}\right)=\eta^{(1,2,0,0,1,2)} \eta_{9}, \phi^{*}\left(x_{1}+x_{4}\right)=\eta^{(2,1,0,1,0,0)} \eta_{7}^{2} \eta_{8}$, $\phi^{*}\left(x_{2}+x_{3}\right)=\eta^{(2,1,1,0,0,0)} \eta_{7} \eta_{8}^{2}, \phi^{*}\left(x_{2}+x_{4}\right)=\eta^{(1,2,0,0,2,1)} \eta_{10}, \phi^{*}\left(x_{1}+\right.$ $\left.x_{2}+x_{3}+x_{4}\right)=\eta^{(2,2,0,0,1,1)} \eta_{7} \eta_{8}$.

- Contracting one of $E_{5}, E_{6}, E_{7}, E_{8}$, we get a quintic surface of type $i\left(\mathbf{A}_{1}\right)$. For one of $E_{3}, E_{4}, E_{9}, E_{10}$, we obtain a quintic surface of type iii $\left(\mathbf{A}_{2}\right)$. 
- Let $Z_{5}$ be the affine variety defined by

$$
\begin{aligned}
& R_{1}=\eta_{6} \eta_{9}+\eta_{5} \eta_{10}-\eta_{1} \eta_{7} \eta_{8}, \\
& R_{2}=\eta_{4} \eta_{7}+\eta_{3} \eta_{8}-\eta_{2} \eta_{5} \eta_{6} .
\end{aligned}
$$

On the complement $U$ of $V=\left\{\eta_{3}=\cdots=\eta_{10}=0\right\}$, we have $\mathbb{A}\left(\widetilde{S}_{5}\right)=Z_{5}$. For $U^{\prime}=\left\{\eta_{3} \neq 0, \eta_{4}=\cdots=\eta_{10}=0\right\} \subset U$, we have $\mathbb{A}\left(\widetilde{S}_{5}\right) \cap U^{\prime}=Z_{5} \cap U^{\prime}=U^{\prime}$. Therefore, $\mathbb{A}\left(\widetilde{S}_{5}\right) \cap V=V$, which coincides with $Z_{5} \cap V$, and we obtain $\mathbb{A}\left(\widetilde{S}_{5}\right)=Z_{5}$.

\section{Type viii $\left(\mathbf{A}_{3}\right)$.}

- When we contract any of the four $(-1)$-curves $\widetilde{S}_{5}$, we obtain the minimal desingularization $\widetilde{S}_{4}$ of a quintic Del Pezzo surface $S_{4}$ of type iii $\left(\mathbf{A}_{2}\right)$, where $\operatorname{Cox}\left(\widetilde{S}_{4}\right)$ has two extra generators $\alpha_{1}, \alpha_{2}$. In total, we obtain four extra generators of $\operatorname{Cox}\left(\widetilde{S}_{5}\right)$.

- Its equation is:

$$
x_{0} x_{1}-x_{2}^{2}=\left(x_{0}+x_{1}+x_{3}\right) x_{3}-x_{2} x_{4}=0
$$

- Singularities: $(0: 0: 0: 0: 1)$ giving $E_{1}, E_{2}, E_{3}$; Lines: $E_{4}=\left\{x_{0}=\right.$ $\left.x_{2}=x_{3}=0\right\}, E_{5}=\left\{x_{1}=x_{2}=x_{3}=0\right\}, E_{6}=\left\{x_{0}=x_{2}=\right.$ $\left.x_{1}+x_{3}=0\right\}, E_{7}=\left\{x_{1}=x_{2}=x_{0}+x_{3}=0\right\}$

- A basis of $\operatorname{Pic}(\widetilde{S})$ is $E_{1}, \ldots, E_{6}$, with $E_{7}=(-1,0,1,-1,1,1)$, and $-K_{\widetilde{S}_{5}}=(1,2,3,0,2,2)$.

- Dynkin diagram:

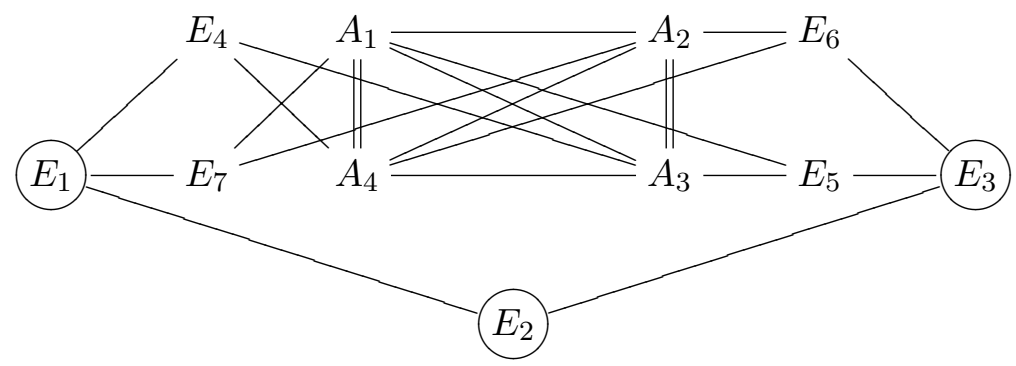

- The anticanonical map $\phi$ is given by

$$
\left(\phi^{*}\left(x_{i}\right)\right)=\left(\eta^{(1,2,3,0,2,2)}, \eta^{(3,2,1,2,0,0)} \eta_{7}^{2}, \eta^{(2,2,2,1,1,1)} \eta_{7}, \eta^{(1,1,1,0,1,0)} \eta_{7} \alpha_{1}, \alpha_{1} \alpha_{4}\right),
$$

and furthermore, $\phi^{*}\left(x_{1}+x_{3}\right)=\eta^{(1,1,1,0,0,1)} \eta_{7} \alpha_{2}, \phi^{*}\left(x_{0}+x_{3}\right)=$ $\eta^{(1,1,1,1,1,0)} \alpha_{3}, \phi^{*}\left(x_{0}+x_{1}+x_{3}\right)=\eta^{(1,1,1,1,0,1)} \alpha_{4}, \phi^{*}\left(x_{2}+x_{4}\right)=\alpha_{2} \alpha_{3}$.

- Relations: Let $V_{5} \subset \mathbb{A}^{11}$ be the affine variety defined by the five relations

$$
\begin{aligned}
& R_{1}=\eta_{1}^{2} \eta_{2} \eta_{4}^{2} \eta_{7}+\eta_{5} \alpha_{1}-\eta_{6} \alpha_{2}, \\
& R_{2}=\eta_{2} \eta_{3}^{2} \eta_{5}^{2} \eta_{6}+\eta_{7} \alpha_{2}-\eta_{4} \alpha_{4}, \\
& R_{3}=\eta_{2} \eta_{3}^{2} \eta_{5} \eta_{6}^{2}+\eta_{7} \alpha_{1}-\eta_{4} \alpha_{3}, \\
& R_{4}=\eta_{1}^{2} \eta_{2} \eta_{4} \eta_{7}^{2}+\eta_{5} \alpha_{3}-\eta_{6} \alpha_{4}, \\
& R_{5}=\eta_{1}^{2} \eta_{2}^{2} \eta_{3}^{2} \eta_{4} \eta_{5} \eta_{6} \eta_{7}+\alpha_{1} \alpha_{4}-\alpha_{2} \alpha_{3} .
\end{aligned}
$$

Note that $R_{1}, \ldots, R_{4}$ correspond to the relation in $\operatorname{Cox}\left(\widetilde{S}_{4}\right)$ when contracting $E_{4}, \ldots, E_{7}$, respectively. Therefore, $\operatorname{Cox}\left(\widetilde{S}_{5}\right)$ is given by 
$R_{1}, \ldots, R_{4}$ on $U$, the complement of $V=\left\{\eta_{4}=\eta_{5}=\eta_{6}=\eta_{7}=0\right\}$. As expected, $R_{5}$ follows from $R_{1}, \ldots, R_{4}$ on $U$.

However, when restricted to $V$, the relations $R_{1}, \ldots, R_{4}$ are trivial, while $R_{5}$ is $\alpha_{1} \alpha_{4}-\alpha_{2} \alpha_{3}=0$. As $\mathbb{A}\left(\widetilde{S}_{5}\right)$ is closed and irreducible, and $R_{5}$ holds on $U$, it must also hold on $V$, so $\mathbb{A}\left(\widetilde{S}_{5}\right) \subset$ $Z_{5}$. This shows that we need the extra relation $R_{5}$ in order to describe the closure of $Z_{5} \cap U$.

With $U^{\prime}=\left\{\eta_{4} \neq 0, \eta_{5}=\eta_{6}=\eta_{7}=0\right\} \subset U$, we have

$$
\mathbb{A}\left(\widetilde{S}_{5}\right) \cap U^{\prime}=Z_{5} \cap U^{\prime}=\left\{\eta_{4} \neq 0, \alpha_{3}=\alpha_{4}=\eta_{5}=\eta_{6}=\eta_{7}=0\right\} .
$$

Taking the closure, we conclude that $\mathbb{A}\left(\widetilde{S}_{5}\right) \cap V$ must contain $\left\{\alpha_{3}=\right.$ $\left.\alpha_{4}=\eta_{4}=\cdots=\eta_{7}=0\right\}$. Replacing $\eta_{4}$ by $\eta_{5}, \eta_{6}$ or $\eta_{7}$ in the definition of $U^{\prime}$, we see that $\mathbb{A}\left(\widetilde{S}_{5}\right) \cap V$ must contain all points of $V$ where one of $\alpha_{1}, \alpha_{4}$ and one of $\alpha_{2}, \alpha_{3}$ vanishes. This is equivalent to the condition $\alpha_{1} \alpha_{4}-\alpha_{2} \alpha_{3}=0$, which is $R_{5}$ restricted to $V$. Hence, no further relations are necessary to describe $\mathbb{A}\left(\widetilde{S}_{5}\right)$, so $\mathbb{A}\left(\widetilde{S}_{5}\right)=Z_{5}$.

\subsection{Degree 3}

In Section 5.6, the Cox ring of cubic surfaces is determined in every case where it has at most one relation. In this section, we determine generators of the Cox ring of singular cubic Del Pezzo surfaces in the remaining twelve of 20 cases.

\begin{tabular}{|c||c|c|c|c|}
\hline type & singularities & lines & extra generators & generators \\
\hline \hline 0 & - & 27 & - & 27 \\
$i$ & $\mathbf{A}_{1}$ & 21 & - & 22 \\
ii & $2 \mathbf{A}_{1}$ & 16 & - & 18 \\
$i i i$ & $\mathbf{A}_{2}$ & 15 & - & 17 \\
iv & $3 \mathbf{A}_{1}$ & 12 & - & 15 \\
$v$ & $\mathbf{A}_{2}+\mathbf{A}_{1}$ & 11 & - & 14 \\
vi & $\mathbf{A}_{3}$ & 10 & - & 13 \\
vii & $4 \mathbf{A}_{1}$ & 9 & - & 13 \\
viii & $\mathbf{A}_{2}+2 \mathbf{A}_{1}$ & 8 & - & 12 \\
ix & $\mathbf{A}_{3}+\mathbf{A}_{1}$ & 7 & - & 11 \\
$x$ & $2 \mathbf{A}_{2}$ & 7 & - & 11 \\
xi & $\mathbf{A}_{4}$ & 6 & 2 & 12 \\
xii & $\mathbf{D}_{4}$ & 6 & - & 10 \\
xiii & $\mathbf{A}_{3}+2 \mathbf{A}_{1}$ & 5 & - & 10 \\
xiv & $2 \mathbf{A}_{2}+\mathbf{A}_{1}$ & 5 & - & 10 \\
xv & $\mathbf{A}_{4}+\mathbf{A}_{1}$ & 4 & 1 & 10 \\
xvi & $\mathbf{A}_{5}$ & 3 & 4 & 12 \\
xvii & $\mathbf{D}_{5}$ & 3 & 2 & 10 \\
xviii & $3 \mathbf{A}_{2}$ & 3 & - & 9 \\
xix & $\mathbf{A}_{5}+\mathbf{A}_{1}$ & 2 & 2 & 10 \\
xx & $\mathbf{E}_{6}$ & 1 & 3 & 10 \\
\hline
\end{tabular}

TABle 6.3. Del Pezzo surfaces of degree 3 
For types $i$ to $x$, i.e., generalized Del Pezzo surfaces $\widetilde{S}_{6}$ whose types are $\mathbf{A}_{1}, 2 \mathbf{A}_{1}, \mathbf{A}_{2}, 3 \mathbf{A}_{1}, \mathbf{A}_{1}+\mathbf{A}_{2}, \mathbf{A}_{3}, 4 \mathbf{A}_{1}, \mathbf{A}_{2}+2 \mathbf{A}_{1}, \mathbf{A}_{3}+\mathbf{A}_{1}$ or $2 \mathbf{A}_{2}$, we check that the Cox ring is generated by non-zero global sections of the negative curves on $\widetilde{S}_{6}$.

The remaining two cases $x i$ and $x v i$ with singularities of type $\mathbf{A}_{4}$ and $\mathbf{A}_{5}$, respectively, are more interesting. We give more details in these cases.

In several cases, the easiest way to describe the extended Dynkin diagram is to explain how the diagram of a quartic Del Pezzo surface (see Section 6.4) must be modified in order to obtain the cubic surface.

Type i $\left(\mathbf{A}_{1}\right)$.

- The $(-2)$-curve $E_{0}$ intersects the $(-1)$-curves $E_{1}, \ldots, E_{6}$. There are 15 further $(-1)$-curves $E_{i, j}(i \neq j \in\{1, \ldots, 6\})$ which intersect $E_{i}, E_{j}$ and six curves $E_{k, l}$, where $\{i, j\} \cap\{k, l\}=\emptyset$.

Type ii $\left(2 \mathbf{A}_{1}\right)$.

- Blowing up the intersection of two $(-1)$-curves $E_{1}, E_{2}$ on a smooth quartic Del Pezzo surface $S_{5}$ results in the following Dynkin diagram of $\widetilde{S}_{6}$ :

On $S_{5}$, for $i \in\{1,2\}$, the (-2)-curve $E_{i}$ intersects four (-1)curves $E_{i, 1}, \ldots, E_{i, 4}$. There are six further $(-1)$-curves $E_{1}^{\prime}, \ldots, E_{6}^{\prime}$. Each (-1)-curve intersects four others.

In order to obtain the Dynkin diagram of $\widetilde{S}_{6}$, we change $E_{1}, E_{2}$ into $(-2)$-curves and remove the edge between them. Two new $(-1)$-curves $E_{3}, E_{4}$ are added, which intersect $E_{1}, E_{2}, E_{4}$ respectively $E_{1}^{\prime}, \ldots, E_{6}^{\prime}, E_{3}$.

Type iii $\left(\mathbf{A}_{2}\right)$.

- The Dynkin diagram of eight of the 17 negative curves is:

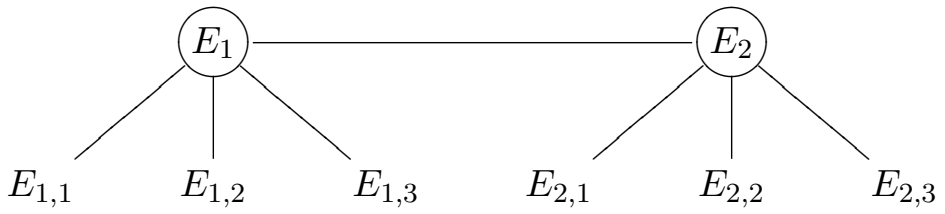

Furthermore, the nine (-1)-curves $E_{i, j}^{\prime}$ (where $i, j \in\{1,2,3\}$ ) intersect $E_{1, i}, E_{2, j}$ and the four curves $E_{i^{\prime}, j^{\prime}}^{\prime}$, where $i \neq i^{\prime}$ and $j \neq j^{\prime}$.

Type iv $\left(3 \mathbf{A}_{1}\right)$.

- Blowing up the intersection of $E_{1,1}$ and $E_{2,2}$ on the quartic type $i$ $\left(\mathbf{A}_{1}\right)$ surface gives the following Dynkin diagram: We turn $E_{1,1}$ and $E_{2,2}$ into $(-2)$-curves and remove the edge between them. Two new $(-1)$-curves $E, E^{\prime}$ are added, intersecting $E_{1,1}, E_{2,2}, E^{\prime}$ respectively $E_{1,2}, E_{2,1}, E_{3}, E_{4}, E$ (the $(-1)$ curves which do not intersect $E_{1,1}$ or $\left.E_{2,2}\right)$.

Type v $\left(\mathbf{A}_{1}+\mathbf{A}_{2}\right)$.

- Blowing up the intersection of $E_{1}$ and $E_{1,1}$ on the quartic type $i$ $\left(\mathbf{A}_{1}\right)$ surface gives the following Dynkin diagram: We turn $E_{1}$ to 
$E_{1,1}$ into (-2)-curves and remove the edge between them. A new $(-1)$-curve $E$ intersects $E_{1}$ and $E_{1,1}$.

\section{Type vi $\left(\mathbf{A}_{3}\right)$.}

- The extended Dynkin diagram is the following modification of the diagram of quartic type $i i$ (2 $\mathbf{A}_{1}$ with 9 lines), corresponding to the blow-up of a point on $E_{3}$ : The $(-1)$-curve $E_{3}$ is changed to a $(-2)$-curve. Two new $(-1)$-curves $E$ and $E^{\prime}$ are added, where $E$ intersects $E_{3}, E^{\prime}$, and $E^{\prime}$ intersects $E_{8}, E_{9}, E_{10}, E_{11}, E$.

\section{Type vii $\left(4 \mathbf{A}_{1}\right)$.}

- See Example 6.7 .

Type viii $\left(\mathbf{A}_{2}+2 \mathbf{A}_{1}\right)$.

- Dynkin diagram:

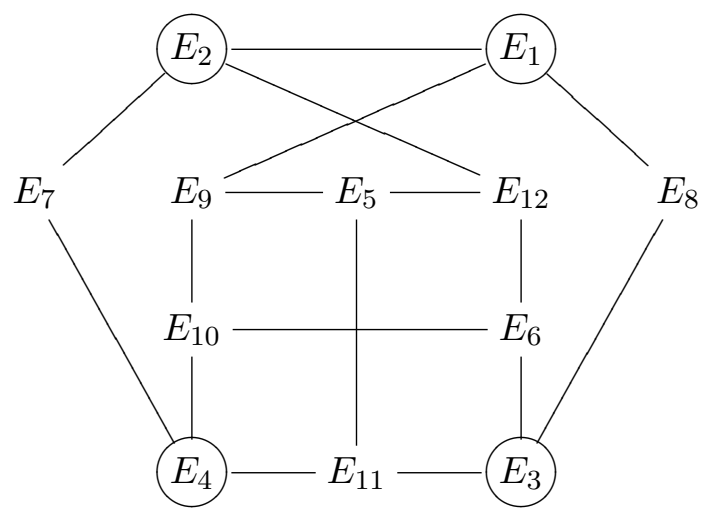

Type ix $\left(\mathbf{A}_{3}+\mathbf{A}_{1}\right)$.

- Dynkin diagram:

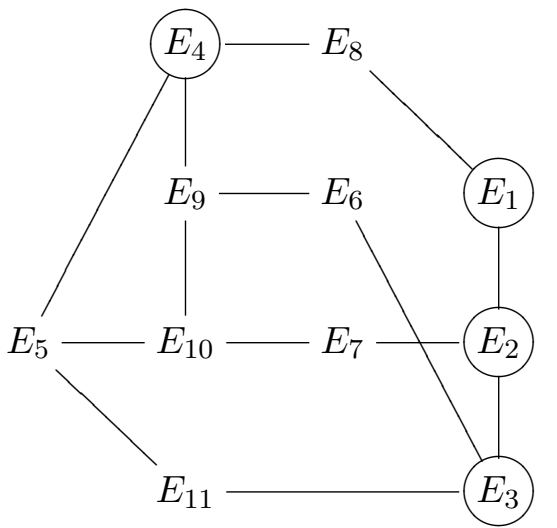

Type x (2A $\left.\mathbf{A}_{2}\right)$.

- See Section 6.6.

Type xi $\left(\mathrm{A}_{4}\right)$. 
- Dynkin diagram:

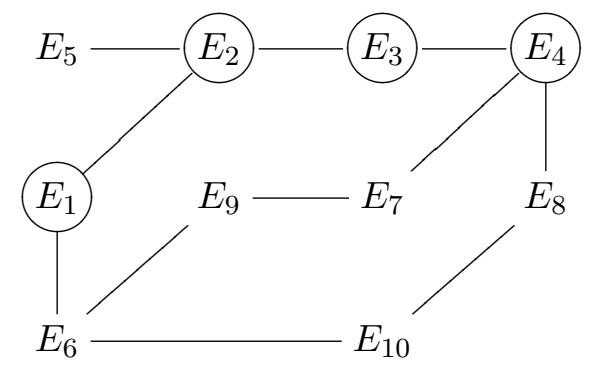

- We claim that $\operatorname{Cox}\left(\widetilde{S}_{6}\right)$ is generated by $\eta_{1}, \ldots, \eta_{10}$ and $\alpha_{1}, \alpha_{2}$, where $A_{1}:=\operatorname{deg}\left(\alpha_{1}\right)$ intersects $E_{5}, E_{7}, E_{9}, A_{2}$ and twice $E_{10}$, while $A_{2}:=$ $\operatorname{deg}\left(\alpha_{2}\right)$ intersects $E_{5}, E_{8}, E_{10}, A_{1}$ and twice $E_{9}$. This is checked by considering the $\operatorname{Cox}\left(\widetilde{S}_{5}\right)$ for the various quartic contractions $\widetilde{S}_{5}$ of the $(-1)$-curves on $\widetilde{S}_{6}$ :

\begin{tabular}{|c|c|c|c|}
\hline$(-1)$-curve & type of $S_{5}$ & extra curves on $\widetilde{S}_{5}$ & corresp. curves on $\widetilde{S}_{6}$ \\
\hline \hline$E_{5}$ & vi: $\mathbf{A}_{2}+\mathbf{A}_{1}$ & - & - \\
$E_{6}$ & viii $: \mathbf{A}_{3}$ & $A_{1}, A_{2}, A_{3}, A_{4}$ & $A_{1}, E_{10}, A_{2}, E_{9}$ \\
$E_{7}$ & vii $: \mathbf{A}_{3}$ & $A$ & $A_{2}$ \\
$E_{8}$ & vii $: \mathbf{A}_{3}$ & $A$ & $A_{1}$ \\
$E_{9}$ & xii $: \mathbf{A}_{4}$ & $A_{1}, A_{2}$ & $A_{2}, E_{6}$ \\
$E_{10}$ & xii $: \mathbf{A}_{4}$ & $A_{1}, A_{2}$ & $A_{1}, E_{6}$ \\
\hline
\end{tabular}

Type xvi $\left(A_{5}\right)$.

- Dynkin diagram:

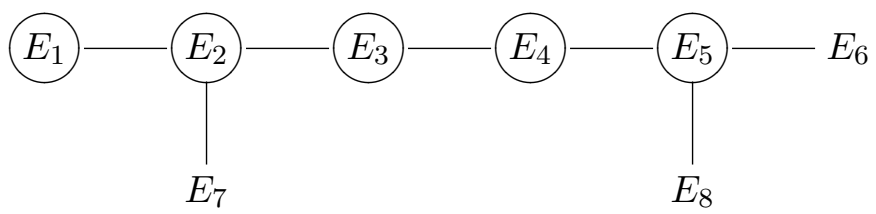

- Contracting $E_{6}$ gives a quartic surface $\widetilde{S}_{5}$ with a singularity of type $\mathbf{A}_{4}$. We have two extra generators $\alpha_{1}, \alpha_{2}$ of degree $A_{1}, A_{2}$ which intersect $E_{7}$ and twice $E_{8}$ respectively $E_{1}$ and $E_{8}$. Contracting $E_{8}$ gives the same type of quartic surface. Here, the extra generators intersect $E_{7}$ and twice $E_{6}$ respectively $E_{1}$ and $E_{6}$. This shows that we need four extra generators $\alpha_{1}, \ldots, \alpha_{4}$ such that the corresponding divisors $A_{1}, \ldots, A_{4}$ intersect one of $E_{1}, E_{7}$ and one of $E_{6}, E_{8}$.

Contracting $E_{7}$ gives a quartic surface with singularities $\mathbf{A}_{3}+$ $\mathbf{A}_{1}$. Its Cox ring also has two extra generators, but the induced sections of $\widetilde{S}_{6}$ are two of $\alpha_{1}, \ldots, \alpha_{4}$ as above.

- Therefore, $\operatorname{Cox}\left(\widetilde{S}_{6}\right)$ is generated by these 12 elements. By considering the sections in degrees $A_{i}$, we see that it is a minimal generating set (cf. Lemma 2.5).

\subsection{Families of Del Pezzo surfaces}

In this section we compute the Cox ring of a family of Del Pezzo surfaces. By Example 1.36, there is a one-parameter family of singular Del 
Pezzo surfaces $S$ of type $2 \mathbf{A}_{2}$. Its extended Dynkin diagram is given in Example 1.32 .

We have $\operatorname{dim}\left(H^{0}\left(l_{0}-l_{5}\right)\right)=2$, but

$$
l_{0}-l_{5}=E_{4}+E_{6}+2 E_{7}=E_{1}+E_{11}=E_{2}+E_{8}=E_{3}+E_{9} .
$$

Hence, there are two relations between

$$
\eta_{4} \eta_{6} \eta_{7}^{2}, \quad \eta_{1} \eta_{11}, \quad \eta_{2} \eta_{8}, \quad \eta_{3} \eta_{9}
$$

where $\eta_{i}$ is a non-zero element of $H^{0}\left(E_{i}\right)$. To determine the relation between these sections, note that

$$
\eta_{4} \eta_{5} \eta_{6} \eta_{7}^{2}, \quad \eta_{1} \eta_{5} \eta_{11}, \quad \eta_{2} \eta_{5} \eta_{8}, \quad \eta_{3} \eta_{5} \eta_{9} \in H^{0}\left(\widetilde{S}_{6}, l_{0}\right)
$$

are $\pi^{*}\left(s_{7}\right), \ldots, \pi^{*}\left(s_{10}\right)$ under the map $\pi: S_{6} \rightarrow \mathbb{P}^{2}$, with $s_{i} \in H^{0}\left(\mathbb{P}^{2}, \mathcal{O}_{\mathbb{P}^{2}}(1)\right)$ is a section vanishing in $E_{i}$ for $i \in\{7, \ldots, 10\}$. By our choice of $E_{7}, \ldots, E_{10}$ in $\mathbb{P}^{2}$ as in (1.1), depending on the parameter $\alpha$, two relations between $s_{7}, \ldots, s_{10}$ are

$$
s_{9}+s_{8}-s_{7}=0 \text { and } s_{10}+\alpha s_{8}-s_{7}=0 .
$$

This gives rise to two relations in $H^{0}\left(\widetilde{S}_{6}, l_{0}\right)$, which results in relations in $H^{0}\left(\widetilde{S}_{6}, l_{0}-l_{5}\right)$ after dividing by $\eta_{5}$ :

$R_{1}\left(\eta_{i}\right):=\eta_{2} \eta_{8}+\eta_{1} \eta_{11}-\eta_{4} \eta_{6} \eta_{7}^{2}=0, \quad R_{2}\left(\eta_{i}\right):=\eta_{3} \eta_{9}+\alpha \eta_{1} \eta_{11}-\eta_{4} \eta_{6} \eta_{7}^{2}=0$.

Note that the contraction of the $(-1)$-curve $E_{7}$ on $\widetilde{S}_{6}$ results in a generalized quartic Del Pezzo surface $\widetilde{S}_{5}$ of type $2 \mathbf{A}_{1}$ (with 8 lines), while the contraction of any other (-1)-curve gives $\widetilde{S}_{5}$ of type $\mathbf{A}_{2}+\mathbf{A}_{1}$. As before, we can check by comparing the generators $\eta_{1}, \ldots, \eta_{11}$ and the relations $R_{1}$, $R_{2}$ with the results in these two quartic types that we have

$$
\operatorname{Cox}\left(\widetilde{S}_{6}\right)=\mathbb{K}\left[\eta_{1}, \ldots, \eta_{11}\right] /\left(R_{1}\left(\eta_{i}\right), R_{2}\left(\eta_{i}\right)\right) .
$$

Our method of determining the Cox ring shows that the following properties are invariant in families of isomorphy classes $\widetilde{S}$ of the same type:

- By definition, the extended Dynkin diagram and the Picard group.

- The numbers and degrees of the generators of $\operatorname{Cox}(\widetilde{S})$ in $\operatorname{Pic}(\widetilde{S})$ (see Lemma 2.5).

- The numbers and degrees of the relations in $\operatorname{Pic}(\widetilde{S})$.

However, the exact form of the relations depends on the choice of isomorphy class in the family. As the monomials occurring in a relation are determined by the degrees of the relation and the generators, only the coefficients vary.

See also Section 5.6 for the two isomorphy classes of type $\mathbf{D}_{4}$ in degree 3. 

Part 2

Rational points on Del Pezzo surfaces 



\section{CHAPTER 7}

\section{Manin's conjecture}

\subsection{Introduction}

Let $f \in \mathbb{Z}\left[x_{0}, \ldots, x_{n}\right]$ be a non-singular form of degree $d$. By the circle method,

$$
N(f, B):=\#\left\{\mathbf{x} \in \mathbb{Z}^{n+1} / \pm \mid \max _{j}\left(\left|x_{j}\right|\right) \leq B\right\} \sim c \cdot B^{n+1-d}
$$

(where $\mathbf{x} \in \mathbb{Z}^{n+1} / \pm$ means that we identify $\mathbf{x}$ with $-\mathbf{x}=\left(-x_{0}, \ldots,-x_{n}\right)$ ) with $c \in \mathbb{R}_{>0}$, provided that $n>2^{d} \cdot(d-1)$, and $f(\mathbf{x})=0$ has solutions over all completions of $\mathbb{Q}$ (see $[$ Bir62 $)$. Let $X=X_{f} \subset \mathbb{P}^{n}$ be the smooth hypersurface over $\mathbb{Q}$, given by $f(\mathbf{x})=0$. It follows that

$$
N_{X, H_{-K_{X}}}(B):=\#\left\{\mathbf{x} \in X(\mathbb{Q}) \mid H_{-K_{X}}(\mathbf{x}) \leq B\right\} \sim C \cdot B,
$$

as $B \rightarrow \infty$. Here $X(\mathbb{Q})$ is the set of rational points on $X$, represented by primitive vectors $\mathbf{x} \in\left(\mathbb{Z}_{\text {prim }}^{n+1} \backslash 0\right) / \pm$ (i.e., $\mathbf{x}=\left(x_{0}, \ldots, x_{n}\right)$ is identified with $-\mathbf{x}$, and there is no prime dividing all coordinates $\left.x_{0}, \ldots, x_{n}\right)$, and

$$
H_{-K_{X}}(\mathbf{x}):=\max _{j}\left(\left|x_{j}\right|\right)^{n+1-d}, \text { for } \mathbf{x}=\left(x_{0}, \ldots, x_{n}\right) \in\left(\mathbb{Z}_{\text {prim }}^{n+1} \backslash 0\right) / \pm
$$

is the anticanonical height of a primitive representative.

The main goal of Manin's program on the distribution of rational points of bounded height (see [FMT89] and [BM90]) is an extension of the asymptotic formula (7.1) to Fano varieties which are not necessarily isomorphic to hypersurfaces in projective space. A Fano variety is a projective variety whose anticanonical class is ample.

It became apparent that, in general, to obtain a geometric interpretation of asymptotic results, it may be necessary to restrict to appropriate Zariski open subsets $U$ of $X$. Otherwise, the number of rational points on a Zariski closed subset of lower dimension may dominate the total number of rational points; e.g., this phenomenon occurs for smooth and for singular cubic surfaces where the number of rational points on rational lines on such a surface dominates the total number of rational points. Hence, we count rational points on the complement of these lines. It is often necessary to consider rational points in finite extensions of the rationals: while $X(\mathbb{Q})$ might be empty, $X(k)$ could still contain infinitely many points for some number field $k$.

We are concerned with Del Pezzo surfaces, cf. Chapter 1 for their basic properties. Smooth Del Pezzo surfaces are birational to $\mathbb{P}^{2}$, provided the ground field is algebraically closed. Besides $\mathbb{P}^{1} \times \mathbb{P}^{1}$, they are blow-ups of $\mathbb{P}^{2}$ in at most eight points in general position. 
Of special interest are irreducible curves for which the self intersection number is negative, which are called negative curves (Definition 1.20). Arithmetically, rational points tend to accumulate on negative curves where they are easy to count. Our main focus is to count rational points on the complement of the negative curves.

For smooth Del Pezzo surfaces of degree $\geq 3$, the negative curves are exactly the lines (in the anticanonical embedding, see Remark 1.13), having self intersection number -1 .

Singular Del Pezzo surfaces are obtained as described in Section 1.5. We blow up $\mathbb{P}^{2}$ in points in almost general position (see Definition 1.20 e.g., three points on a line). This results in a generalized Del Pezzo surface, i.e., a smooth surface $\widetilde{S}$ containing negative curves with self intersection number -2 (called (-2)-curves). Contracting the $(-2)$-curves gives a singular Del Pezzo surface $S$ whose singularities are rational double points and whose minimal desingularization is $\widetilde{S}$.

For number fields, we say that a Del Pezzo surface is split if all of the negative curves are defined over that ground field, in which case the surface is birational to $\mathbb{P}^{2}$. There do exist non-split Del Pezzo surfaces which are birational to $\mathbb{P}^{2}$ over that ground field; however, the generic Del Pezzo surface is non-split and is not birational to $\mathbb{P}^{2}$ over the ground field.

From here, we work over $\mathbb{Q}$. Manin's conjecture in the special case of smooth or singular Del Pezzo surfaces $S_{r}$ of degree $9-r \geq 3$ can be formulated as follows. Consider $S_{r}$ as a subvariety of $\mathbb{P}^{9-r}$ using the anticanonical embedding (Remark 1.13 and Remark 1.26). For the height function on $\mathbb{P}^{9-r}(\mathbb{Q})$, we always use

$$
H(\mathbf{x}):=\max \left(\left|x_{0}\right|, \ldots,\left|x_{9-r}\right|\right),
$$

with $\mathbf{x} \in \mathbb{P}^{9-r}(\mathbb{Q})$ represented by integral coprime coordinates $x_{0}, \ldots, x_{9-r}$. For an open subset $U$ of $S$, let

$$
N_{U, H}(B):=\#\{\mathbf{x} \in S(\mathbb{Q}) \mid H(\mathbf{x}) \leq B\} .
$$

ConjeCture 7.1. Let $S$ be a smooth or singular Del Pezzo surface over $\mathbb{Q}$. Then there exists a subset $U \subset S$ which is dense and open in the Zariski topology such that

$$
N_{U, H}(B) \sim c_{S, H} \cdot B(\log B)^{t-1},
$$

as $B \rightarrow \infty$, where $t$ is the rank of the Picard group over $\mathbb{Q}$ of the minimal desingularization $\widetilde{S}$ of $S$.

The constant $c_{S, H}$ has been defined by Peyre Pey95; see Section 7.2 for more details. It should be non-zero if $S(\mathbb{Q}) \neq \emptyset$. It is analogous to the singular series and the singular integral that you meet in the classical circle method. Note that a line defined over $\mathbb{Q}$ on a Del Pezzo surface of degree $\geq 3$ contributes $\sim c \cdot B^{2}$ rational points to the counting function (for some positive constant $c$ ). Thus it is expected that $U$ is the complement to all lines defined over $\mathbb{Q}$ (i.e., the negative curves).

Table 7.1 gives an overview of current results towards Conjecture 7.1 for Del Pezzo surfaces. In Column 4 ("type of result"), "asymptotic" means that the analog of $(7.3)$ is established, including the predicted value of the constant; "bounds" means that only upper and lower bounds of the expected 
order of magnitude $B(\log B)^{t}$ are known. As explained in Definition 1.28, the type of a surface is usually denoted by the Dynkin diagram associated to its singularities.

\begin{tabular}{|c|c|c|c|c|}
\hline degree & singularities & (non-)split & type of result & reference \\
\hline$\geq 6$ & - & split & asymptotic & BT98 \\
\hline 5 & - & split & asymptotic & Bre02 \\
\hline 5 & - & non-split & asymptotic & BF04 \\
\hline 4 & $\mathbf{D}_{5}$ & split & asymptotic & CLT02, [BB04 \\
\hline 4 & $\mathbf{D}_{4}$ & non-split & asymptotic & BB05 \\
\hline 4 & $\mathbf{D}_{4}$ & split & asymptotic & Chapter 10 \\
\hline 4 & $3 \mathbf{A}_{1}$ & split & bounds & Bro05 \\
\hline 4 & $2 \mathbf{A}_{1}$ & non-split & asymptotic & $\begin{array}{l}\text { de la Bretèche, } \\
\text { Browning, Peyre }\end{array}$ \\
\hline 3 & $3 \mathbf{A}_{2}$ & split & asymptotic & BT98, Bre98, . \\
\hline 3 & $4 \mathbf{A}_{1}$ & split & bounds & HB03 \\
\hline 3 & $\mathbf{D}_{4}$ & split & bounds & Bro04 \\
\hline 3 & $\mathbf{E}_{6}$ & split & asymptotic & Chapter 9, BBD05 \\
\hline
\end{tabular}

TABle 7.1. Results for Del Pezzo surfaces

The paper [BT98] contains a proof of Manin's conjecture for toric Fano varieties. This includes all smooth Del Pezzo surfaces of degree $\geq 6$ and the $3 \mathbf{A}_{2}$ cubic surface. A list of all types of toric Del Pezzo surfaces can be found in Table 1.4 .

Figure 7.1 shows all points of height $\leq 50$ on the Cayley cubic surface (Example 6.7), which has four singularities of type $\mathbf{A}_{1}$ and was considered in [HB03.

The proofs of Manin's conjecture proceed either via the height zeta function

$$
Z_{U, H}(s):=\sum_{\mathbf{x} \in U(\mathbb{Q})} H_{-K_{X}}(\mathbf{x})^{-s}
$$

whose analytic properties are related to the asymptotic 7.3 by Tauberian theorems (see Section 7.3 ), or via the lifting of the counting problem to the universal torsor - an auxiliary variety parameterizing rational points. Experience shows that counting points on the universal torsor is often easier. The torsor approach was developed by Colliot-Thélène and Sansuc in the context of the Brauer-Manin obstruction [CTS87] and was applied to Manin's conjecture by Peyre Pey98 and Salberger Sal98.

In the simplest case of hypersurfaces $X=X_{f} \subset \mathbb{P}^{n}$ over $\mathbb{Q}$, with $n \geq 4$, this is exactly the passage from rational vectors $\mathbf{x}=\left(x_{0}, \ldots, x_{n}\right)$, modulo the diagonal action of $\mathbb{Q}^{*}$, to primitive lattice points $\left(\mathbb{Z}_{\text {prim }}^{n+1} \backslash 0\right) / \pm$. Geometrically, we have

$$
\mathbb{A}^{n+1} \backslash\{0\} \stackrel{\mathbb{G}_{\mathrm{m}}}{\longrightarrow} \mathbb{P}^{n} \quad \text { and } \quad \mathcal{T}_{X} \stackrel{\mathbb{G}_{\mathrm{m}}}{\longrightarrow} X .
$$

Here, $\mathcal{T}_{X}$ is the hypersurface in $\mathbb{A}^{n+1} \backslash\{0\}$ defined by the form $f$. The 1dimensional torus $\mathbb{G}_{\mathrm{m}}$ is interpreted as the Néron-Severi torus $T_{\mathrm{NS}}(X)$ (see Section 2 since $\operatorname{Pic}(X)$ has rank 1 . Rational points on the base surface $X$ are lifted to integral points on the torsor, modulo the action of the group 


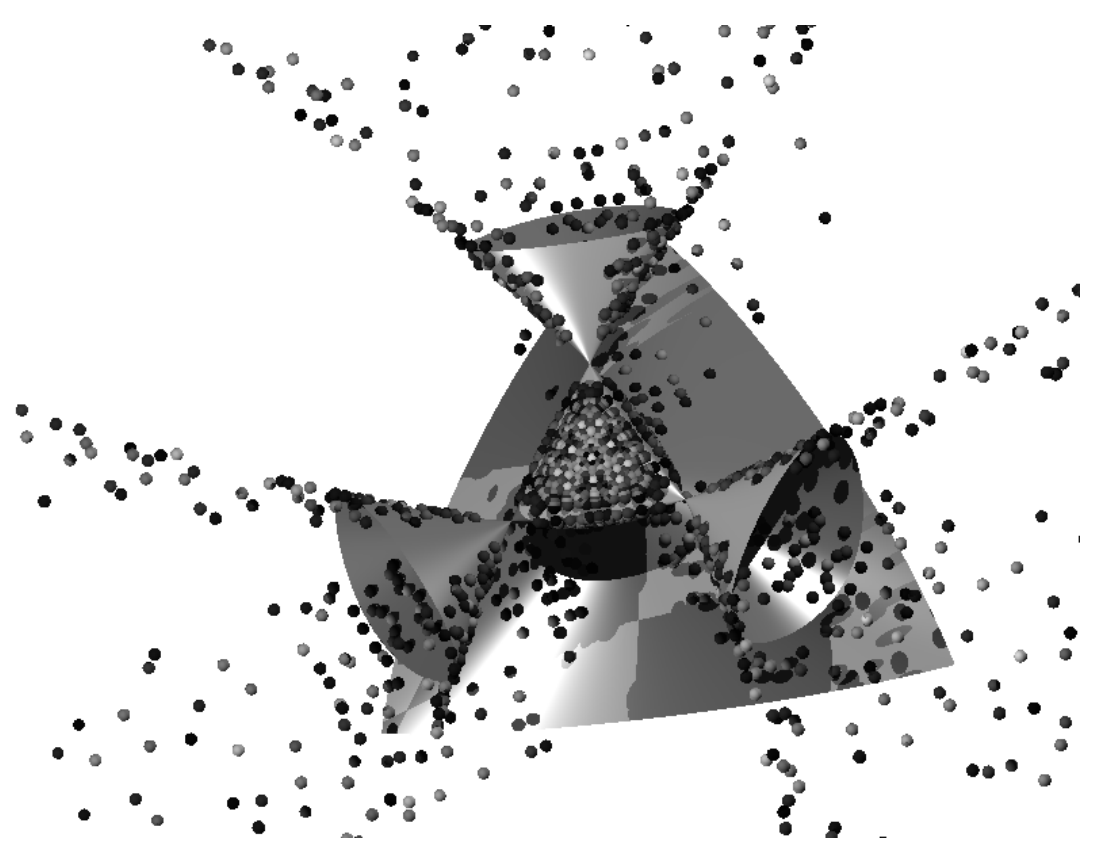

Figure 7.1. Points of height $\leq 50$ on the Cayley cubic surface $x_{0} x_{1} x_{2}+x_{0} x_{1} x_{3}+x_{0} x_{2} x_{3}+x_{1} x_{2} x_{3}=0$.

of units $T_{\mathrm{NS}}(X)(\mathbb{Z})=\{ \pm 1\}$. The height inequality $H(\mathbf{x}) \leq B$ for $\mathbf{x}$ on the base $X$ translates into the usual height inequality on the torsor $(7.2)$. In this case, it is possible to count the points on the torsor using the classical circle method.

For hypersurfaces in $\mathbb{P}^{3}$, or more generally for complete intersection surfaces, the Picard group may have higher rank. See Section 2.2 for the definition of universal torsors $\mathcal{T}_{S}$ of generalized Del Pezzo surfaces $S$ as $T_{\mathrm{NS}}(S)$ bundles over $S$. For example, for split smooth cubic surfaces the rank is 7 , so that the dimension of the corresponding universal torsor $\mathcal{T}_{S}$ is 9 ; for quartic Del Pezzo surfaces these are 6 and 8, respectively.

In order to prove Manin's conjecture (Conjecture 7.1) for Del Pezzo surfaces, rational points on $S$ are lifted to certain integral points on $\mathcal{T}_{S}$, modulo the action of $T_{\mathrm{NS}}(S)(\mathbb{Z})=( \pm 1)^{t}$, where $t$ is the rank of $\operatorname{Pic}(S)$. The height inequality on $S$ translates into appropriate inequalities on $\mathcal{T}_{S}$. This explains the interest in the geometry of torsors, and especially, in their equations. As we have seen in Part 1, the explicit determination of these equations is an interesting algebro-geometric problem.

Estimating the number of integral points subject to height inequalities on the universal torsor seems to be easiest for toric Del Pezzo surfaces since their universal torsors are open subsets of affine space. This was used in Salberger's proof of Manin's conjecture [Sal98] for all split toric varieties over $\mathbb{Q}$.

The easiest non-toric cases are singular Del Pezzo surfaces whose universal torsors are open subsets of hypersurfaces. A first example, a quartic Del Pezzo surface of type $\mathbf{D}_{5}$, was treated by de la Bretèche and Browning [BB04], while Chapter 9 gives a proof for the $\mathbf{E}_{6}$ cubic surface. A more 
detailed analysis of this surface, which gives a much improved error term in the asymptotic, can be found in a separate paper (joint with R. de la Bretèche and T. D. Browning [BBD05], see also Section 7.3).

While in principle, a similar approach seems possible for the other surfaces whose universal torsors are hypersurfaces, it does not seem to be easy to count the points on the universal torsor using methods of analytic number theory. The first step of passing to the universal torsor can be done using the detailed information on the Cox ring given in Chapter 5 for all singular Del Pezzo surfaces of degree $\geq 3$ which have such a universal torsor. One example which can be treated is a split quartic Del Pezzo surface of type $\mathbf{D}_{4}$, see Chapter 10.

However, new difficulties seem to arise in the estimation of error terms in many other cases. Apparently, it is easier for Del Pezzo surfaces of higher degree. In each degree, the easiest cases seem to be the ones with the "worst" singularities, e.g., $\mathbf{D}_{5}$ in degree 4 and $\mathbf{E}_{6}$ in degree 3 . Therefore, the next challenge would be to prove Manin's conjecture for a singular Del Pezzo surface of degree 2 , or for other singular cubic surfaces.

\subsection{Peyre's constant}

In this section, we explain the leading constant $c_{S, H}$ appearing in Conjecture 7.1 for a smooth Del Pezzo surface $S$ defined over a number field $\mathbb{K}$. It was suggested by Peyre Pey95, Batyrev and Tschinkel [BT95] and is defined as the product

$$
c_{S, H}:=\alpha(S) \cdot \beta(S) \cdot \omega_{H}(S)
$$

of the following three constants.

The first factor $\alpha(S)$ is related to a certain volume in the effective cone $\Lambda_{\text {eff }}(S)$ in the Picard group $\operatorname{Pic}(S)$. Note that the ground field is not necessarily algebraically closed, so the rank of $\operatorname{Pic}(S)$ could be lower than $r+1$ if $S$ is a Del Pezzo surface of degree $9-r$. For its precise definition and its calculation in the case of split Del Pezzo surfaces, see Chapter 8 below.

The second factor $\beta(S)$ is defined as the order of the Galois cohomology group

$$
H^{1}(\mathbb{K}, \operatorname{Pic}(S)):=H^{1}\left(\operatorname{Gal}(\overline{\mathbb{K}} / \mathbb{K}), \operatorname{Pic}(S)_{\overline{\mathbb{K}}}\right) .
$$

It first appeared in [BT95] and [BT98, Section 1]. We have $\beta(S)=1$ for split Del Pezzo surfaces.

For singular Del Pezzo surfaces, we must replace $\alpha(S)$ and $\beta(S)$ by $\alpha(\widetilde{S})$ and $\beta(\widetilde{S})$ for the minimal desingularization $\widetilde{S}$ of $S$.

Example 7.2. Consider a singular quartic Del Pezzo surface $S$ with two conjugate singularities which are not joined by a line on $S$. Such a surface is called Iskovskih surface (cf. [CT88, Section 7]). Its type is $2 \mathbf{A}_{1}$ (with eight lines). In yet unpublished work of de la Bretèche, Browning, and Peyre, Manin's conjecture is proved for an Iskovskih surface $S$ with $\beta(S)>1$.

The third factor $\omega_{H}(S)$ does not only depend on the surface $S$, but also on the height function $H$. If the ground field is $\mathbb{K}=\mathbb{Q}$, it is defined as the 
product

$$
\omega_{H}(S)=\lim _{s \rightarrow 1}\left((s-1)^{\mathrm{rk} \operatorname{Pic}(S)} L(s, \operatorname{Pic}(S))\right) \omega_{\infty}(S) \prod_{p} \frac{\omega_{p}(S)}{L_{p}(1, \operatorname{Pic}(S))} .
$$

Here, $\omega_{\infty}(S)$ is the "density of points over $\mathbb{R}$ " and can be calculated by Pey95, Section 5.1] as follows using the Leray form if $S$ is a complete intersection: Suppose the anticanonical embedding of $S$ is given by $m$ forms $f_{1}, \ldots, f_{m} \in \mathbb{Z}\left[x_{0}, \ldots, x_{N}\right]$ in $\mathbb{P}^{N}$ such that the matrix

$$
M(\mathbf{x}):=\left(\frac{\partial f_{i}}{\partial x_{j}}(\mathbf{x})\right)_{1 \leq i \leq m, N-m+1 \leq j \leq N}
$$

is invertible. Then

$$
\omega_{\infty}(S)=\int_{\left|x_{0}\right|, \ldots,\left|x_{N}\right| \leq 1} M(\mathbf{x})^{-1} \mathrm{~d} x_{0} \ldots \mathrm{d} x_{N-m} .
$$

For each prime $p$, the constant $\omega_{p}(S)$ is the " $p$-adic density" of points on $S$. It can be calculated using the Leray measure Pey95, Lemma 5.4.6]. For complete intersections $S$ in projective space $\mathbb{P}^{N}$, defined by $m$ forms $f_{1}, \ldots, f_{m} \in \mathbb{Z}\left[x_{0}, \ldots, x_{N}\right]$, [PT01a, Proposition 3.1] gives the following possibility of its explicit calculation:

$$
\omega_{p}(S)=\lim _{n \rightarrow \infty} \frac{\#\left\{\mathbf{x} \in\left(\mathbb{Z} / p^{n} \mathbb{Z}\right)^{N+1} \mid\left(f_{1}(\mathbf{x}), \ldots, f_{m}(\mathbf{x})\right)=0 \in\left(\mathbb{Z} / p^{n} \mathbb{Z}\right)^{m}\right\}}{p^{n \cdot(\operatorname{dim} S+1)}} .
$$

By Remark 1.13 and Remark 1.26, this includes the smooth and singular cubic and quartic Del Pezzo surfaces $S_{r}$, where $m=1, N=3$ in the cubic case, and $m=2, N=4$ in the quartic case. See [BB04] for an example of its calculation.

For the definition of the $L$-functions occuring in the definition of $\omega_{H}(S)$, see Pey95, Section 2.2]. In the split case, we have

$$
\lim _{s \rightarrow 1}\left((s-1)^{\mathrm{rk} \operatorname{Pic}(S)} L(s, \operatorname{Pic}(S))=\lim _{s \rightarrow 1}\left((s-1)^{r+1} \zeta(s)^{r+1}\right)=1\right.
$$

and

$$
L_{p}(1, \operatorname{Pic}(S))^{-1}=(1-1 / p)^{r+1} .
$$

For a singular Del Pezzo surface $S$ with minimal desingularization $\widetilde{S}$, we calculate $\omega_{H}(\widetilde{S})$, where the anticanonical image of $\widetilde{S}$ coincides with the anticanonical embedding of $S$.

\subsection{Height zeta functions}

Let $S$ be a smooth or singular Del Pezzo surface whose minimal desingularization is the generalized Del Pezzo surface $\widetilde{S}$ (with $\widetilde{S}=S$ in the smooth case). For simplicity, we assume that $S$ is split over $\mathbb{Q}$, with height function $H: S(\mathbb{Q}) \rightarrow \mathbb{R}$. Let $U$ be the complement of the lines on $S$.

Definition 7.3. The height zeta function $Z_{U, H}$ of $S$ is defined for all $s \in \mathbb{C}$ with real part $\Re e(s)>1$ as

$$
Z_{U, H}(s):=\sum_{\mathbf{x} \in U(\mathbb{Q})} \frac{1}{H(x)^{s}} .
$$


Conjecture 7.1 is related to properties of the height zeta function in the following way by Tauberian theorems:

- If

$$
N_{U, H}(B) \sim c_{S, H} \cdot B(\log B)^{t-1},
$$

then $Z_{U, H}(s)$ should have a pole of order $t$ at $s=1$ with residue $(t-1) ! \cdot c_{S, H}$.

- If we can prove Conjecture 7.1 with an error term of order $B^{1-\delta}$ for some $\delta>0$, i.e., if

$$
N_{U, H}(B)=c_{S, H} \cdot B Q(\log B)+O\left(B^{1-\delta}\right),
$$

where $Q$ is a monic polynomial of degree $r-1$, then $Z_{U, H}(s)$ should have a meromorphic continuation to the halfplane

$$
\{s \in \mathbb{C} \mid \Re e(s)>1-\delta\} .
$$

Our proofs of Manin's conjecture for a cubic surface of type $\mathbf{E}_{6}$ in Chapter 9 and a quartic surface of type $\mathbf{D}_{4}$ in Chapter 10 give error terms of order $O\left(B(\log B)^{2}\right)$ respectively $O\left(B(\log B)^{3}\right)$. This does not yield information on the properties of $Z_{U, H}(s)$ for $\Re e(s)<1$.

However, in joint work with de la Bretèche and Browning [BBD05], we prove the following result:

TheOrem 7.4. Let $S$ be the cubic surface of type $\mathbf{E}_{6}$ as in (9.1), and let $U$ be the complement of the unique line on $S$. Then

$$
N_{U, H}(B)=c_{S, H} \cdot B Q(\log B)+O_{\epsilon}\left(B^{10 / 11+\epsilon}\right),
$$

where $Q$ is a monic polynomial of degree 6 , and $c_{S, H}$ is the constant predicted by Peyre.

Proof. [BBD05, Theorem 1].

In fact, we prove a stronger statement than a meromorphic continuation of $Z_{U, H}(s)$ to $\Re e(s)>10 / 11$ :

TheOREm 7.5. For $S$ and $U$ as in Theorem 7.4. $(s-1)^{7} \cdot Z_{U, H}(s)$ has a holomorphic continuation to the halfplane $\Re e(s)>9 / 10$.

Proof. [BBD05, Theorem 2]. 



\section{CHAPTER 8}

\section{On a constant arising in Manin's conjecture}

\subsection{Introduction}

For $1 \leq r \leq 8$, let $S_{r}$ be a smooth Del Pezzo surface of degree $9-r$ (see Section 1.3). Suppose that $S_{r}$ is split over $\mathbb{Q}$.

An important object associated to $S_{r}$ is the effective cone $\Lambda_{\text {eff }}\left(S_{r}\right)$ (see Definition 1.8, i.e., the closed convex cone in

$$
\operatorname{Pic}\left(S_{r}\right)_{\mathbb{R}}:=\operatorname{Pic}\left(S_{r}\right) \otimes_{\mathbb{Z}} \mathbb{R}
$$

which is generated by the classes of effective divisors. For $r \geq 2, \Lambda_{\text {eff }}\left(S_{r}\right)$ is generated by the classes of the $(-1)$-curves by Lemma 1.15. As always, we identify $\operatorname{Pic}\left(S_{r}\right)_{\mathbb{R}}$ and its dual using the intersection form.

This chapter, whose results first appeared in Der06a, is concerned with the constant $\alpha\left(S_{r}\right)$ appearing in the leading constant of Manin's conjecture, cf. Section 7.2. Its definition is due to Peyre [Pey95, Definition 2.4]; see [PT01b, Section 6] for more details.

Definition 8.1. Let $\Lambda_{\text {eff }}\left(S_{r}\right)$ be the effective cone, $\Lambda_{\text {eff }}^{\vee}\left(S_{r}\right)$ its dual, and $-K_{S_{r}}$ the anticanonical class on $S_{r}$. Then we define

$$
\alpha\left(S_{r}\right):=\operatorname{Vol}\left(P_{r}\right)
$$

where

$$
P_{r}:=\left\{x \in \Lambda_{\text {eff }}^{\vee}\left(S_{r}\right) \mid\left(-K_{S_{r}}, x\right)=1\right\},
$$

and the Lebesgue measure on the hyperplane

$$
\left\{x \in \operatorname{Pic}\left(S_{r}\right)_{\mathbb{R}} \mid\left(-K_{S_{r}}, x\right)=1\right\}
$$

is defined by the $r$-form $\mathrm{d} \mathbf{x}$ such that $\mathrm{d} \mathbf{x} \wedge \mathrm{d} \omega=\mathrm{d} \mathbf{y}$, where $\mathrm{d} \mathbf{y}$ is the form corresponding to the natural Lebesgue measure on $\operatorname{Pic}\left(S_{r}\right)_{\mathbb{R}}$ and $\mathrm{d} \omega$ is the linear form defined by $-K_{S_{r}}$ on $\operatorname{Pic}\left(S_{r}\right)_{\mathbb{R}}$.

For small $r$, the calculation of $\alpha\left(S_{r}\right)$ can be carried out directly by hand (see [Bre02, Section 1.3] for the case $r=4$ ). For larger $r$, especially $r=8$, a direct calculation seems to be currently impossible even with the help of software like Polymake GJ00. In this case, the cone $\Lambda_{\text {eff }}\left(S_{8}\right)$ has 240 generators, while $\Lambda_{\text {eff }}^{\vee}\left(S_{8}\right)$ has 19440 generators. A direct calculation of $\alpha\left(S_{8}\right)$ would require a triangulation of $\Lambda_{\text {eff }}^{\vee}\left(S_{8}\right)$, which seems to be out of reach for today's software and hardware.

Therefore, we need a more detailed knowledge of $\Lambda_{\text {eff }}^{\vee}\left(S_{r}\right)$. See Section 1.4 for details on the action of the Weyl group $W_{r}$ on $\operatorname{Pic}\left(S_{r}\right)$. Our main result which will allow us to compute $\alpha\left(S_{r}\right)$ recursively is:

TheOREM 8.2. For $r \geq 3$, the nef cone $\Lambda_{\text {eff }}^{\vee}\left(S_{r}\right)$ has $N_{r}$ faces, where $N_{r}$ is the number of $(-1)$-curves on $S_{r}$. Each face is isomorphic to $\Lambda_{\mathrm{eff}}^{\vee}\left(S_{r-1}\right)$. 
The Weyl group $W_{r}$ acts transitively on the faces and fixes $-K_{S_{r}}$ in the interior of $\Lambda_{\mathrm{eff}}^{\vee}\left(S_{r}\right)$.

This observation is a crucial step in the proof of the following recursive formula for $\alpha\left(S_{r}\right)$; see Table 8.1 for the values of $\alpha\left(S_{r}\right)$ and $N_{r}$.

Theorem 8.3. Let $S_{r}$ be a split smooth Del Pezzo surface of degree $9-r$. We have $\alpha\left(S_{2}\right)=1 / 24$ and

$$
\alpha\left(S_{r}\right)=\frac{N_{r} \cdot \alpha\left(S_{r-1}\right)}{r \cdot(9-r)}
$$

for $3 \leq r \leq 8$. Furthermore, $\alpha\left(S_{1}\right)=1 / 6, \alpha\left(\mathbb{P}^{1} \times \mathbb{P}^{1}\right)=1 / 4$, and $\alpha\left(\mathbb{P}^{2}\right)=1$.

\begin{tabular}{|c||c|c|c|c|c|c|c|}
\hline$r$ & 2 & 3 & 4 & 5 & 6 & 7 & 8 \\
\hline \hline$N_{r}$ & 3 & 6 & 10 & 16 & 27 & 56 & 240 \\
\hline$\alpha\left(S_{r}\right)$ & $1 / 24$ & $1 / 72$ & $1 / 144$ & $1 / 180$ & $1 / 120$ & $1 / 30$ & 1 \\
\hline
\end{tabular}

TABle 8.1. Smooth Del Pezzo surfaces

Next, we consider split singular Del Pezzo surfaces $S_{r}^{\prime}$ (see Section 1.5) with minimal desingularizations $\widetilde{S}_{r}^{\prime}$ of degree $9-r$. The definition of $\alpha\left(\widetilde{S}_{r}^{\prime}\right)$ is almost the same as for smooth Del Pezzo surfaces: we must consider $-K_{\widetilde{S}_{r}}$, $\Lambda_{\text {eff }}\left(\widetilde{S}_{r}^{\prime}\right)$ and $\Lambda_{\text {eff }}^{\vee}\left(\widetilde{S}_{r}^{\prime}\right)$ in $\operatorname{Pic}\left(\widetilde{S}_{r}^{\prime}\right)$ of rank $r+1$. We will see in Proposition 8.11 that $\Lambda_{\text {eff }}\left(\widetilde{S}_{r}^{\prime}\right)$ is generated by the negative curves, including $(-2)$-curves, in this case.

As explained in Section 1.4, negative curves on $\widetilde{S}_{r}^{\prime}$ are related to the $(-1)$ curves on a smooth Del Pezzo surface $S_{r}$ of the same degree and certain root systems in $\operatorname{Pic}\left(S_{r}\right)_{\mathbb{R}}$.

More precisely, recall that, for $r \geq 2$, we can identify $\operatorname{Pic}\left(\widetilde{S}_{r}^{\prime}\right)$ and $\operatorname{Pic}\left(S_{r}\right)$ together with the intersection form and the anticanonical classes $-K_{\widetilde{S}_{r}^{\prime}}$ and $-K_{S_{r}}$. Then the $(-2)$-curves of $\widetilde{S}_{r}^{\prime}$ are the simple roots $\Delta$ of a root system $\Phi$ in $\operatorname{Pic}\left(S_{r}\right)_{\mathbb{R}}$, and the $(-1)$-curves of $\widetilde{S}_{r}^{\prime}$ are the $(-1)$-curves of $S_{r}$ which lie in the fundamental chamber $\mathcal{C}_{0}$ of $\Phi$.

The root system $\Phi$ is the direct sum of the irreducible root systems associated to the singularities of $S_{r}^{\prime}$. Let $W$ be the Weyl group associated to $\Phi$, generated by the reflections $s_{E}$ associated to the $(-2)$-curves $E$. The space $\operatorname{Pic}\left(S_{r}\right)_{\mathbb{R}}$ is the union of chambers $\mathcal{C}_{w}:=w\left(\mathcal{C}_{0}\right)$ for all $w \in W$, where the intersection of two chambers has lower dimension.

This description of the negative curves on $\widetilde{S}_{r}^{\prime}$ in terms of $(-1)$-curves on the smooth Del Pezzo surface $S_{r}$ of the same degree and the action of the Weyl group $W$ leads us to the following result on the structure of the nef cone $\Lambda_{\text {eff }}^{\vee}\left(\widetilde{S}_{r}^{\prime}\right)$ of $\widetilde{S}_{r}^{\prime}$ :

Theorem 8.4. For $r \geq 2$, we have

$$
\Lambda_{\text {eff }}^{\vee}\left(\widetilde{S}_{r}^{\prime}\right)=\Lambda_{\text {eff }}^{\vee}\left(S_{r}\right) \cap \mathcal{C}_{0} .
$$

Every $w \in W$ maps $\Lambda_{\mathrm{eff}}^{\vee}\left(\widetilde{S}_{r}^{\prime}\right)$ isomorphically to $\Lambda_{\mathrm{eff}}^{\vee}\left(S_{r}\right) \cap \mathcal{C}_{w}$.

It leads to the following result: 
Theorem 8.5. For $2 \leq r \leq 8$, let $S_{r}^{\prime}$ be a split singular Del Pezzo surface of degree $9-r$ with minimal desingularization $\widetilde{S}_{r}^{\prime}$, and let $S_{r}$ be a split smooth Del Pezzo surface of the same degree. Let $\Phi$ be the root system describing the singularities of $S_{r}^{\prime}$. Let $\# W$ be the order of the Weyl group $W$ associated to $\Phi$. Then

$$
\alpha\left(\widetilde{S}_{r}^{\prime}\right)=\alpha\left(S_{r}\right) / \# W
$$

where the values of $\alpha\left(S_{r}\right)$ can be found in Table 8.1. Furthermore, for the Hirzebruch surface $F_{2}$, we have $\alpha\left(F_{2}\right)=1 / 8$.

If $S_{r}^{\prime}$ has exactly one singularity, the root system is irreducible. The number $\# W$ of elements of the corresponding Weyl group can be found in Table 8.2. For more than one singularity, we must multiply the numbers corresponding to each of them.

\begin{tabular}{|c||c|c|c|c|c|}
\hline$\Phi$ & $\mathbf{A}_{n}$ & $\mathbf{D}_{n}$ & $\mathbf{E}_{6}$ & $\mathbf{E}_{7}$ & $\mathbf{E}_{8}$ \\
\hline \hline$\# W$ & $(n+1) !$ & $2^{n-1} \cdot n !$ & 51840 & 2903040 & 696729600 \\
\hline
\end{tabular}

TABLE 8.2. Weyl groups

For examples of the calculation of $\alpha\left(S_{r}\right)$ for non-split Del Pezzo surfaces, see Pey95, [PT01b, Section 6], [BF04], and [BB05].

\subsection{Smooth Del Pezzo surfaces}

Let $S_{r}$ be the blow-up of $\mathbb{P}^{2}$ in $2 \leq r \leq 8$ points in general position. By Lemma 1.15 , the effective cone $\Lambda_{\text {eff }}\left(S_{r}\right)$ is generated by the $(-1)$-curves, whose number is $N_{r}$ as listed in Table 8.1 .

Lemma 8.6. Let $E$ be a (-1)-curve on $S_{r}$ for $r \geq 3$. If $D \in \operatorname{Pic}\left(S_{r}\right)$ fulfills $(D, E)=0$ and $\left(D, E^{\prime}\right) \geq 0$ for all $(-1)$-curves $E^{\prime}$ such that $\left(E, E^{\prime}\right)=$ 0 , then $D$ is nef.

Proof. As the (-1)-curves generate the effective cone (Lemma 1.15), we must show that $\left(D, E^{\prime}\right) \geq 0$ also holds for all $(-1)$-curves, regardless of the value of $\left(E, E^{\prime}\right)$.

If $\left(E, E^{\prime}\right)<0$, then $E^{\prime}=E$, and $(D, E)=0$. If $\left(E, E^{\prime}\right)=0$, then $\left(D, E^{\prime}\right) \geq 0$ by assumption.

We proceed by induction on $\left(E, E^{\prime}\right)$. If $\left(E, E^{\prime}\right)=n \geq 1$, then $E+E^{\prime}$ is an (n)-ruling as in Definition 3.1. (Note that $n=2$ occurs only for $r \in\{7,8\}$, and $n=3$ only for $r=8$; furthermore, $n \geq 4$ is impossible.)

Any $(n)$-ruling can be written in at least two ways as the sum of two negative curves (see Section 3.2 for $n=1$, and Section 3.4 and Section 3.5 for $n \in\{2,3\})$, say $E+E^{\prime}=E_{1}+E_{2}$, where $E \notin\left\{E_{1}, E_{2}\right\}$. Then and

$$
\left(E, E_{1}\right)+\left(E, E_{2}\right)=\left(E, E^{\prime}\right)+(E, E)=n-1,
$$

where $\left(E, E_{1}\right)$ and $\left(E, E_{2}\right)$ are both non-negative. Therefore, the induction hypothesis holds for $E_{1}, E_{2}$, and

$$
\left(D, E^{\prime}\right)=\left(D, E+E^{\prime}\right)=\left(D, E_{1}+E_{2}\right)=\left(D, E_{1}\right)+\left(D, E_{2}\right) \geq 0
$$

completes the induction. 
Proof of Theorem 8.2. By definition, $\Lambda_{\text {eff }}^{\vee}\left(S_{r}\right)$ is the intersection of the closed halfspaces $E^{\geq 0}$ (see Section 1.4) for all generators $E$ of $\Lambda_{\text {eff }}\left(S_{r}\right)$, which are exactly the $(-1)$-curves by Lemma 1.15, By [FM02, Lemma 5.3], $W_{r}$ acts transitively on the $(-1)$-curves. This symmetry implies that each $(-1)$ curve $E$ defines a proper face $F_{E}:=\Lambda_{\text {eff }}\left(S_{r}\right) \cap E^{=0}$, and that $W_{r}$ acts transitively on the faces $F_{E}$.

Consider $S_{r}$ as the blow-up of $S_{r-1}$ in one point, resulting in the exceptional divisor $E_{r}$. Then

$$
\operatorname{Pic}\left(S_{r}\right)=\operatorname{Pic}\left(S_{r-1}\right) \oplus \mathbb{Z} \cdot E_{r}
$$

is an orthogonal sum.

We claim that $F_{E_{r}}=\Lambda_{\text {eff }}\left(S_{r-1}\right)$, where we regard $\Lambda_{\text {eff }}\left(S_{r-1}\right) \subset \operatorname{Pic}\left(S_{r-1}\right)$ as embedded into $\operatorname{Pic}\left(S_{r}\right)$.

Indeed, if $D \in \Lambda_{\text {eff }}\left(S_{r-1}\right)$, then $\left(D, E_{r}\right)=0$, and $(D, E) \geq 0$ for all $(-1)$ curves $E$ of $S_{r-1}$, which are exactly the $(-1)$-curves of $S_{r}$ with $\left(E, E_{r}\right)=0$. By Lemma 8.6, we have $(D, E) \geq 0$ for all $(-1)$-curves of $S_{r}$.

On the other hand, if $D \in E_{r}^{=0}$, then $D \in \operatorname{Pic}\left(S_{r-1}\right)$. If $D \in \Lambda_{\text {eff }}\left(S_{r}\right)$, then $(D, E) \geq 0$ for all $(-1)$-curves of $S_{r}$, which includes the (-1)-curves of $S_{r-1}$, proving the other direction.

The root system corresponding to $W_{r}$ is

$$
\Phi_{r}=\left\{D \in \operatorname{Pic}\left(S_{r}\right) \mid(D, D)=-2,\left(D,-K_{S_{r}}\right)=0\right\} .
$$

Since $W_{r}$ is generated by the reflections $E \mapsto E+(D, E) \cdot D$ corresponding to the roots $D \in \Phi_{r}$, the anticanonical class $-K_{S_{r}}$ is fixed under $W_{r}$. This completes the proof of Theorem 8.2 .

Proof of Theorem 8.3. The polytope $P_{r}$ is the intersection of the $N_{r}$ half-spaces $E^{\geq 0}$ (where $E$ runs through the $(-1)$-curves of $S_{r}$ ) in the $r$ dimensional space $-K_{S_{r}}=0$.

Note that $\left(-K_{S_{r}},-K_{S_{r}}\right)=9-r$. Consequently, $Q:=\frac{1}{9-r}\left(-K_{S_{r}}\right) \in$ $-K_{S_{r}}=1$, and since $\left(-K_{S_{r}}, E\right)=1$ for any $(-1)$-curve $E$, the point $Q$ is in the interior of $P_{r}$.

Consider the convex hull $P_{E}$ of $Q$ and of the face $P_{r} \cap E^{=0}$ of $P_{r}$ corresponding to $E$. Then $P_{r}$ is the union of the $P_{E}$ for all $(-1)$-curves $E$, and since their intersections are lower-dimensional,

$$
\operatorname{Vol}\left(P_{r}\right)=\sum_{E} \operatorname{Vol}\left(P_{E}\right)
$$

As the intersection form and $-K_{S_{r}}$ are invariant under $W_{r}$, the Weyl group $W_{r}$ acts on $-K_{S_{r}}^{=1}$ and, therefore, on $P_{r}$. As in Theorem 8.2, it permutes the faces of $P_{r}$ transitively. As $Q$ is fixed under $W_{r}$ and the volume is invariant under $W_{r}$, we have $\operatorname{Vol}\left(P_{r}\right)=N_{r} \cdot \operatorname{Vol}\left(P_{E}\right)$ for any $(-1)$-curve E.

As in the proof of Theorem 8.2, we consider $S_{r}$ as the blow-up of $S_{r-1}$ in one point, resulting in the exceptional divisor $E_{r}$, with the orthogonal sum

$$
\operatorname{Pic}\left(S_{r}\right)=\operatorname{Pic}\left(S_{r-1}\right) \oplus \mathbb{Z} \cdot E_{r} .
$$

We claim that $P_{r} \cap E_{r}^{=0}=P_{r-1}$. In view of Theorem 8.2, it remains to prove that $\left(D,-K_{S_{r}-1}\right)=1$ is equivalent to $\left(D,-K_{S_{r}}\right)=1$ on $E_{r}^{=0}$. This follows directly from $-K_{S_{r}}=-K_{S_{r}-1}-E_{r}$. 
Therefore, $P_{E_{r}}$ is a cone over the $(r-1)$-dimensional polytope $P_{r-1}$ in the $r$-dimensional space $-K_{\bar{S}_{r}}$. A cone of height 1 over $P_{r-1}$ has volume $\operatorname{Vol}\left(P_{r-1}\right) / r$. As $E_{r}$ is orthonormal to $\operatorname{Pic}\left(S_{r-1}\right)$, and $\left(-K_{S_{r}}, E_{r}\right)=1$, the distance of $Q$ to $P_{r-1}$ is $1 /(9-r)$. Hence,

$$
\operatorname{Vol}\left(P_{E_{r}}\right)=\frac{\operatorname{Vol}\left(P_{r-1}\right)}{r \cdot(9-r)}
$$

Together with $\alpha\left(S_{r-1}\right)=\operatorname{Vol}\left(P_{r-1}\right)$ and $\operatorname{Vol}(P)=N_{r} \cdot \operatorname{Vol}\left(P_{E_{r}}\right)$, this completes the proof of the recursive formula.

For $r=2$, we have $\Lambda_{\text {eff }}=\left\langle E_{1}, E_{2}, H-E_{1}-E_{2}\right\rangle$ and $-K_{S_{2}}=3 H-E_{1}-$ $E_{2}$. Consequently, $\alpha\left(S_{2}\right)$ is the volume of

$$
\begin{aligned}
& \left\{\left(a_{0}, a_{1}, a_{2}\right) \in \mathbb{R}^{3} \mid 3 a_{0}-a_{1}-a_{2}=1, a_{1} \geq 0, a_{2} \geq 0, a_{0}-a_{1}-a_{2} \geq 0\right\} \\
= & \left\{\left(a_{0}, a_{1}\right) \in \mathbb{R}^{2} \mid a_{1} \geq 0,3 a_{0}-a_{1}-1 \geq 0,-2 a_{0}+1 \geq 0\right\} \\
= & \text { convex hull of }(1 / 3,0),(1 / 2,0),(1 / 2,1 / 2),
\end{aligned}
$$

which is a rectangular triangle whose legs have length $1 / 6$ and $1 / 2$. Hence, $\alpha\left(S_{2}\right)=1 / 24$, while $\alpha\left(S_{1}\right)=1 / 6, \alpha\left(\mathbb{P}^{1} \times \mathbb{P}^{1}\right)=1 / 4$, and $\alpha\left(\mathbb{P}^{2}\right)=1$ can also be calculated directly, which completes the proof of Theorem 8.3

Remark 8.7. By the proof of [Pey95, Lemme 9.4.2], $\alpha\left(S_{1}\right)=1 / 6$, and by the proof of Pey95, Lemme 10.4.2],

$$
\alpha\left(S_{2}\right)=1 / 3 \cdot \operatorname{Vol}\left\{\left(x_{1}, x_{2}\right) \in \mathbb{R}_{>0}^{2} \mid x_{1}+x_{2} \leq 1 / 2\right\},
$$

which is clearly $1 / 24$ and, therefore, agrees with our result. Note that the recursion formula does not hold for $r=2$ :

$$
\alpha\left(S_{2}\right)=\frac{1}{24} \neq \frac{N_{2} \cdot \alpha\left(S_{1}\right)}{2 \cdot(9-2)}=\frac{1}{28} .
$$

The value $\alpha\left(S_{4}\right)=1 /(6 \cdot 4 !)$ was previously calculated in Bre02, Section 1.3].

\subsection{Singular Del Pezzo surfaces}

We prove Theorem 8.4 and Theorem 8.5. For $r \geq 2$, let $S^{\prime}:=\widetilde{S}_{r}^{\prime}$ be a generalized Del Pezzo surface of degree $9-r$, and let $S:=S_{r}$ be a smooth Del Pezzo surface of the same degree. Identify $\operatorname{Pic}(S)$ and $\operatorname{Pic}\left(S^{\prime}\right)$ together with the intersection form as in the introduction.

Lemma 8.8. Let $L$ be a (-1)-curve on $S$. Then there is a (-1)-curve $L_{0}$ on $S^{\prime}$ and $w \in W$ such that $L=w\left(L_{0}\right)$.

Proof. The $(-2)$-curves form a system $\Delta$ of simple roots in $\operatorname{Pic}(S)_{\mathbb{R}}$. By the theory of root systems, the fundamental chamber $\mathcal{C}_{0}$ is a fundamental domain for the action of $W$, so we can find a $w \in W$ and $x \in \mathcal{C}_{0}$ such that $L=w(x)$. As $W$ acts on the $(-1)$-curves on $S$, we conclude that $x$ is a $(-1)$-curve on $S^{\prime}$.

Lemma 8.9. Let $L=w\left(L_{0}\right)$ as in Lemma 8.8, Then there are positive roots $E_{1}, \ldots, E_{k}$ which fulfill the following conditions: We have $w=w_{k}$, and $\left(L_{i-1}, E_{i}\right) \geq 0$ for $i \in\{1, \ldots, k\}$, where $w_{i}:=s_{E_{i}} \circ \cdots \circ s_{E_{1}}$ and $L_{i}:=w_{i}\left(L_{0}\right)$. 
Proof. Consider a minimal gallery $\mathcal{C}_{0}, \mathcal{C}_{1}, \ldots, \mathcal{C}_{k}$ from $\mathcal{C}_{0}$ to $\mathcal{C}_{k}:=w\left(\mathcal{C}_{0}\right)$ (cf. [Bro89, Section II.1]). Here, $k$ is the length of $w$, and for $i \in\{1, \ldots, k\}$, there is a root $E_{i}$ such that $\mathcal{C}_{i}=s_{E_{i}}\left(\mathcal{C}_{i-1}\right)$, and $E_{i}^{=0}$ is the wall between $\mathcal{C}_{i-1}$ and $\mathcal{C}_{i}$. As $s_{E_{i}}=s_{-E_{i}}$, we may assume that $E_{1}, \ldots, E_{k}$ are positive roots. We have $\mathcal{C}_{i}=w_{i}\left(\mathcal{C}_{0}\right)$ and $w=w_{k}$.

For $x \in \mathcal{C}_{0}$, we have $\left(w_{i-1}(x), E_{i}\right) \geq 0$ and $\left(w_{i}(x), E_{i}\right) \leq 0$, while for all roots $E \neq \pm E_{i}$, the numbers $\left(w_{i-1}(x), E\right)$ and $\left(w_{i}(x), E\right)$ have the same sign (or are both zero).

As $L_{0} \in \mathcal{C}_{0}$ and $L_{i}=w_{i}\left(L_{0}\right)$, we have $\left(L_{i-1}, E_{i}\right) \geq 0$.

Let $C$ be the convex cone in $\operatorname{Pic}\left(S^{\prime}\right) \cong \operatorname{Pic}(S)$ generated by the negative curves on $S^{\prime}$, and let $C^{\vee}$ be its dual with respect to the intersection form. Recall from Lemma 1.15 that $\Lambda_{\text {eff }}(S)$ is generated by the $(-1)$-curves on $S$ since $r \geq 2$.

Lemma 8.10. Let $x$ be in $C^{\vee}$. Then $x \in \Lambda_{\text {eff }}^{\vee}(S)$.

Proof. Let $x \in C^{\vee}$, i.e., $(x, E) \geq 0$ for all negative curves $E$ on $S^{\prime}$. We must show that $(x, L) \geq 0$ for all $(-1)$-curves $L$ on $S$.

Let $L$ be a $(-1)$-curve on $S$, and $w, k, w_{i}, L_{i}, E_{i}$ as in Lemma 8.9. We use induction on the length $k$ of $w$. If $k=0$, then $L=L_{0}$ is a $(-1)$-curve on $S^{\prime}$, so $(x, L) \geq 0$ by assumption.

If $k>0$, then $L=s_{E_{k}}\left(L_{k-1}\right)$. Then

$$
\begin{aligned}
(x, L)=\left(x, s_{E_{k}}\left(L_{k-1}\right)\right) & =\left(x, L_{k-1}+\left(L_{k-1}, E_{k}\right) \cdot E_{k}\right) \\
& =\left(x, L_{k-1}\right)+\left(L_{k-1}, E_{k}\right) \cdot\left(x, E_{k}\right) .
\end{aligned}
$$

As $L_{k-1}=w_{k-1}\left(L_{0}\right)$ for $w_{k-1}:=s_{E_{k-1}} \circ \cdots \circ s_{E_{1}}$ which has length $k-1$, we have $\left(x, L_{k-1}\right) \geq 0$ by induction. Since the positive root $E_{k}$ is a nonnegative linear combination of the simple roots, which are exactly the $(-2)$ curves on $S^{\prime}$, we have $\left(x, E_{k}\right) \geq 0$. Furthermore, $\left(L_{k-1}, E_{k}\right) \geq 0$ because of Lemma 8.9 ,

Proposition 8.11. The effective cone $\Lambda_{\mathrm{eff}}\left(S^{\prime}\right)$ is generated by the negative curves on $S^{\prime}$.

Proof. It is easy to check for each $r \in\{2, \ldots, 8\}$ that $\Lambda_{\text {eff }}^{\vee}(S)$ is contained in $\Lambda_{\text {eff }}(S)$, which is generated by the negative curves on $S$.

By Lemma 8.10, we have $C^{\vee} \subset \Lambda_{\text {eff }}^{\vee}(S)$. Taking duals again, which reverses the inclusion, we conclude $\Lambda_{\text {eff }}(S) \subset C$. Together, we have

$$
C^{\vee} \subset \Lambda_{\text {eff }}^{\vee}(S) \subset \Lambda_{\text {eff }}(S) \subset C \text {. }
$$

Every prime divisor on $S^{\prime}$ is either a negative curve or has non-negative intersection number with all negative curves on $S^{\prime}$. Therefore, it is either contained in $C$ or in $C^{\vee} \subset C$. Hence, $C=\Lambda_{\text {eff }}\left(S^{\prime}\right)$.

Proof of Theorem 8.4. We claim that $\Lambda_{\text {eff }}^{\vee}\left(S^{\prime}\right)=\Lambda_{\text {eff }}^{\vee}(S) \cap \mathcal{C}_{0}$. On the one hand, every $x \in \widehat{\Lambda}_{\text {eff }}\left(S^{\prime}\right)$ is in $\mathcal{C}_{0}$ by definition, and $x \in \Lambda_{\text {eff }}^{\vee}(S)$ by Lemma 8.10. On the other hand, if $x \in \mathcal{C}_{0}$, then $(x, E) \geq 0$ for all $(-2)$ curves on $S^{\prime}$, and since the $(-1)$-curves on $S^{\prime}$ are a subset of the $(-1)$-curves on $S$ (according to the identifications of the Picard groups that we have made), the condition $x \in \Lambda_{\text {eff }}^{\vee}(S)$ implies $(x, L) \geq 0$ for all $(-1)$-curves on $S^{\prime}$, so $x \in \Lambda_{\text {eff }}^{\vee}(S) \cap \mathcal{C}_{0}$ implies $x \in \Lambda_{\text {eff }}^{\vee}\left(S^{\prime}\right)$. 
As $W$ acts on the $(-1)$-curves on $S$, any $w \in W$ maps $\Lambda_{\text {eff }}^{\vee}(S) \cap \mathcal{C}_{0}$ isomorphically to $\Lambda_{\text {eff }}^{\vee}(S) \cap w\left(\mathcal{C}_{0}\right)$. This completes the proof.

Proof of Theorem 8.5. If $x \in-K_{\bar{S}}^{=1}$, then for any (-2)-curve $E$ on $S^{\prime}$, $\left(s_{E}(x),-K_{S}\right)=\left(x+(x, E) \cdot E,-K_{S}\right)=\left(x,-K_{S}\right)+(x, E) \cdot\left(E,-K_{S}\right)=1$ since $\left(E,-K_{S}\right)=0$. Therefore, every $w \in W$ maps $-K_{\bar{S}}^{=1} \subset \operatorname{Pic}(S)_{\mathbb{R}}$ isomorphically to itself.

Together with Theorem 8.4 , this shows that $\Lambda_{\text {eff }}^{\vee}(S) \cap\left(-K_{\bar{S}}^{=1}\right)$ is the union of the sets

$$
V_{w}:=\Lambda_{\text {eff }}^{\vee}(S) \cap \mathcal{C}_{w} \cap\left(-K_{\bar{S}}^{=1}\right)
$$

for all $w \in W$, where the intersection of any two of these sets has lower dimension. Each $V_{w}$ is the isomorphic image of $V_{0}:=\Lambda_{\text {eff }}^{\vee}\left(S^{\prime}\right) \cap\left(-K_{S}^{=1}\right)$ under $w \in W$, and this isomorphism respects the measure on $-K_{S}^{\overline{1}}$. Hence, the volume of each $V_{w}$ is the volume of $V_{0}$. As the anticanonical class $-K_{S}$ on $S$ is identified with the anticanonical class $-K_{S^{\prime}}$ on $S^{\prime}$ under our identification of the Picard groups, we have $\alpha\left(\widetilde{S}^{\prime}\right)=\operatorname{Vol}\left(V_{0}\right)$, and as $\operatorname{Vol}(S) \cap$ $\left(-K_{\bar{S}}^{=1}\right)=\alpha(S)$, this shows $\# W \cdot \alpha\left(\widetilde{S}^{\prime}\right)=\alpha(S)$.

We calculate $\alpha\left(F_{2}\right)=1 / 8$ for the Hirzebruch surface $F_{2}$ directly, completing the proof of Theorem 8.5 . 



\section{CHAPTER 9}

\section{Manin's conjecture for a singular cubic surface}

\subsection{Introduction}

We prove Manin's conjecture (Conjecture 7.1) in the case of a cubic surface with a singularity of type $\mathbf{E}_{6}$ (see Example 1.27):

$$
S=\left\{\mathbf{x}=\left(x_{0}: x_{1}: x_{2}: x_{3}\right) \in \mathbb{P}^{3} \mid f(\mathbf{x})=x_{1} x_{2}^{2}+x_{2} x_{0}^{2}+x_{3}^{3}=0\right\}
$$

In Figure 9.1, we see points of height $\leq 1000$ on $S$.

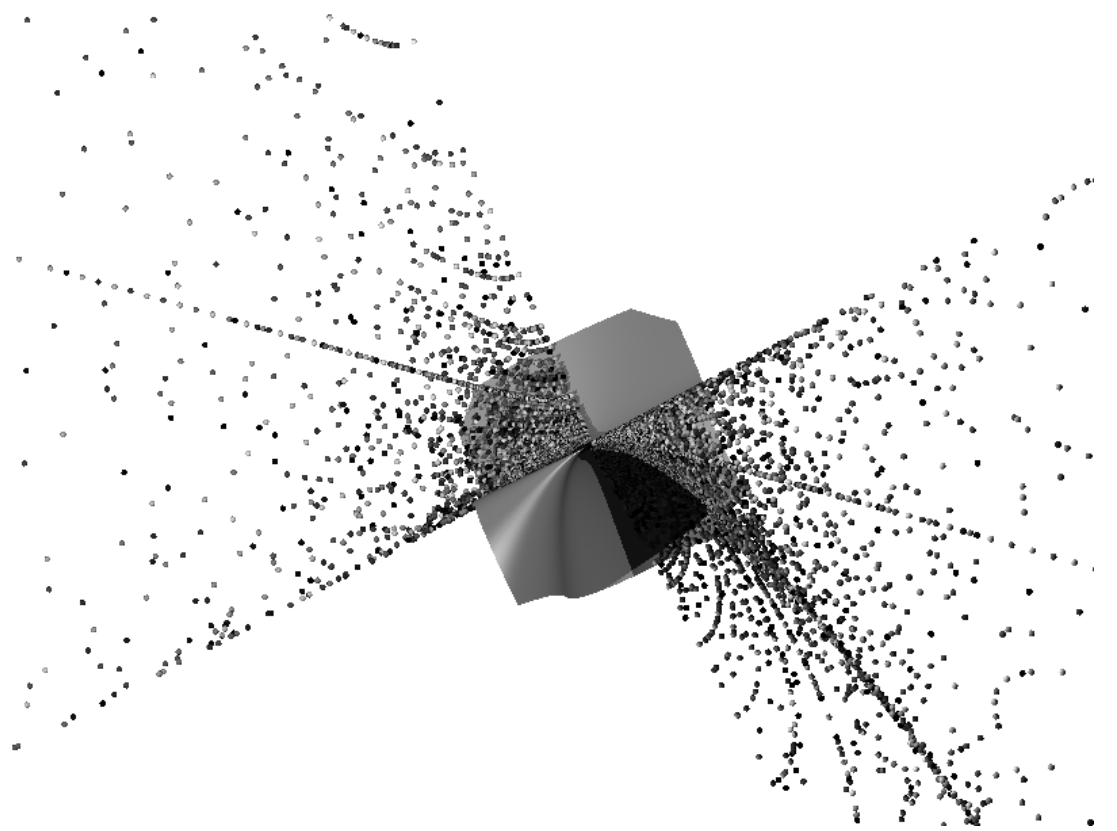

Figure 9.1. Points of height $\leq 1000$ on the $\mathbf{E}_{6}$ singular cubic surface $S$ with $x_{0}, x_{2}>0$.

THEOREM 9.1. Let $S$ be the cubic surface as above with a singularity of type $\mathbf{E}_{6}$ and let $H$ be the anticanonical height. Let $U:=S \backslash \ell$, where $\ell=\left\{x_{2}=x_{3}=0\right\}$ is the unique line on $S$.

Then

$$
N_{U, H}(B)=c_{S, H} B Q(\log B)+O\left(B(\log B)^{2}\right),
$$

where $Q$ is a monic polynomial of degree 6 , and the leading constant $c_{S, H}$ is the one conjectured by Peyre Pey95.

The invariants appearing in Manin's conjecture and Peyre's constant $c_{S, H}$ are calculated in Section 9.2 . 
The proof follows the strategy of de la Bretèche and Browning BB04 and uses the universal torsor. For our particular surface, the universal torsor has been calculated by Hassett and Tschinkel using the Cox ring; the torsor is a hypersurface in the 10-dimensional affine space [HT04. In addition to the equation defining the torsor we need to derive certain coprimality conditions between the coordinates. In Section 9.3 we compute the torsor and determine these conditions following the more direct approach of HeathBrown, Browning and de la Bretèche [HB03, [Bro04, [BB04].

The next step is to count the number of integral points on the universal torsor and satisfying the coprimality conditions and subject to certain bounds, given by lifting the height function to the torsor. For three of the ten variables on the torsor, this summation is done in Section 9.5 by elementary methods from analytic number theory. The summation over the last seven variables, completing the proof of Theorem 9.1, is carried out in Section 9.6 .

The results of this chapter first appeared in Der05. Section 7.3 discusses joint work with de la Bretèche and Browning which proves a version of Theorem 9.1 with an improved error term, to appear in [BBD05.

\subsection{Manin's conjecture}

In this section, we calculate the invariants appearing in Manin's conjecture and its refinement by Peyre. We use the notation from Pey95.

Lemma 9.2. Manin's conjecture predicts in case of $S$ as defined in 9.1):

$$
N_{U, H}(B) \sim c_{S, H} B(\log B)^{6},
$$

where $c_{S, H}=\alpha(\widetilde{S}) \beta(\widetilde{S}) \omega_{H}(\widetilde{S})$ with

$$
\begin{aligned}
\alpha(\widetilde{S}) & :=\frac{1}{6 ! \prod_{i} \lambda_{i}}=\frac{1}{6 ! \cdot 2 \cdot 3 \cdot 4 \cdot 3 \cdot 4 \cdot 5 \cdot 6}=\frac{1}{6220800}, \\
\beta(\widetilde{S}) & :=1, \\
\omega_{H}(\widetilde{S}) & :=\omega_{\infty} \cdot \omega_{0},
\end{aligned}
$$

and

$$
\begin{aligned}
\lambda & =\left(\lambda_{1}, \lambda_{2}, \lambda_{3}, \lambda_{\ell}, \lambda_{4}, \lambda_{5}, \lambda_{6}\right):=(2,3,4,3,4,5,6), \\
\omega_{\infty} & \left.:=6 \iiint_{\left\{(t, u, v) \in \mathbb{R}^{3} \mid\right.} 1 t v^{3}|\leq 1,| t^{2}+u^{3}|\leq 1,0 \leq v \leq 1,| u v^{4} \mid \leq 1\right\} \\
\omega_{0} & :=\prod_{p}\left(1-\frac{1}{p}\right)^{7}\left(1+\frac{7}{p}+\frac{1}{p^{2}}\right) .
\end{aligned}
$$

Proof. By Theorem 8.5, $\alpha(\widetilde{S})=\alpha^{\prime} / \# W$, where $\alpha^{\prime}=1 / 120$ is the constant associated to smooth split cubic surfaces, and $\# W=51840$ is the order of the Weyl group $W$ associated to the root system $\mathbf{E}_{6}$.

The surface $S$ is split over $\mathbb{Q}$, so that

$$
\beta(\widetilde{S})=\# H^{1}(\mathbb{Q}, \operatorname{Pic}(\widetilde{S}))=1
$$


By definition,

$$
\omega_{H}(\widetilde{S})=\lim _{s \rightarrow 1}\left((s-1)^{\mathrm{rk} \operatorname{Pic}(\widetilde{S})} L(s, \operatorname{Pic}(\widetilde{S}))\right) \omega_{\infty}(\widetilde{S}) \prod_{p} \frac{\omega_{p}(\widetilde{S})}{L_{p}(1, \operatorname{Pic}(\widetilde{S}))},
$$

where, in our case,

$$
\lim _{s \rightarrow 1}\left((s-1)^{\mathrm{rk} \operatorname{Pic}(\widetilde{S})} L(s, \operatorname{Pic}(\widetilde{S}))\right)=\lim _{s \rightarrow 1}\left((s-1)^{7} \zeta(s)^{7}\right)=1
$$

and

$$
L_{p}(1, \operatorname{Pic}(\widetilde{S}))^{-1}=(1-1 / p)^{7} .
$$

We use Peyre's method Pey95 to compute $\omega_{\infty}(\widetilde{S})$ and parameterize the points by writing $x_{1}$ as a function of $x_{0}, x_{2}, x_{3}$. Since $\mathbf{x}=-\mathbf{x}$ in $\mathbb{P}^{3}$, we may assume $x_{2} \geq 0$. Since $\frac{\mathrm{d}}{\mathrm{d} x_{1}} f=x_{2}^{2}$, the Leray form $\omega_{L}(\widetilde{S})$ is given by $x_{2}^{-2} \mathrm{~d} x_{0} \mathrm{~d} x_{3} \mathrm{~d} x_{2}$, and we obtain $\omega_{\infty}(\widetilde{S})$ from

$$
\iiint_{\left\{\left|x_{0}\right| \leq 1,\left|x_{2}^{-2}\left(x_{2} x_{0}^{2}+x_{3}^{3}\right)\right| \leq 1,0 \leq x_{2} \leq 1,\left|x_{3}\right| \leq 1\right\}} x_{2}^{-2} \mathrm{~d} x_{0} \mathrm{~d} x_{3} \mathrm{~d} x_{2},
$$

using the transformations

$$
x_{0}=t x_{2}^{1 / 2}, \quad x_{3}=u x_{2}^{2 / 3}, \quad x_{2}=v^{6} .
$$

The calculation of

$$
\omega_{p}(\widetilde{S})=1+\frac{7}{p}+\frac{1}{p^{2}}
$$

is done as in [BB04, Lemma 1], and we omit it here.

\subsection{The universal torsor}

The universal torsor

$$
\mathcal{T}_{\widetilde{S}}=\operatorname{Spec}\left(\mathbb{Q}\left[\xi_{1}, \xi_{2}, \xi_{3}, \xi_{\ell}, \xi_{4}, \xi_{5}, \xi_{6}, \tau_{1}, \tau_{2}, \tau_{\ell}\right] /\left(T\left(\xi_{i}, \tau_{i}\right)\right)\right)
$$

is given by the equation

$$
T\left(\xi_{i}, \tau_{i}\right)=\tau_{\ell} \xi_{\ell}^{3} \xi_{4}^{2} \xi_{5}+\tau_{2}^{2} \xi_{2}+\tau_{1}^{3} \xi_{1}^{2} \xi_{3}=0
$$

and the map $\Psi: \mathcal{T}_{\widetilde{S}} \rightarrow S=\operatorname{Spec}\left(\mathbb{Q}\left[x_{0}, \ldots, x_{3}\right] /(f(\mathbf{x}))\right)$ defined by

$$
\begin{aligned}
& \Psi^{*}\left(x_{0}\right)=\xi^{(1,2,2,0,1,2,3)} \tau_{2} \\
& \Psi^{*}\left(x_{1}\right)=\tau_{\ell} \\
& \Psi^{*}\left(x_{2}\right)=\xi^{(2,3,4,3,4,5,6)} \\
& \Psi^{*}\left(x_{3}\right)=\xi^{(2,2,3,1,2,3,4)} \tau_{1}
\end{aligned}
$$

where we use the notation $\xi^{\left(n_{1}, n_{2}, n_{3}, n_{\ell}, n_{4}, n_{5}, n_{6}\right)}:=\xi_{1}^{n_{1}} \xi_{2}^{n_{2}} \xi_{3}^{n_{3}} \xi_{\ell}^{n_{\ell}} \xi_{4}^{n_{4}} \xi_{5}^{n_{5}} \xi_{6}^{n_{6}}$. Note that $\Psi^{*}\left(x_{2}\right)=\xi^{\lambda}$ with $\lambda \in \mathbb{Z}^{7}$ as in Lemma 9.2 .

We establish a bijection between rational points on the surface $S$ and integral points on the torsor $\mathcal{T}_{\widetilde{S}}$ which are subject to certain coprimality conditions. More precisely, the coprimality conditions can be summarized in the following table, where a "-" means that the two variables are coprime, and a " $\times$ " that they may have common factors. For a variable combined with itself, "-" means that each prime occurs at most once (which can be 
expressed using the Möbius function $\mu$ as $|\mu(\cdot)|=1$ ), and " $\times$ " means that it may occur more often.

\begin{tabular}{c|ccccccc|ccc} 
& $\xi_{1}$ & $\xi_{2}$ & $\xi_{3}$ & $\xi_{\ell}$ & $\xi_{4}$ & $\xi_{5}$ & $\xi_{6}$ & $\tau_{1}$ & $\tau_{2}$ & $\tau_{\ell}$ \\
\hline$\xi_{1}$ & $\times$ & - & $\times$ & - & - & - & $\times$ & $\times$ & - & - \\
$\xi_{2}$ & - & - & - & - & - & - & $\times$ & - & $\times$ & - \\
$\xi_{3}$ & $\times$ & - & - & - & - & - & $\times$ & - & - & - \\
$\xi_{\ell}$ & - & - & - & $\times$ & $\times$ & $\times$ & $\times$ & - & - & $\times$ \\
$\xi_{4}$ & - & - & - & $\times$ & - & - & $\times$ & - & - & - \\
$\xi_{5}$ & - & - & - & $\times$ & - & - & $\times$ & - & - & - \\
$\xi_{6}$ & $\times$ & $\times$ & $\times$ & $\times$ & $\times$ & $\times$ & $\times$ & - & $\times$ & - \\
\hline$\tau_{1}$ & $\times$ & - & - & - & - & - & - & $\times$ & $\times$ & $\times$ \\
$\tau_{2}$ & - & $\times$ & - & - & - & - & $\times$ & $\times$ & $\times$ & $\times$ \\
$\tau_{\ell}$ & - & - & - & $\times$ & - & - & - & $\times$ & $\times$ & $\times$
\end{tabular}

We will refer to the

(9.4) coprimality conditions between $\xi_{1}, \ldots, \xi_{6}$ as given in the table.

Because of the torsor equation $T$, we can write the coprimality conditions for $\tau_{i}$ equivalently as

$$
\operatorname{gcd}\left(\tau_{1}, \xi_{2} \xi_{3} \xi_{\ell} \xi_{4} \xi_{5} \xi_{6}\right)=1
$$

and

$$
\operatorname{gcd}\left(\tau_{2}, \xi_{1} \xi_{3}\right)=1, \quad \operatorname{gcd}\left(\tau_{\ell}, \xi_{4} \xi_{5} \xi_{6}\right)=1 .
$$

Proposition 9.3. The map $\Psi$ induces a bijection between

$$
\mathcal{T}_{1}:=\left\{\left(\xi_{i}, \tau_{i}\right) \in \mathcal{T}_{\widetilde{S}}(\mathbb{Z}) \mid 9.4 \text {, 9.5, 9.6 hold, } \xi_{i}>0\right\}
$$

and $U(\mathbb{Q}) \subset S(\mathbb{Q})$.

The proof splits into two parts. First, we establish a similar bijection with slightly different coprimality conditions:

Lemma 9.4. Let $\mathcal{T}_{2}$ be set of all $\left(\xi_{i}, \tau_{i}\right) \in \mathcal{T}_{\widetilde{S}}(\mathbb{Z})$ such that $\xi_{i}>0$ and the coprimality conditions described by the table hold, except that the conditions

$$
\operatorname{gcd}\left(\xi_{3}, \tau_{1}\right)=1 \text { and } \operatorname{gcd}\left(\xi_{6}, \tau_{1}\right)=1
$$

in the table are replaced by

$$
\left|\mu\left(\xi_{1}\right)\right|=1 \text { and } \operatorname{gcd}\left(\xi_{1}, \xi_{3}\right)=1 .
$$

Then the map $\Psi$ induces a bijection between $\mathcal{T}_{2}$ and $U(\mathbb{Q}) \subset S(\mathbb{Q})$.

Proof. Using the method of [BB04, we show that the coprimality conditions lead to a bijection. We go through a series of coprimality considerations and replace the original variables by products of new ones which fulfill certain conditions. When doing this, the new variables will be uniquely determined.

Since $\mathbf{x}=-\mathbf{x}$, and $x_{2}=0$ is equivalent to $\mathbf{x} \in \ell$, we can write each $\mathbf{x} \in U(\mathbb{Q})$ uniquely such that $x_{i} \in \mathbb{Z}, x_{2}>0$, and $\operatorname{gcd}\left(x_{i}\right)=1$.

- Note that $x_{2} \mid x_{3}^{3}$. Write $x_{2}=y_{1} y_{2}^{2} y_{3}^{3}$ with $y_{i} \in \mathbb{Z}_{>0}$, where each triple occurrence of a prime factor of $x_{3}$ is put in $y_{3}$ and each double occurrence in $y_{2}$, so that $y_{1}, y_{2}, y_{3}$ are unique if we assume 
$\left|\mu\left(y_{1} y_{2}\right)\right|=1$. Then $x_{3}=y_{1} y_{2} y_{3} z$ must hold for a suitable $z \in \mathbb{Z}$. Substituting into $f$ and dividing by $y_{1} y_{2}^{2} y_{3}^{3}$ gives

$$
f_{1}\left(x_{0}, x_{1}, y_{1}, y_{2}, y_{3}, z\right)=x_{1} y_{1} y_{2}^{2} y_{3}^{3}+x_{0}^{2}+y_{1}^{2} y_{2} z^{3}=0 .
$$

- Now $y_{1} y_{2} \mid x_{0}^{2}$, and since $\left|\mu\left(y_{1} y_{2}\right)\right|=1$, we have $y_{1} y_{2} \mid x_{0}$. Write $x_{0}=y_{1} y_{2} w$, where $w \in \mathbb{Z}$. Substituting and dividing by $y_{1} y_{2}$, we obtain

$$
f_{2}\left(x_{1}, y_{1}, y_{2}, y_{3}, z, w\right)=x_{1} y_{2} y_{3}^{3}+w^{2} y_{1} y_{2}+y_{1} z^{3}=0 .
$$

- Since $y_{2} \mid y_{1} z^{3}$ and $\left|\mu\left(y_{1} y_{2}\right)\right|=1$, we must have $y_{2} \mid z$. Write $z=y_{2} z^{\prime}$, where $z^{\prime} \in \mathbb{Z}$, and obtain, after dividing by $y_{2}$, the relation

$$
f_{3}\left(x_{1}, y_{1}, y_{2}, y_{3}, w, z^{\prime}\right)=x_{1} y_{3}^{3}+w^{2} y_{1}+y_{1} y_{2}^{2} z^{\prime 3}=0 .
$$

- Since $y_{1}$ divides our original variables $x_{0}, x_{2}, x_{3}$, it must be coprime to $x_{1}$. Together with $\left|\mu\left(y_{1}\right)\right|=1$, the fact $y_{1} \mid x_{1} y_{3}^{3}$ implies $y_{1} \mid y_{3}$. Write $y_{3}=y_{1} y_{3}^{\prime}$, where $y_{3}^{\prime} \in \mathbb{Z}_{>0}$, and obtain

$$
f_{4}\left(x_{1}, y_{1}, y_{2}, w, z^{\prime}, y_{3}^{\prime}\right)=x_{1} y_{1}^{2} y_{3}^{\prime 3}+w^{2}+y_{2}^{2} z^{\prime 3}=0 .
$$

- Let $a=\operatorname{gcd}\left(y_{3}^{\prime}, z^{\prime}\right) \in \mathbb{Z}_{>0}$ and write $y_{3}^{\prime}=a y_{3}^{\prime \prime}$, where $y_{3}^{\prime \prime} \in \mathbb{Z}_{>0}$, and $z^{\prime}=a z^{\prime \prime}$, where $z^{\prime \prime} \in \mathbb{Z}$. This gives $f_{5}\left(x_{1}, y_{1}, y_{2}, w, z^{\prime \prime}, y_{3}^{\prime \prime}, a\right)=x_{1} y_{1}^{2} y_{3}^{\prime \prime 3} a^{3}+w^{2}+y_{2}^{2} z^{\prime \prime 3} a^{3}=0$.

- Now $a^{3} \mid w^{2}$. Writing $a=\xi_{6}^{2} \xi_{2}$, where $\xi_{2}, \xi_{6} \in \mathbb{Z}_{>0}$ with $\left|\mu\left(\xi_{2}\right)\right|=1$, gives $w=w^{\prime} \xi_{6}^{3} \xi_{2}^{2}$, where $w^{\prime} \in \mathbb{Z}$, leading to the equation

$f_{6}\left(x_{1}, y_{1}, y_{2}, z^{\prime \prime}, y_{3}^{\prime \prime}, w^{\prime}, \xi_{2}, \xi_{6}\right)=x_{1} y_{1}^{2} y_{3}^{\prime \prime 3}+w^{\prime 2} \xi_{2}+y_{2}^{2} z^{\prime \prime 3}=0$.

- Let $\xi_{5}=\operatorname{gcd}\left(y_{3}^{\prime \prime}, w^{\prime}\right) \in \mathbb{Z}_{>0}$ and write $y_{3}^{\prime \prime}=\xi_{\ell} \xi_{5}$, where $\xi_{\ell} \in \mathbb{Z}_{>0}$, and $w^{\prime}=w^{\prime \prime} \xi_{5}$, with $w^{\prime \prime} \in \mathbb{Z}$. Then

$$
f_{7}\left(x_{1}, y_{1}, y_{2}, z^{\prime \prime}, w^{\prime \prime}, \xi_{2}, \xi_{\ell}, \xi_{5}, \xi_{6}\right)=x_{1} y_{1}^{2} \xi_{\ell}^{3} \xi_{5}^{3}+w^{\prime \prime 2} \xi_{2} \xi_{5}^{2}+y_{2}^{2} z^{\prime \prime 3}=0 \text {. }
$$

- Since $\operatorname{gcd}\left(y_{3}^{\prime \prime}, z^{\prime \prime}\right)=1$, also $\operatorname{gcd}\left(\xi_{\ell} \xi_{5}, z^{\prime \prime}\right)=1$. Therefore, $\xi_{5}^{2} \mid y_{2}^{2}$, which means $\xi_{5} \mid y_{2}$, and we write $y_{2}=\xi_{1} \xi_{5}$, with $\xi_{1} \in \mathbb{Z}_{>0}$. We obtain

$$
f_{8}\left(x_{1}, y_{1}, z^{\prime \prime}, w^{\prime \prime}, \xi_{1}, \xi_{2}, \xi_{\ell}, \xi_{5}, \xi_{6}\right)=x_{1} y_{1}^{2} \xi_{\ell}^{3} \xi_{5}+w^{\prime \prime 2} \xi_{2}+\xi_{1}^{2} z^{\prime \prime 3}=0 \text {. }
$$

- Let $\xi_{3}=\operatorname{gcd}\left(w^{\prime \prime}, y_{1}\right) \in \mathbb{Z}_{>0}$. Since $\left|\mu\left(y_{1} y_{2}\right)\right|=1, \operatorname{gcd}\left(\xi_{1}, \xi_{3}\right)=1$. Hence, $\xi_{3} \mid z^{\prime \prime 3}$ and even $\xi_{3} \mid z^{\prime \prime}$. Write $w^{\prime \prime}=\tau_{2} \xi_{3}$, where $\tau_{2} \in \mathbb{Z}$, $y_{1}=\xi_{4} \xi_{3}$, where $\xi_{4} \in \mathbb{Z}_{>0}$, and $z^{\prime \prime}=\tau_{1} \xi_{3}$, where $\tau_{1} \in \mathbb{Z}$. Replacing $x_{1}=\tau_{\ell}$, where $\tau_{\ell} \in \mathbb{Z}$, we get

$$
f_{9}\left(\xi_{1}, \xi_{2}, \xi_{3}, \xi_{\ell}, \xi_{4}, \xi_{5}, \xi_{6}, \tau_{1}, \tau_{2}, \tau_{\ell}\right)=\tau_{\ell} \xi_{\ell}^{3} \xi_{4}^{2} \xi_{5}+\tau_{2}^{2} \xi_{2}+\tau_{1}^{3} \xi_{1}^{2} \xi_{3}=0 .
$$

This is the torsor equation $T\left(\xi_{i}, \tau_{i}\right)$ as in $(9.2)$.

The substitutions lead to $x_{0}, \ldots, x_{3}$ in terms of $\xi_{i}, \tau_{i}$ as in (9.3). Conversely, it is easy to check that each $\left(\xi_{i}, \tau_{i}\right)$ satisfying $T$ is mapped by $\Psi$ to a point $\mathbf{x} \in S(\mathbb{Q})$. Note that $\xi_{i} \in \mathbb{Z}_{>0}$ and $\tau_{i} \in \mathbb{Z}$. Furthermore, the coprimality conditions we introduced impose the following conditions on $\xi_{i}, \tau_{i}$ :

$$
\begin{aligned}
& \left|\mu\left(y_{1} y_{2}\right)\right|=\left|\mu\left(\xi_{1} \xi_{3} \xi_{4} \xi_{5}\right)\right|=1, \quad\left|\mu\left(\xi_{2}\right)\right|=1, \quad \operatorname{gcd}\left(\tau_{2}, \xi_{4}\right)=1, \\
& \operatorname{gcd}\left(y_{3}^{\prime \prime}, z^{\prime \prime}\right)=\operatorname{gcd}\left(\xi_{\ell} \xi_{5}, \tau_{1} \xi_{3}\right)=1, \quad \operatorname{gcd}\left(\xi_{\ell}, w^{\prime \prime}\right)=\operatorname{gcd}\left(\xi_{\ell}, \tau_{2} \xi_{3}\right)=1 .
\end{aligned}
$$

The condition $\operatorname{gcd}\left(x_{i}\right)=1$ is equivalent to $\operatorname{gcd}\left(\tau_{\ell}, \xi_{1} \xi_{2} \xi_{3} \xi_{4} \xi_{5} \xi_{6}\right)=1$. 
We obtain $\operatorname{gcd}\left(\xi_{2}, \xi_{3}\right)=1$ in the following manner: If $p \mid \xi_{2}, \xi_{3}$ for some prime $p$, then $p \mid \tau_{\ell} \xi_{\ell}^{3} \xi_{4}^{2} \xi_{5}$ by the torsor equation $T$. On the other hand, a divisor of $\xi_{3}$ cannot divide any of the factors by the coprimality conditions we found. Similarly, we conclude

$$
\operatorname{gcd}\left(\xi_{3}, \tau_{2}\right)=\operatorname{gcd}\left(\xi_{1}, \tau_{2}\right)=\operatorname{gcd}\left(\xi_{2}, \xi_{5}\right)=\operatorname{gcd}\left(\xi_{5}, \tau_{2}\right)=1 .
$$

Finally, if a prime $p$ divides two of $\xi_{2}, \xi_{4}, \tau_{1}$, we see using $T$ that $p$ must divide all of them. Since $\left|\mu\left(\xi_{2}\right)\right|=1$ and $p^{2} \mid \tau_{\ell} \xi_{\ell}^{3} \xi_{4}^{2} \xi_{5}+\tau_{1}^{3} \xi_{1}^{2} \xi_{3}$, we conclude $p \mid \tau_{2}$ which is impossible since $\operatorname{gcd}\left(\tau_{2}, \xi_{4}\right)=1$. Therefore, $\xi_{2}, \xi_{4}, \tau_{1}$ must be pairwise coprime. In the same way we derive that no two of $\xi_{1}, \xi_{2}, \xi_{\ell}$ have a common factor.

It is easy to check that we cannot derive any other coprimality condition because we could construct a solution to $T$ violating it.

Note that the conditions on $\left(\xi_{i}, \tau_{i}\right)$ are exactly the ones given in the definition of $\mathcal{T}_{2}$. Since in every step the newly introduced variables are uniquely determined, we have established a bijection between $U(\mathbb{Q})$ and $\mathcal{T}_{2}$.

The second step towards the proof of Proposition 9.3 is:

Lemma 9.5. There is a bijection between $\mathcal{T}_{1}$ and $\mathcal{T}_{2}$.

Proof. Given a point $\left(\xi_{i}^{\prime}, \tau_{j}^{\prime}\right) \in \mathcal{T}_{2}$ violating (9.7), we could replace a common prime factor $p$ of $\xi_{3}^{\prime}, \xi_{6}^{\prime}$ and $\tau_{1}^{\prime}$ by powers of $p$ as factors of $\xi_{1}^{\prime}$ and possibly $\xi_{3}^{\prime}$ such that $(9.7)$ holds. This way, we obtain a point $\left(\xi_{i}, \tau_{j}\right) \in \mathcal{T}_{1}$. This should be done in a way such that $\Psi$ maps $\left(\xi_{i}, \tau_{j}\right)$ and $\left(\xi_{i}^{\prime}, \tau_{j}^{\prime}\right)$ to the same point $\mathbf{x} \in U(\mathbb{Q})$, and such that we have an inverse map, taking care of the conditions $(9.8)$.

Let $\left(\xi_{i}, \tau_{j}\right) \in \mathcal{T}_{1}$ and $\left(\xi_{i}^{\prime}, \tau_{j}^{\prime}\right) \in \mathcal{T}_{2}$. Decompose the coordinates into their prime factors: Let

$\xi_{i}=\prod_{p} p^{n_{i p}}, \quad \tau_{j}= \pm \prod_{p} p^{m_{j p}} \quad$ and $\quad \xi_{i}^{\prime}=\prod_{p} p^{n_{i p}^{\prime}}, \quad \tau_{j}^{\prime}= \pm \prod_{p} p^{m_{j p}^{\prime}}$

where $i \in\{1,2,3, \ell, 4,5,6\}$ and $j \in\{1,2, \ell\}$. Note that (9.7) translates to $\left(n_{i p}, m_{j p}\right)$ fulfilling $n_{3 p}=n_{6 p}=0$ or $m_{1 p}=0$, and that (9.8) means that $\left(n_{i p}^{\prime}, m_{j p}^{\prime}\right)$ must fulfill $n_{1 p}^{\prime}+n_{3 p}^{\prime} \leq 1$. Furthermore, $n_{3 p}, n_{3 p}^{\prime} \in\{0,1\}$ always holds.

Define the map

$$
\begin{array}{cccc}
\Phi^{\prime}: & \mathcal{T}_{2} & \rightarrow & \mathcal{T}_{1} \\
\left(\xi_{i}^{\prime}, \tau_{i}^{\prime}\right) & \mapsto & \left(\xi_{i}, \tau_{j}\right),
\end{array}
$$

where $n_{i p}:=n_{i p}^{\prime}$ for $i \in\{2, \ell, 4,5\}$ and $m_{j p}:=m_{j p}^{\prime}$ for $j \in\{2, \ell\}$, and the values of $n_{1 p}, n_{3 p}, n_{6 p}, m_{1 p}$ depend on the size of $n_{6 p}^{\prime}$ compared to $m_{1 p}^{\prime}$, whether $m_{1 p}^{\prime}$ is even or odd, and whether $n_{3 p}^{\prime}$ is 0 or 1 :

- If $m_{1 p}^{\prime}=2 k+1, n_{6 p}^{\prime} \geq k+1, n_{3 p}^{\prime}=0$, then

$$
\left(n_{1 p}, n_{3 p}, n_{6 p}, m_{1 p}\right):=\left(n_{1 p}^{\prime}+3 k+1,1, n_{6 p}^{\prime}-k-1, m_{1 p}^{\prime}-2 k-1\right) \text {; }
$$

- if $m_{1 p}^{\prime}=2 k+1, n_{6 p}^{\prime} \geq k+1, n_{3 p}^{\prime}=1$ or $n_{6 p}^{\prime}=k, m_{1 p}^{\prime}>2 k, n_{3 p}^{\prime}=1$, then

$$
\left(n_{1 p}, n_{3 p}, n_{6 p}, m_{1 p}\right):=\left(n_{1 p}^{\prime}+3 k+2,0, n_{6 p}^{\prime}-k, m_{1 p}^{\prime}-2 k-1\right) ;
$$


- otherwise, with $n_{6 p}^{\prime}=k, m_{1 p}^{\prime}>2 k, n_{3 p}^{\prime}=0$ or $m_{1 p}^{\prime}=2 k, n_{6 p}^{\prime} \geq k$ :

$$
\left(n_{1 p}, n_{3 p}, n_{6 p}, m_{1 p}\right):=\left(n_{1 p}^{\prime}+3 k, n_{3 p}^{\prime}, n_{6 p}^{\prime}-k, m_{1 p}^{\prime}-2 k\right) .
$$

Conversely, define

$$
\Phi: \begin{array}{ccc}
\mathcal{T}_{1} & \rightarrow & \mathcal{T}_{2} \\
\left(\xi_{i}, \tau_{i}\right) & \mapsto & \left(\xi_{i}^{\prime}, \tau_{j}^{\prime}\right),
\end{array}
$$

where $n_{i p}^{\prime}:=n_{i p}$ for $i \in\{2, \ell, 4,5\}$ and $m_{j p}^{\prime}:=m_{j p}$ for $j \in\{2, \ell\}$, and the values of $n_{1 p}^{\prime}, n_{3 p}^{\prime}, n_{6 p}^{\prime}, m_{1 p}^{\prime}$ depend on $n_{1 p}$ modulo 3 and whether $n_{3 p}$ is 0 or 1:

- If $n_{1 p} \in\{3 k+1,3 k+2\}$ and $n_{3 p}=1$, then

$$
\left(n_{1 p}^{\prime}, n_{3 p}^{\prime}, n_{6 p}^{\prime}, m_{1 p}^{\prime}\right):=\left(n_{1 p}-3 k-1,0, n_{6 p}+k+1, m_{1 p}+2 k+1\right) ;
$$

- if $n_{1 p}=3 k+2$ and $n_{3 p}=0$, then

$\left(n_{1 p}^{\prime}, n_{3 p}^{\prime}, n_{6 p}^{\prime}, m_{1 p}^{\prime}\right):=\left(n_{1 p}-3 k-2,1, n_{6 p}+k, m_{1 p}+2 k+1\right)$;

- otherwise, with $n_{1 p} \in\{3 k, 3 k+1\}$ :

$$
\left(n_{1 p}^{\prime}, n_{3 p}^{\prime}, n_{6 p}^{\prime}, m_{1 p}^{\prime}\right):=\left(n_{1 p}-3 k, n_{3 p}, n_{6 p}+k, m_{1 p}+2 k\right) .
$$

It is not difficult to check that $\Phi$ and $\Phi^{\prime}$ are well-defined, that $\left(\xi_{i}, \tau_{j}\right) \in \mathcal{T}_{\widetilde{S}}(\mathbb{Z})$ and $\left(\xi_{i}^{\prime}, \tau_{j}^{\prime}\right) \in \mathcal{T}_{\widetilde{S}}(\mathbb{Z})$ correspond to the same point $\mathbf{x} \in U(\mathbb{Q})$ under the map $\Psi$, and that $\Phi$ and $\Phi^{\prime}$ are inverse to each other.

Together, Lemma 9.4 and Lemma 9.5 prove Proposition 9.3 .

\subsection{Congruences}

We use the following results from [BB04, Chapter 3] on the number of solutions of linear and quadratic congruences.

Let $\eta(a ; q)$ be the number of positive integers $n \leq q$ such that $n^{2} \equiv$ $a(\bmod q)$. Then by $\left[\right.$ BB04, Equation 3.1], we have for any $q \in \mathbb{Z}_{>0}$

$$
\eta(a ; q) \leq 2^{\omega(q)},
$$

where $\omega(q)$ is the number of distinct prime factors of $q$. Let $\vartheta$ be an arithmetic function such that

$$
\sum_{d=1}^{\infty}|(\vartheta * \mu)(d)|<\infty
$$

where $\vartheta * \mu$ is the usual Dirichlet convolution.

Lemma 9.6. Let $a, q \in \mathbb{Z}$ such that $q>0$ and $\operatorname{gcd}(a, q)=1$. For $t \geq 0$,

$$
\sum_{\substack{n \leq t \\ n \equiv a(\bmod q)}} \vartheta(n)=\frac{t}{q} \sum_{\substack{d=1 \\ \operatorname{gcd}(d, q)=1}}^{\infty}(\vartheta * \mu)(d)+O\left(\sum_{d=1}^{\infty}|(\vartheta * \mu)(d)|\right) .
$$

Proof. This is the case $\kappa=0$ of [BB04, Lemma 2].

Let $\psi(t)=\{t\}-1 / 2$ where $\{t\}$ is the fractional part of $t \in \mathbb{R}$. Let $\widetilde{\psi}(t)=\psi(t)+1$ for $t \in \mathbb{Z}$ and $\widetilde{\psi}(t)=\psi(t)$ otherwise. 
Lemma 9.7. Let $a, q \in \mathbb{Z}$, where $q>0$ and $\operatorname{gcd}(a, q)=1$. Let $b_{1}, b_{2} \in \mathbb{R}$ with $b_{1} \leq b_{2}$. Then

$$
\#\left\{n \mid b_{1} \leq n \leq b_{2}, n \equiv a(\bmod q)\right\}=\frac{b_{2}-b_{1}}{q}+r\left(b_{1}, b_{2} ; a, q\right),
$$

where

$$
r\left(b_{1}, b_{2} ; a, q\right)=\widetilde{\psi}\left(\frac{b_{1}-a}{q}\right)-\psi\left(\frac{b_{2}-a}{q}\right) .
$$

Proof. This is a slight generalization of [BB04, Lemma 3].

Lemma 9.8. Let $\epsilon>0$ and $t \geq 0$. Let $a, q \in \mathbb{Z}$ such that $q>0$ and $\operatorname{gcd}(a, q)=1$. Then

$$
\sum_{\substack{0 \leq \varrho<q \\ \operatorname{gcd}(\varrho, q)=1}} \psi\left(\frac{t-a \varrho^{2}}{q}\right) \ll_{\epsilon} q^{1 / 2+\epsilon} \quad \text { and } \sum_{\substack{0 \leq \varrho<q \\ \operatorname{gcd}(\varrho, q)=1}} \widetilde{\psi}\left(\frac{t-a \varrho^{2}}{q}\right) \ll_{\epsilon} q^{1 / 2+\epsilon}
$$

Proof. For $\psi$, this is BB04, Lemma 5].

Note that if $t \equiv a \varrho_{i}^{2}(\bmod q)$ for $i \in\{1,2\}$, then $\varrho_{1} \equiv \pm \varrho_{2}(\bmod q)$, which implies that there are at most two different values for $\varrho$ with $0 \leq \varrho<q$ such that $\left(t-a \varrho^{2}\right) / q$ is integral. Consequently, the sum for $\widetilde{\psi}$ differs from the one for $\psi$ at most by 2 .

\subsection{Summations}

Note that $\tau_{\ell}$ is determined uniquely by $T$ and the other variables, once a certain congruence is fulfilled. Therefore, our strategy is first to compute the number of possible $\tau_{2}$ depending on $\tau_{1}, \xi_{i}$ such that there exists a unique $\tau_{\ell}$ satisfying $T$. By summing over $\tau_{1}$, the number of possible $\tau_{i}$ is then computed depending on $\xi_{i}$. The summation over the variables $\xi_{i}$ is finally handled using the height zeta function.

Let

$$
X_{1}=\left(B \xi^{(-2,0,-1,3,2,1,0)}\right)^{1 / 3}, \quad X_{2}=\left(B \xi^{(0,-1,0,3,2,1,0)}\right)^{1 / 2},
$$

and

$$
X_{0}=\left(B^{-1} \xi^{(2,3,4,3,4,5,6)}\right)^{1 / 6} .
$$

Then the height conditions $\left|x_{i}\right| \leq B$ lift to

$$
\left|\left(\frac{\tau_{2}}{X_{2}}\right) X_{0}^{3}\right| \leq 1, \quad\left|\left(\frac{\tau_{2}}{X_{2}}\right)^{2}+\left(\frac{\tau_{1}}{X_{1}}\right)^{3}\right| \leq 1,
$$

and

$$
\left|X_{0}\right| \leq 1
$$

and

$$
\left|\left(\frac{\tau_{1}}{X_{1}}\right) X_{0}^{4}\right| \leq 1
$$

respectively.

Using Proposition 9.3, we can now relate the counting of rational points of bounded height on $U \subset S$ to a count on the torsor. 
Lemma 9.9. We have

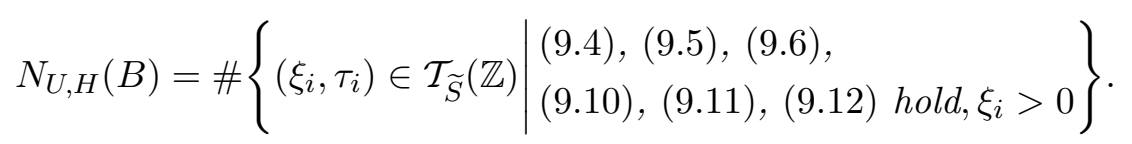

Summation over $\tau_{2}$ and $\tau_{\ell}$. Let $\xi_{i}, \tau_{1}$ satisfy the coprimality conditions (9.4), (9.5) and the height conditions (9.11) and (9.12). Let $N=N\left(\xi_{i}, \tau_{1}\right)$ be the number of pairs $\left(\tau_{2}, \tau_{\ell}\right)$ such that (9.6), 9.10) and the torsor equation $T$ are fulfilled. Then a Möbius inversion gives

$$
N=\sum_{k_{\ell} \mid \xi_{4} \xi_{5} \xi_{6}} \mu\left(k_{\ell}\right) N_{k_{\ell}}
$$

where $N_{k_{\ell}}$ has the same definition as $N$ except that $\operatorname{gcd}\left(\tau_{\ell}, \xi_{4} \xi_{5} \xi_{6}\right)=1$ is removed, and $T$ is replaced by

$$
T_{k_{\ell}}\left(\xi_{i}, \tau_{i}, k_{\ell}\right)=k_{\ell} \tau_{\ell} \xi_{\ell}^{3} \xi_{4}^{2} \xi_{5}+\tau_{2}^{2} \xi_{2}+\tau_{1}^{3} \xi_{1}^{2} \xi_{3}=0 .
$$

Note that $\tau_{2}$ together with $T_{k_{\ell}}$ defines $\tau_{\ell}$ uniquely once a certain congruence is fulfilled. Therefore,

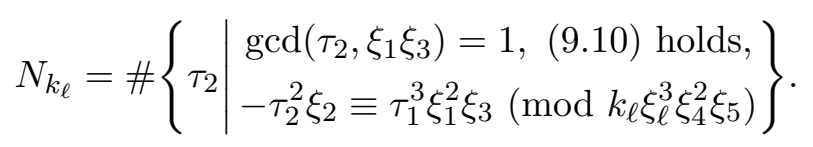

Note that

$$
\operatorname{gcd}\left(\tau_{1}^{3} \xi_{1}^{2} \xi_{3}, k_{\ell} \xi_{\ell}^{3} \xi_{4}^{2} \xi_{5}\right)=\operatorname{gcd}\left(\tau_{1}^{3} \xi_{1}^{2} \xi_{3}, \xi_{4} \xi_{5} \xi_{6}, \tau_{2}^{2} \xi_{2}\right)=\operatorname{gcd}\left(\tau_{1}, \xi_{6}, \tau_{2}^{2} \xi_{2}\right)=1
$$

and

$$
\operatorname{gcd}\left(\xi_{2}, k_{\ell} \xi_{\ell}^{3} \xi_{4}^{2} \xi_{5}\right)=\operatorname{gcd}\left(\xi_{2}, k_{\ell}\right)=\operatorname{gcd}\left(\xi_{2}, \xi_{4} \xi_{5} \xi_{6}, \tau_{1}^{3} \xi_{1}^{2} \xi_{3}\right)=1
$$

Hence, it is enough to sum over all $k_{\ell} \mid \xi_{4} \xi_{5} \xi_{6}$ with $\operatorname{gcd}\left(k_{\ell}, \tau_{1} \xi_{1} \xi_{2} \xi_{3}\right)=1$, and since $k_{\ell} \mid \xi_{4} \xi_{5} \xi_{6} \operatorname{implies} \operatorname{gcd}\left(k_{\ell}, \tau_{1}\right)=1$, we reduce to $\operatorname{gcd}\left(k_{\ell}, \xi_{1} \xi_{2} \xi_{3}\right)=1$.

This implies that there is a unique integer $\varrho$ satisfying $0<\varrho \leq k_{\ell} \xi_{\ell}^{3} \xi_{4}^{2} \xi_{5}$ and $\operatorname{gcd}\left(\varrho, k_{\ell} \xi_{\ell}^{3} \xi_{4}^{2} \xi_{5}\right)=1$ such that

$$
\tau_{2} \equiv \varrho \tau_{1} \xi_{1}\left(\bmod k_{\ell} \xi_{\ell}^{3} \xi_{4}^{2} \xi_{5}\right) \text { and }-\varrho^{2} \xi_{2} \equiv \tau_{1} \xi_{3}\left(\bmod k_{\ell} \xi_{\ell}^{3} \xi_{4}^{2} \xi_{5}\right) .
$$

We have

$$
N=\sum_{\substack{k_{\ell} \mid \xi_{4} \xi_{5} \xi_{6} \\ \operatorname{gcd}\left(k_{\ell}, \xi_{1} \xi_{2} \xi_{3}\right)=1}} \mu\left(k_{\ell}\right) \sum_{\substack{0<\varrho \leq k_{\ell} \xi_{\ell}^{3} \xi_{4}^{2} \xi_{5} \\-\varrho^{2} \xi_{2} \equiv \tau_{1} \xi_{3}\left(\bmod k_{\ell} \xi_{\ell}^{3} \xi_{4}^{2} \xi_{5}\right) \\ \operatorname{gcd}\left(\varrho, k_{\ell} \xi_{\ell}^{3} \xi_{4}^{2} \xi_{5}\right)=1}} N_{k_{\ell}}(\varrho)
$$

where

$$
N_{k_{\ell}}(\varrho)=\#\left\{\begin{array}{l}
\tau_{2} \\
\begin{array}{l}
\operatorname{gcd}\left(\tau_{2}, \xi_{1} \xi_{3}\right)=1, \\
\tau_{2} \equiv \varrho \tau_{1} \xi_{1}\left(\bmod k_{\ell} \xi_{\ell}^{3} \xi_{4}^{2} \xi_{5}\right)
\end{array}
\end{array}\right\}
$$

We also know that $\operatorname{gcd}\left(\varrho \tau_{1} \xi_{1}, k_{\ell} \xi_{\ell}^{3} \xi_{4}^{2} \xi_{5}\right)=1$. Now we can apply Lemma 9.6 to the characteristic function

$$
\chi(n)= \begin{cases}1, & \text { if } \operatorname{gcd}\left(n, \xi_{1} \xi_{3}\right)=1 \\ 0, & \text { else }\end{cases}
$$


Since

$$
\sum_{\substack{d=1 \\ \operatorname{gcd}\left(d, k_{\ell} \xi_{\ell}^{3} \xi_{4}^{2} \xi_{5}\right)=1}}^{\infty} \frac{(\chi * \mu)(d)}{d}=\prod_{\substack{p \mid \xi_{1} \xi_{3} \\ p \nmid k_{\ell} \xi_{\ell}^{3} \xi_{4}^{2} \xi_{5}}}\left(1-\frac{1}{p}\right)=\prod_{p \mid \xi_{1} \xi_{3}}\left(1-\frac{1}{p}\right)=\phi^{*}\left(\xi_{1} \xi_{3}\right),
$$

where we use $\operatorname{gcd}\left(\xi_{1} \xi_{3}, k_{\ell} \xi_{\ell}^{3} \xi_{4}^{2} \xi_{5}\right)=1$ and the notation $\phi^{*}(n):=\phi(n) / n$ as in [BB04, Equation 5.10], we conclude

$$
N_{k_{\ell}}(\varrho)=\frac{\phi^{*}\left(\xi_{1} \xi_{3}\right) X_{2}}{k_{\ell} \xi_{\ell}^{3} \xi_{4}^{2} \xi_{5}} g_{1}\left(\tau_{1} / X_{1}, X_{0}\right)+O\left(2^{\omega\left(\xi_{1} \xi_{3}\right)}\right)
$$

where $X_{2} g_{1}\left(\tau_{1} / X_{1}, X_{0}\right)$ gives the total length of the intervals in which $\tau_{2}$ must lie by 9.10$)$, with

$$
g_{1}(u, v)=\int_{\left\{t \in \mathbb{R}|| t v^{3}|\leq 1,| t^{2}+u^{3} \mid \leq 1\right\}} 1 \mathrm{~d} t .
$$

By equation $(9.9)$, the number of integers $\varrho$ with $0<\varrho \leq k_{\ell} \xi_{\ell}^{3} \xi_{4}^{2} \xi_{5}$ such that $\operatorname{gcd}\left(\varrho, k_{\ell} \xi_{\ell}^{3} \xi_{4}^{2} \xi_{5}\right)=1$ and $-\varrho^{2} \xi_{2} \equiv \tau_{1} \xi_{3}\left(\bmod k_{\ell} \xi_{\ell}^{3} \xi_{4}^{2} \xi_{5}\right)$ is at most

$$
\eta\left(\xi_{2} \xi_{3} \tau_{1} ; k_{\ell} \xi_{\ell}^{3} \xi_{4}^{2} \xi_{5}\right) \leq 2^{\omega\left(k_{\ell} \xi_{\ell}^{3} \xi_{4}^{2} \xi_{5}\right)} \leq 2^{\omega\left(\xi_{\ell} \xi_{4} \xi_{5} \xi_{6}\right)} .
$$

This gives as the first step towards the proof of Theorem 9.1

\section{LEMma 9.10.}

$$
N=\frac{X_{2}}{\xi_{\ell}^{3} \xi_{4}^{2} \xi_{5}} g_{1}\left(\tau_{1} / X_{1}, X_{0}\right) \Sigma\left(\xi_{i}, \tau_{1}\right)+O\left(2^{\omega\left(\xi_{1} \xi_{3}\right)+\omega\left(\xi_{\ell} \xi_{4} \xi_{5} \xi_{6}\right)+\omega\left(\xi_{4} \xi_{5} \xi_{6}\right)}\right),
$$

where

$$
\Sigma\left(\xi_{i}, \tau_{1}\right)=\phi^{*}\left(\xi_{1} \xi_{3}\right) \sum_{\begin{array}{c}
k_{\ell} \mid \xi_{4} \xi_{5} \xi_{6} \\
\operatorname{gcd}\left(k_{\ell}, \xi_{1} \xi_{2} \xi_{3}\right)=1
\end{array}} \frac{\mu\left(k_{\ell}\right)}{k_{\ell}} \sum_{\begin{array}{c}
0<\varrho \leq k_{\ell} \xi_{\ell}^{3} \xi_{4}^{2} \xi_{5} \\
-\varrho^{2} \xi_{2} \equiv \tau_{1} \xi_{3}\left(\bmod k_{\ell} \xi_{\ell}^{3} \xi_{4}^{2} \xi_{5}\right) \\
\operatorname{gcd}\left(\varrho, k_{\ell} \xi_{\ell}^{3} \xi_{4}^{2} \xi_{5}\right)=1
\end{array}} 1 .
$$

Now we show that the error term suffices for Theorem 9.1 We sum it over all the $\xi_{i}, \tau_{1}$ which satisfy the height conditions (9.11) and 9.12); we can ignore the coprimality conditions $(9.4),(9.5),(9.6)$. We obtain:

$$
\begin{aligned}
& \sum_{\xi_{i}} \sum_{\tau_{1}} 2^{\omega\left(\xi_{1} \xi_{3}\right)+\omega\left(\xi_{\ell} \xi_{4} \xi_{5} \xi_{6}\right)+\omega\left(\xi_{4} \xi_{5} \xi_{6}\right)} \\
\ll & \sum_{\xi_{i}} 2^{\omega\left(\xi_{1} \xi_{3}\right)+\omega\left(\xi_{\ell} \xi_{4} \xi_{5} \xi_{6}\right)+\omega\left(\xi_{4} \xi_{5} \xi_{6}\right)} \frac{X_{1}}{X_{0}^{4}} \\
= & \sum_{\xi_{i}} 2^{\omega\left(\xi_{1} \xi_{3}\right)+\omega\left(\xi_{\ell} \xi_{4} \xi_{5} \xi_{6}\right)+\omega\left(\xi_{4} \xi_{5} \xi_{6}\right)} \frac{B}{\xi^{(2,2,3,1,2,3,4)}} \\
\ll & B(\log B)^{2} \sum_{\xi_{i}, i \neq \ell} \frac{2^{\omega\left(\xi_{1} \xi_{3}\right)+\omega\left(\xi_{4} \xi_{5} \xi_{6}\right)+\omega\left(\xi_{4} \xi_{5} \xi_{6}\right)}}{\xi^{(2,2,3,0,2,3,4)}} \\
\ll & B(\log B)^{2} .
\end{aligned}
$$

For $\xi_{\ell}$, we have used the estimate

$$
\sum_{n \leq x} 2^{\omega(n)} \ll x(\log x)
$$


together with partial summation.

Therefore, we only need to consider the main term when summing over $\tau_{1}, \xi_{i}$ in order to prove Theorem 9.1 .

Summation over $\tau_{1}$. For fixed $\xi_{1}, \ldots, \xi_{6}$ satisfying (9.4) and (9.11), we sum over all $\tau_{1}$ satisfying the coprimality condition $(9.5)$ and the height condition 9.12). Let

$$
N^{\prime}=N^{\prime}\left(\xi_{i}\right)=\frac{X_{2}}{\xi_{\ell}^{3} \xi_{4}^{2} \xi_{5}} \sum_{\substack{\tau_{1}, \operatorname{gcd}\left(\tau_{1}, \xi_{2} \xi_{3} \xi_{\ell} \xi_{4} \xi_{4} \xi_{5} \xi_{6}\right)=1}} g_{1}\left(\tau_{1} / X_{1}, X_{0}\right) \Sigma\left(\xi_{i}, \tau_{1}\right) .
$$

First, we find an asymptotic formula for

$$
\mathcal{N}\left(b_{1}, b_{2}\right)=\phi^{*}\left(\xi_{1} \xi_{3}\right) \sum_{\begin{array}{c}
k_{\ell} \mid \xi_{4} \xi_{5} \xi_{6} \\
\operatorname{gcd}\left(k_{\ell}, \xi_{1} \xi_{2} \xi_{3}\right)=1
\end{array}} \frac{\mu\left(k_{\ell}\right)}{k_{\ell}} \sum_{\begin{array}{c}
0<\varrho \leq k_{\ell} \xi_{\ell}^{3} \xi_{4}^{2} \xi_{5} \\
\operatorname{gcd}\left(\varrho, k_{\ell} \xi_{\ell}^{3} \xi_{4}^{2} \xi_{5}\right)=1
\end{array}} N_{k_{\ell}}^{\prime}\left(\varrho, b_{1}, b_{2}\right),
$$

where

$$
N_{k_{\ell}}^{\prime}\left(\varrho, b_{1}, b_{2}\right)=\#\left\{\begin{array}{ll}
\tau_{1} \in\left[b_{1}, b_{2}\right] & \begin{array}{l}
\operatorname{gcd}\left(\tau_{1}, \xi_{2} \xi_{3} \xi_{\ell} \xi_{4} \xi_{5} \xi_{6}\right)=1 \\
-\varrho^{2} \xi_{2} \equiv \tau_{1} \xi_{3}\left(\bmod k_{\ell} \xi_{\ell}^{3} \xi_{4}^{2} \xi_{5}\right)
\end{array}
\end{array}\right\} .
$$

We have $\operatorname{gcd}\left(\varrho^{2} \xi_{2}, k_{\ell} \xi_{\ell}^{3} \xi_{4}^{2} \xi_{5}\right)=1$. Hence, we can replace the condition $\operatorname{gcd}\left(\tau_{1}, \xi_{2} \xi_{3} \xi_{\ell} \xi_{4} \xi_{5} \xi_{6}\right)=1$ by $\operatorname{gcd}\left(\tau_{1}, \xi_{2} \xi_{3} \xi_{6}\right)=1$ in the definition of $N_{k_{\ell}}^{\prime}\left(\varrho, b_{1}, b_{2}\right)$ and perform another Möbius inversion to obtain

$$
\begin{gathered}
\mathcal{N}\left(b_{1}, b_{2}\right)=\phi^{*}\left(\xi_{1} \xi_{3}\right) \sum_{\substack{k_{\ell} \mid \xi_{4} \xi_{5} \xi_{6} \\
\operatorname{gcd}\left(k_{\ell}, \xi_{1} \xi_{2} \xi_{3}\right)=1}} \frac{\mu\left(k_{\ell}\right)}{k_{\ell}} \sum_{\substack{k_{1} \mid \xi_{2} \xi_{3} \xi_{6} \\
\operatorname{gcd}\left(k_{1}, k_{\ell} \xi_{\ell}^{3} \xi_{4}^{2} \xi_{5}\right)=1}} \mu\left(k_{1}\right) \\
\sum_{\substack{0 \leq k_{\ell} \xi_{\ell}^{3} \xi_{4}^{2} \xi_{5} \\
\operatorname{gcd}\left(\varrho, k_{\ell} \xi_{\ell}^{3} \xi_{4}^{2} \xi_{5}\right)=1}} N_{k_{\ell}, k_{1}}^{\prime}\left(\varrho, b_{1}, b_{2}\right),
\end{gathered}
$$

where

$$
N_{k_{\ell}, k_{1}}^{\prime}\left(\varrho, b_{1}, b_{2}\right)=\#\left\{\tau_{1} \in\left[b_{1} / k_{1}, b_{2} / k_{1}\right] \mid-\varrho^{2} \xi_{2} \equiv k_{1} \tau_{1} \xi_{3}\left(\bmod k_{\ell} \xi_{\ell}^{3} \xi_{4}^{2} \xi_{5}\right)\right\} .
$$

Note that we must only sum over the $k_{1}$ with $\operatorname{gcd}\left(k_{1}, k_{\ell} \xi_{\ell}^{3} \xi_{4}^{2} \xi_{5}\right)$ because of $\operatorname{gcd}\left(\varrho^{2} \xi_{2}, k_{\ell} \xi_{\ell}^{3} \xi_{4}^{2} \xi_{5}\right)=1$.

Let $a=a\left(\xi_{i}, k_{1}, k_{\ell}\right)$ be the unique integer such that $0<a \leq k_{\ell} \xi_{\ell}^{3} \xi_{4}^{2} \xi_{5}$ and

$$
-\xi_{2} \equiv k_{1} a \xi_{3}\left(\bmod k_{\ell} \xi_{\ell}^{3} \xi_{4}^{2} \xi_{5}\right)
$$

Then $-\varrho^{2} \xi_{2} \equiv k_{1} \tau_{1} \xi_{3}\left(\bmod k_{\ell} \xi_{\ell}^{3} \xi_{4}^{2} \xi_{5}\right)$ if and only if $\tau_{1} \equiv a \varrho^{2}\left(\bmod k_{\ell} \xi_{\ell}^{3} \xi_{4}^{2} \xi_{5}\right)$. Since $\operatorname{gcd}\left(\xi_{2}, k_{\ell} \xi_{\ell}^{3} \xi_{4}^{2} \xi_{5}\right)=1$, we have $\operatorname{gcd}\left(a, k_{\ell} \xi_{\ell}^{3} \xi_{4}^{2} \xi_{5}\right)=1$. By Lemma 9.7 we conclude

$$
N_{k_{\ell}, k_{1}}^{\prime}\left(\varrho, b_{1}, b_{2}\right)=\frac{b_{2}-b_{1}}{k_{1} k_{\ell} \xi_{\ell}^{3} \xi_{4}^{2} \xi_{5}}+r\left(b_{1} / k_{1}, b_{2} / k_{1}, a \varrho^{2}, k_{\ell} \xi_{\ell}^{3} \xi_{4}^{2} \xi_{5}\right)
$$

where, by definition of $r$,

$$
r\left(b_{1} / k_{1}, b_{2} / k_{1}, a \varrho^{2}, k_{\ell} \xi_{\ell}^{3} \xi_{4}^{2} \xi_{5}\right)=\widetilde{\psi}\left(\frac{b_{1} / k_{1}-a \varrho^{2}}{k_{\ell} \xi_{\ell}^{3} \xi_{4}^{2} \xi_{5}}\right)-\psi\left(\frac{b_{2} / k_{1}-a \varrho^{2}}{k_{\ell} \xi_{\ell}^{3} \xi_{4}^{2} \xi_{5}}\right) .
$$


Let

$$
\vartheta=\vartheta\left(\xi_{i}\right)= \begin{cases}\phi^{*}\left(\xi_{1} \xi_{3}\right) \phi^{*}\left(\xi_{2} \xi_{3} \xi_{\ell} \xi_{4} \xi_{5} \xi_{6}\right) \frac{\phi^{*}\left(\xi_{4} \xi_{5} \xi_{6}\right)}{\phi^{*}\left(\operatorname{gcd}\left(\xi_{4} \xi_{5} \xi_{6}, \xi_{1} \xi_{2} \xi_{3}\right)\right)}, & 9.4 \text { holds } \\ 0, & \text { otherwise }\end{cases}
$$

Then for any $b_{1}<b_{2}$, we have

$$
\mathcal{N}\left(b_{1}, b_{2}\right)=\vartheta(\xi) \cdot\left(b_{2}-b_{1}\right)+\mathcal{R}\left(b_{1}, b_{2}\right)
$$

where

$$
\begin{aligned}
& \mathcal{R}\left(b_{1}, b_{2}\right)=\phi^{*}\left(\xi_{1} \xi_{3}\right) \sum_{\substack{k_{\ell} \mid \xi_{4} \xi_{5} \xi_{6} \\
\operatorname{gcd}\left(\tau_{\ell}, \xi_{4} \xi_{5} \xi_{6}\right)=1}} \frac{\mu\left(k_{\ell}\right)}{k_{\ell}} \sum_{\substack{k_{1} \mid \xi_{2} \xi_{3} \xi_{6} \\
\operatorname{gcd}\left(k_{1}, k_{\ell} \xi_{\ell}^{3} \xi_{4}^{2} \xi_{5}\right)=1}} \mu\left(k_{1}\right)
\end{aligned}
$$

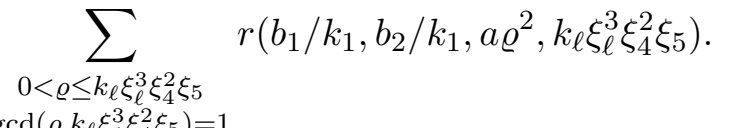

By partial summation, we obtain

$$
N^{\prime}\left(\xi_{i}\right)=\frac{\vartheta\left(\xi_{i}\right) X_{1} X_{2}}{\xi_{\ell}^{3} \xi_{4}^{2} \xi_{5}} g_{2}\left(X_{0}\right)+R^{\prime}\left(\xi_{i}\right)
$$

with

$$
g_{2}(v)=\int_{\left\{u \in \mathbb{R}|| u v^{4} \mid \leq 1\right\}} g_{1}(u, v) \mathrm{d} u
$$

and

$$
R^{\prime}\left(\xi_{i}\right)=\frac{-X_{2}}{\xi_{\ell}^{3} \xi_{4}^{2} \xi_{5}} \int_{-X_{0}^{-4}}^{X_{0}^{-4}}\left(D_{1} g_{1}\right)\left(u, X_{0}\right) \mathcal{R}\left(-X_{1} / X_{0}^{4}, X_{1} u\right) \mathrm{d} u
$$

where $D_{1} g_{1}$ is the derivation of $g_{1}$ with respect to the first variable.

LEMma 9.11. For any $\xi_{i}$ as in 9.4, 9.11), we have

$$
N^{\prime}\left(\xi_{i}\right)=\frac{\vartheta\left(\xi_{i}\right) X_{1} X_{2}}{\xi_{\ell}^{3} \xi_{4}^{2} \xi_{5}} g_{2}\left(X_{0}\right)+R^{\prime}\left(\xi_{i}\right)
$$

where the error term $R^{\prime}\left(\xi_{i}\right)$ satisfies

$$
\sum R^{\prime}\left(\xi_{i}\right)=O(B \log B) .
$$

$\xi_{i}, 9.4,9.11$ holds

Proof. By Lemma 9.8, we have

$$
\begin{aligned}
\mathcal{R}\left(b_{1}, b_{2}\right) & \ll_{\epsilon} \phi^{*}\left(\xi_{1} \xi_{3}\right) \sum_{\substack{k_{\ell} \mid \xi_{4} \xi_{5} \xi_{6} \\
\operatorname{gcd}\left(\tau_{\ell}, \xi_{4} \xi_{5} \xi_{6}\right)=1}} \frac{\left|\mu\left(k_{\ell}\right)\right|}{k_{\ell}} \sum_{\substack{k_{1} \mid \xi_{2} \xi_{3} \xi_{6} \\
\operatorname{gcd}\left(k_{1}, k_{\ell} \xi_{\ell}^{3} \xi_{4}^{2} \xi_{5}\right)=1}}\left|\mu\left(k_{1}\right)\right|\left(k_{\ell} \xi_{\ell}^{3} \xi_{4}^{2} \xi_{5}\right)^{1 / 2+\epsilon} \\
\leq & \sum_{\substack{k_{\ell} \mid \xi_{4} \xi_{5} \xi_{6} \\
\operatorname{gcd}\left(\tau_{\ell}, \xi_{4} \xi_{5} \xi_{6}\right)=1}}\left|\mu\left(k_{\ell}\right)\right| \sum_{\substack{k_{1} \mid \xi_{2} \xi_{3} \xi_{6} \\
\operatorname{gcd}\left(k_{1}, k_{\ell} \xi_{\ell}^{3} \xi_{4}^{2} \xi_{5}\right)=1}}\left|\mu\left(k_{1}\right)\right|\left(\xi_{\ell}^{3} \xi_{4}^{2} \xi_{5}\right)^{1 / 2+\epsilon} \\
\leq & 2^{\omega\left(\xi_{4} \xi_{5} \xi_{6}\right)+\omega\left(\xi_{2} \xi_{3} \xi_{6}\right)}\left(\xi_{\ell}^{3} \xi_{4}^{2} \xi_{5}\right)^{1 / 2+\epsilon} .
\end{aligned}
$$


Therefore,

$$
\begin{aligned}
R^{\prime}\left(\xi_{i}\right) & \ll \frac{X_{2}}{\xi_{\ell}^{3} \xi_{4}^{2} \xi_{5}} \int_{-X_{0}^{-4}}^{X_{0}^{-4}}\left(D_{1} g_{1}\right)\left(u, X_{0}\right) 2^{\omega\left(\xi_{4} \xi_{5} \xi_{6}\right)+\omega\left(\xi_{2} \xi_{3} \xi_{6}\right)}\left(\xi_{\ell}^{3} \xi_{4}^{2} \xi_{5}\right)^{1 / 2+\epsilon} \mathrm{d} u \\
& \ll \frac{X_{2}}{\left(\xi_{\ell}^{3} \xi_{4}^{2} \xi_{5}\right)^{1 / 2-\epsilon}} 2^{\omega\left(\xi_{4} \xi_{5} \xi_{6}\right)+\omega\left(\xi_{2} \xi_{3} \xi_{6}\right)} .
\end{aligned}
$$

Summing this over all $\xi_{i} \leq B$, we get, using (9.11),

$9.4,9.11$ hold

$$
\sum_{\xi_{i} \leq B} R^{\prime}\left(\xi_{i}\right) \ll_{\epsilon} \sum_{\xi_{i} \leq B} \frac{X_{2}}{X_{0}^{3}\left(\xi_{\ell}^{3} \xi_{4}^{2} \xi_{5}\right)^{1 / 2-\epsilon}} 2^{\omega\left(\xi_{4} \xi_{5} \xi_{6}\right)+\omega\left(\xi_{2} \xi_{3} \xi_{6}\right)}
$$

$$
\begin{aligned}
& =\sum_{\xi_{i} \leq B} \frac{B}{\xi^{(1,2,2,3 / 2-3 \epsilon, 2-2 \epsilon, 5 / 2-\epsilon, 3)}} 2^{\omega\left(\xi_{4} \xi_{5} \xi_{6}\right)+\omega\left(\xi_{2} \xi_{3} \xi_{6}\right)} \\
& \ll \sum_{\xi_{i}, i \neq 1} \frac{B \log B}{\xi^{(0,2,2,3 / 2-3 \epsilon, 2-2 \epsilon, 5 / 2-\epsilon, 3)}} 2^{\omega\left(\xi_{4} \xi_{5} \xi_{6}\right)+\omega\left(\xi_{2} \xi_{3} \xi_{6}\right)} \\
& \ll B \log B .
\end{aligned}
$$

Summation over $\xi_{i}$. Define

$$
\Delta(n)=B^{-5 / 6} \sum_{\xi_{i}, \xi^{(2,3,4,3,4,5,6)}=n} \frac{\vartheta\left(\xi_{i}\right) X_{1} X_{2}}{\xi_{\ell}^{3} \xi_{4}^{2} \xi_{5}} .
$$

We sum $N^{\prime}\left(\xi_{i}\right)$ in Lemma 9.11 over the seven variables $\xi_{i}$ such that the coprimality conditions (9.4) and the height condition (9.11) hold. Note that the definition of $\vartheta\left(\xi_{i}\right)$ ensures that the main term of $N^{\prime}\left(\xi_{i}\right)$ is zero if (9.4) is not satisfied. In view of Lemma 9.9, this implies:

LEMMA 9.12. We have

$$
N_{U, H}(B)=B^{5 / 6} \sum_{n \leq B} \Delta(n) g_{2}\left((n / B)^{1 / 6}\right)+O\left(B(\log B)^{2}\right) .
$$

\subsection{Completion of the proof}

Our argument is similar to [BB04]. We need to estimate

$$
M(t):=\sum_{n \leq t} \Delta(n)
$$

for $t>1$. Therefore, we consider the Dirichlet series $F(s):=\sum_{n=1}^{\infty} \Delta(n) n^{-s}$.

Observing

$$
\frac{X_{1} X_{2}}{\xi_{\ell}^{3} \xi_{4}^{2} \xi_{5}}=\frac{B^{5 / 6}\left(\xi^{(2,3,4,3,4,5,6)}\right)^{1 / 6}}{\xi^{(1,1,1,1,1,1,1)}}
$$

we get

$$
F(s+1 / 6)=\sum_{\xi_{i}} \frac{\vartheta\left(\xi_{i}\right)}{\xi_{1}^{2 s+1} \xi_{2}^{3 s+1} \xi_{3}^{4 s+1} \xi_{\ell}^{3 s+1} \xi_{4}^{4 s+1} \xi_{5}^{5 s+1} \xi_{6}^{6 s+1}},
$$


and writing $F(s+1 / 6)=\prod_{p} F_{p}(s+1 / 6)$ as a product of local factors, we obtain:

$$
\begin{aligned}
F_{p}(s+1 / 6)= & +\frac{(1-1 / p)^{2}}{\left(p^{\lambda_{6} s+1}-1\right)}\left(\frac{p^{\lambda_{1} s+1}}{p^{\lambda_{1} s+1}-1}+\frac{p^{\lambda_{1} s+1} p^{\lambda_{6} s+1}}{p^{\lambda_{3} s+1}\left(p^{\lambda_{1} s+1}-1\right)}\right. \\
& +\frac{p^{\lambda_{6} s+1}}{(1-1 / p) p^{\lambda_{2} s+1}}+\frac{1}{p^{\lambda_{\ell} s+1}-1}+\frac{p^{\lambda_{\ell} s+1} p^{\lambda_{6} s+1}}{p^{\lambda_{4} s+1}\left(p^{\lambda_{\ell} s+1}-1\right)} \\
& \left.+\frac{p^{\lambda_{\ell} s+1} p^{\lambda_{6} s+1}}{p^{\lambda_{5} s+1}\left(p^{\lambda_{\ell} s+1}-1\right)}\right)+\frac{1-1 / p}{p^{\lambda_{1} s+1}-1}+\frac{1-1 / p}{p^{\lambda_{\ell} s+1}-1}
\end{aligned}
$$

for any prime $p$.

Since $1 / p^{\lambda_{i} s+1}=O_{\epsilon}\left(1 / p^{1 / 2+\epsilon}\right)$ for $s \in \mathcal{H}:=\{s \in \mathbb{C} \mid \Re e(s) \geq-1 / 12+\epsilon\}$ and $i \in\{1,2,3, \ell, 4,5,6\}$, we have

$$
F_{p}(s+1 / 6)=1+\sum_{i} \frac{1}{p^{\lambda_{i} s+1}}+O_{\epsilon}\left(\frac{1}{p^{1+\epsilon}}\right)
$$

for $s \in \mathcal{H}$, and defining

$$
E(s):=\prod_{i} \zeta\left(\lambda_{i} s+1\right)=\zeta(2 s+1) \zeta(3 s+1)^{2} \zeta(4 s+1)^{2} \zeta(5 s+1) \zeta(6 s+1),
$$

we have

$$
\frac{1}{E_{p}(s)}=1-\sum_{i} \frac{1}{p^{\lambda_{i} s+1}}+O_{\epsilon}\left(\frac{1}{p^{1+\epsilon}}\right)
$$

for $s \in \mathcal{H}$. Define

$$
G(s):=F(s+1 / 6) / E(s)
$$

for $\Re e(s)>0$. Then $G$ has a holomorphic and bounded continuation to $\mathcal{H}$. Note that

$$
G(0)=\prod_{p}\left(1-\frac{1}{p}\right)^{7}\left(1+\frac{7}{p}+\frac{1}{p^{2}}\right),
$$

and that for $s \rightarrow 0$, we have

$$
E(s)=\frac{1}{\prod_{i} \lambda_{i}} s^{-7}+O\left(s^{-6}\right) .
$$

Consequently, the residue of $F(s) t^{s} / s$ at $s=1 / 6$ is

$$
\operatorname{Res}(t)=\frac{6 G(0) t^{1 / 6} Q_{1}(\log t)}{6 ! \cdot \prod_{i} \lambda_{i}}
$$

for some monic polynomial $Q_{1}$ of degree 6 .

Lemma 9.13. $M(t)=\omega_{0} \alpha(\widetilde{S}) \cdot 6 t^{1 / 6} Q_{1}(\log t)+O_{\epsilon}\left(t^{1 / 6-1 / 24+\epsilon}\right)$.

Proof. Integrating Perron's formula for $M(t)$ over $t$, we have

$$
\int_{0}^{t} M(u) \mathrm{d} u=\frac{1}{2 \pi i} \int_{1 / 6+\epsilon-i \infty}^{1 / 6+\epsilon+i \infty} F(s) \frac{t^{s+1}}{s(s+1)} \mathrm{d} s
$$

for $t>1$ and $\epsilon>0$.

We apply Cauchy's residue theorem to the rectangle with vertices

$$
1 / 12+\epsilon-i T, 1 / 12+\epsilon+i T, 1 / 6+\epsilon+i T, 1 / 6+\epsilon-i T,
$$

for some $T>1$, where $\epsilon>0$ is sufficiently small. 
By the convexity bound

$$
\zeta(1+\sigma+i \tau) \ll_{\epsilon}|\tau|^{-\sigma / 3+\epsilon}
$$

for any $\sigma \in[-1 / 2,0)$, we have

$$
F(1 / 6+\sigma+i \tau) \ll E(\sigma+i \tau) \ll_{\epsilon}|\tau|^{-9 \sigma+\epsilon}
$$

for any $\sigma \in[-1 / 12+\epsilon, 0)$, using $\left(\sum_{i} \lambda_{i}\right) / 3=9$ and that $G(\sigma+i \tau)$ is bounded.

For the ray going down from $1 / 6+\epsilon-i T$, we get

$$
\begin{aligned}
\left|\int_{1 / 6+\epsilon-i \infty}^{1 / 6+\epsilon-i T} \frac{F(s) t^{s+1}}{s(s+1)} \mathrm{d} s\right| & \leq \int_{-\infty}^{T} \frac{|F(1 / 6+\epsilon+i \sigma)|\left|t^{7 / 6+\epsilon+i \sigma}\right|}{\mid(1 / 6+\epsilon+i \sigma)(7 / 6+\epsilon+i \sigma)} \mathrm{d} \sigma \\
& \ll t^{7 / 6+\epsilon} \int_{-\infty}^{T} \frac{1}{|\sigma|^{2}} \mathrm{~d} \sigma \\
& \ll t^{7 / 6+\epsilon} T^{-1}
\end{aligned}
$$

where we use that $F(s)$ is bounded for $\Re e(s) \geq 1 / 6+\epsilon$. Integrating from $1 / 6+\epsilon+i T$ to $1 / 6+\epsilon+i \infty$ gives the same result.

For the lower edge, we estimate

$$
\begin{aligned}
\left|\int_{1 / 12+\epsilon-i T}^{1 / 6+\epsilon-i T} \frac{F(s) t^{s+1}}{s(s+1)} \mathrm{d} s\right| & \leq \int_{-1 / 12+\epsilon}^{\epsilon} \frac{|F(1 / 6+\sigma-i T)|\left|t^{7 / 6+\sigma-i T}\right|}{|(1 / 6+\sigma-i T)(7 / 6+\sigma-i T)|} \mathrm{d} \sigma \\
& \ll \frac{T^{9 / 12+\epsilon} t^{7 / 6+\epsilon}}{T^{2}},
\end{aligned}
$$

because (9.15) gives a bound for $-1 / 12+\epsilon \leq \sigma \leq-\epsilon, F(s)$ being continuous gives a bound in an $\epsilon$-neighborhood of $1 / 6-i T$, and the length of the integration interval is $1 / 12$. For the upper edge, we obtain the same bound.

For the edge on the left, we have

$$
\begin{aligned}
\left|\int_{1 / 12+\epsilon-i T}^{1 / 12+\epsilon+i T} \frac{F(s) t^{s+1}}{s(s+1)} \mathrm{d} s\right| & \leq \int_{-T}^{T} \frac{|F(1 / 12+\epsilon+i \sigma)|\left|t^{13 / 12+\epsilon+i \sigma}\right|}{\mid(1 / 12+\epsilon+i \sigma)(13 / 12+\epsilon+i \sigma)} \mathrm{d} \sigma \\
& \ll \int_{-T}^{T} \frac{|\sigma|^{9 / 12+\epsilon} t^{13 / 12+\epsilon}}{(1+|\sigma|)^{2}} \mathrm{~d} \sigma \\
& \ll t^{13 / 12+\epsilon}
\end{aligned}
$$

since the integral over $\sigma$ is bounded independently of $T$, and using (9.15) again.

Taking $T=t$, we have proved

$$
\int_{0}^{t} M(u) \mathrm{d} u=\int_{0}^{t} \operatorname{Res}(u) \mathrm{d} u+O_{\epsilon}\left(t^{13 / 12+\epsilon}\right) .
$$

But now

$$
\frac{1}{H} \int_{t-H}^{t} M(u) \mathrm{d} u \leq M(t) \leq \frac{1}{H} \int_{t}^{t+H} M(u) \mathrm{d} u,
$$

and for $H \leq t / 3$, both integrals are equal to

$$
\operatorname{Res}(t)+O_{\epsilon}\left(H t^{-5 / 6}(\log t)^{6}+H^{-1} t^{13 / 12+\epsilon}\right) .
$$


The proof of the Lemma is completed by choosing $H=t^{23 / 24}$ and noting that $\omega_{0}=G(0)$ and $\alpha(\widetilde{S})=\left(6 ! \prod_{i} \lambda_{i}\right)^{-1}$ by the definitions of $\omega_{0}$ and $\alpha(\widetilde{S})$ in Lemma 9.2

By partial summation we conclude

$$
\begin{aligned}
& \sum_{n \leq B} \Delta(n) g_{2}\left((n / B)^{1 / 6}\right) \\
& =\omega_{0} \alpha(\widetilde{S}) \cdot 6 \int_{0}^{B} g_{2}\left(u^{1 / 6} / B^{1 / 6}\right) \frac{\mathrm{d}}{\mathrm{d} u}\left(u^{1 / 6} Q_{1}(\log u)\right) \mathrm{d} u+O_{\epsilon}\left(B^{1 / 6-1 / 24+\epsilon}\right) \\
& =B^{1 / 6} \omega_{0} \alpha(\widetilde{S}) \cdot 6 \int_{0}^{1} g_{2}(v) Q_{2}(\log B+6 \log v) \mathrm{d} v+O_{\epsilon}\left(B^{1 / 6-1 / 24+\epsilon}\right)
\end{aligned}
$$

for some monic polynomial $Q_{2}$ of degree 6. Considering definitions (9.13) and (9.14), note that

$$
\omega_{\infty}=6 \int_{\{v \in \mathbb{R} \mid 0 \leq v \leq 1\}} g_{2}(v) \mathrm{d} v .
$$

Together with Lemma 9.12, this completes the proof of Theorem 9.1. 


\section{Manin's conjecture for a singular quartic surface}

\subsection{Introduction}

In this chapter, we illustrate the torsor approach to asymptotics of rational points in the case of a particular singular surface $S \subset \mathbb{P}^{4}$ of degree 4 given by:

$$
x_{0} x_{3}-x_{1} x_{4}=x_{0} x_{1}+x_{1} x_{3}+x_{2}^{2}=0 .
$$

This is a split Del Pezzo surface, with a singularity of type $\mathbf{D}_{4}$. The results first appeared in DT06,

THEOREM 10.1. The number of $\mathbb{Q}$-rational points of anticanonical height bounded by $B$ on the complement $U$ of the $\mathbb{Q}$-rational lines on $S$ (10.1) satisfies

$$
N_{U, H}(B)=c_{S, H} \cdot B \cdot Q(\log B)+O\left(B(\log B)^{3}\right) \quad \text { as } B \rightarrow \infty,
$$

where $Q$ is a monic polynomial of degree 5 , and

$$
c_{S, H}=\frac{1}{34560} \cdot \omega_{\infty} \cdot \prod_{p}(1-1 / p)^{6}\left(1+6 / p+1 / p^{2}\right)
$$

is the constant predicted by Peyre (Section 7.2), with p running through all primes and

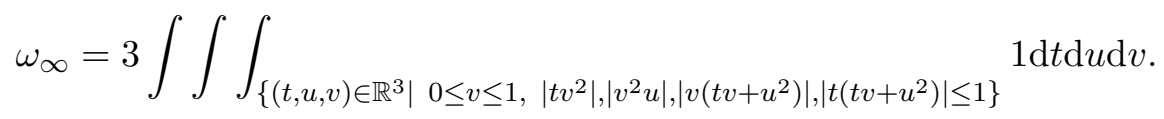

In [BB05], Manin's conjecture is proved for a non-split surface with a singularity of the same type. However, these results do not follow from each other.

In Section 10.2, we collect some facts about the geometric structure of $S$. In Section 10.3, we calculate the expected value of $c_{S, H}$ and show that Theorem 10.1 agrees with Manin's conjecture.

In our case, the universal torsor is an affine hypersurface. In Section 10.4 , we calculate its equation, stressing the relation with the geometry of $S$. We make explicit the coprimality and the height conditions. The method is more systematic than the derivation of the torsor equations in Chapter 9 , BB04, and BBD05, and should generalize to more complicated cases, e.g., other split Del Pezzo surfaces.

Note that our method gives coprimality conditions which are different from the ones in Chapter 9, BB04, and BBD05, but which are in a certain sense more natural: they are related to the set of points on $\mathcal{T}_{\widetilde{S}}$ which are stable with respect to the action of the Néron-Severi torus (in the sense of geometric invariant theory, cf. [Dol03] and [HK00]). Our conditions 
involve only coprimality of certain pairs of variables, while the other method produces a mix of square-free variables and coprimalities.

In Section 10.5, we estimate the number of integral points on the universal torsor by iterating summations over the torsor variables and using results of elementary analytic number theory. Finally we arrive at Lemma 10.9 , which is very similar in appearance to [BB04, Lemma 10] and Lemma 9.12 . In Section 10.6 we use familiar methods of height zeta functions to derive the exact asymptotic. We isolate the expected constant $c_{S, H}$ and finish the proof of Theorem 10.1 .

\subsection{Geometric background}

In this section, we collect some geometric facts concerning the surface $S$. We show that Manin's conjecture for $S$ is not a special case of available more general results for Del Pezzo surfaces.

Lemma 10.2. The surface $S$ has the following properties:

(1) It has exactly one singularity of type $\mathbf{D}_{4}$ at $q=(0: 0: 0: 0: 1)$.

(2) $S$ contains exactly two lines:

$$
\begin{aligned}
& E_{5}=\left\{x_{0}=x_{1}=x_{2}=0\right\} \text { and } E_{6}=\left\{x_{1}=x_{2}=x_{3}=0\right\}, \\
& \text { which intersect in } q .
\end{aligned}
$$

(3) The projection from the line $E_{5}$ is a birational map

$$
\begin{array}{cccc}
\phi: & S & -\rightarrow & \mathbb{P}^{2} \\
& \mathbf{x} & \mapsto & \left(x_{0}: x_{2}: x_{1}\right)
\end{array}
$$

which is defined outside $E_{5}$. It restricts to an isomorphism between

$$
U=S \backslash\left(E_{5} \cup E_{6}\right)=\left\{\mathbf{x} \in S \mid x_{1} \neq 0\right.
$$

and

$$
\mathbb{A}^{2} \cong\{(t: u: v) \mid v \neq 0\} \subset \mathbb{P}^{2},
$$

whose inverse is the restriction of

$$
\begin{aligned}
& \psi: \quad \mathbb{P}^{2} \quad \rightarrow S, \\
& (t: u: v) \mapsto\left(t v^{2}: v^{3}: v^{2} u:-v\left(t v+u^{2}\right):-t\left(t v+u^{2}\right)\right)
\end{aligned}
$$

Similar results hold for the projection from $E_{6}$.

(4) The process of resolving the singularity q gives four exceptional divisor $E_{1}, \ldots, E_{4}$ and produces the minimal desingularization $\widetilde{S}$, which is also the blow-up of $\mathbb{P}^{2}$ in five points.

Proof. Direct computations.

It will be important to know the details of the sequence of five blow-ups of $\mathbb{P}^{2}$ giving $\widetilde{S}$ as in Lemma 10.2 (4):

In order to describe the points in $\mathbb{P}^{2}$, we need the lines

$$
E_{3}=\{v=0\}, \quad A_{1}=\{u=0\}, \quad A_{2}=\{t=0\}
$$

and the curve $A_{3}=\left\{t v+u^{2}=0\right\}$.

Lemma 10.3. The following five blow-ups of $\mathbb{P}^{2}$ result in $\widetilde{S}$ :

- Blow up the intersection of $E_{3}, A_{1}, A_{3}$, giving $E_{2}$.

- Blow up the intersection of $E_{2}, E_{3}, A_{3}$, giving $E_{1}$. 
- Blow up the intersection of $E_{1}$ and $A_{3}$, giving $E_{4}$.

- Blow up the intersection of $E_{4}$ and $A_{3}$, giving $E_{6}$.

- Blow up the intersection of $E_{3}$ and $A_{2}$, giving $E_{5}$.

Here, the ordering of the first four blow-ups is fixed, and the fifth blow-up can be done at any time.

The Dynkin diagram in Figure 10.1 describes the final configuration of divisors $E_{1}, \ldots, E_{6}, A_{1}, A_{2}, A_{3}$. Here, $A_{1}, A_{2}, A_{3}$ intersect at one point.

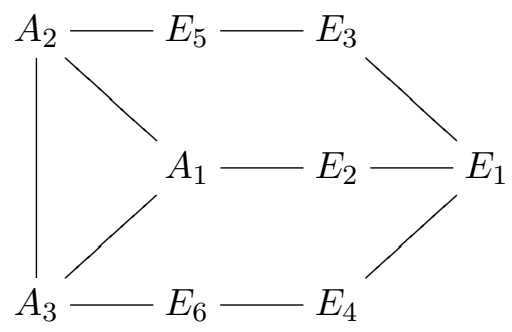

Figure 10.1. Extended Dynkin diagram

The quartic Del Pezzo surface with a singularity of type $\mathbf{D}_{4}$ is not toric (cf. Table 1.4), and Manin's conjecture does not follow from the results of [BT98]. The $\mathbf{D}_{5}$ example of [BB04 is an equivariant compactification of $\mathbb{G}_{\mathrm{a}}^{2}$ (i.e., $S$ has a Zariski open subset isomorphic to $\mathbb{A}^{2}$, and the obvious action of $\mathbb{G}_{\mathrm{a}}^{2}$ on this open subset extends to $S$ ), and thus a special case of CLT02.

Lemma 10.4. The quartic Del Pezzo surface with a singularity of type $\mathbf{D}_{4}$ is a compactification of $\mathbb{A}^{2}$, but not an equivariant compactification of $\mathbb{G}_{\mathrm{a}}^{2}$.

Proof. We follow the strategy of [HT04, Remark 3.3].

Consider the maps $\phi, \psi$ as in Lemma 10.2 3. As $\psi$ restricts to an isomorphism between $\mathbb{A}^{2}$ and the open set $U \subset S$, the surface $S$ is a compactification of $\mathbb{A}^{2}$.

If $S$ were an equivariant compactification of $\mathbb{G}_{a}^{2}$, then the projection $\phi$ from $E_{5}$ would be a $\mathbb{G}_{\mathrm{a}}^{2}$-equivariant map, giving a $\mathbb{G}_{\mathrm{a}}^{2}$-action on $\mathbb{P}^{2}$. The line $\{v=0\}$ would be invariant under this action. The only such action is the standard translation

$$
\begin{array}{cccc}
\tau: & \mathbb{P}^{2} & \rightarrow & \mathbb{P}^{2}, \\
(t: u: v) & \mapsto & (t+\alpha v: u+\beta v: v) .
\end{array}
$$

However, this action does not leave the linear series

$$
\left(t v^{2}: v^{3}: v^{2} u:-v\left(t v+u^{2}\right):-t\left(t v+u^{2}\right)\right)
$$

invariant, which can be seen after calculating

$$
\begin{aligned}
t\left(t v+u^{2}\right) \mapsto & (t+\alpha v)\left((t+\alpha v) v+(u+\beta v)^{2}\right) \\
= & t\left(t v+u^{2}\right)+2 \beta t u v+\left(\beta^{2}+\alpha\right) t v^{2}+\alpha v\left(t v+u^{2}\right) \\
& +2 \alpha \beta v^{2} u+\left(\alpha \beta^{2}+\alpha^{2}\right) v^{3}
\end{aligned}
$$

since the term tuv does not appear in the original linear series. 


\subsection{Manin's conjecture}

The following lemma shows that our result agrees with the prediction of Manin's conjecture.

Lemma 10.5. Let $S$ be the surface 10.1). Manin's conjecture for $S$ states that the number of rational points of height $\leq B$ outside the two lines is given by

$$
N_{U, H}(B) \sim c_{S, H} \cdot B(\log B)^{5},
$$

where $c_{S, H}=\alpha(\widetilde{S}) \cdot \beta(\widetilde{S}) \cdot \omega_{H}(\widetilde{S})$ with

$$
\begin{aligned}
\alpha(\widetilde{S}) & =(5 ! \cdot 4 \cdot 2 \cdot 3 \cdot 3 \cdot 2 \cdot 2)^{-1}=(34560)^{-1} \\
\beta(\widetilde{S}) & =1 \\
\omega_{H}(\widetilde{S}) & =\omega_{\infty} \cdot \prod_{p}(1-1 / p)^{6}\left(1+6 / p+1 / p^{2}\right)
\end{aligned}
$$

and

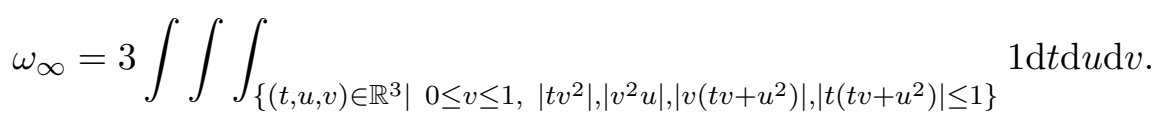

Proof. Since $S$ is split over $\mathbb{Q}$, we have $\operatorname{rk}(\operatorname{Pic}(\widetilde{S}))=6$, so the expected exponent of $\log B$ is 5 . Further, $\beta(\widetilde{S})=1$. The computation of $c_{S, H}$ is done on the desingularization $\widetilde{S}$. The constant $\alpha(\widetilde{S})$ can be calculated as $\alpha^{\prime} / \# W$ by Theorem 8.5, where $\alpha^{\prime}=1 / 180$ is the constant associated to smooth split quartic surfaces (see Theorem 8.3), and $\# W=192$ is the order of the Weyl group associated to the root system $\mathbf{D}_{4}$. The constant $\omega_{H}(\widetilde{S})$ is computed as in [BB04, Lemma 1] and Lemma 9.2 .

\subsection{The universal torsor}

By Section 7.1, the problem of counting rational points of bounded height on the surface $S$ translates into a counting problem for certain integral points on the universal torsor, subject to coprimality and height inequalities. In the first part of this section, we describe these conditions in detail. They are obtained by a process of introducing new variables which are the greatest common divisors of other variables. Geometrically, this corresponds to the realization of $\widetilde{S}$ as a blow-up of $\mathbb{P}^{2}$ in five points.

In the second part, we prove our claims.

The universal torsor $\mathcal{T}_{\widetilde{S}}$ of $S$ is an open subset of the hypersurface in $\mathbb{A}^{9}=\operatorname{Spec} \mathbb{Z}\left[\eta_{1}, \ldots, \eta_{6}, \alpha_{1}, \alpha_{2}, \alpha_{3}\right]$ defined by the equation

$$
T(\boldsymbol{\eta}, \boldsymbol{\alpha})=\alpha_{1}^{2} \eta_{2}+\alpha_{2} \eta_{3} \eta_{5}^{2}+\alpha_{3} \eta_{4} \eta_{6}^{2}=0 .
$$

The projection $\Psi: \mathcal{T}_{\widetilde{S}} \rightarrow S$ is defined by

$$
\left(\Psi^{*}\left(x_{i}\right)\right)=\left(\eta^{(2,1,2,1,2,0)} \alpha_{2}, \eta^{(4,2,3,3,2,2)}, \eta^{(3,2,2,2,1,1)} \alpha_{1}, \eta^{(2,1,1,2,0,2)} \alpha_{3}, \alpha_{2} \alpha_{3}\right),
$$

where we use the notation $\eta^{\left(n_{1}, n_{2}, n_{3}, n_{4}, n_{5}, n_{6}\right)}=\eta_{1}^{n_{1}} \eta_{2}^{n_{2}} \eta_{3}^{n_{3}} \eta_{4}^{n_{4}} \eta_{5}^{n_{5}} \eta_{6}^{n_{6}}$.

The coprimality conditions can be derived from the extended Dynkin diagram (see Figure 10.1). Two variables are allowed to have a common factor if and only if the corresponding divisors $\left(E_{i}\right.$ for $\eta_{i}$ and $A_{i}$ for $\left.\alpha_{i}\right)$ 
intersect (i.e., are connected by an edge in the diagram). Furthermore, $\operatorname{gcd}\left(\alpha_{1}, \alpha_{2}, \alpha_{3}\right)>1$ is allowed (corresponding to the fact that $A_{1}, A_{2}, A_{3}$ intersect in one point).

We will show below that there is a bijection between rational points on $U \subset S$ and integral points on an open subset of $\mathcal{T}_{\widetilde{S}}$, subject to these coprimality conditions.

We will later refer to

$$
\begin{aligned}
& \text { coprimalities between } \eta_{1}, \ldots, \eta_{6} \text { as in Figure 10.1, } \\
& \operatorname{gcd}\left(\alpha_{1}, \eta_{1} \eta_{3} \eta_{4} \eta_{5} \eta_{6}\right)=1, \\
& \operatorname{gcd}\left(\alpha_{2}, \eta_{1} \eta_{2} \eta_{3} \eta_{4} \eta_{6}\right)=1, \\
& \operatorname{gcd}\left(\alpha_{3}, \eta_{1} \eta_{2} \eta_{3} \eta_{4} \eta_{5}\right)=1 .
\end{aligned}
$$

To count the number of $\mathbf{x} \in S(\mathbb{Q})$ such that $H(\mathbf{x}) \leq B$, we must lift this condition to the universal torsor, i.e., $H(\Psi(\boldsymbol{\eta}, \boldsymbol{\alpha})) \leq B$. This is the same as

$$
\left|\eta^{(2,1,2,1,2,0)} \alpha_{2}\right| \leq B, \quad \ldots, \quad\left|\alpha_{2} \alpha_{3}\right| \leq B,
$$

using the five monomials occurring in (10.3). These have no common factors, provided the coprimality conditions are fulfilled (direct verification).

It will be useful to write the height conditions as follows. Let

$$
X_{0}=\left(\frac{\eta^{(4,2,3,3,2,2)}}{B}\right)^{1 / 3}
$$

and

$$
X_{1}=\left(B \eta^{(-1,-2,0,0,1,1)}\right)^{1 / 3}, \quad X_{2}=\left(B \eta^{(2,1,0,3,-2,4)}\right)^{1 / 3} .
$$

Then

$$
\begin{aligned}
& \left|X_{0}^{3}\right| \leq 1 \\
& \left|X_{0}^{2}\left(\alpha_{1} / X_{1}\right)\right| \leq 1 \\
& \left|X_{0}^{2}\left(\alpha_{2} / X_{2}\right)\right| \leq 1, \quad\left|X_{0}\left(X_{0}\left(\alpha_{2} / X_{2}\right)+\left(\alpha_{1} / X_{1}\right)^{2}\right)\right| \leq 1, \\
& \left|\left(\alpha_{2} / X_{2}\right)\left(X_{0}\left(\alpha_{2} / X_{2}\right)+\left(\alpha_{1} / X_{1}\right)^{2}\right)\right| \leq 1
\end{aligned}
$$

are equivalent to the five height conditions. Here we have used the torsor equation to eliminate $\alpha_{3}$ because in our counting argument we will also use that $\alpha_{3}$ is determined by the other variables.

We now prove the above claims.

Lemma 10.6. The map $\Psi$ gives a bijection between the set of points $\mathbf{x}$ of $U(\mathbb{Q})$ such that $H(\mathbf{x}) \leq B$ and the set

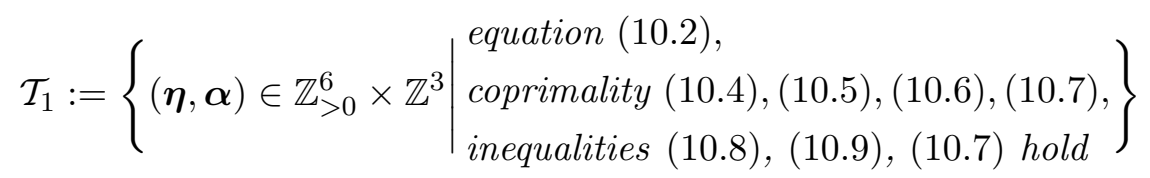

Proof. The map $\psi$ of Lemma 10.2 3 induces a bijection

$$
\psi_{0}:\left(\eta_{3}, \alpha_{1}, \alpha_{2}\right) \mapsto\left(\eta_{3}^{2} \alpha_{2}, \eta_{3}^{3}, \eta_{3}^{2} \alpha_{1}, \eta_{3} \alpha_{3}, \alpha_{2} \alpha_{3}\right),
$$

where $\alpha_{3}:=-\left(\eta_{3} \alpha_{2}+\alpha_{1}^{2}\right)$, i.e.,

$$
T_{0}:=\alpha_{1}^{2}+\eta_{3} \alpha_{2}+\alpha_{3}=0,
$$


between

$$
\left\{\left(\eta_{3}, \alpha_{1}, \alpha_{2}\right) \in \mathbb{Z}_{>0} \times \mathbb{Z}^{2} \mid \operatorname{gcd}\left(\eta_{3}, \alpha_{1}, \alpha_{2}\right)=1\right\} \text { and } U(\mathbb{Q}) \subset S(\mathbb{Q}) .
$$

The height function on $U(\mathbb{Q})$ is given by

$$
H\left(\psi_{0}\left(\eta_{3}, \alpha_{1}, \alpha_{2}\right)\right)=\frac{\max \left(\left|\eta_{3}^{2} \alpha_{2}\right|,\left|\eta_{3}^{3}\right|,\left|\eta_{3}^{2} \alpha_{1}\right|,\left|\eta_{3} \alpha_{3}\right|,\left|\alpha_{2} \alpha_{3}\right|\right)}{\operatorname{gcd}\left(\eta_{3}^{2} \alpha_{2}, \eta_{3}^{3}, \eta_{3}^{2} \alpha_{1}, \eta_{3} \alpha_{3}, \alpha_{2} \alpha_{3}\right)}
$$

The derivation of the torsor equation from the map $\psi_{0}$ together with the coprimality conditions and the lifted height function is parallel to the blowup process described in Lemma 10.3. More precisely, each line $E_{3}, A_{1}, A_{2}$ in $\mathbb{P}^{2}$ corresponds to a coordinate function $\eta_{3}, \alpha_{1}, \alpha_{2}$ vanishing in one of the lines; the blow-up of the intersection of two divisors gives an exceptional divisor $E_{i}$, corresponding to the introduction of a new variable $\eta_{i}$ as the greatest common divisor of two old variables. Two divisors are disjoint if and only if the corresponding variables are coprime. This is summarized in Table 10.1.

\begin{tabular}{|l||l|}
\hline Variables, Equations & Geometry \\
\hline \hline variables & divisors \\
initial variables & coordinate lines \\
$\eta_{3}, \alpha_{1}, \alpha_{2}$ & $E_{3}, A_{1}, A_{2}$ \\
\hline taking gcd of two variables & blowing up intersection of divisors \\
new gcd-variable & exceptional divisor \\
$\eta_{2}, \eta_{1}, \eta_{4}, \eta_{6}, \eta_{5}$ & $E_{2}, E_{1}, E_{4}, E_{6}, E_{5}$ \\
\hline extra variable & extra curve \\
$\alpha_{3}$ & $A_{3}$ \\
starting relation & starting description \\
$\alpha_{3}=-\left(\eta_{3} \alpha_{2}+\alpha_{1}^{2}\right)$ & $A_{3}=\left\{\eta_{3} \alpha_{2}+\alpha_{1}^{2}=0\right\}$ \\
final relation & torsor equation \\
$\alpha_{3} \eta_{4} \eta_{6}^{2}=-\left(\alpha_{2} \eta_{3} \eta_{5}^{2}+\alpha_{1}^{2} \eta_{2}\right)$ & $\alpha_{1}^{2} \eta_{2}+\alpha_{2} \eta_{3} \eta_{5}^{2}+\alpha_{3} \eta_{4} \eta_{6}^{2}=0$ \\
\hline
\end{tabular}

TABLE 10.1. Dictionary between gcd-process and blow-ups

This plan will now be implemented in five steps; at each step, the map

$$
\psi_{i}: \mathbb{Z}_{>0}^{i+1} \times \mathbb{Z}^{3} \rightarrow U(\mathbb{Q})
$$

gives a bijection between the following two sets:

- The set of all $\left(\eta_{j}, \alpha_{1}, \alpha_{2}, \alpha_{3}\right) \in \mathbb{Z}_{>0}^{i+1} \times \mathbb{Z}^{3}$ satisfying certain coprimality conditions (described by the extended Dynkin diagram corresponding to the $i$-th blow-up of Lemma 10.3 , an equation $T_{i}$, and

$$
H\left(\psi_{i}\left(\eta_{j}, \alpha_{j}\right)\right)=\frac{\max _{k}\left(\left|\psi_{i}\left(\eta_{j}, \alpha_{j}\right)_{k}\right|\right)}{\operatorname{gcd}\left(\psi_{i}\left(\eta_{j}, \alpha_{j}\right)_{k}\right)} \leq B .
$$

- The set of all $\mathbf{x} \in U(\mathbb{Q})$ with $H(\mathbf{x}) \leq B$.

The steps are as follows:

(1) Let $\eta_{2}:=\operatorname{gcd}\left(\eta_{3}, \alpha_{1}\right) \in \mathbb{Z}_{>0}$. Then

$$
\eta_{3}=\eta_{2} \eta_{3}^{\prime}, \quad \alpha_{1}=\eta_{2} \alpha_{1}^{\prime}, \quad \text { with } \operatorname{gcd}\left(\eta_{3}^{\prime}, \alpha_{1}^{\prime}\right)=1 \text {. }
$$


Since $\eta_{2} \mid \alpha_{3}$, we can write $\alpha_{3}=\eta_{2} \alpha_{3}^{\prime}$. Then $\alpha_{3}^{\prime}=-\left(\eta_{3}^{\prime} \alpha_{2}+\eta_{2} \alpha_{1}^{\prime 2}\right)$. After renaming the variables, we have

$$
T_{1}=\eta_{2} \alpha_{1}^{2}+\eta_{3} \alpha_{2}+\alpha_{3}=0
$$

and

$\psi_{1}:\left(\eta_{2}, \eta_{3}, \alpha_{1}, \alpha_{2}, \alpha_{3}\right) \mapsto\left(\eta_{2} \eta_{3}^{2} \alpha_{2}: \eta_{2}^{2} \eta_{3}^{3}: \eta_{2}^{2} \eta_{3}^{2} \alpha_{1}: \eta_{2} \eta_{3} \alpha_{3}: \alpha_{2} \alpha_{3}\right)$.

Here, we have eliminated the common factor $\eta_{2}$ which occurred in all five components of the image. Below, we repeat the corresponding transformation at each step.

(2) Let $\eta_{1}:=\operatorname{gcd}\left(\eta_{2}, \eta_{3}\right) \in \mathbb{Z}_{>0}$. Then

$$
\eta_{2}=\eta_{1} \eta_{2}^{\prime}, \quad \eta_{3}=\eta_{1} \eta_{3}^{\prime}, \quad \text { with } \operatorname{gcd}\left(\eta_{2}^{\prime}, \eta_{3}^{\prime}\right)=1
$$

As $\eta_{1} \mid \alpha_{3}$, we write $\alpha_{3}=\eta_{1} \alpha_{3}^{\prime}$, and we obtain:

$$
T_{2}=\eta_{2} \alpha_{1}^{2}+\eta_{3} \alpha_{2}+\alpha_{3}=0
$$

and

$\psi_{2}:\left(\eta_{1}, \eta_{2}, \eta_{3}, \alpha_{1}, \alpha_{2}, \alpha_{3}\right) \mapsto$

$$
\left(\eta_{1}^{2} \eta_{2} \eta_{3}^{2} \alpha_{2}: \eta_{1}^{4} \eta_{2}^{2} \eta_{3}^{3}: \eta_{1}^{3} \eta_{2}^{2} \eta_{3}^{2} \alpha_{1}: \eta_{1}^{2} \eta_{2} \eta_{3} \alpha_{3}: \alpha_{2} \alpha_{3}\right) .
$$

(3) Let $\eta_{4}:=\operatorname{gcd}\left(\eta_{1}, \alpha_{3}\right) \in \mathbb{Z}_{>0}$. Then

$$
\eta_{1}=\eta_{4} \eta_{1}^{\prime}, \quad \alpha_{3}=\eta_{4} \alpha_{3}^{\prime}, \quad \text { with } \operatorname{gcd}\left(\eta_{1}^{\prime}, \alpha_{3}^{\prime}\right)=1 .
$$

We get after removing ' again:

$$
T_{3}=\eta_{2} \alpha_{1}^{2}+\eta_{3} \alpha_{2}+\eta_{4} \alpha_{3}=0
$$

and

$\psi_{3}:\left(\eta_{1}, \eta_{2}, \eta_{3}, \eta_{4}, \alpha_{1}, \alpha_{2}, \alpha_{3}\right) \mapsto$

$$
\left(\eta_{1}^{2} \eta_{2} \eta_{3}^{2} \eta_{4} \alpha_{2}: \eta_{1}^{4} \eta_{2}^{2} \eta_{3}^{3} \eta_{4}^{3}: \eta_{1}^{3} \eta_{2}^{2} \eta_{3}^{2} \eta_{4}^{2} \alpha_{1}: \eta_{1}^{2} \eta_{2} \eta_{3} \eta_{4}^{2} \alpha_{3}: \alpha_{2} \alpha_{3}\right)
$$

(4) Let $\eta_{6}:=\operatorname{gcd}\left(\eta_{4}, \alpha_{3}\right) \in \mathbb{Z}_{>0}$. Then

$$
\eta_{4}=\eta_{6} \eta_{4}^{\prime}, \quad \alpha_{3}=\eta_{6} \alpha_{3}^{\prime}, \quad \text { with } \operatorname{gcd}\left(\eta_{4}^{\prime}, \alpha_{3}^{\prime}\right)=1 .
$$

We obtain

$$
T_{4}=\eta_{2} \alpha_{1}^{2}+\eta_{3} \alpha_{2}+\eta_{4} \eta_{6}^{2} \alpha_{3}=0
$$

and

$\psi_{4}:\left(\eta_{1}, \eta_{2}, \eta_{3}, \eta_{4}, \eta_{6}, \alpha_{1}, \alpha_{2}, \alpha_{3}\right) \mapsto$

$$
\left(\eta_{1}^{2} \eta_{2} \eta_{3}^{2} \eta_{4} \alpha_{2}: \eta_{1}^{4} \eta_{2}^{2} \eta_{3}^{3} \eta_{4}^{3} \eta_{6}^{2}: \eta_{1}^{3} \eta_{2}^{2} \eta_{3}^{2} \eta_{4}^{2} \eta_{6} \alpha_{1}: \eta_{1}^{2} \eta_{2} \eta_{3} \eta_{4}^{2} \eta_{6}^{2} \alpha_{3}: \alpha_{2} \alpha_{3}\right)
$$

(5) The final step is $\eta_{5}:=\operatorname{gcd}\left(\eta_{3}, \alpha_{2}\right) \in \mathbb{Z}_{>0}$, we could have done it earlier (just as the blow-up of the intersection of $E_{3}, A_{2}$ in Lemma (10.3). Then

$$
\eta_{3}=\eta_{5} \eta_{3}^{\prime}, \quad \alpha_{2}=\eta_{5} \alpha_{2}^{\prime}, \quad \text { with } \operatorname{gcd}\left(\eta_{3}^{\prime}, \alpha_{2}^{\prime}\right)=1 .
$$

We get

$$
T_{5}=\eta_{2} \alpha_{1}^{2}+\eta_{3} \eta_{5} \alpha_{2}+\eta_{4} \eta_{6}^{2} \alpha_{3}=0
$$


and

$$
\begin{aligned}
& \psi_{5}:\left(\eta_{1}, \eta_{2}, \eta_{3}, \eta_{4}, \eta_{5}, \eta_{6}, \alpha_{1}, \alpha_{2}, \alpha_{3}\right) \mapsto \\
& \left(\eta_{1}^{2} \eta_{2} \eta_{3}^{2} \eta_{4} \eta_{5}^{2} \alpha_{2}: \eta_{1}^{4} \eta_{2}^{2} \eta_{3}^{3} \eta_{4}^{3} \eta_{5}^{2} \eta_{6}^{2}: \eta_{1}^{3} \eta_{2}^{2} \eta_{3}^{2} \eta_{4}^{2} \eta_{5} \eta_{6} \alpha_{1}: \eta_{1}^{2} \eta_{2} \eta_{3} \eta_{4}^{2} \eta_{6}^{2} \alpha_{3}: \alpha_{2} \alpha_{3}\right)
\end{aligned}
$$

We observe that at each stage the coprimality conditions correspond to intersection properties of the respective divisors. The final result is summarized in Figure 10.1, which encodes data from (10.4), (10.5), (10.6), (10.7).

Note that $\psi_{5}$ is $\Psi$ from $(10.3)$. As mentioned above, $\operatorname{gcd}\left(\psi_{5}\left(\eta_{j}, \alpha_{j}\right)_{k}\right)$ (over all five components of the image) is trivial by the coprimality conditions of Figure 10.1. Therefore, $H\left(\psi_{5}(\boldsymbol{\eta}, \boldsymbol{\alpha})\right) \leq B$ is equivalent to (10.8), (10.9), (10.10.

Finally, $T_{5}$ is the torsor equation $T(10.2)$.

\subsection{Summations}

In the first step, we estimate the number of $\left(\alpha_{1}, \alpha_{2}, \alpha_{3}\right) \in \mathbb{Z}^{3}$ which fulfill the torsor equation $T(10.2)$ and the height and coprimality conditions. For fixed $\left(\alpha_{1}, \alpha_{2}\right)$, the torsor equation $T$ has a solution $\alpha_{3}$ if and only if the congruence

$$
\alpha_{1}^{2} \eta_{2}+\alpha_{2} \eta_{3} \eta_{5}^{2} \equiv 0\left(\bmod \eta_{4} \eta_{6}^{2}\right)
$$

holds and the conditions on the height and coprimalities are fulfilled.

We have already written the height conditions so that they do not depend on $\alpha_{3}$. For the coprimality, we must ensure that (10.6) and 10.7) are fulfilled.

As $\operatorname{gcd}\left(\eta_{3} \eta_{5}^{2}, \eta_{4} \eta_{6}^{2}\right)=1$, we can find the multiplicative inverse $c_{1}$ of $\eta_{3} \eta_{5}^{2}$ modulo $\eta_{4} \eta_{6}^{2}$, so that

$$
c_{1} \eta_{3} \eta_{5}^{2}=1+c_{2} \eta_{4} \eta_{6}^{2}
$$

for a suitable $c_{2}$. Choosing

$$
\begin{aligned}
& \alpha_{2}=c_{3} \eta_{4} \eta_{6}^{2}-c_{1} \alpha_{1}^{2} \eta_{2}, \\
& \alpha_{3}=c_{2} \alpha_{1}^{2} \eta_{2}-c_{3} \eta_{3} \eta_{5}^{2}
\end{aligned}
$$

gives a solution of 10.2 for any $c_{3} \in \mathbb{Z}$.

Without the coprimality conditions, the number of pairs $\left(\alpha_{2}, \alpha_{3}\right)$ satisfying $T$ and 10.10 would differ at most by $O(1)$ from $1 / \eta_{4} \eta_{6}^{2}$ of the length of the interval described by 10.10 . However, the coprimality conditions (10.6) and (10.7) impose further restrictions on the choice of $c_{3}$. A slight complication arises from the fact that because of $T$, some of the conditions are fulfilled automatically once $\boldsymbol{\eta}, \alpha_{1}$ satisfy (10.4) and (10.5).

Conditions (10.4) imply that the possibilities for a prime $p$ to divide more than one of the $\eta_{i}$ are very limited. We distinguish twelve cases, listed in Column 2 of Table 10.2

In Columns 4 and 5, we have denoted the relevant information for the divisibility of $\alpha_{2}, \alpha_{3}$ by primes $p$ which are divisors of the $\eta_{i}$ in Column 2, but of no other $\eta_{j}$ :

- "allowed" means that $\alpha_{i}$ may be divisible by $p$.

- "automatically" means that the conditions on the $\eta_{i}$ and the other $\alpha_{j}$ imply that $p \nmid \alpha_{i}$. These two cases do not impose conditions on $c_{3}$ modulo $p$. 


\begin{tabular}{|c|c||c|c|c|}
\hline case & $p \mid \ldots$ & $p \mid \alpha_{1}$ & $p \mid \alpha_{2}$ & $p \mid \alpha_{3}$ \\
\hline \hline 0 & - & allowed & allowed & allowed \\
$i$ & $\eta_{1}$ & restriction & restriction & restriction \\
$i i$ & $\eta_{2}$ & allowed & restriction & automatically \\
$i i i$ & $\eta_{3}$ & restriction & restriction & automatically \\
$i v$ & $\eta_{4}$ & restriction & automatically & restriction \\
$v$ & $\eta_{5}$ & restriction & allowed & automatically \\
$v i$ & $\eta_{6}$ & restriction & automatically & allowed \\
\hline$v i i$ & $\eta_{1}, \eta_{2}$ & restriction & restriction & automatically \\
$v i i i$ & $\eta_{1}, \eta_{3}$ & restriction & restriction & automatically \\
$i x$ & $\eta_{1}, \eta_{4}$ & restriction & automatically & restriction \\
$x$ & $\eta_{3}, \eta_{5}$ & restriction & restriction & automatically \\
$x i$ & $\eta_{4}, \eta_{6}$ & restriction & automatically & restriction \\
\hline
\end{tabular}

TABLE 10.2. Coprimality conditions

- "restriction" means that $c_{3}$ is not allowed to be in a certain congruence class modulo $p$ in order to fulfill the condition that $p$ must not divide $\alpha_{i}$.

The information in the table is derived as follows:

- If $p \mid \eta_{3}$, then $p \nmid c_{2}$ from 10.11, and $p \nmid \alpha_{1} \eta_{2}$ because of (10.4), (10.5), so by 10.13), $p \nmid \alpha_{3}$ independently of the choice of $c_{3}$. Since $p \nmid \eta_{4} \eta_{6}^{2}$, we see from 10.12 that $p \mid \alpha_{2}$ for one in $p$ subsequent choices of $c_{3}$ which we must therefore exclude. This explains cases iii and viii.

- In case vii, the same is true for $\alpha_{2}$. More precisely, we see that we must exclude $c_{3} \equiv 0(\bmod p)$. By 10.13$), p \nmid c_{3}$ implies that $p \nmid \alpha_{3}$, so we do not need another condition on $c_{3}$.

- In case $i$, we see that $p \mid \alpha_{2}$ for one in $p$ subsequent choices of $c_{3}$, and the same holds for $\alpha_{3}$. However, in this case, $p$ cannot divide $\alpha_{2}, \alpha_{3}$ for the same choice of $c_{3}$, as we can see by considering $T$ : since $p \nmid \alpha_{1}^{2} \eta_{2}$, it is impossible that $p \mid \alpha_{2}, \alpha_{3}$. Consequently, we must exclude two out of $p$ subsequent choices of $p$ in order to fulfill $p \nmid \alpha_{2}, \alpha_{3}$.

- In the other cases, the arguments are similar.

The number of $\left(\alpha_{2}, \alpha_{3}\right) \in \mathbb{Z}^{2}$ subject to $\left.T,(10.6), 10.7\right),(10.10)$ equals the number of $c_{3}$ such that $\alpha_{2}, \alpha_{3}$ as in (10.12), (10.13) satisfy these conditions. This can be estimated as $1 / \eta_{4} \eta_{6}^{2}$ of the length of the interval described by 10.10 , multiplied by a product of local factors whose values can be read off from Columns 2, 4, 5 of Table 10.2 , the divisibility properties of $\eta_{i}$ by $p$ determine whether zero, one or two out of $p$ subsequent values of $c_{3}$ have to be excluded. Different primes can be considered separately, and we define

$$
\vartheta_{1, p}:= \begin{cases}1-2 / p, & \text { case } i \\ 1-1 / p, & \text { cases } i i, \ldots, i v, v i, \ldots, x i \\ 1, & \text { cases } 0, v\end{cases}
$$


Let

$$
\vartheta_{1}(\boldsymbol{\eta})=\prod_{p} \vartheta_{1, p}
$$

be the product of these local factors, and

$$
g_{1}(u, v)=\int_{\left\{t \in \mathbb{R}|| t v^{2}|,| t\left(t v+u^{2}\right)|,| v\left(t v+u^{2}\right) \mid \leq 1\right\}} 1 \mathrm{~d} t .
$$

Let $\omega(n)$ denote the number of primes dividing $n$.

Lemma 10.7. For fixed $\left(\boldsymbol{\eta}, \alpha_{1}\right) \in \mathbb{Z}_{>0}^{6} \times \mathbb{Z}$ as in 10.4), 10.5), 10.8), (10.9), the number of $\left(\alpha_{2}, \alpha_{3}\right) \in \mathbb{Z}^{2}$ satisfying $T$, (10.6), (10.7), (10.10) is

$$
\mathcal{N}_{1}\left(\boldsymbol{\eta}, \alpha_{1}\right)=\frac{\vartheta_{1}(\boldsymbol{\eta}) X_{2}}{\eta_{4} \eta_{6}^{2}} g_{1}\left(\alpha_{1} / X_{1}, X_{0}\right)+O\left(2^{\omega\left(\eta_{1} \eta_{2} \eta_{3} \eta_{4} \eta_{6}\right)}\right) .
$$

The sum of error terms for all possible values of $\left(\boldsymbol{\eta}, \alpha_{1}\right)$ is $\ll B(\log B)^{3}$.

Proof. The number of $c_{3}$ such that the resulting $\alpha_{2}, \alpha_{3}$ satisfy 10.10 differs from $\frac{X_{2}}{\eta_{4} \eta_{6}^{2}} g_{1}\left(\alpha_{1} / X_{1}, X_{0}\right)$ by at most $O(1)$.

Each $\vartheta_{1, p} \neq 1$ corresponds to a congruence condition on $c_{3}$ imposed by one of the cases $i, \ldots, i v, v i, \ldots, x i$. For each congruence condition, the actual ratio of allowed $c_{3}$ can differ at most by $O(1)$ from the $\vartheta_{1, p}$. The total number of these primes $p$ is

$$
\omega\left(\eta_{1} \eta_{2} \eta_{3} \eta_{4} \eta_{6}\right) \ll 2^{\omega\left(\eta_{1} \eta_{2} \eta_{3} \eta_{4} \eta_{6}\right)}
$$

which is independent of $\eta_{5}$ since any prime dividing only $\eta_{5}$ contributes a trivial factor (see case $v$ ).

Using the estimate 10.9 for $\alpha_{1}$ in the first step and ignoring 10.4 10.5, which can only increase the error term, we obtain:

$$
\sum_{\boldsymbol{\eta}} \sum_{\alpha_{1}} 2^{\omega\left(\eta_{1} \eta_{2} \eta_{3} \eta_{4} \eta_{6}\right)} \leq \sum_{\boldsymbol{\eta}} \frac{B \cdot 2^{\omega\left(\eta_{1} \eta_{2} \eta_{3} \eta_{4} \eta_{6}\right)}}{\eta^{(3,2,2,2,1,1)}} \ll B(\log B)^{3} .
$$

Here, we use $2^{\omega(n)} \ll_{\epsilon} n^{\epsilon}$ for the summations over $\eta_{1}, \eta_{2}, \eta_{3}, \eta_{4}$. For $\eta_{6}$, we employ

$$
\sum_{n \leq x} 2^{\omega(n)} \ll x(\log x)
$$

together with partial summation, contributing a factor $(\log B)^{2}$, while the summation over $\eta_{5}$ gives another factor $\log B$.

Next, we sum over all $\alpha_{1}$ subject to the coprimality condition 10.5 and the height condition 10.9p. Let

$$
g_{2}(v)=\int_{\left\{u \in \mathbb{R}|| v^{2} u \mid \leq 1\right\}} g_{1}(u, v) \mathrm{d} u .
$$

Similar to our discussion for $\alpha_{2}, \alpha_{3}$, the number of possible values for $\alpha_{1}$ as in (10.9), while ignoring (10.5) for the moment, is $X_{1} g_{2}\left(X_{0}\right)+O(1)$.

None of the coprimality conditions are fulfilled automatically, and only common factors with $\eta_{2}$ are allowed (see Column 3 of Table 10.2). Therefore, each prime factor of $\eta_{1} \eta_{3} \eta_{4} \eta_{5} \eta_{6}$ reduces the number of allowed $\alpha_{1}$ by a factor 
of $\vartheta_{2, p}=1-1 / p$ with an error of at most $O(1)$. For all other primes $p$, let $\vartheta_{2, p}=1$, and let

$$
\vartheta_{2}(\boldsymbol{\eta})=\prod_{p} \vartheta_{2, p} \quad \text { and } \quad \vartheta(\boldsymbol{\eta})= \begin{cases}\vartheta_{1}(\boldsymbol{\eta}) \cdot \vartheta_{2}(\boldsymbol{\eta}), & 10.4 \text { holds } \\ 0, & \text { otherwise. }\end{cases}
$$

LEMma 10.8. For fixed $\eta \in \mathbb{Z}_{>0}^{6}$ as in (10.4), (10.8), the sum of $\mathcal{N}_{1}\left(\boldsymbol{\eta}, \alpha_{1}\right)$ over all $\alpha_{1} \in \mathbb{Z}$ satisfying (10.5), (10.9) is

$$
\mathcal{N}_{2}(\boldsymbol{\eta}):=\frac{\vartheta(\boldsymbol{\eta}) X_{1} X_{2}}{\eta_{4} \eta_{6}^{2}} g_{2}\left(X_{0}\right)+\mathcal{R}_{2}(\boldsymbol{\eta})
$$

where the sum of error terms $\mathcal{R}_{2}(\boldsymbol{\eta})$ over all possible $\boldsymbol{\eta}$ is $\ll B \log B$.

Proof. Let

$$
\mathcal{N}\left(b_{1}, b_{2}\right)=\vartheta_{1}(\boldsymbol{\eta}) \cdot \#\left\{\alpha_{1} \in\left[b_{1}, b_{2}\right] \mid \operatorname{gcd}\left(\alpha_{1}, \eta_{1} \eta_{3} \eta_{4} \eta_{5} \eta_{6}\right)=1\right\} .
$$

Using Möbius inversion, this is estimated as

$$
\mathcal{N}\left(b_{1}, b_{2}\right)=\vartheta_{1}(\boldsymbol{\eta}) \cdot \vartheta_{2}(\boldsymbol{\eta}) \cdot\left(b_{2}-b_{1}\right)+\mathcal{R}\left(b_{1}, b_{2}\right)
$$

with $\mathcal{R}\left(b_{1}, b_{2}\right)=O\left(2^{\omega\left(\eta_{1} \eta_{3} \eta_{4} \eta_{5} \eta_{6}\right)}\right)$. By partial summation,

$$
\mathcal{N}_{2}(\boldsymbol{\eta})=\frac{\vartheta(\boldsymbol{\eta}) X_{1} X_{2}}{\eta_{4} \eta_{6}^{2}} g_{2}\left(X_{0}\right)+\mathcal{R}_{2}(\boldsymbol{\eta})
$$

with

$$
\mathcal{R}_{2}(\boldsymbol{\eta})=\frac{-X_{2}}{\eta_{4} \eta_{6}^{2}} \int_{\left\{u|| X_{0}^{2} u \mid \leq 1\right\}}\left(D_{1} g_{1}\right)\left(u, X_{0}\right) \mathcal{R}\left(-X_{1} / X_{0}^{2}, X_{1} u\right) \mathrm{d} u
$$

where $D_{1} g_{1}$ is the partial derivative of $g_{1}$ with respect to the first variable. Using the above bound for $\mathcal{R}\left(b_{1}, b_{2}\right)$, we obtain:

$$
\mathcal{R}_{2}(\boldsymbol{\eta}) \ll \frac{X_{2}}{\eta_{4} \eta_{6}^{2}} 2^{\omega\left(\eta_{1} \eta_{3} \eta_{4} \eta_{5} \eta_{6}\right)} .
$$

Summing this over all $\boldsymbol{\eta}$ as in 10.8 while ignoring (10.4 which can only enlarge the sum, we obtain

$$
\sum_{\boldsymbol{\eta}} \mathcal{R}_{2}(\boldsymbol{\eta}) \ll \sum_{\boldsymbol{\eta}} \frac{X_{2} \cdot 2^{\omega\left(\eta_{1} \eta_{3} \eta_{4} \eta_{5} \eta_{6}\right)}}{\eta_{4} \eta_{6}^{2} X_{0}^{2}}=\sum_{\boldsymbol{\eta}} \frac{B \cdot 2^{\omega\left(\eta_{1} \eta_{3} \eta_{4} \eta_{5} \eta_{6}\right)}}{\eta^{(2,1,2,2,2,2)}} \ll B \log B .
$$

In the first step, we use $X_{0} \leq 1$.

Let

$$
\Delta(n)=B^{-2 / 3} \sum_{\eta_{i}, \eta^{(4,2,3,3,2,2)}=n} \frac{\vartheta(\boldsymbol{\eta}) X_{1} X_{2}}{\eta_{4} \eta_{6}^{2}}=\sum_{\eta_{i}, \eta^{(4,2,3,3,2,2)}=n} \frac{\vartheta(\boldsymbol{\eta})\left(\eta^{(4,2,3,3,2,2)}\right)^{1 / 3}}{\eta^{(1,1,1,1,1,1)}} .
$$

In view of Lemma 10.6, the number of rational points of bounded height on $U$ can be estimated by summing the result of Lemma 10.8 over all suitable $\boldsymbol{\eta}$. The error term is the combination of the error terms in Lemmas 10.7 and 10.8 .

Lemma 10.9. We have

$$
N_{U, H}(B)=B^{2 / 3} \sum_{n \leq B} \Delta(n) g_{2}\left((n / B)^{1 / 3}\right)+O\left(B(\log B)^{3}\right) .
$$




\subsection{Completion of the proof}

We need an estimate for

$$
M(t):=\sum_{n \leq t} \Delta(n)
$$

Consider the Dirichlet series $F(s):=\sum_{n=1}^{\infty} \Delta(n) n^{-s}$. Using

$$
F(s+1 / 3)=\sum_{\boldsymbol{\eta}} \frac{\vartheta(\boldsymbol{\eta})}{\eta_{1}^{4 s+1} \eta_{2}^{2 s+1} \eta_{3}^{3 s+1} \eta_{4}^{3 s+1} \eta_{5}^{2 s+1} \eta_{6}^{2 s+1}},
$$

we write $F(s+1 / 3)=\prod_{p} F_{p}(s+1 / 3)$ as its Euler product. To obtain $F_{p}(s+1 / 3)$ for a prime $p$, we need to restrict this sum to the terms in which all $\eta_{i}$ are powers of $p$. Note that $\vartheta(\boldsymbol{\eta})$ is non-zero if and only if the divisibility of $\eta_{i}$ by $p$ falls into one of the twelve cases described in Table 10.2 . The value of $\vartheta(\boldsymbol{\eta})$ only depends on these cases.

Writing $F_{p}(s+1 / 3)=\sum_{i=0}^{11} F_{p, i}(s+1 / 3)$, we have for example:

$$
\begin{aligned}
& F_{p, 0}(s+1 / 3)=1 \\
& F_{p, 1}(s+1 / 3)=\sum_{j=1}^{\infty} \frac{(1-1 / p)(1-2 / p)}{p^{j(4 s+1)}}=\frac{(1-1 / p)(1-2 / p)}{p^{4 s+1}-1}, \\
& F_{p, 7}(s+1 / 3)=\sum_{j, k=1}^{\infty} \frac{(1-1 / p)^{2}}{p^{j(4 s+1)} p^{k(2 s+1)}}=\frac{(1-1 / p)^{2}}{\left(p^{4 s+1}-1\right)\left(p^{2 s+1}-1\right)} .
\end{aligned}
$$

The other cases are similar, giving

$$
\begin{aligned}
F_{p}(s+1 / 3)= & +\frac{1-1 / p}{p^{4 s+1}-1}\left((1-2 / p)+\frac{1-1 / p}{p^{2 s+1}-1}+2 \frac{1-1 / p}{p^{3 s+1}-1}\right) \\
& +\frac{1-1 / p}{p^{2 s+1}-1}+2 \frac{(1-1 / p)^{2}}{p^{3 s+1}-1}+2 \frac{1-1 / p}{p^{2 s+1}-1}+2 \frac{(1-1 / p)^{2}}{\left(p^{2 s+1}-1\right)^{2}} .
\end{aligned}
$$

Defining

$E(s):=\zeta(4 s+1) \zeta(3 s+1)^{2} \zeta(2 s+1)^{3} \quad$ and $\quad G(s):=F(s+1 / 3) / E(s)$, we see as in Section 9.6 that the residue of $F(s) t^{s} / s$ at $s=1 / 3$ is

$$
\operatorname{Res}(t)=\frac{3 G(0) t^{1 / 3} Q_{1}(\log t)}{5 ! \cdot 4 \cdot 2 \cdot 3 \cdot 3 \cdot 2 \cdot 2}
$$

for a monic $Q_{1} \in \mathbb{R}[x]$ of degree 5 . By Lemma $10.5, \alpha(S)=\frac{1}{5 ! \cdot 4 \cdot 2 \cdot 3 \cdot 3 \cdot 2 \cdot 2}$. By a Tauberian argument as in Lemma 9.13 .

Lemma 10.10. $M(t)=\operatorname{Res}(t)+O\left(t^{1 / 3-\delta}\right)$ for some $\delta>0$.

By partial summation,

$$
\sum_{n \leq B} \Delta(n) g_{2}\left((n / B)^{1 / 3}\right)=\alpha(S) G(0) B^{1 / 3} Q(\log B) \cdot 3 \int_{0}^{1} g_{2}(v) \mathrm{d} v+O\left(B^{\frac{1}{3}-\delta}\right)
$$

for a monic polynomial $Q$ of degree 5 . We identify $\omega_{H}(S)$ from

$$
G(0)=\prod_{p}\left(1-\frac{1}{p}\right)^{6}\left(1+\frac{6}{p}+\frac{1}{p^{2}}\right), \text { and } \omega_{\infty}=3 \int_{0}^{1} g_{2}(v) \mathrm{d} v .
$$

Together with Lemma 10.9, this completes the proof of Theorem 10.1 . 


\section{Bibliography}

[AN04] V. Alexeev and V. V. Nikulin. Classification of log del Pezzo surfaces of index $\leq 2$, arXiv:math.AG/0406536, 2004.

[Art66] M. Artin. On isolated rational singularities of surfaces. Amer. J. Math., 88:129136, 1966.

[BB04] R. de la Bretèche and T. D. Browning. On Manin's conjecture for singular del Pezzo surfaces of degree four, I. Mich. Math. J., to appear, arXiv:math.NT/0412086, 2004.

[BB05] R. de la Bretèche and T. D. Browning. On Manin's conjecture for singular del Pezzo surfaces of degree four, II. Math. Proc. Camb. Phil. Soc., to appear, arXiv:math.NT/0502510, 2005.

[BBD05] R. de la Bretèche, T. D. Browning, and U. Derenthal. On Manin's conjecture for a certain singular cubic surface. Ann. Sci. École Norm. Sup. (4), arXiv:math.NT/0509370, 2005.

[BF04] R. de la Bretèche and É. Fouvry. L'éclaté du plan projectif en quatre points dont deux conjugués. J. Reine Angew. Math., 576:63-122, 2004.

[Bir62] B. J. Birch. Forms in many variables. Proc. Roy. Soc. Ser. A, 265:245-263, 1961/1962.

[BM90] V. V. Batyrev and Yu. I. Manin. Sur le nombre des points rationnels de hauteur borné des variétés algébriques. Math. Ann., 286(1-3):27-43, 1990.

[BP04] V. V. Batyrev and O. N. Popov. The Cox ring of a del Pezzo surface. In Arithmetic of higher-dimensional algebraic varieties (Palo Alto, CA, 2002), volume 226 of Progr. Math., pages 85-103. Birkhäuser Boston, Boston, MA, 2004.

[Bre98] R. de la Bretèche. Sur le nombre de points de hauteur bornée d'une certaine surface cubique singulière. Astérisque, (251):51-77, 1998. Nombre et répartition de points de hauteur bornée (Paris, 1996).

[Bre02] R. de la Bretèche. Nombre de points de hauteur bornée sur les surfaces de del Pezzo de degré 5. Duke Math. J., 113(3):421-464, 2002.

[Bro89] K. S. Brown. Buildings. Springer-Verlag, New York, 1989.

[Bro04] T. D. Browning. The density of rational points on a certain singular cubic surface, arXiv:math.NT/0404245, 2004.

[Bro05] T. D. Browning. An overview of Manin's conjecture for del Pezzo surfaces, arXiv:math.NT/0511041, 2005.

[BT95] V. V. Batyrev and Yu. Tschinkel. Rational points of bounded height on compactifications of anisotropic tori. Internat. Math. Res. Notices, (12):591-635, 1995.

[BT98] V. V. Batyrev and Yu. Tschinkel. Manin's conjecture for toric varieties. J. Algebraic Geom., 7(1):15-53, 1998.

[BW79] J. W. Bruce and C. T. C. Wall. On the classification of cubic surfaces. J. London Math. Soc. (2), 19(2):245-256, 1979.

[Cay69] A. Cayley. A memoir on cubic surfaces. Philos. Trans. Roy. Soc. London, 159:231-326, 1869.

[CLT02] A. Chambert-Loir and Yu. Tschinkel. On the distribution of points of bounded height on equivariant compactifications of vector groups. Invent. Math., 148(2):421-452, 2002.

[Cox95] D. A. Cox. The homogeneous coordinate ring of a toric variety. J. Algebraic Geom., 4(1):17-50, 1995. 
[CT88] D. F. Coray and M. A. Tsfasman. Arithmetic on singular Del Pezzo surfaces. Proc. London Math. Soc. (3), 57(1):25-87, 1988.

[CTS80] J.-L. Colliot-Thélène and J.-J. Sansuc. La descente sur les variétés rationnelles. In Journées de Géometrie Algébrique d'Angers, Juillet 1979/Algebraic Geometry, Angers, 1979, pages 223-237. Sijthoff \& Noordhoff, Alphen aan den Rijn, 1980.

[CTS87] J.-L. Colliot-Thélène and J.-J. Sansuc. La descente sur les variétés rationnelles. II. Duke Math. J., 54(2):375-492, 1987.

[Der05] U. Derenthal. Manin's conjecture for a certain singular cubic surface, arXiv:math.NT/0504016, 2005.

[Der06a] U. Derenthal. On a constant arising in Manin's conjecture for Del Pezzo surfaces, 2006.

[Der06b] U. Derenthal. On the Cox ring of Del Pezzo surfaces, arXiv:math.AG/0603111, 2006.

[Der06c] U. Derenthal. Singular Del Pezzo surfaces whose universal torsors are hypersurfaces, arXiv:math.AG/0604194, 2006.

[Der06d] U. Derenthal. Universal torsors of Del Pezzo surfaces and homogeneous spaces, arXiv:math.AG/0604195, 2006.

[Dol03] I. Dolgachev. Lectures on invariant theory, volume 296 of London Mathematical Society Lecture Note Series. Cambridge University Press, Cambridge, 2003.

[DP80] M. Demazure and H. C. Pinkham, editors. Séminaire sur les Singularités des Surfaces, volume 777 of Lecture Notes in Mathematics. Springer, Berlin, 1980. Held at the Centre de Mathématiques de l'École Polytechnique, Palaiseau, 19761977.

[DT06] U. Derenthal and Yu. Tschinkel. Universal torsors over Del Pezzo surfaces and rational points. In Equidistribution in Number Theory, An Introduction (Montréal, 2005), to appear, NATO Sci. Ser. II Math. Phys. Chem., Dordrecht, 2006. Springer.

[DV34] P. Du Val. On isolated singularities of surfaces which do not affect the conditions of adjunction (parts ii and iii). Proc. Cambridge Philos. Soc., 30:460-465, 483491, 1934.

[EKW04] E. J. Elizondo, K. Kurano, and K. Watanabe. The total coordinate ring of a normal projective variety. J. Algebra, 276(2):625-637, 2004.

[FM02] R. Friedman and J. W. Morgan. Exceptional groups and del Pezzo surfaces. In Symposium in Honor of C. H. Clemens (Salt Lake City, UT, 2000), volume 312 of Contemp. Math., pages 101-116. Amer. Math. Soc., Providence, RI, 2002.

[FMT89] J. Franke, Yu. I. Manin, and Yu. Tschinkel. Rational points of bounded height on Fano varieties. Invent. Math., 95(2):421-435, 1989.

[Fou98] É. Fouvry. Sur la hauteur des points d'une certaine surface cubique singulière. Astérisque, (251):31-49, 1998. Nombre et répartition de points de hauteur bornée (Paris, 1996).

[Fre53] H. Freudenthal. Sur le groupe exceptionnel $E_{7}$. Nederl. Akad. Wetensch. Proc. Ser. A. 56=Indagationes Math., 15:81-89, 1953.

[Fu193] W. Fulton. Introduction to toric varieties, volume 131 of Annals of Mathematics Studies. Princeton University Press, Princeton, NJ, 1993. The William H. Roever Lectures in Geometry.

[GJ00] E. Gawrilow and M. Joswig. polymake: a framework for analyzing convex polytopes. In Polytopes - combinatorics and computation (Oberwolfach, 1997), volume 29 of DMV Sem., pages 43-73. Birkhäuser, Basel, 2000.

[Har77] R. Hartshorne. Algebraic geometry. Springer-Verlag, New York, 1977. Graduate Texts in Mathematics, No. 52.

[Has04] B. Hassett. Equations of universal torsors and Cox rings. In Mathematisches Institut, Georg-August-Universität Göttingen: Seminars Summer Term 2004, pages 135-143. Universitätsdrucke Göttingen, Göttingen, 2004.

[HB03] D. R. Heath-Brown. The density of rational points on Cayley's cubic surface. In Proceedings of the Session in Analytic Number Theory and Diophantine Equations, volume 360 of Bonner Math. Schriften, page 33, Bonn, 2003. Univ. Bonn. 
[HBM99] D. R. Heath-Brown and B. Z. Moroz. The density of rational points on the cubic surface $X_{0}^{3}=X_{1} X_{2} X_{3}$. Math. Proc. Cambridge Philos. Soc., 125(3):385-395, 1999.

[HK00] Y. Hu and S. Keel. Mori dream spaces and GIT. Michigan Math. J., 48:331-348, 2000. Dedicated to William Fulton on the occasion of his 60th birthday.

[HT04] B. Hassett and Yu. Tschinkel. Universal torsors and Cox rings. In Arithmetic of higher-dimensional algebraic varieties (Palo Alto, CA, 2002), volume 226 of Progr. Math., pages 149-173. Birkhäuser Boston, Boston, MA, 2004.

[Kle66] S. L. Kleiman. Toward a numerical theory of ampleness. Ann. of Math. (2), 84:293-344, 1966.

[Lur01] J. Lurie. On simply laced Lie algebras and their minuscule representations. Comment. Math. Helv., 76(3):515-575, 2001.

[Man86] Yu. I. Manin. Cubic forms, volume 4 of North-Holland Mathematical Library. North-Holland Publishing Co., Amsterdam, second edition, 1986. Algebra, geometry, arithmetic, Translated from the Russian by M. Hazewinkel.

[MS72] D. Mumford and K. Suominen. Introduction to the theory of moduli. In Algebraic geometry, Oslo 1970 (Proc. Fifth Nordic Summer-School in Math.), pages 171-222. Wolters-Noordhoff, Groningen, 1972.

[Pey95] E. Peyre. Hauteurs et mesures de Tamagawa sur les variétés de Fano. Duke Math. J., 79(1):101-218, 1995.

[Pey98] E. Peyre. Terme principal de la fonction zêta des hauteurs et torseurs universels. Astérisque, (251):259-298, 1998. Nombre et répartition de points de hauteur bornée (Paris, 1996).

[Pey04] E. Peyre. Counting points on varieties using universal torsors. In Arithmetic of higher-dimensional algebraic varieties (Palo Alto, CA, 2002), volume 226 of Progr. Math., pages 61-81. Birkhäuser Boston, Boston, MA, 2004.

[Pop01] O. N. Popov. Del Pezzo surfaces and algebraic groups, Diplomarbeit, Universität Tübingen, 2001.

[PT01a] E. Peyre and Yu. Tschinkel. Tamagawa numbers of diagonal cubic surfaces, numerical evidence. Math. Comp., 70(233):367-387, 2001.

[PT01b] E. Peyre and Yu. Tschinkel. Tamagawa numbers of diagonal cubic surfaces of higher rank. In Rational points on algebraic varieties, volume 199 of Progr. Math., pages 275-305. Birkhäuser, Basel, 2001.

[Sal98] P. Salberger. Tamagawa measures on universal torsors and points of bounded height on Fano varieties. Astérisque, (251):91-258, 1998. Nombre et répartition de points de hauteur bornée (Paris, 1996).

[Sch63] L. Schläfli. On the distribution of surfaces of the thrid order into species, in reference to the absence or presence of singular points, and the relaity of their lines. Philos. Trans. Roy. Soc. London, 153:193-241, 1863.

[Seg42] B. Segre. The non-singular cubic surfaces. Clarendon, Oxford, 1942.

[Sko93] A. N. Skorobogatov. On a theorem of Enriques-Swinnerton-Dyer. Ann. Fac. Sci. Toulouse Math. (6), 2(3):429-440, 1993.

[Tim28] G. Timms. The nodal cubic surfaces and the surfaces from which they are derived by projection. Proc. Roy. Soc. London Ser. A, 119:213-248, 1928.

[Zie95] G. M. Ziegler. Lectures on polytopes, volume 152 of Graduate Texts in Mathematics. Springer-Verlag, New York, 1995. 



\section{Index}

algebraic group, 45

anticanonical class, 7, 9, 10, 59, 83, 109

automorphism of $\mathbb{P}^{2}, 12,22$

base point, 82

blow-up, 8, 10, $15,20,59$

canonical class, 7

Castelnuovo, 21

chamber, 13110

fundamental, 13, $18,110,113$

character, 26

circle method, 102,104

cohomology group, 25, 105

complete intersection, 47, 106

cone

affine, 45

convex, 10

effective, 10, 12, 16, 27, 59, 105, 109

114

nef, 10, 59,109

contraction, 20,83

coprimality condition, $2,119,136$

Cox ring, 2, 27, 31, 46, 57, 81

generator, $27,31,35,38,58,82,89$ 93

relation, $29,31,33,35,43,58,86,89$

cubic surface, 73

Cayley's, 88

$\mathbf{E}_{6}, 16,28,107,117$

singular, 3, 7, $16,19,19,21,93$

smooth, 1, $11,19,34,53$

curve

$(-1)-, 11,18,31,34,38,40$

$(-2)-, 16$

negative, $28,58,102$

degree, 8

Del Pezzo surface

degree 1,40

degree 2, 38,55

degree 3, see also cubic surface

degree 4 , see also quartic surface

degree 5,63

degree 6,61 generalized, $15,17,57,81,102,113$

singular, 15 102,113

smooth, 2, 8, 11, 15, 31, 45, 101, 111

toric, 29

density, 106

Dirichlet series, 129144

distinguished section, 83

divisor, 7

effective, 7, 27

exceptional, 9 , 15,134

prime, 7

divisor group, 7

Dynkin diagram, 13,15

extended, $16,$\begin{tabular}{lllllll}
18 & 34 & 59 & 89 & 94 & 136 \\
\hline
\end{tabular}

embedding, 45

anticanonical, 11, 16, 34, 59

error term, 107,118

family

embedding, 46,56

generalized, 22,96

smooth, 12

Fano variety, 101

flag variety, 45

global section, 27

grading, 27

Grassmannian, 3

halfspace, 13

height, 1, 101, 117,133

height inequality, 2, $104,124,137$

height zeta function, 103. 106

Hirzebruch surface, 16, 21, 22, 111

homogeneous coordinate ring, 27

homogeneous space, 3,47

hypersurface, 101, 103

universal torsor, 57, 118,133

integral point, 2, $103,119,136$

intersection form, 7, 18

intersection matrix, 18,59

intersection number, 7

irreducible, 29, 87

Iskovskih surface, 105 
Lebesgue measure, 109

Leray form, 106,119

Leray measure, 106

line, 1, 11, 34, 102

linear system, 82

Manin's conjecture, 2, $102,118,133$

meromorphic continuation, 107

minimal desingularization, 15

non-split, 102,133

parabolic, 45

partial ordering, 9, 27

passing through, 10

Peyre's constant, 102, 105, 109 118, 133

Picard group, 2, 7, 9,104

basis, 59

standard basis, 10

pole, 107

position

almost general, 15, 16,102

general, 11

quartic surface, 66

$\mathbf{D}_{4}, 133$

generalized, 16,19

singular, 89

smooth, 11

radical, 31,86

rational double point, 15

reflection, 13,14

representation, 46

fundamental, 45

rescaling, 46

good, 46,50

rescaling factor, 46

residue, 107, 130144

root, 13

positive, 13

simple, $13,18,45,110$

root system, 13, $18,31,45,110$

irreducible, 14,110

ruling, $31,32,39,41,47$

(2)-, 39

(3)-, 43

(n)-, 31,111

self intersection number, 7

singularity, 15, 16, 59, 110

resolution, 15, 134

split, 2, $102,109,133$

summation, 125,140

surface, 725

Tauberian theorem, 103, 107, 130, 144

toric variety, 22

torsor, 25 torus, 25

Néron-Severi, 26, 29,46

transform, 9

triangle, 34

type, 17

singularity, 1520

universal torsor, 2, 25, 26, 29, 45, 46. 57, $103,119,136$

weight, 45

weight lattice, 45

Weyl group, 13, 14, 18, 31, 45, 109 


\section{Lebenslauf}

21.07.1978 Geboren in Brakel

07/1985-06/1989 Katholische Grundschule Brakel

07/1989-06/1998 Gymnasium Brede in Brakel, abgeschlossen mit dem Abitur

07/1998-04/1999 Grundwehrdienst in Dülmen

04/1999 Beginn des Studiums der Mathematik an der Georg-August-Universität Göttingen

04/2000 Vordiplom in Mathematik

08/2001-06/2002 Auslandsstudium an der University of California, Berkeley

01/2004 Diplom in Mathematik

Betreuer der Diplomarbeit: Prof. Dr. U. Stuhler

seit 03/2004 Promotionsstudium

am Mathematischen Institut

der Georg-August-Universität Göttingen

Betreuer: Prof. Dr. Yu. Tschinkel

03/2006-05/2006 Forschungsaufenthalt am MSRI, Berkeley 